\title{
Goal setting and strategies to enhance goal pursuit for adults with acquired disability participating in rehabilitation (Review)
}

Levack WMM, Weatherall M, Hay-Smith EJC, Dean SG, McPherson K, Siegert RJ
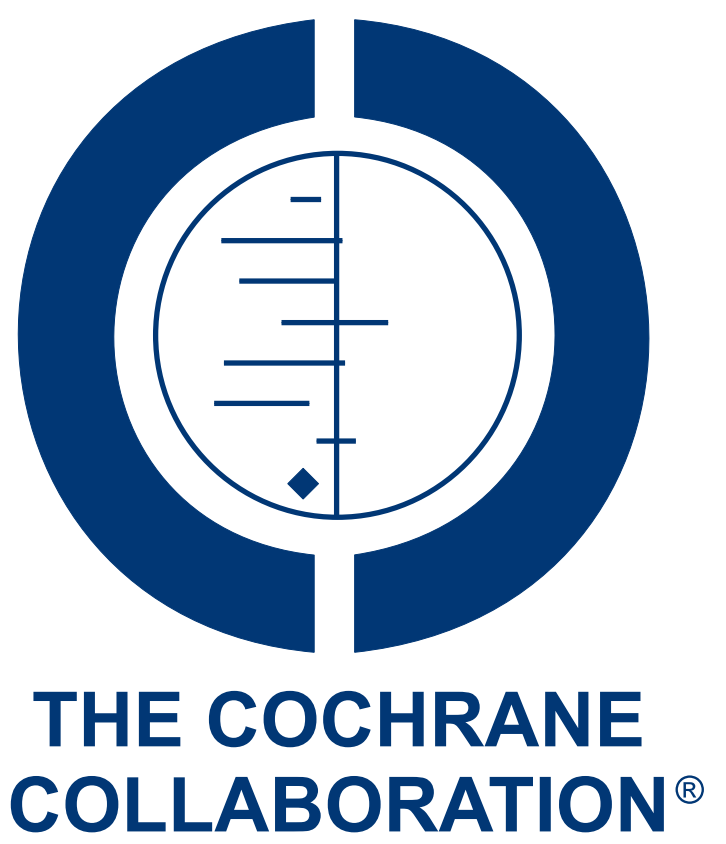

This is a reprint of a Cochrane review, prepared and maintained by The Cochrane Collaboration and published in The Cochrane Library 2015, Issue 7

http://www.thecochranelibrary.com

\section{WILEY}

Goal setting and strategies to enhance goal pursuit for adults with acquired disability participating in rehabilitation (Review) Copyright $\odot 2015$ The Cochrane Collaboration. Published by John Wiley \& Sons, Ltd. 
TABLE OF CONTENTS

HEADER . . . . . . . . . . . . . . . . . . . . . . . . . . . . . . . . . . . . . . . 1

ABSTRACT . . . . . . . . . . . . . . . . . . . . . . . . . . . . . . . . . . . . . . .

PLAIN LANGUAGE SUMMARY . . . . . . . . . . . . . . . . . . . . . . . . . . . . . . . . . . . .

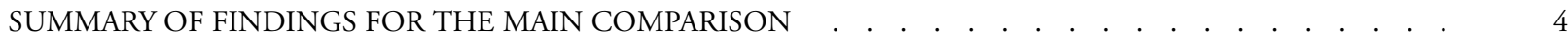

BACKGROUND . . . . . . . . . . . . . . . . . . . . . . . . . . . . . . . . . . . . . . . . . .

OBJECTIVES . . . . . . . . . . . . . . . . . . . . . . . . . . . . . . . . . . . . . . . . . . . . . . .

METHODS . . . . . . . . . . . . . . . . . . . . . . . . . . . . . . . . . . . . . . 11

RESUlTS . . . . . . . . . . . . . . . . . . . . . . . . . . . . . . . . . . . 16

Figure 1. . . . . . . . . . . . . . . . . . . . . . . . . . . . . . . . . . . . . 22

Figure 2. . . . . . . . . . . . . . . . . . . . . . . . . . . . . . . . . . . . . . . 25

Figure $3 . \quad$. . . . . . . . . . . . . . . . . . . . . . . . . . . . . . . . . . . . . . . . 26

Figure $4 . \quad$. . . . . . . . . . . . . . . . . . . . . . . . . . . . . . . . . . . . . $\quad 27$

Figure 5. . . . . . . . . . . . . . . . . . . . . . . . . . . . . . . . . . . . . . 29

Figure 6. . . . . . . . . . . . . . . . . . . . . . . . . . . . . . . . . . . . . . .

ADDITIONAL SUMMARY OF FINDINGS . . . . . . . . . . . . . . . . . . . . . . . . . . . . . . . . .

DISCUSSION . . . . . . . . . . . . . . . . . . . . . . . . . . . . . . . . . . . . . . 39

AUTHORS' CONCLUSIONS . . . . . . . . . . . . . . . . . . . . . . . . . . . . . . . . . . . . . . .

ACKNOWLEDGEMENTS . . . . . . . . . . . . . . . . . . . . . . . . . . . . . . . . . . . . . . . . 42

REFERENCES . . . . . . . . . . . . . . . . . . . . . . . . . . . . . . . . . . . . . . . . . . . . . 43

CHARACTERISTICS OF STUDIES . . . . . . . . . . . . . . . . . . . . . . . . . . . . . . . . . . . . . . . 52

DATA AND ANALYSES . . . . . . . . . . . . . . . . . . . . . . . . . . . . . . . . . . . . . . . . . . . . . . . . . .

Analysis 1.1. Comparison 1 Goal setting (with or without strategies to enhance goal pursuit) versus no goal setting,

Outcome 1 Health related quality of life or self-reported emotional status.

Analysis 1.2. Comparison 1 Goal setting (with or without strategies to enhance goal pursuit) versus no goal setting, Outcome 2 Activity - ability. . . . . . . . . . . . . . . . . . . . . . . . . . . . . . . . . .

Analysis 1.3. Comparison 1 Goal setting (with or without strategies to enhance goal pursuit) versus no goal setting, Outcome 3 Engagement in rehabilitation. . . . . . . . . . . . . . . . . . . . . . . . .

Analysis 1.4. Comparison 1 Goal setting (with or without strategies to enhance goal pursuit) versus no goal setting, Outcome 4 Self-efficacy.

Analysis 2.1. Comparison 2 Structured goal setting (with or without strategies to enhance goal pursuit) versus no structured goal setting, Outcome 1 Health related quality of life or self-reported emotional status.

Analysis 2.2. Comparison 2 Structured goal setting (with or without strategies to enhance goal pursuit) versus no structured goal setting, Outcome 2 Activity - ability.

Analysis 2.3. Comparison 2 Structured goal setting (with or without strategies to enhance goal pursuit) versus no structured goal setting, Outcome 3 Self-efficacy. . . . . . . . . . . . . . . . . . . . . . . . . . . 173

Analysis 2.4. Comparison 2 Structured goal setting (with or without strategies to enhance goal pursuit) versus no structured goal setting, Outcome 4 Satisfaction with service delivery.

Analysis 2.5. Comparison 2 Structured goal setting (with or without strategies to enhance goal pursuit) versus no structured goal setting, Outcome 5 Adverse events (withdrawal due to death, re-hospitalisation or worsening symptoms).

APPENDICES . . . . . . . . . . . . . . . . . . . . . . . . . . . . . . . . . . . . . . . . . . . 194

CONTRIBUTIONS OF AUTHORS . . . . . . . . . . . . . . . . . . . . . . . . . . . . . . . . . . . . . 198

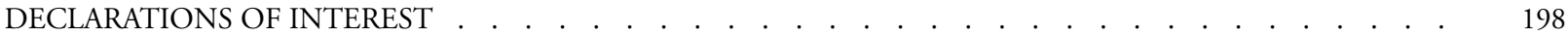

SOURCES OF SUPPORT . . . . . . . . . . . . . . . . . . . . . . . . . . . . . . . . . . . . . . . . . 199

DIFFERENCES BETWEEN PROTOCOL AND REVIEW . . . . . . . . . . . . . . . . . . . . . . . . . . . . 199

Goal setting and strategies to enhance goal pursuit for adults with acquired disability participating in rehabilitation (Review)

Copyright @ 2015 The Cochrane Collaboration. Published by John Wiley \& Sons, Ltd. 


\title{
[Intervention Review] \\ Goal setting and strategies to enhance goal pursuit for adults with acquired disability participating in rehabilitation
}

\author{
William MM Levack ${ }^{1}$, Mark Weatherall ${ }^{1}$, E. Jean C Hay-Smith ${ }^{1}$, Sarah G Dean ${ }^{2}$, Kathryn McPherson ${ }^{3}$, Richard J Siegert ${ }^{3,4}$ \\ ${ }^{1}$ Rehabilitation Teaching and Research Unit, Department of Medicine, University of Otago, Wellington, New Zealand. ${ }^{2}$ University \\ of Exeter Medical School, University of Exeter, Exeter, UK. ${ }^{3}$ School of Rehabilitation and Occupation Studies, Auckland University \\ of Technology, Auckland, New Zealand. ${ }^{4}$ School of Public Health and Psychosocial Studies, Auckland University of Technology, \\ Auckland, New Zealand \\ Contact address: William MM Levack, Rehabilitation Teaching and Research Unit, Department of Medicine, University of Otago, \\ Mein St, Newtown, PO Box 7343, Wellington, 6242, New Zealand. William.levack@otago.ac.nz. \\ Editorial group: Cochrane Consumers and Communication Group. \\ Publication status and date: New, published in Issue 7, 2015. \\ Review content assessed as up-to-date: 25 March 2014.
}

Citation: Levack WMM, Weatherall M, Hay-Smith EJC, Dean SG, McPherson K, Siegert RJ. Goal setting and strategies to enhance goal pursuit for adults with acquired disability participating in rehabilitation. Cochrane Database of Systematic Reviews 2015 , Issue 7. Art. No.: CD009727. DOI: 10.1002/14651858.CD009727.pub2.

Copyright (C) 2015 The Cochrane Collaboration. Published by John Wiley \& Sons, Ltd.

\begin{abstract}
A B S T R A C T
Background

Goal setting is considered a key component of rehabilitation for adults with acquired disability, yet there is little consensus regarding the best strategies for undertaking goal setting and in which clinical contexts. It has also been unclear what effect, if any, goal setting has on health outcomes after rehabilitation.
\end{abstract}

Objectives

To assess the effects of goal setting and strategies to enhance the pursuit of goals (i.e. how goals and progress towards goals are communicated, used, or shared) on improving health outcomes in adults with acquired disability participating in rehabilitation.

\section{Search methods}

We searched CENTRAL, MEDLINE, EMBASE, four other databases and three trials registers to December 2013, together with reference checking, citation searching and contact with study authors to identify additional studies. We did not impose any language or date restrictions.

\section{Selection criteria}

Randomised controlled trials (RCTs), cluster-RCTs and quasi-RCTs evaluating the effects of goal setting or strategies to enhance goal pursuit in the context of adult rehabilitation for acquired disability.

\section{Data collection and analysis}

Two authors independently reviewed search results for inclusion. Grey literature searches were conducted and reviewed by a single author. Two authors independently extracted data and assessed risk of bias for included studies. We contacted study authors for additional information.

Goal setting and strategies to enhance goal pursuit for adults with acquired disability participating in rehabilitation (Review)

Copyright () 2015 The Cochrane Collaboration. Published by John Wiley \& Sons, Ltd. 


\title{
Main results
}

We included 39 studies (27 RCTs, 6 cluster-RCTs, and 6 quasi-RCTs) involving 2846 participants in total. Studies ranged widely regarding clinical context and participants' primary health conditions. The most common health conditions included musculoskeletal disorders, brain injury, chronic pain, mental health conditions, and cardiovascular disease.

Eighteen studies compared goal setting, with or without strategies to enhance goal pursuit, to no goal setting. These studies provide very low quality evidence that including any type of goal setting in the practice of adult rehabilitation is better than no goal setting for health-related quality of life or self-reported emotional status ( 8 studies; 446 participants; standardised mean difference (SMD) 0.53 , $95 \%$ confidence interval (CI) 0.17 to 0.88 , indicative of a moderate effect size) and self-efficacy (3 studies; 108 participants; SMD $1.07,95 \%$ CI 0.64 to 1.49 , indicative of a moderate to large effect size). The evidence is inconclusive regarding whether goal setting results in improvements in social participation or activity levels, body structure or function, or levels of patient engagement in the rehabilitation process. Insufficient data are available to determine whether or not goal setting is associated with more or fewer adverse events compared to no goal setting.

Fourteen studies compared structured goal setting approaches, with or without strategies to enhance goal pursuit, to 'usual care' that may have involved some goal setting but where no structured approach was followed. These studies provide very low quality evidence that more structured goal setting results in higher patient self-efficacy ( 2 studies; 134 participants; SMD 0.37 , $95 \%$ CI 0.02 to 0.71 , indicative of a small effect size) and low quality evidence for greater satisfaction with service delivery ( 5 studies; 309 participants; SMD $0.33,95 \%$ CI 0.10 to 0.56 , indicative of a small effect size). The evidence was inconclusive regarding whether more structured goal setting approaches result in higher health-related quality of life or self-reported emotional status, social participation, activity levels, or improvements in body structure or function. Three studies in this group reported on adverse events (death, re-hospitalisation, or worsening symptoms), but insufficient data are available to determine whether structured goal setting is associated with more or fewer adverse events than usual care.

A moderate degree of heterogeneity was observed in outcomes across all studies, but an insufficient number of studies was available to permit subgroup analysis to explore the reasons for this heterogeneity. The review also considers studies which investigate the effects of different approaches to enhancing goal pursuit, and studies which investigate different structured goal setting approaches. It also reports on secondary outcomes including goal attainment and healthcare utilisation.

\section{Authors' conclusions}

There is some very low quality evidence that goal setting may improve some outcomes for adults receiving rehabilitation for acquired disability. The best of this evidence appears to favour positive effects for psychosocial outcomes (i.e. health-related quality of life, emotional status, and self-efficacy) rather than physical ones. Due to study limitations, there is considerable uncertainty regarding these effects however, and further research is highly likely to change reported estimates of effect.

\section{PLAIN LANGUAGESUMMARY}

\author{
Goal setting for adults receiving clinical rehabilitation for disability
}

\section{Background}

Goal setting is considered a key part of clinical rehabilitation for adults with disability, such as in rehabilitation following brain injuries, heart or lung diseases, mental health illnesses, or for injuries or illnesses involving bones and muscles. Health professionals use goals to provide targets for themselves and their clients to work towards. In this review we summarise studies that have investigated what effect, if any, goal setting activities have on achieving good health outcomes following rehabilitation.

\section{Results}

This review found 39 studies published before December 2013, involving a total of 2846 participants receiving rehabilitation in a variety of countries and clinical situations. The studies used a wide range of different approaches to goal setting and tested the effectiveness of these approaches in a number of different ways. Overall these studies provide very low quality evidence that goal setting helps patients achieve a higher quality of life or sense of well-being and a higher belief in their own ability to achieve goals that they choose to pursue. There is currently no consistent evidence that goal setting improves people's functional abilities after rehabilitation or how hard they try with therapeutic interventions during rehabilitation.

Goal setting and strategies to enhance goal pursuit for adults with acquired disability participating in rehabilitation (Review) 
Insufficient information exists to say whether goal setting increases or reduces the risk of adverse events (such as death or re-hospitalisation) for people involved in rehabilitation. Because of the variety of approaches to studying goal setting in rehabilitation and because of limitations in the design of many studies completed to date, it is very possible that future studies could change the conclusions of this review. We also need more research to improve our understanding of how components of the goal setting process (such as how difficult goals are, how goals of therapy should be selected and prioritised, how goals are used in clinical practice, and how feedback on progress towards goals should be provided) contribute or do not contribute to better health outcomes. 


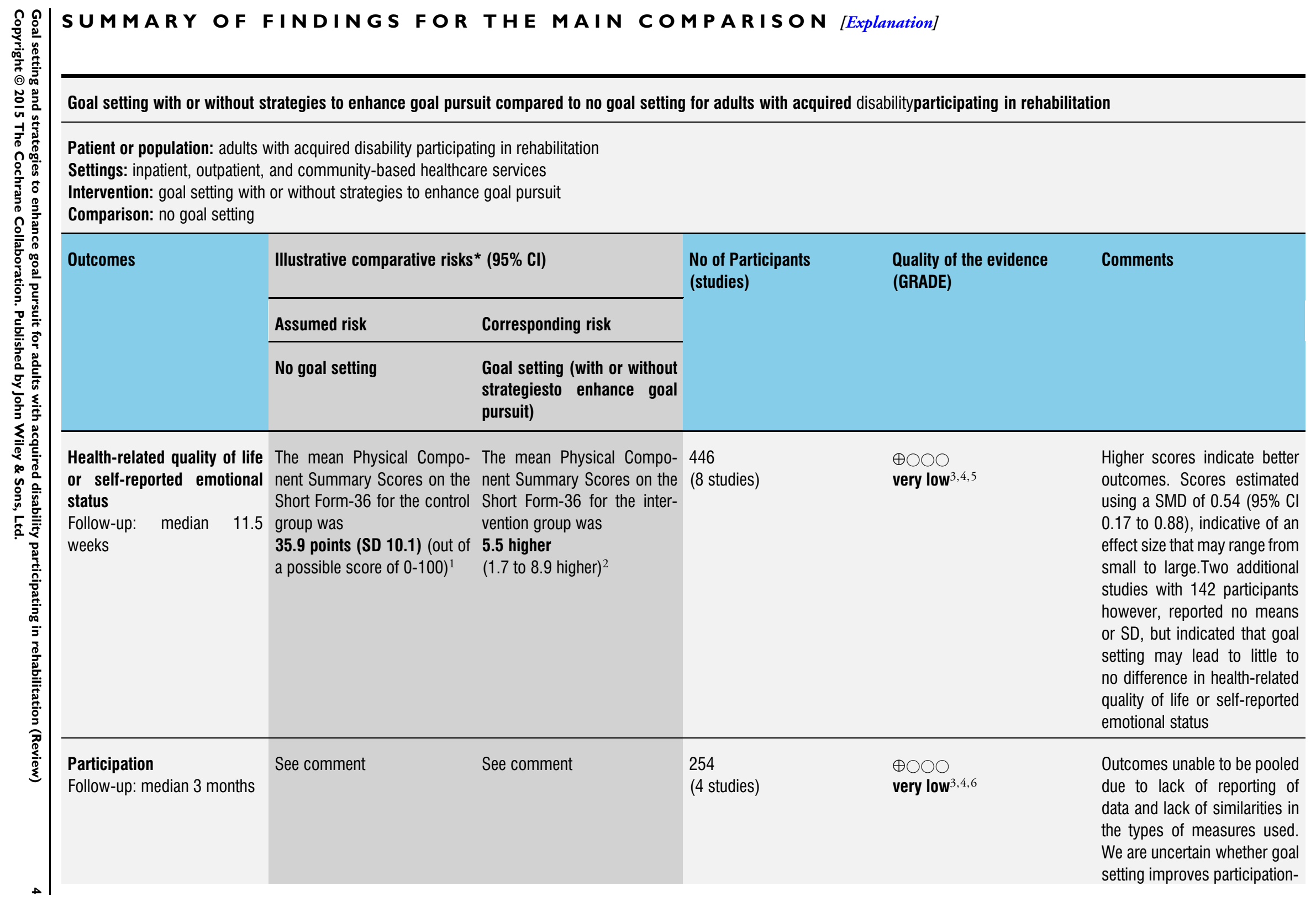




\begin{tabular}{|c|c|c|c|c|c|}
\hline & & & & & level outcomes \\
\hline $\begin{array}{l}\text { Activity } \\
\text { Follow-up: median } 18 \text { weeks }\end{array}$ & $\begin{array}{l}\text { The mean Barthel Index score } \\
\text { for the control group was } \\
\mathbf{1 8} \text { points (SD 3.3) (out of a } \\
\text { possible score of } 0-20)^{7}\end{array}$ & $\begin{array}{l}\text { The mean Barthel Index score } \\
\text { for the intervention groups } \\
\text { was } \\
0.1 \text { higher } \\
(0.7 \text { lower to } 1 \text { higher })^{2}\end{array}$ & $\begin{array}{l}223 \\
\text { (4 studies) }\end{array}$ & $\begin{array}{l}\oplus \oplus \bigcirc \bigcirc \\
\text { low }^{3,6}\end{array}$ & $\begin{array}{l}\text { Higher scores indicate better } \\
\text { outcomes. Scores estimated } \\
\text { using a SMD of } 0.04 \text { ( } 95 \% \mathrm{CI} \\
-0.22 \text { to } 0.31 \text { ). This evidence } \\
\text { suggests that goal setting may } \\
\text { not improve activity-level out- } \\
\text { comes }\end{array}$ \\
\hline
\end{tabular}

$\begin{array}{lll}\text { Body structure and body See comment } & \text { See comment } & 235\end{array}$

Follow-up: median 3 months

$\begin{array}{ll}\text { (5 studies) } & \oplus \bigcirc \bigcirc \bigcirc \\ \text { very low } & \end{array}$

to lack of similarities in the types of measures used. We are uncertain whether goal setting improves outcomes at the level of body structure and body function

Engagement in rehabilitation The mean number of hours The intervention groups 369

(motivation, involvement and worked on a 26-week support worked

(9 studies)

$\oplus \bigcirc \bigcirc$

adherence)

work placement programme $\mathbf{5 0}$ hours more

very $10 w^{4,6,8,11}$

Follow-up: median 8.5 weeks for the control groups was (12 hour less to 110 hours 255 hours of work (SD 166) more) $)^{2}$ on a 26 -week support 10

work placement programme

Higher scores indicate bet-

ter engagement. Scores estimated using a SMD of 0 . $30(95 \% \mathrm{Cl}-0.07$ to 0.66$)$ One additional study with 27 participants reported no means or SD but indicated that goal setting may lead to little to no difference in engagement in rehabilitation. One further study with 367 participants measured medication regime adherence as a dichotomous variable, and reported that the odds for the goal setting group adhering was 1.13 times higher $(95 \% \mathrm{Cl}$ 1.08 to 1.19 ) than that of the 


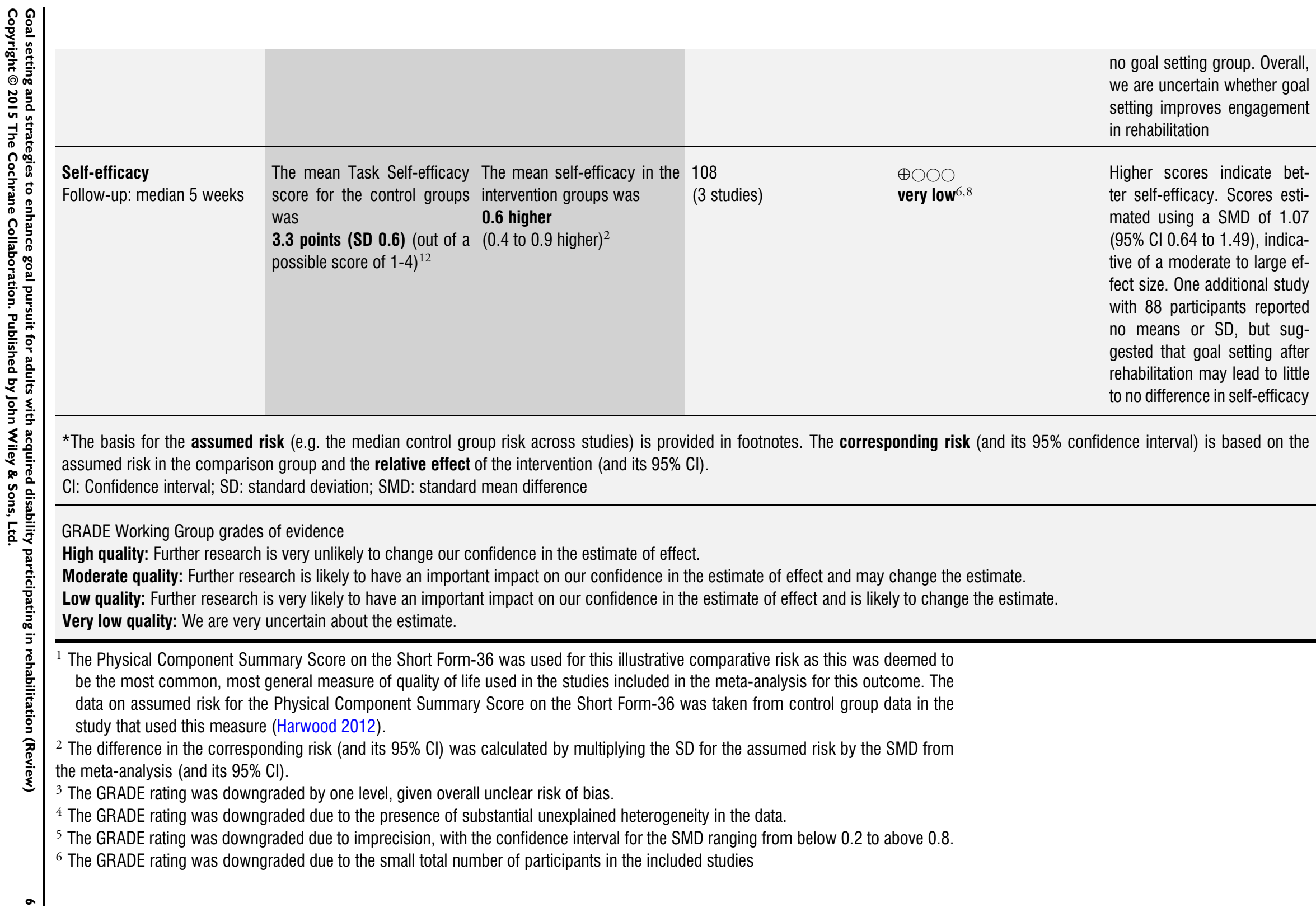




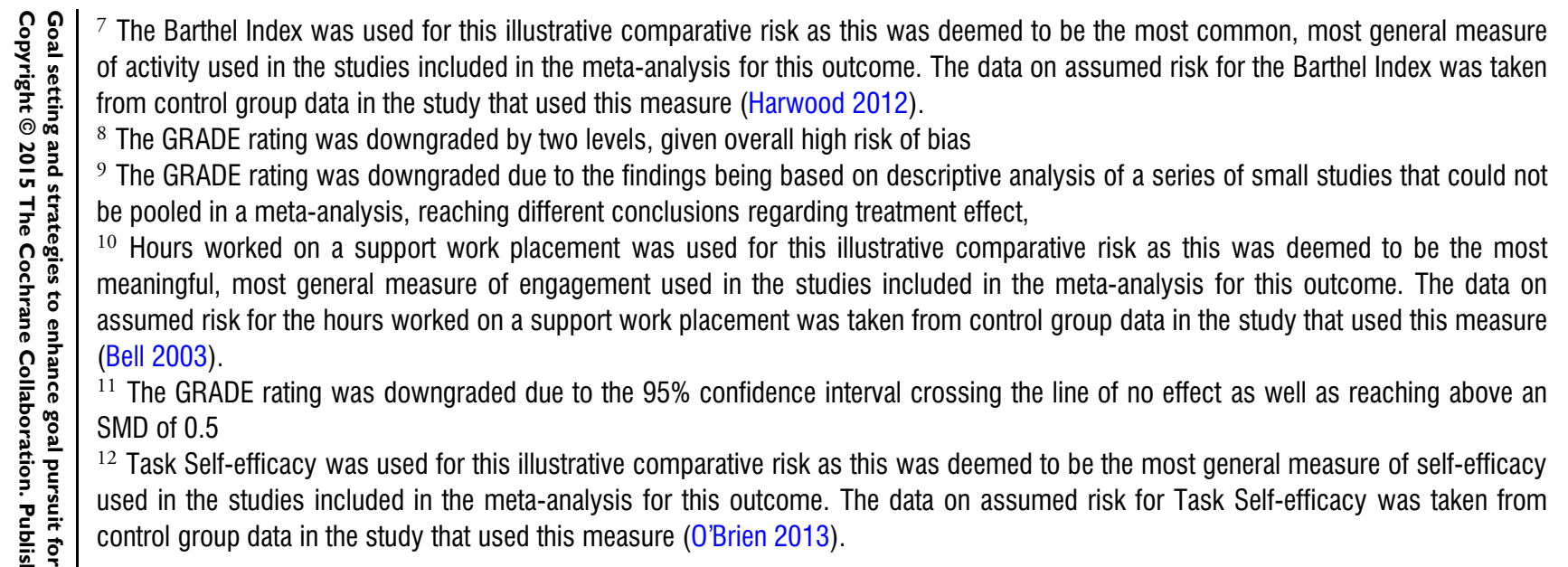




\section{B A C K G R O U N D}

Goal setting is considered an essential part of clinical rehabilitation. It has been described as a core practice within rehabilitation (Wade 2009), a requirement for effective interdisciplinary teamwork (Schut 1994), and an activity that specifically characterises both rehabilitation services and those who provide them (Barnes 2000; Scobbie 2009; Wade 1998). In clinical practice there has been growing emphasis on the need for interventions with patients to be goal oriented. Goal terminology is becoming integral to discussions of guidelines, policies and professional requirements at both regional and international levels (e.g. Duncan 2005; Evans 2001; Randall 2000; RCP 2003; RCP 2004; Rothstein 2003).

Some authors have suggested that evidence for the effectiveness of goal setting in improving patient outcomes has already been firmly established, and that this evidence can now direct how goal setting in rehabilitation should be implemented (Black 2010; Marsland 2010; Wilson 2008). However, a systematic review of randomised controlled trials (RCTs) concluded that the evidence regarding any generalisable effect of goal setting on patient outcomes following rehabilitation was inconsistent at best, and greatly limited by the quality of studies published at the time (Levack 2006a). Given that this review is now over nine years old, there is a need to update this work.

\section{Description of the condition}

This review focuses on the application of goal setting in the context of rehabilitation for adults with acquired disability. The term 'disability' is defined according to the World Health Organization's (WHO) International Classification of Functioning, Disability and Health (ICF) as an 'umbrella term for impairments, activity limitations or participation restrictions' (WHO 2001a, p.3) that result from interactions between a person (with a health condition) and that person's contextual factors (environmental factors and personal factors). For the purposes of this review, the term 'acquired disability' is used to refer more specifically to disability that arises during a person's adult life (i.e. after 16 years of age) following an accident, illness or development of a health condition. This term therefore excludes disability associated with health conditions arising prenatally or in childhood.

\section{Description of the intervention}

Reviews of literature on goal setting in rehabilitation are complicated by a number of factors, one of which is the difficulty that exists in describing what might (or might not) constitute 'goal setting' in a rehabilitation context. The terms 'goals', 'goal setting' and 'goal planning' have been used to refer to many different constructs with little current consensus around key terminology (Levack 2006b; Playford 2009). A range of different approaches to goal setting has been described in the literature, with various similarities and differences in the recommended process and content of each. These include (but are not limited to):

- Goal Attainment Scaling (GAS) (Kiresuk 1968; Turner-Stokes 2009);

- goal setting based on the Canadian Occupational Performance Measure (COPM) (Pendleton 2005; Phipps 2007; Trombly 2002; Wressle 2002; Wressle 2003);

- 'SMART' goal planning (Barnes 2000; Bovend'Eerdt 2009; Mastos 2007; McLellan 1997; Monaghan 2005; Schut 1994);

- 'RUMBA' goal planning (Barnett 1999);

- Self-Identified Goal Assessment (Melville 2002);

- Goal Management Training (Levine 2000);

- approaches to goal planning from the Wolfson

Neurorehabilitation Centre (McMillan 1999)

- contractually-organised goal setting (Powell 2002);

- Collaborative Goal Technology (Clarke 2006);

- goal setting as part of the Progressive Goal Attainment Programme (Sullivan 2006);

- patient-centred functional goal planning (Randall 2000); and

- goal setting based on the Patient Goal Priority Questionnaire or Patient Goal Priority List (Asenlöf 2009).

Note: 'SMART' and 'RUMBA' are not abbreviations, but mnemonic acronyms for key components of goal setting, promoted by various authors. Interpretations of these acronyms differ (McPherson 2014; Wade 2009). One interpretation of the 'SMART' acronym is that it stands for Specific, Measurable, Achievable, Relevant, and Time-limited goals (Barnes 2000). Similarly, it is suggested that 'RUMBA' refers to Relevant, Understandable, Measurable, Behavioural, and Achievable goals (Barnett 1999).

While these different approaches to goal setting frequently include common features, such as having measurable goals, or patient involvement in goal selection, few such features are universal to all recommended approaches. Indeed, all approaches to goal setting in rehabilitation differ from one another across a number of variables, including:

- the group intended to use the approach (i.e. for use by a single, specific profession or for use by an interprofessional team);

- the intended patient population for the approach;

- the process by which goals are selected (e.g. who is involved; how goals are identified and prioritised);

- the recommended characteristics of the actual goals set (i.e. how goals are written; whether they need to be phrased in a certain way);

- the recommended content of goals set (i.e. what is considered an acceptable topic for a goal; whether goals need to be set at a particular level of the ICF);

- the way the goals are subsequently used in clinical environments (e.g. the way goals are used in team meetings or meetings with patients; how feedback on progress towards goals 
is presented and used in clinical interactions); and

- the intended purpose(s) of setting and having goals.

Even for individually-named approaches to goal setting, opinions can differ in terms of how each approach should be implemented. For instance, multiple variations on the original GAS approach (Kiresuk 1968) exist, such as: involving greater patient participation in goal selection (Cytrynbaum 1979; LaFerriere 1978; Malec 1999; Turner-Stokes 2009); having the treating therapist rather than an independent third party select and re-evaluate the GAS goals (Cytrynbaum 1979; Turner-Stokes 2009; Willer 1976); using a different number of 'levels' of goal achievement and a different scoring system than was originally proposed (LaFerriere 1978; Turner-Stokes 2010; Willer 1976), or using standardised rather than individualised wording to indicate the extent of goal achievement (Turner-Stokes 2009). Similarly, there is no one agreed 'SMART' approach to goal setting; the 'SMART' acronym has been interpreted to refer to a range of goal-related concepts, and there is no consensus regarding the 'correct' interpretation of this approach (McPherson 2014; Wade 2009).

Goal setting is also often presented as a core component of a whole programme of intervention (e.g. Stuifbergen 2003). However, a systematic review of research into the effectiveness of goal setting needs to be able to separate out the independent effects of goal setting from those of other variables associated with these programmes of intervention (e.g. the amount of therapeutic activity, amount of additional education and information, or other behavioural interventions that are not related to the setting of rehabilitation goals). For more information on the history of goal setting and its application in rehabilitation please refer to Levack 2014a.

\section{Definition of 'rehabilitation goal'}

Within the field of psychology there is an enormous body of literature describing and analysing goal constructs from many perspectives. In this context, the term 'goals' has been defined as 'internal representations of desired states, where states are broadly construed as outcomes, events, or processes' (Austin 1996, p.338). This definition allows for goals that are consciously set as well as goals which are not; goals for individuals as well as goals for whole organisations or populations of people; biological goals (such as to change one's body temperature or reproduce); complex cognitive or aesthetic goals (such as to live a moral life or achieve a career objective); goals that relate to a moment in time and goals that relate to a lifespan. From this perspective, all human behaviour is goal directed.

In the context of rehabilitation however, the term 'goal' is generally used to mean something much more specific, and more explicitly linked to clinical work. For the purpose of this review we use the term 'rehabilitation goal' to refer to the concept of a 'goal' set for the purposes of clinical work in rehabilitation, in order to make a clear distinction between this type of goal and colloquial use of the term 'goal' or broader definitions of 'goals' from psychology. One proposed definition of the term 'rehabilitation goal' has been 'a future state that is desired and/or expected. The state might refer to relative changes or to an absolute achievement. It might refer to matters affecting the patient, the patient's environment, the family or any other party. It is a generic term with no implications about time frame or level' (Wade 1998, p.273). Other authors, focusing on describing an approach to goal setting intended for a particular patient population or for use by one professional group, have been more specific in their definition of goals for rehabilitation. For example, Randall 2000 defined a 'functional goal' within the context of physical therapy as 'the individually meaningful activities that a person cannot perform as a result of an injury, illness, or congenital or acquired condition, but wants to be able to accomplish as a result of physical therapy' (p.1198).

In contrast, many 'goals' in the psychological sense of the word are implicit (i.e. goals which are implied without being directly stated or even necessarily consciously set). For example, the act of reaching for a cup is a motor activity with an implicit goal. Asking a patient to reach for a cup versus reaching into mid-air is an example of using implicit goals to influence behaviour (Trombly 1999). However, using such activities as a clinical intervention (e.g. for exercise therapy after a stroke) is not an example of 'goal setting' in rehabilitation in its usual sense. While (as stated above) all human behaviour is arguably goal directed and rehabilitation cannot therefore occur without having 'goals' of some kind, it is not true that all goals are 'rehabilitation goals' in the sense usually intended by rehabilitation teams.

Furthermore, the concept of a 'rehabilitation goal' usually refers to a relationship between an individual patient and an individual or group of health professionals (and/or others). This excludes goals set at an organisational level (e.g. in the case of health service management) or community level (e.g. in the case of public health policy) from the definition of 'goal setting' in a rehabilitation context. In other words, while goals such as 'to reduce the incidence of falls in hospital' may be an important key performance indicator for a particular rehabilitation service, these types of organisational goals are not what is usually being discussed in the literature on goal setting in rehabilitation.

Therefore, for the purpose of this review, we define 'rehabilitation goal' as: a desired future state to be achieved by a person with a disability as a result of rehabilitation activities. Rehabilitation goals are actively selected, intentionally created, have purpose and are shared (wherever possible) by the people participating in the activities and interventions designed to address the consequences of acquired disability.

\section{Definition of 'goal setting'}

From a literal perspective, the term 'goal setting' refers solely to the selection of goals. For the purposes of this review, we define 'goal setting' more broadly as: the establishment or negotiation 
of rehabilitation goals. Consistent with other clinical researchers publishing in this area (Wade 1998), we will consider 'goal setting' to be synonymous with 'goal planning'.

\section{Definition of 'goal pursuit'}

In addition to the establishment or negotiation of rehabilitation goals, there are a number of activities related to how rehabilitation goals are communicated, used or shared that are intended to enhance how effective or successful people are in working towards those goals. For the purposes of this review we will use the term 'goal pursuit' to refer to these additional goal-related activities. These activities include: development of a plan or strategy to achieve stated rehabilitation goals, provision of explicit feedback (oral or written) on a person's progress towards their rehabilitation goals, and use of strategies to maintain or enhance commitment to set goals (such as peer discussion of progress toward an individual's rehabilitation goals, or use of posters and electronic diaries reminding people about their rehabilitation goals). As the behavioural effects of having a goal are often moderated by a number of factors (e.g. people's ability to develop a plan to reach their goal, their awareness of how their current abilities or performance compares with that required to achieve their goal, and their level of commitment to specific goals) it is important not to exclude these factors from a systematic review of the therapeutic effects of goal setting in rehabilitation contexts.

\section{How the intervention might work}

Goal setting has been attributed with multiple purposes (or functions). Levack $2006 \mathrm{~b}$ presents a brief typology of purposes from the clinical literature, and Levack 2006c provides an overview of purposes attributed to goal setting by health professionals working in rehabilitation environments for people with acquired brain injury. These papers highlight a number of reasons why rehabilitation professionals might believe goal setting is important in clinical practice.

- Goal setting might improve patient outcomes, by:

$\circ$ improving the patient's motivation to engage in therapeutic activities;

○ improving clinical teamwork (providing teams with shared direction; focusing collaborative interprofessional practice);

o enhancing the working relationship between patients, families and health professional (e.g. through development of a shared language and shared understanding of a health condition and the rehabilitation process);

$\circ$ improving the patient's ability to self-regulate desirable behaviour (e.g. by retraining self-awareness or addressing goal neglect in patients with problems in those areas);

$\circ$ assisting patients (and their family) to adapt psychologically to the consequences of disability; or $\circ$ enhancing specificity of training (e.g. focusing therapy for an individual on performance of a specific activity in a specific environment relevant to that individual's daily life).

- Goal setting might enhance patient self-determination (i.e. autonomy) - considered by some to be an important reason to undertake goal setting regardless of other outcomes achieved or not achieved in terms of health and functioning.

- The degree of goal attainment might be a useful measure of health outcome.

- Goal setting is a contractual or legislative requirement of service delivery.

While clinicians, patients or family members may have different opinions about the main reason for undertaking goal setting in rehabilitation, for this review the improvement of patient outcomes is of greatest interest. One important point here is that goal setting as an intervention for improving health outcomes for patients should be considered separately from goal setting for the purpose of outcome evaluation (where 'outcomes' are evaluated in terms of 'goal achievement'). In other words, goal setting as an intervention (i.e. as a way of engaging with people with acquired disability) may be effective in terms of achieving higher levels of improvement in a person's functional abilities (to pick just one type of outcome) without the specific goals of rehabilitation for that person necessarily being reached.

In terms of how goal setting might influence patient motivation or self-regulation in clinical environments in order to achieve improvements in patient outcomes, a number of additional theories from psychology have been suggested as relevant to rehabilitation, including:

- Bandura's Social Cognitive Theory;

- Locke and Latham's Goal Setting Theory;

- Schwarzer's Health Action Process Approach;

- Aspin and Taylor's Proactive Coping Theory;

- Leventhal's Self-Regulation Model of Illness Behaviour; and

- Carver and Scheiers' Control-Process Model of Self-

Regulation.

An overview of these theories and their application to rehabilitation has been given elsewhere (Scobbie 2009; Siegert 2004; Siegert 2014a). Broadly speaking, these theories describe: how people use and respond to goals in order to monitor, alter or adapt their behaviour; how emotional responses to goals or progress toward goals influence future goal-oriented behaviour; how perceptions of illness and perceptions of the effect of interventions influence goaloriented behaviour; and how the effects of goals can be moderated by various factors such as personal goal commitment, beliefs in one's ability to achieve a goal (self-efficacy), task complexity, and the way goals are presented or worded.

In clinical practice, several other variables may influence the success of goal setting interventions. These include how meaningful the goals are to the individual patient (how committed patients are to the goals; how well they relate to their higher-order life pri- 
orities) and how involved patients are in the selection of goals, factors that might reasonably be considered to contribute to the 'person-centredness' of the goal-setting approach (Wilson 2008). Also, how 'reasonable' or 'realistic' a goal is to achieve (Wilson 2008) and how involved family members and significant others are in the selection of goals have been considered important (Levack 2009; McMillan 1999; Visser-Meily 2006; Wade 1999a). Lastly, some researchers and clinicians have proposed that goal setting is likely to be more successful when goals are set at the level of 'activity' and 'participation' than when goals are established to address impairments at the level of body structure and body function (Marsland 2010; Randall 2000). For example, a goal to be able to transfer independently from a wheelchair to a toilet (an activitylevel goal) or to return to paid employment (a participation-level goal) would be considered, in general, more effective for improving patient outcomes than a goal to improve muscle strength of the quadriceps by 150\% (a goal set at the level of body structure and body function).

\section{O B J E C T I V E S}

To assess the effects of goal setting, and strategies to enhance goal pursuit, on health outcomes in adults with acquired disability participating in rehabilitation. To test the following comparisons:

1. a structured approach to goal setting, with or without strategies to enhance goal pursuit versus no goal setting;

2. a structured approach to goal setting, with or without strategies to enhance goal pursuit versus 'usual care' that may involve some goal setting but where no structured approach was followed;

3. interventions to enhance goal pursuit versus no interventions to enhance goal pursuit; and

4. one structured approach to goal setting and/or strategies to enhance goal pursuit versus another structured approach to goal setting and/or strategies to enhance goal pursuit.

\section{METHODS}

\section{Why it is important to do this review}

There is extensive research from education (Boekaerts 2000; Pintrich 2000), industrial-organisational psychology (Latham 2007; Locke 2002), cognitive psychology (Austin 1996; Custers 2010; Moskowitz 2009) and sport psychology (Burton 2010; Hall 2001; Wilson 2006) which has demonstrated the effect that goals can have on human behaviour. It seems reasonable to assume that goal setting could have a similar type of effect in populations of people participating in clinical rehabilitation. However, what this broad body of research has also demonstrated is that the effectiveness of goal setting and the mechanism by which goals achieve these effects can be highly dependent on context. For instance, it has been found that theories of goal setting from industrial-organisational psychology are not as effective when applied to goal setting in the context of sport psychology, leading to the development of new theories of goal setting specific to the sport environment (Hall 2001).

As highlighted above, multiple approaches to goal setting in rehabilitation exist and several different mechanisms are suggested by which goals might affect patient outcomes. Furthermore, there is debate about the evidence for the effectiveness of goal setting for improving patient outcomes, and the most recent systematic review of this literature is now several years old (Levack 2006a). There is a need for a Cochrane review regarding the effects of goal setting or strategies to enhance goal pursuit to influence patient outcomes in rehabilitation for adults with acquired disability. This review is beneficial for determining whether the evidence shows that goal setting or strategies to enhance goal pursuit are effective interventions, as well as providing possible directions for future research into the use of rehabilitation goals in clinical work.

\section{Criteria for considering studies for this review}

\section{Types of studies}

Randomised controlled trials (RCTs), cluster-RCTs, or quasiRCTs (where allocation to study groups was by a method that was not truly random, such as alternation, assignment based on date of birth, case record number or date of presentation, or due to use of stratification or minimisation).

\section{Types of participants}

People receiving rehabilitation for disability acquired in adulthood (e.g. after 16 years of age).

For the purposes of this review 'disability' was defined according to the ICF as an 'umbrella term for impairments, activity limitations or participation restrictions' (WHO 2001a, p.3) that result from interactions between a person (with a health condition) and that person's contextual factors (environmental factors and personal factors). Thus, we excluded studies investigating the application of goal setting to health interventions for non-disabled people (e.g. in public health or obstetric contexts). More specifically, this review included people with disability arising from injuries, illnesses or disorders, as categorised by the WHO (WHO 1992), involving:

- the musculoskeletal system or connective tissue;

- the skin or subcutaneous tissue;

- the cardiac system (including the cerebrovascular system);

- the respiratory system;

- the nervous system; 
- the sensory system (e.g. eye, ear etc);

- the endocrine, nutritional or metabolic system;

- the genitourinary system; and

- mental or behavioural function.

For the purposes of this review 'rehabilitation' was defined as 'a process aimed at enabling persons with disabilities to reach and maintain their optimum physical, sensory, intellectual, psychiatric and/or social functional levels, thus providing them with the tools to change their lives towards a higher level of independence. The rehabilitation process does not, however, involve initial medical care' (WHO 2001b, p.290). Thus, we excluded studies investigating the effects of goal-directed decision-making by medical staff in emergency or intensive care settings, or in the management of acute medical conditions such as sepsis.

\section{Types of interventions}

We included studies that investigated the effects of establishing and negotiating rehabilitation goals, with or without strategies to enhance goal pursuit. For the purposes of this review, the term 'rehabilitation goals' refers to an actively selected and desired future state to be achieved by a person with a disability as a result of rehabilitation activities.

We included studies that investigated:

- a structured approach to goal setting with or without strategies to enhance goal pursuit in comparison to no goal setting; or

- a structured approach to goal setting with or without strategies to enhance goal pursuit in comparison to 'usual care' that may involve some goal setting but where no structured approach was followed; or

- interventions to enhance goal pursuit in comparison to no interventions to enhance goal pursuit; or

- one structured approach to goal setting and/or strategies to enhance goal pursuit in comparison to another structured approach to goal setting and/or strategies to enhance goal pursuit.

For the purposes of this review, approaches to goal setting were considered to differ if they involved different methods for:

- identification, negotiation, or selection of rehabilitation goals; or

- documentation of rehabilitation goals; or

- involvement of health professionals, patients, family members or other significant people in the selection of rehabilitation goals.

Approaches to enhancing goal pursuit were considered to differ if they involve different methods for:

- developing a plan on how to attain rehabilitation goals;

- providing feedback to patients on their performance towards rehabilitation goals; or

- enhancing patient commitment to attain rehabilitation goals.
We excluded studies investigating approaches to goal setting as an intervention compared to some other intervention intended to influence human cognition or behaviour (e.g. priming for pain attention in the case of Stenstrom 1994). We excluded any study that did not adequately control for additional treatment variables separate to the goal setting intervention. Hence we excluded studies in which goal setting formed only part of a whole programme of rehabilitation, where the outcomes of the intervention could not be specifically attributed to goal setting or to components of the goal setting process (e.g. Glasgow 2000).

\section{Types of outcome measures}

We excluded studies investigating only the immediate effects of goal setting. Studies were categorised as investigating the immediate effects of goal setting if they involved implementation of goal setting and collection of data on the effects of goal setting (e.g. in terms of immediate improvements in effort or performance on a set task) during only one session for each study participant, carried out over the course of less than one day (e.g. Gauggel 2001).

We prioritised the following outcomes.

\section{Primary outcomes}

- Health-related quality of life or self-reported emotional status.

- Participation outcomes as defined by the ICF (WHO 2001a), e.g. work, community integration, social relationships.

- Activity outcomes as defined by the ICF (WHO 2001a), e.g. activities of daily living, mobility.

\section{Secondary outcomes}

- Outcomes at the level of body structure and function as defined by the ICF (WHO 2001a).

- Patient self-belief and engagement in rehabilitation, e.g. adherence, patient motivation, self-efficacy.

- Individual goal attainment.

- Evaluation of care, e.g. satisfaction with care.

- Service delivery level, e.g. cost of care, length of stay.

- Adverse outcomes, e.g. complications, morbidity, mortality, readmission rate.

N.B. Individual goal attainment was not included as a primary outcome measure in this review as achievement of individualised goals is in part based on changes in health status achieved by rehabilitation patients, and in part based on the level of difficulty of the individually-selected goals. It is possible therefore for two people to achieve the same degree of functional recovery (or gain in other outcomes) following rehabilitation, but score differently on measures based on achievement of individualised goals. As there is scope for debating what such differences in individualised goal attainment mean, we chose to select individual goal attainment as a secondary outcome.

Goal setting and strategies to enhance goal pursuit for adults with acquired disability participating in rehabilitation (Review) 


\section{Search methods for identification of studies}

\section{Electronic searches}

We searched the following electronic databases in September 2012, with an updated search conducted in January 2014 for articles published to the end of December 2013.

- The Cochrane Central Register of Controlled Trials (CENTRAL; 2013, Issue 12).

- MEDLINE (OvidSP) (1950 to December 2013).

- EMBASE (OvidSP) (1988 to December 2013).

- PsycINFO (OvidSP) (1967 to December 2013).

- CINAHL (EBSCOhost) (1981 to December 2013).

- AMED (OvidSP) (1985 to December 2013).

- Proquest Dissertations and Theses database (1673 to

December 2013).

Detailed search strategies are presented in Appendices 1 to 7. We did not impose any language restrictions.

We searched the ProQuest Dissertations and Theses database for grey literature. We also searched databases in the WHO Clinical Trial Search Portal (www.who.int/trialsearch), Australian New Zealand Clinical Trials Registry (http://www.anzctr.org.au/), and Current Controlled Trials (www.controlled-trials.com) to identify ongoing or recently completed studies (Appendix 8).

\section{Searching other resources}

We contacted experts in the field and authors of included studies for advice as to other relevant studies. We also searched reference lists of relevant studies and personal collections of articles. We sought full research reports of any potentially eligible studies that were published as abstracts or conference proceedings only. We included studies only published as abstracts or conference proceedings in the review where sufficient information about the study methods and data could be extracted from the abstract, published proceedings, or poster presentation, supplemented by author communication, with all sources of information noted in the section in Included studies. Where sufficient information could not be uncovered on potentially eligible studies, we excluded these studies, giving lack of information as the reason for this in the section in Excluded studies.

\section{Data collection and analysis}

The data collection and analysis methods were described in the review protocol (Levack 2012).

\section{Selection of studies}

Two review authors (WL and RS) independently screened all search results (titles and abstracts) for possible inclusion, and those selected by either or both authors were subject to full-text assessment. The two review authors then independently assessed the selected articles for inclusion. The same two review authors resolved differences in the first instance by discussion, and then by input from a third review author from the review team. The whole review team debated particularly difficult decisions regarding inclusion. Any studies thus excluded but considered near the boundary for possible inclusion have been reported in Characteristics of excluded studies with the reason for exclusion given. We also present relevant ongoing studies in Characteristics of ongoing studies.

\section{Data extraction and management}

We used a standard data extraction form adapted from the Cochrane Consumers and Communication Review Group's Data Extraction Template for all included studies. Two review authors (WL and RS) independently assessed the risk of bias in included studies and independently extracted data for each study. The two review authors resolved differences in the first instance by discussion, and then by input from a third author from the review team. Review authors were not blinded to the names of study authors, journals or institutions.

For included studies, we extracted data on the intervention aims, study aims, study design, methods used, characteristics of participants (e.g. age, gender, ethnicity, socioeconomic status, principal health condition, inclusion of people with multimorbidities), characteristics of the study setting (e.g. geographic location, specific clinical context, co-interventions being provided alongside goal setting), characteristics of the approach(es) to goal setting and strategies to enhance goal pursuit under investigation, outcome measures used, and reported findings.

Data extracted to categorise the approach to goal setting or goal pursuit under investigation included (if specified in the study's method):

- the name of the approach to goal setting (e.g. GAS, SMART, COPM);

- the health professional(s) involved in goal setting;

- the training of health professionals for their involvement in goal setting;

- the level of patient and/or family involvement in goal selection (e.g. whether goals were: prescribed with no input from study participants; selected through discussion and negotiation with the patient; selected through discussion and negotiation with the patient and their family; or selected by the study participants with no involvement of other parties);

- the type of communication used for making selected goals explicit (e.g. written, oral);

- whether or not the intervention involved an explicit process for developing a plan to achieve the stated goal(s), and if so what this was;

- whether or not goals for study participants were made public to others (e.g. other patients); 
- whether or not study participants were reminded about their goals during the course of rehabilitation;

- whether or not study participants were provided with feedback on their progress towards goals during the course of rehabilitation;

- whether or not there was the development of written 'contracts' with participants to pursue specified goals;

- assessment of the participants' level of commitment to attain their goals;

- the level of goal difficulty (and how this was specified or quantified by the researchers); and

- the targeted level of functioning for specific goals (e.g. if goals were set at the level of body structure and body function, activity, or participation, as defined by the ICF, WHO 2001a).

The first author (WL) then entered the data into Review Manager (RevMan 2014), with another author (RS) checking the accuracy of data entry.

\section{Assessment of risk of bias in included studies}

We assessed and reported on the risk of bias of included studies in accordance with the guidelines of the Cochrane Consumers and Communication Review Group (Ryan 2011), which recommends the explicit reporting of the following individual 'Risk of bias' elements for RCTs: random sequence generation; allocation concealment; blinding (participants, personnel), blinding (outcomes assessment); incomplete outcome data (attrition bias; adequacy of intention-to-treat analysis); selective outcome reporting; other sources of bias (e.g. recruitment bias, baseline imbalance, loss of clusters, incorrect analysis, and comparability with RCT based on per person randomisation in the case of cluster-RCTs; and suitability of cross-over design, management of carry-over effect, incorrect analysis, comparability of results with parallel-group trials, treatment period effects, and randomisation of order of treatments in the case of cross-over design RCTs).

We conducted 'Risk of bias' assessments in accordance with the Cochrane Handbook for Systematic Reviews of Interventions (Higgins 2011), with risk of bias being rated as high risk, unclear risk or low risk for each element and for each study overall. We used the same criteria for assessment of risk of bias for quasi-RCTs; these studies were rated as being at high risk of bias both for random sequence generation and allocation concealment.

In all cases, two authors (WL and RS) independently assessed the risk of bias in included studies. These two review authors resolved differences in the first instance by discussion, and then by input from a third author from the review team. We attempted to contact study authors for additional information about the included studies or for clarification of the study methods as required. In the case of studies where one of the study authors was also an author of this review (i.e. McPherson 2009 and Taylor 2012), another review author took the lead on the 'Risk of bias' assessment.

\section{Measures of treatment effect}

Three categories of primary outcomes were the focus of this review: 1. health-related quality of life or self-reported emotional status;

2. participation outcomes; and

3. activity outcomes, as defined by the ICF (WHO 2001a).

We adopted the approach used by Brennan 2009 and Horvat 2014 for selection and extraction of primary outcomes from included studies. We included any primary outcome identified by study authors that fell within the scope of the primary outcomes categories listed above. If multiple primary outcomes were identified within any category, we ranked the reported effect estimates for each of these outcomes and selected the outcome with the median effect estimate. If no primary outcome within our categories was specified, we adopted the following strategy. First we used any outcome within our categories specified in sample size calculations; then, if necessary, we ranked relevant intervention effect estimates, as reported, and selected the median effect estimate. If the number of outcomes was even (n), we included the outcome whose effect estimate was ranked $n / 2$. We have reported in our results whether we used the primary outcome or the outcome with the median effect estimate. Where possible, we also verified whether the specified primary outcomes in included studies were consistent with those identified in trial protocols, trial registry entries or both. We extracted the intervention effect estimate reported for all included outcomes (both primary and secondary outcomes) with the associated $\mathrm{P}$ value and $95 \%$ confidence intervals (CIs), and the method of statistical analyses used to calculate them. For continuous data, where outcomes were measured in a standard way across studies, we reported the mean difference (MD) and 95\% CI. Where outcomes were measured using different scales (e.g. for quality of life) we calculated a standardised mean difference (SMD) and 95\% CI. When calculating a SMD within each outcome category, we multiplied all mean values for reversed scored outcomes (where lower scores indicate a better outcome) by -1 to ensure that the direction of all scales (from better to worse outcomes) were consistent.

For dichotomous data, where outcomes were measured in a standard way we reported the risk ratio (RR) and 95\% CI. For categorical outcomes (such as employment outcomes) we related the numbers reporting an outcome to the numbers at risk in each group to derive a RR. We dichotomised ordinal data (such as Likert scales for symptom improvement) and managed them as a categorical outcome. We treated GAS scores as ordinal rather than interval data, as recommended by Steenbeek 2007 and Tennant 2007 , and we treated count data as continuous data.

\section{Unit of analysis issues}

The primary analysis was planned on the basis of per person randomisation. For all studies we considered the possibility of unit of analysis issues arising from the inclusion of cluster-randomised 
designs, repeated measurements and studies with more than two treatment groups. When applicable, we dealt with unit of analysis issues by analysing the data according to recommendations in the Cochrane Handbook for Systematic Reviews of Interventions (Higgins 2011). In cluster-RCTs, we first sought to use effect estimates and standard errors that were adjusted for clustering, combining the studies using the generic inverse-variance method. When analysis in a cluster-RCT did not take account of clustering, then we attempted to approximate the cluster-adjusted effect size and standard error based on available data if the unadjusted effect estimate, the number or size of clusters, and the intraclass correlations were provided. If the intraclass correlation coefficient could not be obtained then we endeavoured to use an estimate from similar studies. If none of these options were possible, we included the studies unadjusted for clustered in the analyses, but then tested the effect doing this by examining the results of analyses with these studies removed. In studies with repeat observations (collecting data using the same measures on participants at a number of different time points) we selected the longest follow-up data from each study. If studies had more than two groups we combined all relevant experimental intervention groups of the study into a single group, and combined all relevant control intervention groups into a single control group.

\section{Dealing with missing data}

If data were missing from the relevant comparisons we attempted to contact the study authors to obtain the information. Where studies did not state that results were reported using an intentionto-treat analysis for primary outcomes, we contacted the study authors to request data to enable us to conduct such an analysis. If no response from authors was provided, we analysed results as reported.

\section{Assessment of heterogeneity}

Given the potential for clinical and methodological diversity in studies that might have been eligible for inclusion, it was important to consider heterogeneity in the data analysis. Clinical heterogeneity was determined before analysis of data by extracting and considering information on each study's patient populations, clinical contexts, approaches to goal setting, and outcome measures used.

We identified statistical heterogeneity in studies thought to be clinically and methodologically similar by visual inspection of forest plots and by using a standard $\mathrm{Chi}^{2}$ test and a significance level of alpha $=0.1$, in view of the low power of such tests. We also examined heterogeneity with $\mathrm{I}^{2}$, where $\mathrm{I}^{2}$ values of $50 \%$ or more were deemed to indicate a substantial level of heterogeneity (Higgins 2003). We used a random-effects model to assess heterogeneity as, prior to conducting the review, we had anticipated finding substantive differences in the patient populations, rehabilitation settings and approaches to the selection and use of goals in the included studies.

\section{Assessment of reporting biases}

We assessed the extent of publication bias through visual inspection of asymmetry and running the regression-based method for a funnel plot in Review Manager 5 (RevMan 2014). We considered other forms of reporting bias (e.g. multiple publication bias, location bias, language bias, outcome reporting bias) on review of the full papers for each included study. The possibility of reporting bias is presented in the results below.

\section{Data synthesis}

We began the data synthesis with a narrative overview of the findings in the form of a table. The review authors, as a team, considered the comparability of the participants, clinical contexts, approaches to goal setting, and types of outcome data in order to determine whether statistical pooling of results was appropriate. Where appropriate, we used meta-analytical methods to pool outcome data from sufficiently homogeneous studies to calculate effects in the comparisons outlined in our Objectives. Following data extraction, but prior to data analysis, we made the post-hoc decision to combine measures of self-reported emotional status with self-reported measures of health-related quality of life. We did this because few studies reported measures of health-related quality of life and because the two concepts were deemed to be sufficiently similar for the results of a meta-analysis to be clinically meaningful: quality of life, for instance, often has an emotional health subscale. We conducted all analyses according to guidelines in the Cochrane Handbook for Systematic Reviews of Interventions (Higgins 2011). We assessed quality of evidence using GRADE, and have presented a summary of the results of the data synthesis and assessment of the quality of the evidence in a 'Summary of findings' table. In 'Summary of findings for the main comparison' and in 'Summary of findings 2' we included summary information on the following: health-related quality of life or self-reported emotional status, participation outcomes, activity outcomes, outcomes at the level of body structure and function, patient engagement in rehabilitation, and self-efficacy.

\section{Subgroup analysis and investigation of heterogeneity}

Where there were sufficient data (i.e. at least 10 studies) and where it was appropriate in the context of the study, we planned to conduct subgroup analysis on the basis of four factors:

- level of patient and/or family involvement in goal selection;

- level on the ICF at which rehabilitation goals were set;

- level of goal difficulty; and

- presence of cognitive or psychiatric impairments in study populations. 
However, we did not identify sufficient studies to permit any of these subgroup analyses.

\section{Sensitivity analysis}

We undertook sensitivity analyses to examine the influence of risk of bias associated with including the studies in each meta-analysis. We removed studies at the greatest risk of bias (i.e. those that failed to randomise adequately or failed to conceal random allocation) from the analysis in order to test the strength of evidence for the various effect estimates.

\section{Consumer participation}

We invited consumer referees to comment on the protocol and on the completed review through standard Cochrane Consumers and Communication Review Group editorial processes.

\section{RE S U L T S}

\section{Description of studies}

\section{Results of the search}

We ran searches in September 2012, and again in January 2014, generating 9019 records, after removing duplicates. We screened the titles and abstracts of all citations and identified 151 articles that were potentially eligible for inclusion. We reviewed these in full text against the selection criteria, and identified 39 studies that met the inclusion criteria. Seven of these 39 studies were reported in multiple publications (see Table 1).

\section{Included studies}

Thirty-nine studies met the selection criteria for this review (see Characteristics of included studies).

\section{Comparison groups}

\section{Comparison 1}

Of the 39 trials, 18 compared a structured approach to goal setting, with or without strategies to enhance goal pursuit, to no goal setting (Bassett 1999; Bell 2003; Blair 1991; Blair 1996; Coote 2012; Coppack 2012; Cross 1971; Duncan 2003; Evans 2002; Fredenburgh 1993; Harwood 2012; Howell 1986; Iacovino 1997; Mann 1987; O’Brien 2013; Scott 2004; Sewell 2005; Stanhope 2013).

\section{Comparison 2}

Fourteen trials compared a structured approach to goal setting, with or without strategies to enhance goal pursuit, to 'usual care' that may have involved goal setting but where no structured approach to goal setting was followed (Arnetz 2004; Asenlof 2005; Cheng 2012; Gagné 2003; Hart 1978; Holliday 2007; Jonsdottir 2012; LaFerriere 1978; McPherson 2009; Oestergaard 2012; Ostelo 2003; Parsons 2012; Taylor 2012; Woltmann 2011). Four of the studies in this comparison group were described by the authors as being pilot studies or feasibility studies (Gagné 2003; Jonsdottir 2012; McPherson 2009; Taylor 2012).

\section{Comparison 3}

Two trials investigated an intervention where the only difference to a control group was the use of a strategy to enhance goal pursuit (Culley 2010; Hart 2002).

\section{Comparison 4}

Nine trials compared one structured approach to goal setting and/or strategies to enhance goal pursuit to another structured approach to goal setting and/or strategies to enhance goal pursuit (Bassett 1999; Blair 1991; Blair 1996; Conrad 2000; James 1993; McPherson 2009; Miller 2012; Richardson 2007; Webb 1994). Four of these nine trials had more than two goal setting intervention groups, permitting their inclusion in more than one comparison in this review (Bassett 1999; Blair 1991; Blair 1996; McPherson 2009). For studies that had more than one intervention group, we only included the groups that met the inclusion criteria for analysis.

\section{Communication with study authors}

Of the 39 included studies, we found that 5 were reported in sufficient detail such that we required no further information about these studies for the purpose of this review (Hart 2002; Harwood 2012; James 1993; Ostelo 2003; Taylor 2012). We attempted to contact the authors of each of the other 34 studies to obtain additional information, and were successful with 19 (Arnetz 2004; Asenlof 2005; Bassett 1999; Bell 2003; Coote 2012; Conrad 2000; Culley 2010; Duncan 2003; Evans 2002; Holliday 2007; Jonsdottir 2012; LaFerriere 1978; McPherson 2009; Miller 2012; O’Brien 2013 Parsons 2012; Scott 2004; Sewell 2005; Stanhope 2013), although full details were only available for 15 of these studies (Asenlof 2005; Bassett 1999; Coote 2012; Conrad 2000; Culley 2010; Duncan 2003; Evans 2002; Jonsdottir 2012; LaFerriere 1978; McPherson 2009; Miller 2012; O’Brien 2013; Parsons 2012; Sewell 2005; Stanhope 2013). We had at least one unanswered question about methods or outcome data for 24/39 $(62 \%)$ of the included studies. 


\section{Types of studies}

\section{Unit of randomisation}

Six studies used a cluster-RCT design, randomising groups of participants clustered on the basis of the residential facility in which they lived (Blair 1991), the family physician with whom they were registered (Parsons 2012), the healthcare organisation or hospital providing their services (Cheng 2012; Stanhope 2013; Taylor 2012), or on the basis of the case manager who was managing their rehabilitation planning (Woltmann 2011). For three of these studies (Parsons 2012; Stanhope 2013; Taylor 2012) the effects of clustering on means and SDs in the outcome measures reported could be estimated from the information provided in the paper or from additional information provided by the study authors. For the other three studies (Blair 1991; Cheng 2012; Woltmann 2011) information on the effects of clusters on the data reported could not be accessed or estimated from other related publications. For these three studies we chose to include the data as reported in the published paper, but to report in our results where this occurred, and to test the influence of these decisions in our sensitivity analyses. For two other studies, randomisation occurred at the level of the participants' goals rather than at the level of the participant, with each participant having multiple goals being randomly allocated to either an intervention condition for enhancing goal recall or a control condition (Culley 2010; Hart 2002). For these two studies raw outcome data were available from the published paper or via author communication, so our analysis could be conducted at the same level as the unit of randomisation.

\section{Sample sizes}

Sample sizes ranged from 7 to 367 , with a total of 2846 participants in the 39 studies addressing the 4 main comparisons in the review. Six of the included studies were reported as being pilot studies or feasibility studies (Gagné 2003; Jonsdottir 2012; McPherson 2009; Miller 2012; Richardson 2007; Taylor 2012) and therefore were not designed to have a sufficiently large sample size to detect statistically significant differences.

\section{Types of settings}

Seven of the included studies were conducted within inpatient hospital settings. This included three studies conducted within multidisciplinary units for people with neurological conditions (Holliday 2007; Jonsdottir 2012; Taylor 2012), one study conducted within a rheumatology unit (Arnetz 2004), two studies conducted within orthopaedic surgical units (Cross 1971; Oestergaard 2012), and one study conducted in a number of different hospital ward settings (Gagné 2003). An eighth study was conducted in both residential and outpatient-based rehabilitation services for people with brain injury (Culley 2010). Eleven additional studies were conducted in outpatient or primary care set- tings, including four studies conducted within a cardiovascular rehabilitation service (Conrad 2000; Duncan 2003; Iacovino 1997; Mann 1987), one study conducted within a pulmonary rehabilitation service (Sewell 2005), one study conducted within an exercise laboratory (O'Brien 2013), four studies conducted within physiotherapy services (Asenlof 2005; Bassett 1999; Evans 2002; Ostelo 2003), and one study conducted within a cognitive behavioural therapy programme for chronic pain (James 1993). One additional study was undertaken in a short-term residential rehabilitation unit for chronic pain (Coppack 2012).

The remaining 19 included studies were conducted in community and/or residential care settings. These included eight studies conducted within community-based mental health services (Bell 2003; Coote 2012; Fredenburgh 1993; Hart 1978; Howell 1986; LaFerriere 1978; Stanhope 2013; Woltmann 2011), two studies conducted within residential care services for older adults (Blair 1991; Blair 1996), two studies conducted within community-based diabetes services (Miller 2012; Richardson 2007), three studies conducted as part of home-based nursing services (Cheng 2012; Parsons 2012; Scott 2004), three studies conducted within residential and home-based service for people with brain injury (Hart 2002; McPherson 2009; Webb 1994), and one study conducted in people's homes after stroke (Harwood 2012).

Of the 39 included studies, 17 were conducted in the United States of America (USA) (Bell 2003; Blair 1991; Blair 1996; Cross 1971; Duncan 2003; Fredenburgh 1993; Gagné 2003; Hart 1978; Hart 2002; James 1993; LaFerriere 1978; Miller 2012; Richardson 2007; Scott 2004; Stanhope 2013; Webb 1994; Woltmann 2011), 7 in the United Kingdom (UK) (Coote 2012; Coppack 2012; Culley 2010; Evans 2002; Holliday 2007; Howell 1986; Sewell 2005), 6 in New Zealand (Bassett 1999; Harwood 2012; McPherson 2009; O’Brien 2013; Parsons 2012; Taylor 2012), 3 studies in Canada (Conrad 2000; Iacovino 1997; Mann 1987), 2 in Sweden (Arnetz 2004; Asenlof 2005), and 1 in each of Denmark (Oestergaard 2012), Switzerland (Jonsdottir 2012), Hong Kong (Cheng 2012), and the Netherlands (Ostelo 2003).

\section{Types of participants}

Participants (people receiving services) in the 39 included studies were adults receiving rehabilitation interventions for neurological conditions, including stroke in eight studies (Culley 2010; Hart 2002; Harwood 2012; Holliday 2007; Jonsdottir 2012; McPherson 2009; Taylor 2012; Webb 1994), musculoskeletal or chronic pain conditions in 10 studies (Arnetz 2004; Asenlof 2005; Bassett 1999; Coppack 2012; Cross 1971; Evans 2002; James 1993; O’Brien 2013; Oestergaard 2012; Ostelo 2003), mental health conditions in 8 studies (Bell 2003; Coote 2012; Fredenburgh 1993; Hart 1978; Howell 1986; LaFerriere 1978; Stanhope 2013; Woltmann 2011), cardiovascular conditions in 5 studies (Conrad 2000; Duncan 2003; Iacovino 1997; Mann 1987; Scott 2004), age-related disability in 3 studies (Blair 1991; Blair 1996; Parsons 2012), diabetes in 2 studies (Miller 2012; 
Richardson 2007), and respiratory disorders in 1 study (Sewell 2005). The remaining 2 studies involved a mixed sample of patients with chronic disabling conditions (Cheng 2012; Gagné 2003).

\section{Types of interventions}

A range of different approaches to goal setting were employed in the included studies. Fifteen studies involved one or more named approaches to goal setting. Of these, seven employed GAS or a modified version of GAS as the method of goal setting under investigation (Arnetz 2004; Blair 1991; Blair 1996; Hart 1978; Howell 1986; LaFerriere 1978; Webb 1994). Three other studies investigated the effect of the COPM as a method of person-centred goal setting (Oestergaard 2012; Sewell 2005; Taylor 2012). Five further studies investigated the effect of goal setting and strategies to enhance goal pursuit based on use of the 'ICF Rehab Cycle' (Jonsdottir 2012), the Patient Goal Priority Questionnaire (Asenlof 2005), the TARGET method of goal setting (Parsons 2012), Goal Management Training and Identity Oriented Goal Training (McPherson 2009), and the Goal Setting and Planning skills (GAP) programme (Coote 2012).

Twenty-four other studies did not investigate the use of a specifically named approach to goal setting, but several of these did refer to theories of goal setting when describing the approach that was employed. Three of these studies used an approach to goal setting based on King's nursing theory of goal attainment (Cheng 2012; Fredenburgh 1993; Scott 2004). Eight other studies referred to Locke and Latham's goal theory and/or Bandura's Social Cognitive Theory when the background to goal setting in rehabilitation was described (Cross 1971; Culley 2010; Duncan 2003; Evans 2002; Holliday 2007; Iacovino 1997; Mann 1987; Miller 2012). However, of the studies that referred to Locke and Latham's goal theory, only two applied goal setting in a manner consistent with Locke and Latham's model, investigating the effect of difficult or challenging goals on human performance (Iacovino 1997; Miller 2012). One study investigated the effect of goal setting based on the theory of operant conditioning and its application via contingency management (Ostelo 2003), and another used Personal Construct Theory to guide goal setting in a rehabilitation context (Coppack 2012). Yet another study based strategies for enhancing goal pursuit on a theory of behavioural change called intentions implementation (O’Brien 2013). The remaining ten studies, did not employ a named approach to goal setting, or refer to any specific goal theory when the approach to goal setting under investigation was described (Bassett 1999; Bell 2003; Conrad 2000; Gagné 2003; Hart 2002; Harwood 2012; James 1993; Richardson 2007; Stanhope 2013; Woltmann 2011).

\section{Involvement of participants in goal setting}

Of the 39 included studies, 31 employed an approach to goal setting where the participants receiving services were actively in- volved in the selection of goals for therapy (Arnetz 2004; Asenlof 2005; Bassett 1999; Bell 2003; Blair 1991; Blair 1996; Cheng 2012; Coote 2012; Coppack 2012; Evans 2002; Fredenburgh 1993; Gagné 2003; Hart 1978; Harwood 2012; Holliday 2007; Howell 1986; Iacovino 1997; Jonsdottir 2012; LaFerriere 1978; Mann 1987; McPherson 2009; O’Brien 2013; Oestergaard 2012; Ostelo 2003; Parsons 2012; Scott 2004; Sewell 2005; Stanhope 2013; Taylor 2012; Webb 1994; Woltmann 2011). For most of these studies, selection of goals was described as a collaborative activity with therapists working as guides or coaches to assist the people receiving services set relevant goals for intervention. One of these studies compared this approach to goal setting to another approach where goals were prescribed by the treating therapist and to a third (control) group for whom therapy was provided without any goals being set (Bassett 1999). However, in three studies, while participants were involved in the selection of goals for therapy, their choice of goals was restricted to those from a prespecified list of goals (Mann 1987; Blair 1991; Blair 1996). Conversely, in two other studies, participants receiving the intervention had a much higher level of control over the selection of goals, with the researchers only providing them with training in goal setting, and ultimately leaving the application of this training up to the individuals themselves (i.e. self-directed rehabilitation) (Coote 2012; Harwood 2012).

In six included studies, participants were not involved in goal selection at all (Conrad 2000; Cross 1971; Duncan 2003; James 1993; Miller 2012; Richardson 2007). Among these studies, four involved goals that were prescribed on the basis of group allocation, where the type of goal was manipulated as the independent variable in the study (Conrad 2000; James 1993; Miller 2012; Richardson 2007). The other two studies involved goals that were prescribed by the clinician providing the intervention (Duncan 2003), or where the same goal was allocated to all participants in the intervention arm of the study (Cross 1971). Two further studies did not report on who had been involved in goal selection or how the goals had been chosen (Culley 2010; Hart 2002).

\section{Involvement of family members in goal setting}

Only two studies reported family members as being actively involved in goal setting (Holliday 2007; Harwood 2012). In one of these studies, the involvement of family members was part of a wider aim to address cultural dimensions of the delivery of the intervention, with the researchers also ensuring that all interventions were delivered by a person of the same ethnicity as those receiving the interventions (Harwood 2012). In the other study, the involvement of family members in goal setting was encouraged but not required (Holliday 2007). In a third study, a 'collateral' person was involved in the goal setting process; however in this case, the relationship between this 'collateral' person and the person receiving care was not reported, and their role was solely to provide 'external validation of the patient's self-report' (Hart 1978, p. 1244). A fourth study did not include family members 
in the goal setting process, but did involve them in the application of strategies to pursue goal achievement (Conrad 2000). The remaining 34 included studies did not report on the involvement of family members in goal setting or strategies to enhance goal pursuit.

\section{Individual versus group-based goal discussion}

In one study, all goal selection and goal monitoring occurred within a small-group context with peers who were also participants in the study (Bell 2003). For this study, all individual goals and goal achievements during the study were disclosed to these peers. In another study, a similar approach to group-based goal discussion and goal monitoring was undertaken, but the group sizes ranged from one to four individuals, meaning that the trial involved some group-based and some individual approaches to goal discussions (Iacovino 1997). In one other study, a familycentred approach was taken to goal setting, meaning that again, all individual goals were shared with other people in a close relationship to the person receiving services (Harwood 2012). In a fourth study, all goal setting occurred on an individual basis, but all subsequence discussion of goals occurred within the context of group-based rehabilitation classes (Sewell 2005). The other 34 included studies reported only on goal discussions occurring at an individual level with each study participant.

\section{Goal difficulty}

Six of the included studies emphasised the setting of realistic, achievable goals (Bassett 1999; Blair 1991; Cheng 2012; Duncan 2003; Holliday 2007; O’Brien 2013). One study emphasised the setting of challenging, difficult goals (Iacovino 1997). Two other studies investigated the effect of setting difficult, challenging goals versus less difficult goals (Conrad 2000; Miller 2012). One study involved therapists evaluating the level of difficulty of goals, but where goal difficulty was not apparently restricted by the goal setting process involved (Webb 1994). The remaining 30 studies did not report on whether any strategies were used to direct, monitor, or limit the level of difficulty of goals that were set.

\section{Area or topic of focus for goals set}

Two of the included studies focused on setting goals related to impairments of body structure and body function or on activity limitations (Arnetz 2004; Evans 2002). Nine studies focused on setting goals solely to do with addressing activity limitations (Bassett 1999; Blair 1991; Blair 1996; Duncan 2003; Gagné 2003; Oestergaard 2012; Ostelo 2003; Richardson 2007; Sewell 2005). Two other studies involved setting goals either to do with addressing activity limitations or participation restrictions (Holliday 2007; Taylor 2012). One study just referred to a focus on 'functional' goals (O'Brien 2013). Two studies focused entirely on goals related to work performance (Bell 2003; Iacovino 1997). Four studies involved goals about dietary behaviour (Conrad 2000; Cross 1971; Mann 1987; Miller 2012). One study only involved goals regarding the amount and frequency of practice of specific coping strategies for managing pain (James 1993). Four studies included a mix of topics as the focus of goal setting covering a wide area of future objectives such as functional abilities, emotional needs, information needs, financial needs, and so on (Cheng 2012; Coppack 2012; Harwood 2012; McPherson 2009). The topic of focus for goals was not specifically reported on in the 15 other included studies in this review (Asenlof 2005; Coote 2012; Culley 2010; Fredenburgh 1993; Hart 1978; Hart 2002; Howell 1986; Jonsdottir 2012; LaFerriere 1978; McPherson 2009; Parsons 2012; Scott 2004; Stanhope 2013; Webb 1994; Woltmann 2011).

\section{Documentation of goals for participants}

In 15 studies, written copies of goals were kept by the participants receiving the rehabilitation intervention (Asenlof 2005; Bassett 1999; Bell 2003; Conrad 2000; Coote 2012; Duncan 2003; Gagné 2003; Harwood 2012; Iacovino 1997; James 1993; McPherson 2009; O'Brien 2013; Ostelo 2003; Richardson 2007; Webb 1994). For four of these studies, the participants themselves were responsible for documenting their own goals (Bell 2003; Coote 2012; Harwood 2012; Iacovino 1997). In two studies all goals were recorded on a goal setting form designed for the study, but it was not clear whether this form was held by the patient or the clinician involved in delivery of the intervention (Cheng 2012; Evans 2002). For two other studies, the method of documentation of goals was the subject of the trial, so differed for the intervention and control goals (Culley 2010; Hart 2002). It was not reported in the remaining 20 studies whether participants were given a written copy of their goals (Arnetz 2004; Blair 1991; Blair 1996; Coppack 2012; Cross 1971; Fredenburgh 1993; Hart 1978; Holliday 2007; Howell 1986; Jonsdottir 2012; LaFerriere 1978; Mann 1987; Miller 2012; Oestergaard 2012; Parsons 2012; Scott 2004; Sewell 2005; Stanhope 2013; Taylor 2012; Woltmann 2011).

\section{Evaluation of goal commitment}

The level of commitment of participants to achieve goals that had been set was evaluated in only two studies (Iacovino 1997; Miller 2012). In one study the behavioural intentions of participants towards goal achievement were assessed using Likert-type questions (Iacovino 1997). In the other study a questionnaire was used to evaluate the participants' level of determination to achieve goals at two time points: once following goal assignment and once at the end of the study (Miller 2012). The other 37 included studies did not report on the evaluation of goal commitment. 


\section{Development of a plan for goal pursuit}

Twenty-eight of the included studies described structured processes for the development of a plan to pursue goal achievement (Asenlof 2005; Bell 2003; Blair 1991; Blair 1996; Cheng 2012; Conrad 2000; Coote 2012; Cross 1971; Duncan 2003; Fredenburgh 1993; Hart 1978; Harwood 2012; Holliday 2007; Howell 1986; Iacovino 1997; James 1993; Jonsdottir 2012; Mann 1987; McPherson 2009; Miller 2012; O’Brien 2013; Oestergaard 2012; Ostelo 2003; Parsons 2012; Richardson 2007; Scott 2004; Sewell 2005; Stanhope 2013). Development of a plan for goal achievement was not relevant to two other studies because the aim of these studies was just to investigate strategies to enhance recall of goal over a period of time (Culley 2010; Hart 2002). The remaining nine studies did not report on strategies used to develop a plan for goal achievement (Arnetz 2004; Bassett 1999; Coppack 2012; Evans 2002; Gagné 2003; LaFerriere 1978; Taylor 2012; Webb 1994; Woltmann 2011).

\section{Reminders for participants about goals}

Twenty-four of the included studies reported on the use of a strategy to assist participants (receiving services) to remember that they had rehabilitation goals and what these goals were (Asenlof 2005; Bassett 1999; Bell 2003; Cheng 2012; Conrad 2000; Coote 2012; Coppack 2012; Culley 2010; Duncan 2003; Evans 2002; Gagné 2003; Hart 1978; Hart 2002; Howell 1986; Iacovino 1997; James 1993; Mann 1987; McPherson 2009; Miller 2012; Ostelo 2003; Richardson 2007; Sewell 2005; Stanhope 2013; Webb 1994). For 15 of these studies, discussion of goals or reminders about goals featured as part of regular (usually weekly) therapy sessions (Asenlof 2005; Bassett 1999; Bell 2003; Cheng 2012; Coppack 2012; Evans 2002; Hart 1978; Howell 1986; Iacovino 1997; James 1993; Mann 1987; McPherson 2009; Ostelo 2003; Sewell 2005; Webb 1994). In 5 other studies, reminders about goals were provided less frequently: every 3 weeks over the study period (Duncan 2003), monthly for 11 months (Stanhope 2013), at 2 and 4 month meetings during a 7-month study period (Conrad 2000), and just once midway through the study intervention (Coote 2012; Miller 2012). One study used a goal notebook to remind participants about their rehabilitation goals (Gagné 2003), and three further studies used electronic methods including text messaging (Culley 2010), portable voice organisers (Hart 2002), and the Internet (Richardson 2007). The remaining 16 studies included in this review did not report on the use of strategies to remind participants about their goals during the course of the intervention.

\section{Active monitoring of progress towards goals}

Sixteen of the included studies reported on the use of strategies to monitor progress towards achievement of goals and discussion of this information with study participants (Asenlof 2005; Bassett 1999; Bell 2003; Cheng 2012; Coppack 2012; Duncan 2003; Evans 2002; Hart 1978; Iacovino 1997; Mann 1987; McPherson
2009; Miller 2012; Ostelo 2003; Richardson 2007; Stanhope 2013; Webb 1994). Progress towards goal achievement was selfrated by participants (receiving services) in the case of five studies (Iacovino 1997; Mann 1987; Miller 2012; Ostelo 2003; Webb 1994), with four of these studies employing self-monitoring forms or charts to facilitate this process (Mann 1987; Miller 2012; Ostelo 2003; Webb 1994), and one study using an online Internet service with data on exercise performance uploaded directly from pedometers to facilitate participant self-monitoring of progress towards goals (Richardson 2007). Progress towards goals was discussed with therapists at regular meetings (usually weekly) in the case of 11 studies (Asenlof 2005; Bassett 1999; Bell 2003; Cheng 2012; Coppack 2012; Evans 2002; Hart 1978; Iacovino 1997; Mann 1987; McPherson 2009; Ostelo 2003), with goals being revised as participants progressed in the case of two studies (Bassett 1999; Mann 1987). In one study, progress towards goals was discussed with therapists at just one point midway through the study (Miller 2012). Feedback on progress towards goals were provided graphically for participants (receiving services) in the case of three studies (Bell 2003; Duncan 2003; Richardson 2007). In one further study 'goal notebooks' were used to enhance discussions between patients and therapists about goals, but it was unclear whether this included measurement and monitoring of progress towards goals (Gagné 2003). The remaining 22 studies included in this review did not report on the use of strategies to monitor progress toward goals during the course of the intervention.

\section{'Usual care' as a comparison intervention}

Fourteen studies examined a structured approach to goal setting, with or without strategies to enhance goal pursuit, in comparison to 'usual care' where some goals may have been set but no structured goal setting approach was followed (Comparison 2 in this review; see Included studies: Comparison groups). Of these 14 studies, 5 provided at least some details regarding how goal setting in usual care was undertaken (Arnetz 2004; Asenlof 2005; Cheng 2012; Holliday 2007; Ostelo 2003), permitting analysis of how goal setting in the intervention arms of these studies actually differed from goal setting in the control arms. In two of these studies, treatment fidelity in the usual care group was formally assessed to ensure that the control intervention did indeed differ from the experimental intervention in the manner anticipated (Asenlof 2005; Ostelo 2003). For the other nine studies, goal setting in the usual care groups was characterised primarily by not involving the structured approaches to goal setting that the experimental groups received (Gagné 2003; Hart 1978; Jonsdottir 2012; LaFerriere 1978; McPherson 2009; Oestergaard 2012; Parsons 2012; Taylor 2012; Woltmann 2011). Despite this ambiguity, the usual care groups were broadly presented as having less patient involvement in goal selection, being less person-centred, and being less focused on personally meaningful activities that patients wanted to pursue. In three studies, the usual care interventions were also presented as having differed from the experimental interventions because the 
experimental intervention included elements explicitly targeting behavioural change to help participants pursue goals when the usual care interventions did not (Asenlof 2005; McPherson 2009; Ostelo 2003). The specific approach to behavioural change differed from study to study, however. In Asenlof 2005, the experimental group received individual functional behavioural analysis in which behavioural barriers to goal achievement were identified and strategies were implemented to address these. In McPherson 2009, the additional behavioural intervention received by the experimental group focused on improving self-regulation of goaloriented behaviour. In Ostelo 2003, the experimental groups were prescribed graded activity to achieve selected goals, with this intervention being based on contingency management and operant conditioning theory.

\section{Excluded studies}

Of the studies that we excluded from the review, 62 required additional discussion between the review authors before a consensus was reached regarding their exclusion. These are listed in the Characteristics of excluded studies. We gave more than one reason for exclusion for some of these studies but, broadly speaking, we excluded 23 studies because it was not possible to separate the effects of goal setting or strategies to enhance goal pursuit from other (frequently broader) interventions being provided to the experimental group but not the control group. Examples of broader goal-directed interventions included: therapeutic coaching, counselling, work skills training, cognitive behavioural therapy, mind- fulness meditation, self-management skills training, and community rehabilitation.

We excluded an additional 18 studies because they did not involve, or it could not be confirmed that they involved, a population of people with disability acquired in adulthood. Among this group of excluded studies were trials involving goal setting interventions for people with subclinical health concerns (e.g. subclinical weight problems; subclinical high alcohol consumption), healthy older adults in residential care, and adults with congenital conditions or conditions most commonly acquired in childhood. We excluded 13 additional studies because they did not use an RCT or quasiRCT study design. We excluded 3 studies for other reasons. These other reasons included comparing a goal setting intervention with a different behavioural intervention (specifically instructions to attend to pain sensations and modify activity levels accordingly) (Stenstrom 1994), conducting the goal setting intervention and outcome measurement in a single clinical session (Wood 2012), and investigating a type of goal setting that did not align with the definition of 'rehabilitation goals' used for the purposes of this review (Adair 2013).

\section{Risk of bias in included studies}

We present 'Risk of bias' information in Characteristics of included studies and Figure 1. There were no noticeable trends in the 'Risk of bias' assessments when studies were grouped by comparison type, so the summary of information on risk of bias presented below is organised at the level of all included studies. 
Figure I. Risk of bias summary: review authors' judgements about each risk of bias item for each included study.

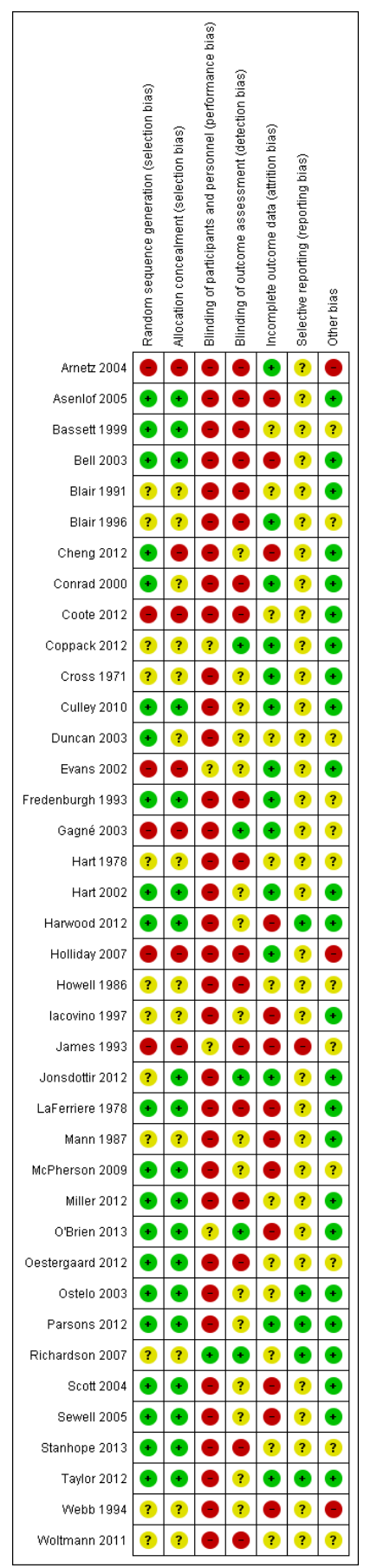

Goal setting and strategies to enhance goal pursuit for adults with acquired disability participating in rehabilitation (Review) 


\section{Allocation}

We judged 21 trials (54\% of included studies) as having low risk of bias for random sequence generation (Asenlof 2005; Bassett 1999; Bell 2003; Cheng 2012; Conrad 2000; Culley 2010; Duncan 2003; Fredenburgh 1993; Hart 2002; Harwood 2012; LaFerriere 1978; McPherson 2009; Miller 2012; O’Brien 2013; Oestergaard 2012; Ostelo 2003; Parsons 2012; Scott 2004; Sewell 2005; Stanhope 2013; Taylor 2012). Information was insufficient to permit a decision in relation to 12 trials (31\% of included studies) (Blair 1991; Blair 1996; Coppack 2012; Cross 1971; Hart 1978; Howell 1986; Iacovino 1997; Jonsdottir 2012; Mann 1987; Richardson 2007; Webb 1994; Woltmann 2011). We judged six trials $(15 \%)$ to be at high risk of bias due to problems with random sequence generation (Arnetz 2004; Coote 2012; Evans 2002; Gagné 2003; Holliday 2007; James 1993). These six studies employed a quasi-RCT design, whereby a systematic method had been used for group allocation (e.g. alternate allocation or allocation based on room assignment or date of admission) or where group allocation had been manipulated in an attempt to balance the groups on the basis of preselected patient characteristics or to address patient preferences for group allocation.

With regards to allocation concealment, we judged 19 trials (49\% of included studies) as having low risk of bias (Asenlof 2005; Bassett 1999; Bell 2003; Culley 2010; Fredenburgh 1993; Hart 2002; Harwood 2012; Jonsdottir 2012; LaFerriere 1978; McPherson 2009; Miller 2012; O’Brien 2013; Oestergaard 2012; Ostelo 2003; Parsons 2012; Scott 2004; Sewell 2005; Stanhope 2013; Taylor 2012) and seven trials (18\% of included studies) to be at high risk of bias (Arnetz 2004; Cheng 2012; Coote 2012; Evans 2002; Gagné 2003; Holliday 2007; James 1993). The remaining 13 trials (33\% of included studies) provided insufficient information to inform judgements (Blair 1991; Blair 1996; Conrad 2000; Coppack 2012; Cross 1971; Duncan 2003; Hart 1978; Howell 1986; Iacovino 1997; Mann 1987; Richardson 2007; Webb 1994; Woltmann 2011).

\section{Blinding}

Blinding of participants and personnel was not feasible in the majority (32/39) of studies because the goal setting intervention required active involvement of both parties in order to be correctly implemented. In the case of three studies, the delivery of the goal setting intervention was automated by the use of technology (phones, computers, and portable voice organisers), removing the need to blind personnel to group allocation (Culley 2010; Hart 2002; Richardson 2007). In the case of one of these studies, participants were also blinded to the aspect of goal setting under investigation (the type of goal set), so this study was effectively blinded for both participants and personnel (Richardson 2007).
In two additional studies, while the participants in the experimental and control group were actively involved in the delivery of the intervention, they were not informed of the specific details regarding the hypothesis under investigation, partially blinding them to the experimental and control conditions (Coppack 2012; Evans 2002). Likewise, in yet another study, while therapists were aware of the differences in treatment protocol for the groups in the trial, they were not informed about the experimental hypothesis, partially blinding them to the anticipated patient response to the study conditions (James 1993). In two studies, different personnel were used for goal setting versus the other aspects of the rehabilitation intervention (e.g. exercise therapy), with those involved in goal setting not blinded to group allocation but those involved in delivery of therapy blinded to group allocation (Evans 2002; O’Brien 2013).

In terms of blinding for outcome assessment, five studies used adequate methods for blinding those people involved in outcome data collection (Coppack 2012; Gagné 2003; Jonsdottir 2012; O’Brien 2013; Richardson 2007). In ten studies the personnel involved in outcome data collection were not blinded to study group allocation (Arnetz 2004; Asenlof 2005; Bassett 1999; Blair 1991; Blair 1996; Holliday 2007; Howell 1986; James 1993; Stanhope 2013; Woltmann 2011). In eight other studies, outcome assessors were ostensibly blinded to group allocation but the overall risk of detection bias was deemed 'high' due to the outcome data collection being heavily dependent on self-report by participants who were not (or could not be) blinded to group allocation, or due to other problems with maintaining the blinding of outcome assessors (Bell 2003; Conrad 2000; Coote 2012; Fredenburgh 1993; Hart 1978; LaFerriere 1978; Miller 2012; Oestergaard 2012). For the remaining 16 studies, the risk of detection bias was unclear. For four studies, this was because insufficient information was reported on the possible blinding of outcome assessors (Cross 1971; Iacovino 1997; Mann 1987; Webb 1994). For 12 studies, the risk of detection bias was unclear because, while the outcome assessor had been blinded to group allocation, the collection of data had been partially based on self-report by participants who had not been blinded to group allocation (Cheng 2012; Culley 2010; Duncan 2003; Evans 2002; Hart 2002; Harwood 2012; McPherson 2009; Ostelo 2003; Parsons 2012; Scott 2004; Sewell 2005; Taylor 2012).

Overall, the majority of studies were at high risk of performance bias due to the nature of the intervention under investigation. However, $13 \%$ (5/39) of studies were at low risk of detection bias, 41\% (16/39) had unclear risk of detection bias (16/39), and $46 \%(18 / 39)$ were at high risk of detection bias. Seventeen of the included studies $(44 \% ; 17 / 39)$ were deemed to have high risk for both performance and selection bias, while only one study (Richardson 2007) was deemed to be at low risk for both perfor- 
mance and selection bias.

\section{Incomplete outcome data}

Of the 39 included studies, 13 reported losses to follow-up of less than $5 \%$, one study reported loss to follow-up of 7\%, 11 reported losses to follow-up of $10 \%$ to $20 \%, 12$ reported losses to follow-up of higher than $20 \%$, and for the remaining two studies there was insufficient information available to judge the number of participants lost to follow up. There was no compelling evidence of an imbalance in losses to follow-up across the intervention groups in any study, except for Woltmann 2011, where there was slightly higher attrition in the intervention group (17.5\%; $7 / 40)$ versus the control group $(10 \% ; 4 / 40)$.

For the studies reporting moderate to high loss of participants to follow-up (i.e. $10 \%$ to $20 \%$ ), three reported following an intention-to-treat analysis approach (Asenlof 2005; Bell 2003; Ostelo 2003). Bell 2003 reported using an imputation method of analysis, bringing the last observed outcome forward for all missing data (raising the risk of attrition bias). Ostelo 2003 used a single value imputation method, substituting missing data with mean value, negative values and positive values if the reasons for participants dropping out were unexplained, an aggravation in symptoms, or a complete remission of symptoms, respectively. Asenlof 2005 compared multiple methods for management of missing data in their analysis: imputation methods from last observed outcome, substitution with the worst 10 th percentile, and omission of missing data from the analysis. As these different methods were not found to alter the findings of the study, data from the latter approach were reported.

Overall, there was a spread of low to high risk of attrition bias, with 36\% (14/39) of all studies being at low risk of attrition bias, $31 \%(12 / 39)$ having unclear risk of attrition bias, and 33\% (13/ 39) being at high risk of attrition bias.

\section{Selective reporting}

Five studies had a protocol published in a peer-reviewed journal or clinical trial registry prior to the trial being conducted (Harwood 2012; Ostelo 2003; Parsons 2012; Richardson 2007; Taylor 2012). For these studies we could confirm that there was no evidence of selective reporting. For the majority of trials $(85 \% ; 33 / 39)$ no published protocol could be identified, which meant that the risk of reporting bias for these studies was unclear. For four of these studies, data on multiple outcomes were collected and/or collected at multiple time points, with no named primary outcome, resulting in multiple tests for statistical significance and/or incomplete reporting of all results (Arnetz 2004; Duncan 2003; Iacovino 1997; Scott 2004). Cross 1971 reported some statistically significant findings, but appeared to have adjusted their analysis (e.g. combining groups; reporting change scores when end scores were not significant) in order to find these. Unadjusted SDs for the main outcomes in Cross 1971 were not reported. Finally, James 1993 was deemed to be at high risk of reporting bias as outcome data were explicitly dropped from the analysis when it was considered to be outlying.

\section{Other potential sources of bias}

For 26 of the included studies (67\%) there was low risk of additional sources of bias, including low risk of cross-group contamination. For another ten studies (26\%), other sources of bias were noted that presented an unclear level of risk. Of these, seven were deemed to have had potential for cross-group contamination, where the risk arising from this was unclear (Bassett 1999; Blair 1996; Fredenburgh 1993; Gagné 2003; Hart 1978; Howell 1986; McPherson 2009). For two studies, participants in the experimental groups appeared to receive much higher levels of contact time with the clinical providers than those in the control groups, resulting in an unclear level of additional risk of bias (Webb 1994; Woltmann 2011). Oestergaard 2012 used a novel, untested outcome measure to compare their two study groups where the construction of the measure itself appeared to favour the experimental intervention. Furthermore, Oestergaard 2012 appeared to introduce an outcome measure (the Dallas Pain Questionnaire) during the course of the study rather than decide on all outcomes and timing of data collection a priori, again introducing an unclear risk of bias.

Three studies (8\%) were deemed to have involved a high risk of additional sources of bias (Arnetz 2004; Holliday 2007; Webb 1994). Of these, Arnetz 2004 used goal attainment as a main outcome measure to evaluate the effect of goal setting, but did not report when the therapists in each study group set the goals for therapy (i.e. before or after randomisation). Similarly, Webb 1994 used GAS as their only outcome measure. While the scoring of these GAS outcomes was reported as being undertaken by a blinded assessor, the construction of the individualised GAS scales in Webb 1994 was completed with involvement from an unblinded treating therapist after randomisation and allocation of participants to the study groups. Finally, Holliday 2007 reported the involvement of all staff in the study setting (an inpatient rehabilitation ward) in treating participants in both the experimental and control groups, sometimes concurrently, significantly increasing the risk of crossgroup contamination.

\section{Effects of interventions}

See: Summary of findings for the main comparison Goal setting with or without strategies to enhance goal pursuit compared to no goal setting for adults with acquired disability participating in rehabilitation; Summary of findings $\mathbf{2}$ Structured goal setting with or without strategies to enhance goal pursuit compared to 'usual care' that involved some goal setting but where no structured approach was followed for adults with acquired disability participating in rehabilitation 
See also Data and analyses for pooled analyses; Table 2 for information about the selection and management of data for pooled analyses; Table 3 for data from single studies that were not pooled.

\section{Comparison I: Structured goal setting with or without strategies to enhance goal pursuit versus no goal setting}

\section{Primary outcomes}

Health-related quality of life or self-reported emotional status Eight studies $(\mathrm{n}=446)$ in this comparison group reported data on health-related quality of life or self-reported emotional status that could be pooled in a meta-analysis using standard effect sizes
(Blair 1991; Coote 2012; Duncan 2003; Evans 2002; Fredenburgh 1993; Harwood 2012; Scott 2004; Sewell 2005). The meta-analysis showed an increase in health-related quality of life or selfreported emotional status when some form of goal setting (plus or minus strategies to enhance goal pursuit) was used in comparison to no goal setting (standard mean difference (SMD) 0.53 , $95 \%$ confidence interval (CI) 0.17 to 0.88 ; Analysis 1.1 ; Figure 2). This mean effect estimate suggests a moderate clinical effect size in favour of goal setting (Higgins 2011). Substantial statistical heterogeneity in outcome was observed, but with seven out of the eight effect sizes favouring goal setting. Insufficient studies existed to permit meaningful subgroup analysis to further explore reasons for the heterogeneity. Removal of studies at the greatest risk of bias (Coote 2012; Evans 2002) resulted in a minor reduction in the pooled SMD and a widening of the $95 \% \mathrm{CI}$, but the result remained in favour of goal setting (SMD $0.45,95 \%$ CI 0.02 to 0.87).

\section{Figure 2. Forest plot of comparison: I Goal setting (with or without strategies to enhance goal pursuit) versus no goal setting, outcome: I.I Health related quality of life or self-reported emotional status.}

\begin{tabular}{|c|c|c|c|c|c|c|c|}
\hline \multirow[b]{2}{*}{ Study or Subgroup } & \multicolumn{3}{|c|}{ Goal setting } & \multicolumn{3}{|c|}{ No goal setting } & \multirow[b]{2}{*}{ Weight } \\
\hline & Mean & SD & Total & Mean & SD & Total & \\
\hline Blair 1991 & -1.377 & 0.924 & 53 & -1.855 & 0.766 & 26 & $14.7 \%$ \\
\hline Coote 2012 & -26.2 & 13.3 & 26 & -32.5 & 11.9 & 29 & $13.7 \%$ \\
\hline Duncan 2003 & -25.4 & 21.6 & 7 & -33.5 & 22.6 & 7 & $7.2 \%$ \\
\hline Evans 2002 & 14.46 & 2.73 & 13 & 11.7 & 2.29 & 26 & $11.0 \%$ \\
\hline Fredenburgh 1993 & 12.08 & 26.1 & 15 & 4.79 & 20.63 & 15 & $10.9 \%$ \\
\hline Harwood 2012 & 44.8 & 10.4 & 38 & 35.9 & 10.1 & 31 & $14.4 \%$ \\
\hline Scott 2004 & 25.02 & 3.63 & 15 & 20.79 & 4.78 & 24 & $11.5 \%$ \\
\hline Sewell 2005 & 0.62 & 1.41 & 63 & 0.89 & 1.29 & 58 & $16.6 \%$ \\
\hline Total $(95 \% \mathrm{Cl})$ & & & 230 & & & 216 & $100.0 \%$ \\
\hline
\end{tabular}

Test for overall effect: $Z=2.91(P=0.004)$

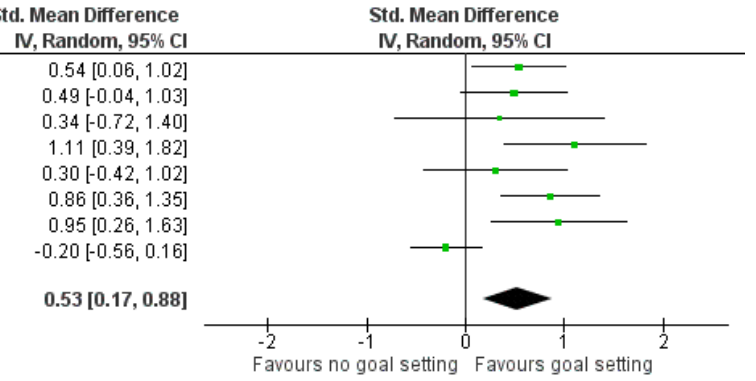

Favours no goal setting Favours goal setting
To test for the influence of the one unadjusted cluster-RCT in this analysis we removed Blair 1991 from the pooled data. This widened the $95 \%$ CI for the SMD only a little (SMD 0.53, 95\% CI 0.11 to 0.95 ), so we judged that not including an adjustment for clustering in this RCT had minimal effect on the result of the meta-analysis.

Two additional studies in this comparison group collected data on health-related quality of life, but could not be included in the meta-analysis because no means or SDs were reported (Bell 2003, $\mathrm{n}=74,15 \%$ attrition; Iacovino $1997, \mathrm{n}=68,32 \%$ attrition). Both of these studies reported no statistically significant results for these outcomes.

\section{Participation outcomes as defined by the ICF}

Four studies in this comparison group reported data on measures of participation as defined by the ICF (WHO 2001a), but the measures used were too dissimilar to permit meta-analysis (Bell
2003; Howell 1986; Iacovino 1997; Sewell 2005). Bell 2003 ( $\mathrm{n}=$ $11 ; 15 \%$ attrition) reported a difference in favour of their goal setting intervention in terms of improvements in work performances as measured by Total Work Behaviour Inventory scores (mean difference (MD) 16.0, 95\% CI 4.22 to 27.78). Howell 1986 ( $n=27$; $11 \%$ attrition) did not report SDs, but stated that there were no statistically significant differences on the Griffiths Work Performance Scale between the treatment and control groups at the end of intervention. Iacovino 1997 ( $n=68 ; 32 \%$ attrition) reported no difference between goal setting and non-goal setting groups in terms of numbers returning to work three to six months after the intervention (RR $0.78,95 \%$ CI 0.56 to 1.08). Sewell 2005 ( $n=180 ; 33 \%$ attrition) also reported no difference between goal setting and non-goal setting groups in self-reported satisfaction with occupational performance after seven weeks of pulmonary 
rehabilitation (MD - $0.23,95 \% \mathrm{CI}-0.93$ to 0.47 ).

\section{Activity outcomes as defined by the ICF}

Four studies $(\mathrm{n}=223)$ reported data from measures of activity as defined by the ICF (WHO 2001a), which we combined in a meta-analysis using standard effect sizes (Duncan 2003; Harwood 2012; O'Brien 2013; Sewell 2005). The meta-analysis showed no difference in activity levels when some form of goal setting (plus or minus strategies to enhance goal pursuit) was used in comparison to no goal setting (SMD 0.04, 95\% CI -0.22 to 0.31; Analysis 1.2; Figure 3). There was no evidence of statistical heterogeneity in the observed outcomes.

Figure 3. Forest plot of comparison: I Goal setting (with or without strategies to enhance goal pursuit) versus no goal setting, outcome: I.2 Activity - ability.

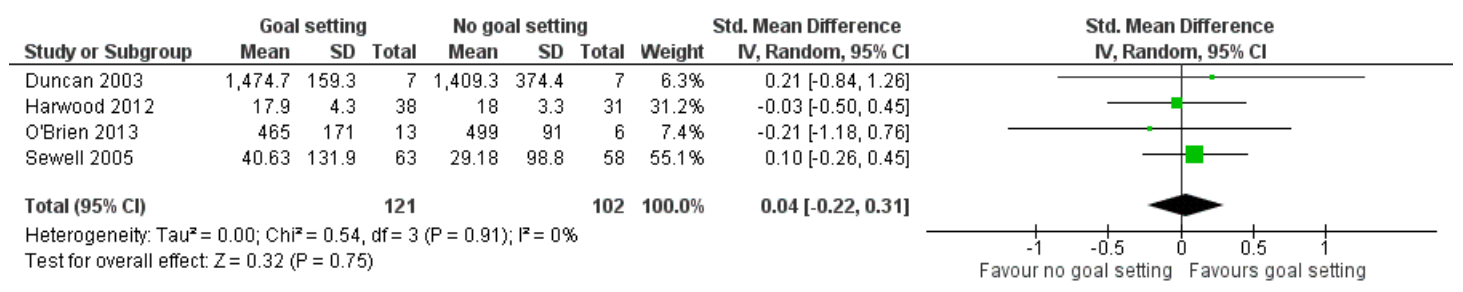

\section{Secondary outcomes}

\section{Outcomes at the level of body structure or body function as defined by the ICF}

Data from measures at the level of body function as defined by the ICF (WHO 2001a) were reported in five studies (Bassett 1999; Mann 1987; Harwood 2012; Coppack 2012; Duncan 2003). The measures used were too dissimilar to permit meta-analysis, and thus are presented descriptively instead.

Bassett 1999 ( $\mathrm{n}=74 ; 15 \%$ attrition) reported no difference in self-reported symptom relief. These data were collected on a 3point Likert-type scale, but analysed as a continuous variable (presented as means and SDs). Insufficient information about this data set was reported to treat it as categorical data as per our protocol. Mann 1987 ( $\mathrm{n}=66$; 15\% attrition) reported no difference between their groups receiving self-management education with goal setting and self-management education without goal setting in terms of urinary sodium output three months after the training (MD $16.0 \mathrm{mmol} / 24 \mathrm{~h}, 95 \% \mathrm{CI}-19.2$ to 51.2 ) or in terms of systolic blood pressure at three months following the training (MD -2.60 mmHg, 95\% CI -13.73 to 8.53). Similarly, Harwood 2012 ( $\mathrm{n}=85$ in the 'Take Charge' and control groups combined; $19 \%$ attrition) reported no difference between their 'Take Charge' (goal setting) group and control group on the basis of systolic blood pressure (MD $-3.5 \mathrm{mmHg}, 95 \% \mathrm{CI}-12.15 \mathrm{mmHg}$ to 5.15 $\mathrm{mmHg}$ ).

Coppack 2012 ( $\mathrm{n}=48$; no attrition) reported a difference between their goal setting and control groups both at baseline and at the end of treatment in terms of back muscle endurance (as measured by the Biering-Sørensen test), with the goal setting group performing better on this test at both time points. However, Coppack 2012 reported no statistically significant difference between their three groups (one goal setting group and two control groups) in terms of change in back muscle endurance scores between baseline and treatment end on the basis of multivariate analysis of covariance. As no SDs were reported for change scores at a group level in Coppack 2012, a 95\% CI could not be reported in this review for the MD in change in the Biering-Sørensen test.

Similarly, Duncan 2003 ( $n=16 ; 6 \%$ attrition) reported on data indicating a difference between their goal setting and control groups both at baseline and 24 weeks after study enrolment in terms of maximum ventilated oxygen, with the control group achieving higher maximum ventilated oxygen at both time points. However, a $95 \% \mathrm{CI}$ could not be calculated for this review for the MD in change scores for maximum ventilated oxygen as no SDs were reported for change scores at a group level. Duncan 2003 also reported no differences between their two groups at 24 weeks after 
study enrolment on the basis of self-reported dyspnoea as measured by the Baseline Dyspnea Index (MD 1.6, 95\% CI - 0.13 to 3.33 ) or fatigue measured by the Piper Fatigue Scale (MD -0.4, $95 \%$ CI -2.29 to 1.49 ).

Finally, one study also collected data from a criterion-reference achievement test (Mann 1987; $\mathrm{n}=66 ; 15 \%$ attrition). The test was designed for participants with hypertension and included questions testing knowledge of the condition and its management as well as affective responses (e.g. feelings of support; feelings of selfcompetence). Three months after enrolment in the study, there was no difference between the dietary education plus goal setting group compared to the group receiving dietary education without goal setting on this test (MD 1.7 on a test with a maximum score of $23,95 \%$ CI -0.22 to 3.62 ).

Patient self-belief and engagement in rehabilitation

\section{a) Adherence and engagement in rehabilitation}

Nine studies $(n=369)$ in this comparison group reported data on measures of patient engagement that could be pooled in a meta-analysis using standard effect sizes (Bassett 1999; Bell 2003; Coppack 2012; Cross 1971; Duncan 2003; Evans 2002; Iacovino 1997; Mann 1987; O’Brien 2013). The meta-analysis showed no difference in patient engagement in rehabilitation when some form of goal setting (plus or minus strategies to enhance goal pursuit) were used in comparison to no goal setting (SMD 0.30, 95\% CI -0.07 to 0.66 ; Analysis 1.3; Figure 4). There was evidence of substantial heterogeneity in the observed outcomes both in terms of the size and direction of effects. Insufficient studies existed to permit meaningful subgroup analysis to further explore reasons for the heterogeneity.

Figure 4. Forest plot of comparison: I Goal setting (with or without strategies to enhance goal pursuit) versus no goal setting, outcome: I.3 Engagement in rehabilitation.

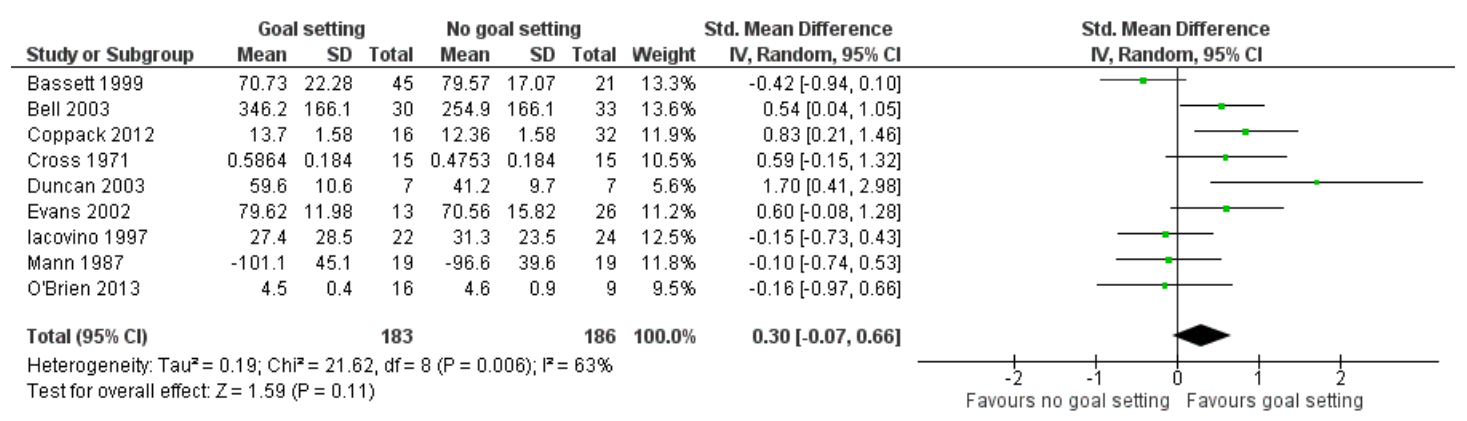

One additional study in this comparison group reported on engagement in rehabilitation, but could not be included in the metaanalysis because no means or SDs were reported (Howell 1986; $\mathrm{n}=$ $27 ; 11 \%$ attrition). This study reported no statistically significant results for this outcomes.

One further study ( $\mathrm{n}=367 ; 30 \%$ attrition) in this comparison group reported on patient adherence as a dichotomous variable only, reporting the percentage of participants with a mental health condition who adhered to a prescribed medication regime (Stanhope 2013). These data were calculated as overall rates of medication adherence per month for 11 months. To accommodate unit of analysis issues in this-cluster-RCT, Stanhope 2013 reported using logistic regression models while including a random effect for site to calculate overall medication adherence. The reported odds ratio $(\mathrm{OR})$ for medication adherence over time for the whole study population was 1.13 (95\% CI 1.08 to 1.19) (author communication), indicating a very small effect size in favour of the goal setting intervention.

\section{b) Self-efficacy}

Three studies $(n=108)$ in this comparison group reported data from measures of task-specific self-efficacy following rehabilitation that we could combine in a meta-analysis using standard effect sizes (Coppack 2012; Evans 2002; O’Brien 2013). The meta-analysis showed a difference in self-efficacy at the end of rehabilitation in favour of goal setting (with and without strategies to enhance goal pursuit) in comparison to no goal setting (SMD 1.07, 95\% CI 0.64 to 1.49 ; Analysis 1.4). This mean effect estimate suggests a large effect size (Higgins 2011). There was little evidence of heterogeneity in these data. 
One additional study in this comparison group reported collecting data on general self-efficacy, but reported no means or SDs for this outcome (Scott 2004; $\mathrm{n}=88 ; 36 \%$ attrition). This study reported no statistically significant results for this outcomes.

\section{Individual goal attainment}

Three studies reported on individual goal attainment as a study outcome (Blair 1991; Blair 1996; Howell 1986). In these studies goals were set by researchers for the participants in both the intervention and control groups, but the control group participants (and, in the case of Howell 1986 their treating health professionals) were not made aware of these goals and the goals were not used during the delivery of rehabilitation interventions. In Howell 1986 these goals were thus called 'theoretical' goals. In all these studies goal attainment was reported as GAS scores and was treated as interval data only (reported as means and SDs) rather than ordinal data (as required by our protocol; see Measures of treatment effect), so no results could be extracted for the purposes of this review. However, Howell 1986 ( $n=27 ; 11 \%$ attrition) also reported on goal attainment as a dichotomous variable, categorising participants as either having reached 'high' goal achievement or 'low' goal achievement on their theoretical GAS scale. While it was not clearly reported what 'high' goal achievement represented in this study, Howell 1986's data indicated no difference between the two groups at study end when categorised in this way (risk ratio (RR) $0.97,95 \%$ CI 0.52 to 1.80 ).

\section{Evaluation of care}

No data were reported in studies in this comparison group on evaluation of care (such as satisfaction with service delivery).

\section{Service delivery level}

One study in this comparison group, set in the context of physiotherapy for musculoskeletal disorders, reported on the number of treatment sessions required to achieve symptom relief (Bassett $1999 ; \mathrm{n}=74 ; 11 \%$ attrition). No differences were founded when the pooled means and SDs for the two goal setting groups in this study (participant-physiotherapist collaborative goal setting and the physiotherapist-mandated goal setting) were compared to the control group who received no goal setting on the basis of the number of treatment sessions required to achieve symptom relief (MD -0.73 sessions, $95 \%$ CI -5.85 to 4.39 ).

\section{Adverse events}

One study (Harwood 2012; $\mathrm{n}=172$ ) reported on the number of participants in each group who died prior to final data collection. No difference in risk of death was observed between the goal setting ('Take charge') group and control group in this study (RR 0.68, $95 \%$ CI $0.20,2.35$ ). However, as the $95 \%$ CIs for this comparison are wide, it is not possible to rule out differences in mortality between the two groups without considerably more data.

\section{Comparison 2: Structured approach to goal setting with or without strategies to enhance goal pursuit versus 'usual care' that may have involved some goal setting but where no structured approach was followed}

\section{Primary outcomes}

\section{Health-related quality of life and self-reported emotional status}

Five studies $(\mathrm{n}=441)$ in this comparison group reported data on health-related quality of life or self-reported emotional status that could be pooled in a meta-analysis using standard effect sizes (Cheng 2012; LaFerriere 1978; Ostelo 2003; Parsons 2012; Taylor 2012). The meta-analysis showed no difference in healthrelated quality of life or self-reported emotional status when a structured approach to goal setting (plus or minus strategies to enhance goal pursuit) was compared to usual care without a structured approach to goal setting (SMD 0.18, 95\% CI -0.19 to 0.55; Analysis 2.1; Figure 5). Substantial heterogeneity was observed. Insufficient studies existed to permit meaningful subgroup analysis to explore reasons for the heterogeneity. Removal of studies at the greatest risk of bias (Cheng 2012) shifted the SMD a little more in favour of structured goal setting, and widened the CIs for this estimate a little, but did not change the conclusion of this analysis (SMD $0.27,95 \%$ CI -0.16 to 0.70 ). Removal of Cheng 2012 also suggested that lack of adjustment for the effects of clustering in this cluster-RCT had minimal influence on the outcome of this meta-analysis. 
Figure 5. Forest plot of comparison: 2 Structured goal setting (with or without strategies to enhance goal pursuit) versus no structured goal setting, outcome: 2 .I Health related quality of life or self-reported emotional status.

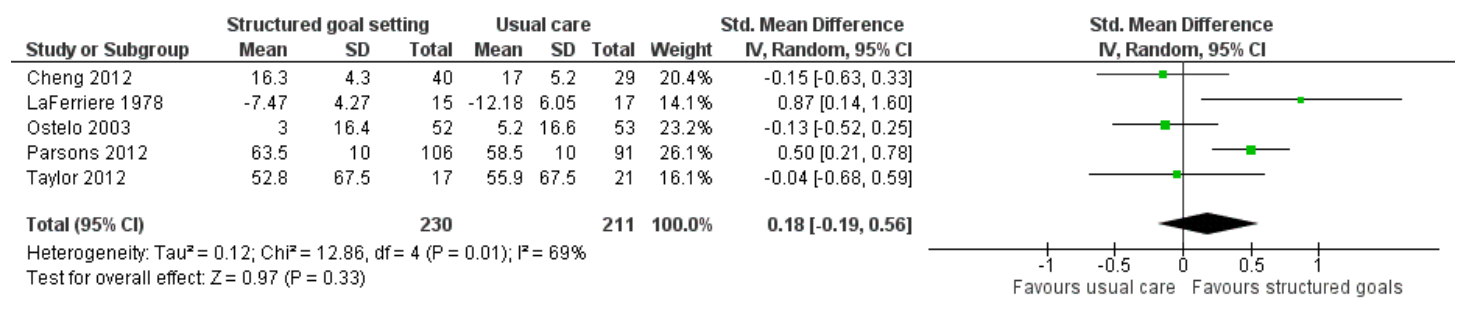

One additional study in this comparison group, Holliday 2007 (a quasi-RCT; $\mathrm{n}=201$; no attrition), reported on health-related quality of life as measured by the General Health Questionnaire, but could not be included in the meta-analysis because no means or SDs were reported. Nonetheless this study reported a statistically significant difference in favour of their usual care group for healthrelated quality of life ( $\mathrm{P}=0.037$; no effect size reported).

In addition to studies that reported on continuous outcome data, one study in this comparison group reported on overall 'satisfaction with daily living' using ordinal data (Asenlof 2005). When these data were dichotomised, the participants in the structured goal setting intervention were more likely to report being more satisfied or much more satisfied with their life (versus no difference or less satisfied with their life) compared to participants in the usual care group three months after the intervention (RR 1.44, 95\% CI 1.09 to 1.88$)$. However, this difference did not remain two years after intervention (RR 1.27, 95\% CI 0.94 to 1.70 ).

\section{Participation outcomes as defined by the ICF}

One study, Holliday 2007 (a quasi-RCT; n = 201; no attrition), reported on outcomes from a single measure of participation as defined by the ICF (WHO 2001a) - the London Handicap Scale. However, while the authors of this study reported that there was no difference between the two study groups on this measure, no means or SDs were published.

\section{Activity outcomes as defined by the ICF}

Four studies $(\mathrm{n}=277)$ in this comparison group reported data on activity outcomes as defined by the ICF (WHO 2001a) that could be pooled in a meta-analysis using standard effect sizes (Asenlof 2005; Cheng 2012; Ostelo 2003; Taylor 2012). The meta-analysis showed no difference in activity levels when a structured approach to goal setting (plus or minus strategies to enhance goal pursuit) was compared to usual care without a structured approach to goal setting (SMD 0.17, 95\% CI -0.15 to 0.49; Analysis 2.2). Moderate heterogeneity was observed. Insufficient studies existed to permit meaningful subgroup analysis to further explore reasons for the heterogeneity. Removal of studies at the greatest risk of bias (Cheng
2012) reduced the SMD (i.e. less in favour of goal setting) and widened the $95 \%$ CI for this estimate a little, but did not change the conclusion of this analysis (SMD $0.07,95 \%$ CI - 0.30 to 0.44 ). Removal of Cheng 2012 also suggested that lack of adjustment for the effects of clustering in this cluster-RCT had minimal influence on the outcome of this meta-analysis.

One additional study in this comparison group, Holliday 2007 (a quasi-RCT; $\mathrm{n}=201$; no attrition), reported on activity levels as measured by the Functional Independence Measure, but could not be included in the meta-analysis because no means or SDs were reported. This study reported no statistically significant difference between their structured goal setting and usual care groups.

Two further studies reported on activity data as median and percentile scores only, treating the data as ordinal measures (Gagné 2003; Oestergaard 2012). Gagné 2003 ( $\mathrm{n}=31$; no attrition) reported a difference in favour of structured goal setting in comparison to usual care in Functional Independence Measure subscores for upper body dressing after two weeks of occupational therapy (Mann-Whitney U test, $\mathrm{P}<0.019$ ), but not for five other subscores on activities of daily living: eating, grooming, lower-body dressing, toileting, and bathing. Oestergaard 2012 ( $\mathrm{n}=87 ; 31 \%$ attrition) reported no differences between their structured goal setting and usual care groups in terms of self-rated performance and satisfaction with performance on a list of 18 activities of daily living when using Wilcoxon rank sum to test for differences.

\section{Secondary outcomes}

Outcomes at the level of body structure or body function as defined by the ICF

Three studies reported outcomes at the level of body function as defined by the ICF (WHO 2001a) that could be reported descriptively but not pooled in a meta-analysis (Asenlof 2005; Oestergaard 2012; Ostelo 2003). Asenlof 2005 ( $n=122$; 47\% attrition) reported on maximum pain and pain control on 10-point Likert-type scales two years after physical therapy for a persistent 
pain problem. When the baseline values for these measures were used as covariates in the analyses, Asenlof 2005 reported that there was a difference in favour of the structured goal setting intervention for maximum pain but not pain control. When the reported data were analysed without adjustment for baseline values however, there was no difference for either measure at two years (maximum pain MD 1.30, 95\% CI -0.24 to 2.84). Oestergaard 2012 ( $\mathrm{n}=87$; $31 \%$ attrition at three years) reported on pain, as measured by the Dallas Pain Questionnaire, experienced by participants three years after multidisciplinary rehabilitation for lumbar spinal fusion for degenerative disc disease. This was reported as ordinal data, with a Wilcoxon rank sum test identifying no difference between the structured goal setting and usual care groups for total Dallas Pain Questionnaire scores at three years $(\mathrm{P}=0.38)$. Ostelo 2003 ( $\mathrm{n}=$ $105 ; 11 \%$ attrition) reported on three measures of body function (severity of back pain, severity of sciatica, and range of lumbar spine movement) in people following physiotherapy for persistent back pain following lumbar disc surgery. No differences were observed between the structured goal setting and usual care group for any of these measures of body function, with change in range of lumbar spine movement (in degrees) being the measure with the median effect size (MD $-1.20^{\circ}, 95 \%$ CI $-9.66^{\circ}$ to $7.26^{\circ}$ )

\section{Patient self-belief and engagement in rehabilitation}

\section{a) Adberence and engagement in rehabilitation}

Only one study (LaFerriere 1978; $\mathrm{n}=65 ; 51 \%$ attrition) reported on measures of patient engagement in rehabilitation, specifically therapist-rated scores and patient-rated scores of patient motivation for people with mental health conditions participating in a series of individual behaviour therapy or psychotherapy sessions. LaFerriere 1978 reported a small difference in favour of structured goal setting for patient-rated motivation (MD 1.40, 95\% CI 0.43 to 2.37 ), but not for therapist-rated scores of motivation (MD $0.48,95 \%$ CI -0.41 to 1.37 ) after completion of the therapy.

\section{b) Self-efficacy}

Two studies $(\mathrm{n}=134)$ reported on general self-efficacy as an outcome following rehabilitation (Asenlof 2005; Cheng 2012). When combined in a meta-analysis using standard effect sizes and the last recorded, unadjusted self-efficacy data from both Asenlof 2005 and Cheng 2012, a difference in favour of the structured goal setting interventions was found (SMD 0.37, 95\% CI 0.02 to 0.71 ; Analysis 2.3), indicative of a small effect size. The accuracy of this estimate is questionable however, given the lack of ability to adjust for clustering in Cheng 2012.

\section{c) Kinesiophobia}

Kinesiophobia was reported in two studies involving people receiving physiotherapy for persistent pain problems (Asenlof 2005; Ostelo 2003). Neither study reported any difference between their structured goal setting group and usual care group in terms of Tampa Scale of Kinesiophobia scores, which were reported as end values for Asenlof 2005 (MD 2.5, 95\% CI -0.69 to 5.69) and change from baseline values in the case of Ostelo 2003 (MD 0.1, $95 \%$ CI -2.33 to 2.53$)$.

\section{Individual goal attainment}

Levels of goal attainment were reported in five studies (Arnetz 2004; Cheng 2012; Hart 1978; Jonsdottir 2012; McPherson 2009). However, the diversity of methods used for collection and reporting of goal attainment data meant that undertaking a metaanalysis on this outcome was not possible. Arnetz 2004 ( $n=77 ; 3 \%$ attrition) reported on the percentages of patients achieving their goals within subgroups of patients with similar types of goals (e.g. people with goals relating to pain, range of movement, strength, balance), but as information was missing on the number of patients in the structured goal setting group versus the usual care group within each subgroup, RRs could not be calculated for most of these data. For Arnetz 2004's data on attainment of goals related to range of movement however, we were able to extrapolate sample sizes from other data within the text and from a $\mathrm{Chi}^{2}$ test reported in the paper. From these we were able to calculate that, for those with range of movement goals, people in the structured goal setting group were more likely to achieve their goals when compared to people within the usual care group (RR 2.32, 95\% CI 1.20 to 4.47). For Cheng 2012 ( $\mathrm{n}=96 ; 28 \%$ attrition), goal attainment was measured as the percentage of goals achieved by each patient within each group (structured goal setting versus usual care). On this basis, participants in the structured goal setting group were found to achieve a higher percentage of their goals than people in the usual care group at 24 weeks after enrolment (MD 36.5\%, $95 \%$ CI $18.43 \%$ to $54.57 \%$; results unadjusted for clustering effects as no intraclass correlations for this measure were available). For Hart 1978, goal attainment was recorded as GAS scores. However these scores were treated as interval data (reported as means and SDs) rather than ordinal data (as required by our protocol; see Measures of treatment effect), so no results could be extracted. Jonsdottir 2012 reported on goal attainment at the level of goals per group in the study, with some participants having more than one goal. As goal attainment was not reported at a participant level, however, no data could be extracted. McPherson 2009 ( $\mathrm{n}=$ 34; $35 \%$ attrition) also reported on goal attainment on the basis of GAS scores. In this case however, raw data for each participant was available from the researchers (author communication), and were dichotomised. On this basis, seven out of 13 participants in the two structured goal setting groups (Goal Management Training and Identity Oriented Goal Mapping) achieved or exceeded at 
least one of their set goals (with each participant having between one and three goals) in comparison to seven out of nine in the usual care group. No significant difference between the groups in terms of goal attainment was observed (RR $0.69,95 \%$ CI 0.38 to 1.28).

Goal recall was also reported in one study (Woltmann 2011; $\mathrm{n}=$ $80 ; 14 \%$ attrition) involving people participating in communitybased interventions for mental health conditions. Recall of goal was evaluated in terms of the percentage of goals that could be reported back to researchers by participants two to four days after a goal planning meeting. In this study, the participants in the structured goal setting group recalled a higher percentage of their goals when compared to participants in the usual care group (MD $18 \%, 95 \%$ CI $3.84 \%$ to $32.16 \%$; results unadjusted for clustering effects as no intraclass correlations for this measure were available).

Evaluation of care
Five studies $(\mathrm{n}=309)$ reported data on satisfaction with care that could be pooled in a meta-analysis using standard effect sizes (Arnetz 2004; Cheng 2012; LaFerriere 1978; Taylor 2012; Woltmann 2011). The meta-analysis showed a statistically significant difference in patient satisfaction in favour of structured goal setting (with and without strategies to enhance goal pursuit) in comparison to usual care (SMD $0.33,95 \%$ CI 0.10 to 0.56 ; Analysis 2.4; Figure 6, indicative of a small effect size) (Higgins 2011). There was no evidence of heterogeneity in these data. When we excluded studies at high risk of bias due to lack of adequate randomisation (Arnetz 2004) or lack of concealment of random allocation (Arnetz 2004; Cheng 2012) from the meta-analysis, the pooled SMD was no longer statistically significant. If we just excluded studies for which we could not account for the effects of clustering (Cheng 2012; Woltmann 2011) the pooled SMD remained statistically significant, albeit with a wider 95\% CI (SMD $0.42,95 \%$ CI 0.03 to 0.82 ).

Figure 6. Forest plot of comparison: 2 Structured goal setting (with or without strategies to enhance goal pursuit) versus no structured goal setting, outcome: 2.4 Satisfaction with service delivery.

\begin{tabular}{|c|c|c|c|c|c|c|c|}
\hline \multirow[b]{2}{*}{ Study or Subgroup } & \multicolumn{3}{|c|}{ Structured goal setting } & \multicolumn{3}{|c|}{ Usual care } & \multirow[b]{2}{*}{ Weight } \\
\hline & Mean & SD & Total & Mean & SD & Total & \\
\hline Arnetz 2004 & 9.6 & 0.67 & 39 & 8.9 & 1.29 & 38 & $24.1 \%$ \\
\hline Cheng 2012 & 79.3 & 5.95 & 46 & 77.4 & 8.1 & 36 & $26.6 \%$ \\
\hline LaFerriere 1978 & 9.07 & 1.22 & 15 & 8.41 & 1.62 & 17 & $10.3 \%$ \\
\hline Taylor 2012 & 80.7 & 60.45 & 17 & 80.16 & 60.45 & 21 & $12.5 \%$ \\
\hline Woltmann 2011 & 3.88 & 0.54 & 40 & 3.78 & 0.56 & 40 & $26.5 \%$ \\
\hline Total $(95 \% \mathrm{Cl})$ & & & 157 & & & 152 & $100.0 \%$ \\
\hline
\end{tabular}

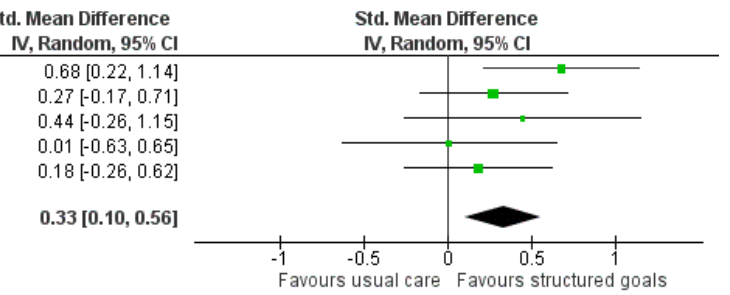

One additional study, Holliday 2007 (a quasi-RCT; $\mathrm{n}=201$; no attrition), reported on participant satisfaction scores, but could not be included in the meta-analysis because no means or SDs were reported. Nonetheless, this study reported a statistically significant difference in favour of structured goal setting when compared to usual care $(\mathrm{P}<0.001)$.

\section{Service delivery level}

Five studies evaluated the extent of use of healthcare services (Asenlof 2005; Cheng 2012; LaFerriere 1978; Oestergaard 2012; Ostelo 2003). However, the diversity of methods used for collection and reporting on healthcare utilisation meant that we were unable to undertake a meta-analysis on this outcome. Asenlof 2005 reported on healthcare utilisation in the two years following intervention as a dichotomous variable (counting any visit to a doctor, physiotherapist or other health professional as evidence of ongoing access to healthcare). No difference was observed between the structured goal setting and usual care groups for this measure

at two years (RR 1.32, 95\% CI 0.88 to 1.98). Cheng 2012 also reported no statistically significant difference between structured goal setting and usual care group in the number of emergency rooms visits, number of unplanned hospital readmissions, and length of hospital stays within 24 weeks. However, no means and SD were reported for these measures by Cheng 2012 for this measure. LaFerriere 1978 reported no difference between their structured goal setting and usual care group for the average number of therapy sessions each group received (MD 3.09 sessions, 95\% CI -0.66 to 6.84). Similarly, Oestergaard 2012 collected data on hours of occupational therapy received. Oestergaard $2012 \mathrm{did}$ not publish the means and SDs, but did report that there was no statistically significant difference between their structured goal setting and usual care groups in terms of occupational therapy hours provided. Ostelo 2003 completed a full economic analysis of the direct and indirect costs associated with healthcare and disability in the year following completion of their intervention. They too 
reported no difference between their structured goal setting and usual care groups in terms of total healthcare costs one year following intervention (MD EUR 639, 95\% CI -EUR 81.61 to EUR 1359.61).

\begin{abstract}
Adverse events
Three studies $(n=406)$ reported on the number of participants who withdrew from the studies due to adverse events: death (Cheng 2012; Parsons 2012); hospitalisation (Cheng 2012), or worsening symptoms (Ostelo 2003). When combined in a metaanalysis, there was no difference between structured goal setting and usual care groups in terms of the number of participants who withdrew from the studies due to adverse events of any cause (Peto Odd's Ratio 0.64 , 95\% CI 0.27 to 1.47; Analysis 2.5). However, given that this meta-analysis was based on raw data from two cluster-RCTs for which the effects of clustering could not be accounted (Parsons 2012; Cheng 2012), and given that the 95\% CI was wide even without adjusting for the effects of clustering, it was not possible to rule out differences in mortality between the two groups without considerably more data.
\end{abstract}

\section{Comparison 3: Interventions to enhance goal pursuit versus no interventions to enhance goal pursuit}

We identified two studies where the intervention and control groups differed solely in terms of the strategies used to enhance goal pursuit; the methods used for goal selection were identical for all participants (Culley 2010; Hart 2002). Both studies were at unclear risk of bias, both involved participants with traumatic brain injury, and both investigated the effectiveness of an intervention involving information technology (a Portable Voice Organizer in the case of Hart 2002; text messaging in the case of Culley 2010) to improve patient recall of rehabilitation goals. In both cases randomisation occurred at the level of the goals rather than at the level of participants. Each participant was required to have six goals for rehabilitation, either in order to enrol in the study (Culley 2010) or set as part of the study (Hart 2002). These goals were then randomised (three to each condition) to the intervention condition (to be communicated three times daily to participants via the information technology under investigation) or to the control group (no additional prompting about these goals was provided). Outcomes were measured in terms of the participants' recall of goals.

Two forms of recall were tested: open recall (where patients reported on their goals without any prompting) followed by cued recall (where patients were given pre-negotiated key words to help them remember their goals). Recall was scored on a 4-point scale from 0 (no recall) to 3 (complete recall) for each goal, resulting in a total score from 0 to 9 for each condition, for each participant. A full set of raw data for this outcome was published in Hart 2002 $(\mathrm{n}=10)$ and accessed via communication with the authors in the case of Culley $2010(\mathrm{n}=11)$.
We combined raw data on open recall from these two studies (n = 21) using an individual subject meta-analysis. In both cases, the data used in the meta-analysis were from the longest period of time following participant enrolment (one week for Hart 2002; two weeks for Culley 2010). We used a mixed linear model to estimate the differences between the intervention and control conditions for these two studies, and found a difference in favour of the use of information technology to facilitate the participants' open recall of rehabilitation goals (MD 3.1, 95\% CI 2.04 to 4.15; $\mathrm{P}<0.001$ ). No data were reported in these studies on health-related quality of life or self-reported emotional status, participation level outcomes, activity level outcomes, outcomes related to body structure or function, patient self-belief or engagement in rehabilitation, individual goal attainment, evaluation of care, service delivery level or adverse outcomes.

\section{Comparison 4: One structured approach to goal setting and/or strategies to enhance goal pursuit versus another structured approach to goal setting and/or strategies to enhance goal pursuit}

Nine trials compared different structured approaches to goal setting and/or strategies to enhance goal pursuit (see Included studies: Comparison groups).

\section{Collaboratively set versus therapist-mandated goal setting}

Bassett 1999 ( $\mathrm{n}=74 ; 11 \%$ attrition) compared outcomes when musculoskeletal physiotherapy goals were set collaboratively between the patient and treating physiotherapist versus when goals were prescribed by the physiotherapist without patient input. No differences between these two groups were reported for the number of treatment sessions required to achieve symptom relief (MD 2.15 sessions, $95 \% \mathrm{CI}-1.83$ to 6.13 ) or in the patients' self-reported home exercise adherence in terms of the percentage of prescribed sessions completed (MD 9.61\% of sessions, 95\% CI $3.45 \%$ to $22.67 \%$ ). Also reported was no difference between the two groups for self-reported symptom relief as measured on a 3point ordinal scale. However insufficient information was available to report relative risk arising from these data.

\section{Goal setting with operant conditioning versus goal setting without operant conditioning}

Both Blair 1991 ( $\mathrm{n}=89$; 11\% attrition) and Blair 1996 ( $\mathrm{n}=15$; no attrition) were conducted within nursing care homes. Both included two intervention groups where the approach to goal setting was identical (based on GAS methods), but where one of these two groups received additional operant conditioning interventions to enhance goal pursuit and the other did not. All goals focused on increasing the residents' independence in morning activities of daily living, with targeted activities selected as goals following collaborative discussion between the nursing staff and residents. 
The operant conditioning interventions included prompting (i.e. reminders to do target activities), shaping (i.e. helping with set up of activities), and reinforcement. Reinforcement included smiles, praise, affectionate touches, and conversation when residents completed or partly completed the target activities, and a withdrawing of these responses when residents refused to do a task for no clear reason. After six weeks of nursing intervention and at a 22-week follow-up, GAS scores were collected for participants in both Blair 1991 and Blair 1996. However, in both these studies GAS measures were treated as interval data (reported as means and SDs) rather than ordinal data (as required by our protocol; see Measures of treatment effect), so no results could be extracted for the purposes of this review. In addition to GAS scores, Blair 1991 reported no difference between the two goal setting groups on the basis of Rosenburg Self-Esteem scores at the 22-week follow-up (MD $0.39,95 \%$ CI -0.89 to 0.11 ; results unadjusted for clustering effects as no intraclass correlations for this measure were available).

\section{Setting an end goal only versus setting incremental short- term steps towards an end goal}

Conrad 2000 reported on a small RCT ( $\mathrm{n}=7$; no attrition) where patients with coronary artery disease were randomised to receive either an immediate goal of decreasing their dietary fat to $10 \%$ of total energy consumption or a series of short-terms goals gradually reducing their dietary fat to $10 \%$ of total energy consumption over a four-month period. After seven months, no differences were observed between the two groups on the basis of self-reported dietary fat consumption, cholesterol consumption, protein consumption, body weight, or serum cholesterol. For self-reported dietary fat consumption as a percentage of total energy consumption (the primary outcome in the hypothesis for testing) the difference in means after four months was $7.0 \%$ (95\% CI $-11.6 \%$ to $25.6 \%)$.

\section{Setting a specific goal versus setting a non-specific goal}

James 1993 ( $\mathrm{n}=37$ in the two cognitive behaviour therapy groups; $30 \%$ attrition) recruited patients participating in cognitive behavioural therapy for chronic headache, and randomised patients to receive either a specific, time-limited goal to practice pain-coping strategies for a prescribed duration and frequency on a daily basis over a six-week period or the non-specific goal of practicing pain-coping strategies 'as much as possible for as long as possible' (p. 310) over the six-week period. Multiple measures were used, resulting in 21 different outcome variables that James 1993 tested for significance (one for pain severity, six for medication use, one for self-efficacy, one for activity levels, four for pain behaviour, one for pain-related disability, four for psychological states and traits, and three for coping). No differences between the specific and non-specific goal groups were found after therapy except for three of the four pain behaviour measures, which all favoured the specific goal group. The pain behaviour measure with the median effect size was for the nonverbal complaints subscore on the Pain Behavior Questionnaire (MD -3.02, 95\% CI -5.83 to -0.21).

\section{Goal Management Training versus Identity Oriented Goal Training}

McPherson 2009 described a pilot study ( $\mathrm{n}=34 ; 35 \%$ attrition) in which participants with traumatic brain injury were randomised to one of three groups: 1) Goal Management Training, 2) Identity Oriented Goal Training, or 3) usual care. The two goal setting approaches were based on self-regulation theory and designed to address impairments in self-regulation secondary to brain injury. Goal Management Training involved identification and documentation of a rehabilitation goal and the development and rehearsal of planned steps to achieve that goal, with an emphasis on errorless learning (i.e. avoidance of goal failure). Identity Oriented Goal Training involved development of an 'identity map' to use as a tool for identification and articulation of goals that would help participants connect with and progress towards a meaningful, higherorder life goal. Outcomes from GAS scores were evaluated after eight weeks of therapy and at a three-month follow-up assessment. Based on raw data from each participant (accessed via author communication) three out of eight participants in Goal Management Training and four out of five participants in the Identity Oriented Goal Training groups achieved or exceeded at least one of their set goals (with each participant having between one and three goals), with no difference observed between the two groups in terms of goal attainment (RR $0.47,95 \%$ CI 0.17 to 1.27 ).

\section{Setting a difficult goal versus setting an easier goal}

In Miller 2012 ( $\mathrm{n}=46 ; 24 \%$ attrition) people with type 2 diabetes participated in a 5-week dietary intervention aimed at increasing their consumption of lower glycaemic index foods. Participants were randomised to receive a specific, difficult goal (to change their dietary behaviour to include eight servings per day of lower glycaemic index foods) or a specific, easier goal (to change their dietary behaviour to include six servings per day of lower glycaemic index foods). After eight weeks both groups had increased their consumption of lower glycaemic index foods from baseline (based on four-day self-report) and there was no significant difference between the two groups in terms of total servings per day of lower glycaemic index foods (MD - 0.02 servings, $95 \%$ CI -0.63 to 0.59 ). Participants in the group receiving the specific difficult goal were less committed to their treatment goal, as measured on a 5-point scale (MD $0.41,95 \%$ CI 0.32 to 0.50 ), but there was no reported difference between the two groups for the participants' level of satisfaction or dissatisfaction with meeting their goal, as measured on a 9-point scale (MD 0.33, 95\% CI 0.07 to 0.59). Furthermore, there was no difference between the two groups in terms of taskspecific self-efficacy (MD $0.03,95 \%$ CI -0.17 to 0.23 ). 
Setting a high intensity exercise goal versus setting a goal of non-specific exercise intensity

Richardson 2007 ( $\mathrm{n}=35$; 14\% attrition) investigated the effect of different types of exercise goals for people with type 2 diabetes participating in a six-week home-based exercise programme delivered via the Internet with the use of a pedometer to monitor exercise duration and intensity. The participants were randomised to either a 'lifestyle goal' or a 'structured goal'. The lifestyle goalcentred on increasing total daily step counts regardless of exercise intensity (a goal of non-specific exercise intensity); the structured goal involved increasing total daily step counts for high-intensity exercise only (a high-intensity exercise goal). High-intensity exercise was defined as exercise that occurred for a minimum of ten minutes at a time, with at least 60 steps per minute. Both lifestyle goals and structured goals were automatically assigned and altered weekly by the Internet interface based on the previous week's step count data (uploaded via the pedometer; starting with one week of baseline recording). Lifestyle goals were automatically set at 1200 steps more per day than the previous weeks' average daily step count, up to a maximum of 10,000 steps per day. Structured goals were automatically set at 800 steps more per day of high-intensity exercise only, compared to the previous weeks' average daily step count for high-intensity exercise, up to a maximum of 10,000 steps per day. At the end of the study, there was no difference between the two groups in terms of average total daily step counts (MD 589 steps, $95 \%$ CI -1985 to 3163 steps) or average total daily step counts involving high-intensity exercise only (MD 546 steps, $95 \%$ CI -1442 to 2534 ). However, the participants in the structured goal group were found to be less satisfied with the exercise programme. Only $62 \%(8 / 13)$ of the structured goal group were inclined to definitely recommend the programme to a friend in comparison to $100 \%(17 / 17)$ of the lifestyle goal group (RR 0.62 , $95 \%$ CI 0.41 to 0.96 ), and only $31 \%$ (4/13) of the structured goal group reported finding the programme 'very useful' compared to
$71 \%(12 / 17)$ of the lifestyle goal group (RR $0.44,95 \%$ CI 0.18 to 1.04$)$. The lifestyle goal group also wore their pedometers for more hours each day than did the structured goal group (MD 2 hours, $95 \%$ CI 0.2 to 3.8 hours).

\section{High level versus low level patient involvement in goal setting}

In Webb 1994 ( $\mathrm{n}=16$; no attrition) people participating in a day hospital or residential rehabilitation programme for traumatic brain injury were randomised to either a high level or a low level of involvement in goal setting. Both groups of participants were formally oriented to goal setting in rehabilitation at the beginning of the study and both were involved in prioritisation of their rehabilitation goals, with therapists converting the participants' chosen goals into a GAS scale to be achieved over the following eight weeks. The two groups differed in that the high involvement group also participated in a discussion of the importance of goal setting and were encouraged to ask questions about goal setting at the beginning of rehabilitation, whereas the low involvement group were not. Furthermore, the high involvement group had options for rehabilitation goals presented to them as possible goals written on wooden blocks that the participants could use to help order and communicate their preferences. In comparison, the low involvement group selected goals from a list written on paper. Finally, the high involvement group were encouraged to discuss their goals on a weekly basis for the duration of the study, with progress towards goals regularly documented by and for the participants on worksheets and in a goal diary. Outcomes on the basis of GAS scores were evaluated after eight weeks of rehabilitation. However, as GAS measures were treated as interval data (reported as means and SDs) rather than ordinal data (as required by our protocol; see Measures of treatment effect), no results could be extracted for the purposes of this review. 
ADDITIONAL SUMMARY OF FINDINGS[Explanation]

Structured goal setting with or without strategies to enhance goal pursuit compared to 'usual care' that involved some goal setting but where no structured approach was followed for adults with acquired disabilityparticipating in rehabilitation

Patient or population: adults with acquired disability participating in rehabilitation

Settings: inpatient, outpatient, and community-based healthcare services

Intervention: structured goal setting with or without strategies to enhance goal pursuit

Comparison: 'usual care' that involved some goal setting but where no structured approach was followed

\begin{tabular}{|c|c|c|c|c|c|}
\hline \multirow[t]{3}{*}{ Outcomes } & \multicolumn{2}{|c|}{ Illustrative comparative risks* $(95 \%$ Cl) } & \multirow{3}{*}{$\begin{array}{l}\text { No of Participants } \\
\text { (studies) }\end{array}$} & \multirow{3}{*}{$\begin{array}{l}\text { Quality of the evidence } \\
\text { (GRADE) }\end{array}$} & \multirow[t]{3}{*}{ Comments } \\
\hline & Assumed risk & Corresponding risk & & & \\
\hline & 'Usual care' & $\begin{array}{l}\text { Structured goal setting (with } \\
\text { or without strategiesto en- } \\
\text { hance goal pursuit) }\end{array}$ & & & \\
\hline $\begin{array}{l}\text { Health-related quality of life } \\
\text { or self-reported emotional } \\
\text { status } \\
\text { Follow-up: median } 24 \text { weeks }\end{array}$ & $\begin{array}{l}\text { The mean Mental Component } \\
\text { Summary Scores on the Short } \\
\text { Form-36 for the control group } \\
\text { was } \\
\mathbf{5 8 . 5} \text { points (SD 10.0) (out of } \\
\text { a possible score of } 0-100)^{1}\end{array}$ & 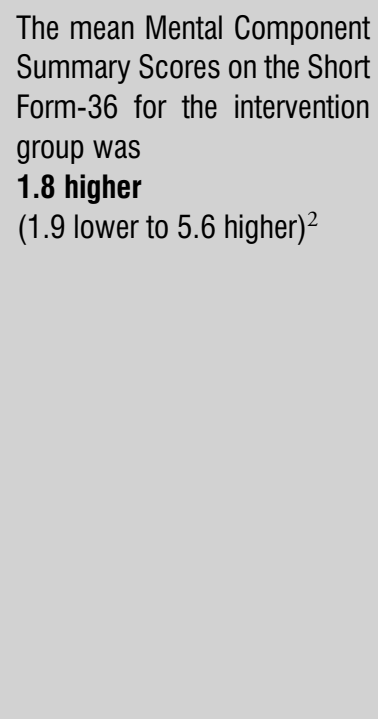 & $\begin{array}{l}441 \\
\text { (5 studies) }\end{array}$ & $\begin{array}{l}\oplus \oplus \bigcirc \bigcirc \\
\text { low }^{3,4}\end{array}$ & $\begin{array}{l}\text { Higher scores indicate better } \\
\text { outcomes. Scores estimated } \\
\text { using a SMD of } 0.18 \text { (95\% } \\
\mathrm{Cl}-0.19 \text { to } 0.56 \text { ). One addi- } \\
\text { tional quasi-RCT with } 201 \text { par- } \\
\text { ticipants reported no means or } \\
\text { SD, but indicated that usual } \\
\text { care may lead to higher qual- } \\
\text { ity of life than structured goal } \\
\text { setting. One further study with } \\
122 \text { participants reported that } \\
\text { participants in the structured } \\
\text { goal setting group were more } \\
\text { likely to report being more sat- } \\
\text { isfied or much more satisfied } \\
\text { with their daily life compared } \\
\text { to participants in the usual care } \\
\text { group } 3 \text { months post interven-- }\end{array}$ \\
\hline
\end{tabular}




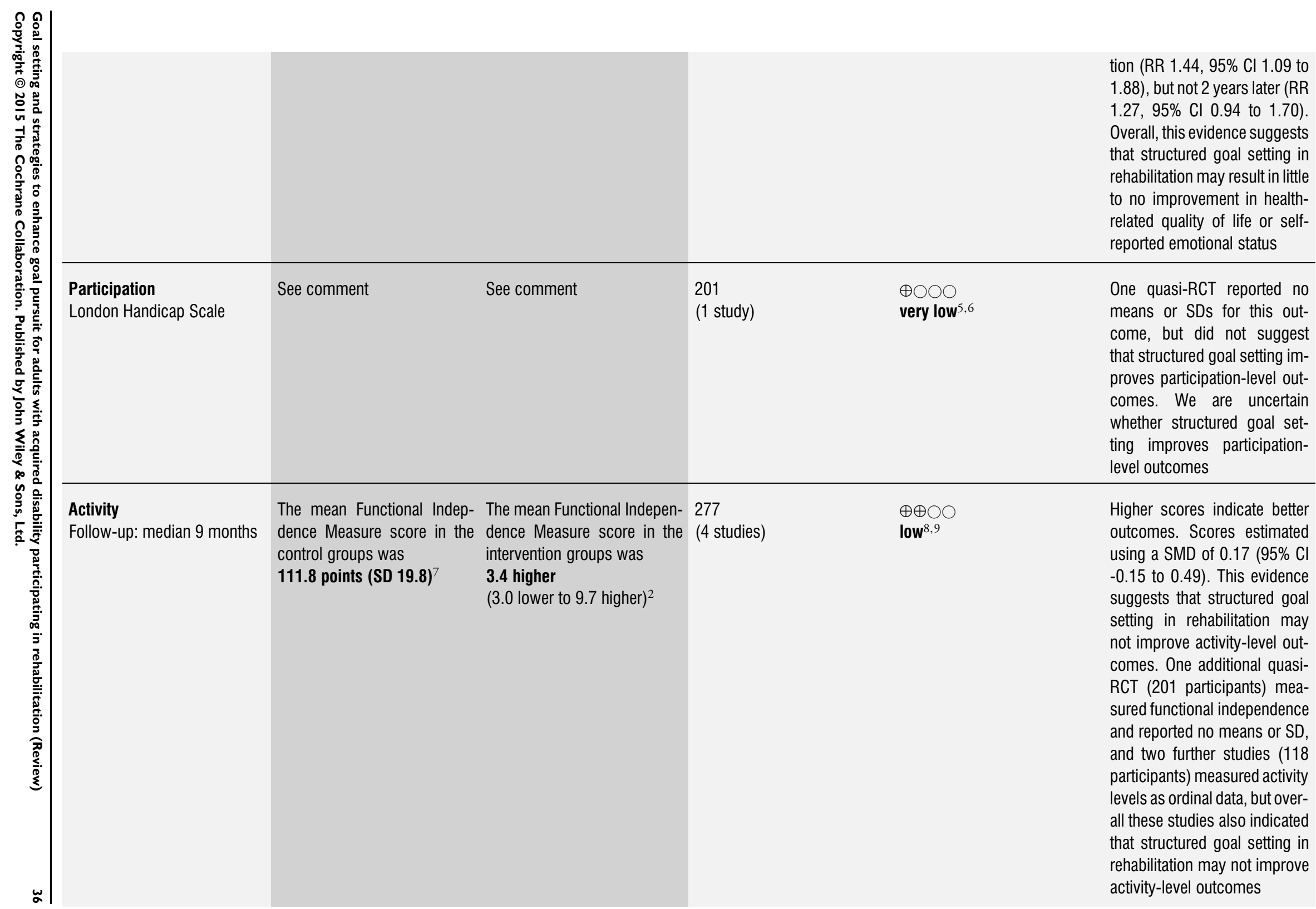




\begin{tabular}{|c|c|c|c|c|c|}
\hline $\begin{array}{l}\text { Body structure and body } \\
\text { function } \\
\text { Follow-up: median } 15 \text { months }\end{array}$ & See comment & See comment & $\begin{array}{l}229 \\
\text { (3 studies) }\end{array}$ & $\begin{array}{l}\oplus \bigcirc \bigcirc \bigcirc \\
\text { very low }\end{array}$ & $\begin{array}{l}\text { Unable to pool outcomes due } \\
\text { to lack of similarities in the } \\
\text { types of measures used. We } \\
\text { are uncertain whether struc- } \\
\text { tured goal setting improves } \\
\text { outcomes at the level of body } \\
\text { structure and body function }\end{array}$ \\
\hline $\begin{array}{l}\text { Engagement in rehabilitation } \\
\text { Follow-up: median } 5 \text { weeks }\end{array}$ & See comment & See comment & $\begin{array}{l}32 \\
\text { (1 study) }\end{array}$ & $\begin{array}{l}\oplus \bigcirc \bigcirc \bigcirc \\
\text { very low } 5,9\end{array}$ & $\begin{array}{l}\text { One study reported data on } \\
\text { patient motivation in rehabil- } \\
\text { itation. A small difference in } \\
\text { favour of structured goal set- } \\
\text { ting in comparison to usual } \\
\text { care was reported in terms of } \\
\text { patient-rated motivation (MD } \\
1.40 \text { on a } 10 \text {-point scale of } \\
\text { self-reported motivation, } 95 \% \\
\text { Cl } 0.43 \text { to } 2.37 \text { ) but not for } \\
\text { therapist-rated score of moti- } \\
\text { vation (MD } 0.48 \text { on an 8-point } \\
\text { scale of therapist-rated patient } \\
\text { motivation, } 95 \% \mathrm{Cl}-0.41 \text { to } 1 \text {. } \\
37 \text { ) }\end{array}$ \\
\hline $\begin{array}{l}\text { Self-efficacy } \\
\text { Follow-up: } 18 \text { months }\end{array}$ & $\begin{array}{l}\text { The mean self-efficacy in the } \\
\text { control groups was } \\
\mathbf{1 6 8 . 6} \text { points (SD 29.8) (on a } \\
\text { scale of } 0 \text { to 200) }\end{array}$ & $\begin{array}{l}\text { The mean self-efficacy in the } \\
\text { intervention groups was } \\
\mathbf{1 1 . 0} \text { higher } \\
(0.6 \text { to } 21.2 \text { higher })^{2}\end{array}$ & $\begin{array}{l}134 \\
\text { (2 studies) }\end{array}$ & $\begin{array}{l}\oplus \bigcirc \bigcirc \bigcirc \\
\text { very low }\end{array}$ & $\begin{array}{l}\text { Higher scores indicate bet- } \\
\text { ter self-efficacy. Scores esti- } \\
\text { mated using a SMD of } 0.37 \\
(95 \% \mathrm{Cl} 0.02 \text { to } 0.71) \text {, indica- } \\
\text { tive of an effect size that may } \\
\text { range from small to large }\end{array}$ \\
\hline
\end{tabular}

${ }^{*}$ The basis for the assumed risk (e.g. the median control group risk across studies) is provided in footnotes. The corresponding risk (and its $95 \%$ confidence interval) is based on the assumed risk in the comparison group and the relative effect of the intervention (and its $95 \% \mathrm{Cl}$ ).

Cl: Confidence interval; RR: Risk ratio; MD: mean difference; RCT: randomised controlled trial; SD: standard deviation; SMD: standard mean difference 
GRADE Working Group grades of evidence

High quality: Further research is very unlikely to change our confidence in the estimate of effect.

Moderate quality: Further research is likely to have an important impact on our confidence in the estimate of effect and may change the estimate.

Low quality: Further research is very likely to have an important impact on our confidence in the estimate of effect and is likely to change the estimate.

Very low quality: We are very uncertain about the estimate.

${ }^{1}$ The Mental Component Summary Score on the Short Form-36 was used for this illustrative comparative risk as this was deemed to be the most common, most general measure of quality of life used in the studies included in the meta-analysis for this outcome. The data on assumed risk for the Mental Component Summary Score on the Short Form-36 was taken from control group data in the study that used this measure (Parsons 2012).

${ }^{2}$ The difference in the corresponding risk (and its $95 \% \mathrm{Cl}$ ) was calculated by multiplying the SD for the assumed risk by the SMD from the meta-analysis (and its $95 \% \mathrm{Cl}$ ).

${ }^{3}$ The GRADE rating was downgraded due to the presence of substantial unexplained heterogeneity in the data.

${ }^{4}$ The GRADE rating was downgraded due to the $95 \%$ confidence interval crossing the line of no effect as well as reaching above an SMD of 0.5 .

${ }^{5}$ The GRADE rating was downgraded by two levels due to high risk of bias.

${ }^{6}$ The GRADE rating was downgraded due to there being no published information on effect size or variance

7 The Functional Independence Measure was used for this illustrative comparative risk as this was deemed to be the most common, most general measure of activity levels used in the studies included in the meta-analysis for this outcome. The data on assumed risk for the Functional Independence Measure was taken from control group data in the study that used this measure (Taylor 2012).

${ }^{8}$ The GRADE rating was downgraded by one level, given overall unclear risk of bias.

${ }^{9}$ The GRADE rating was downgraded due to the small number of participants and high attrition rate.

${ }^{10}$ The GRADE rating was downgraded due to the findings being based on descriptive analysis of a series of small studies that could not be pooled in a meta-analysis, reaching different conclusions regarding treatment effect,

${ }^{11}$ The Self-efficacy Scale was used for this illustrative comparative risk as this was deemed to be the most common, most general measure of self-efficacy used in the studies included in the meta-analysis for this outcome. The data on assumed risk for the Selfefficacy Scale was taken from control group data in the study that used this measure (Asenlof 2005). 


\section{DISCUSSION}

\section{Summary of main results}

The results from data on the effects of goal setting interventions were divided into four sections. The first of these comprised studies where, within the context of a rehabilitation intervention, any type of goal setting (with or without strategies to enhance goal pursuit) was compared to the same rehabilitation intervention provided without goal setting.

Based on the GRADE assessment (Summary of findings for the main comparison), there is very low quality evidence that goal setting results in a moderate increase in health-related quality of life or self-reported emotional status. There is also very low quality evidence that goal setting results in a large increase in patient self-efficacy. However, we found no evidence of an effect of goal setting (with or without strategies to enhance goal pursuit) on activity, improvements in body structure or body function, or levels of engagement in rehabilitation, although these conclusions were based on a small number of studies with an overall moderate risk of bias. There is insufficient data to draw conclusions regarding the possibility of adverse events arising from goal setting interventions.

The second section of our results comprised studies where, within the context of a rehabilitation intervention, a structured approach to goal setting (with or without strategies to enhance goal pursuit) was compared to 'usual practice' in which some goals were set, but no structured or required approach to goal setting was followed. Conclusions from these studies are more difficult to reach as most studies were not explicit regarding what goal setting in usual care typically involved. This makes it difficult to be certain how the structured goal setting interventions that the experimental groups received differed from the process of goal setting provided to participants in the control groups. However, broadly speaking, the structured goal setting interventions appeared to involve more patient participation in goal selection, were more person-centred, and focused more on personally meaningful outcomes that patients wanted to achieve, with some studies also including additional strategies to enhance patient behaviour directly related to goal pursuit.

Based on the GRADE assessment (Summary of findings 2), there is very low quality evidence that the more structured approaches to goal setting result in a small to moderate increase in patient self-efficacy. There is also low quality evidence that patients are more satisfied with service delivery if receiving a more structured approach to goal setting in comparison to usual care. However, no evidence was found for the effect of structured goal setting in comparison to usual care with regard to health-related quality of life, patient-reported emotional status, or activity levels, although these conclusions are based on a small number of studies with an overall moderate risk of bias. Insufficient information exists to draw conclusions regarding the effects of structured goals on outcomes at the level of social participation or patient engagement in rehabilitation, or regarding the risk of adverse events arising from structured goal setting.

The third section of our results included studies where, within the context of a rehabilitation intervention, one approach to enhancing goal pursuit was compared to another. We identified only two studies in this group of trials, both of which investigated the same strategy to enhance goal pursuit (i.e. use of information technology to help patients recall their goals). These studies were at unclear risk of bias but involved a total of only 21 participants. When individual patient data from these studies were pooled there was low quality evidence that using information technology (text messaging or portable voice organisers) improved the ability of people with brain injury to recall their goals for rehabilitation.

The final section of our results contained studies where one structured approach to goal setting (with or without strategies to enhance goal pursuit) was compared to another structured approach. Each of the studies in this section involved testing a different intervention regarding goal setting or goal pursuit (e.g. goal specificity, goal difficulty, interventions to address impairment of selfregulation related to goal pursuit, and so forth), with outcomes being evaluated through a wide range of measures. All results for this group of studies are based on single trials, with small sample sizes, and are at an overall moderate risk of bias. As a consequence, there is insufficient information overall to draw any firm conclusions regarding the effects of various structured approaches to goal setting and goal pursuit on health outcomes.

\section{Overall completeness and applicability of evidence}

This review is based on a comprehensive search strategy without restrictions on language or publication status. The definitions we used for 'acquired disability' and 'rehabilitation' when making decisions about whether or not to include studies in this review were broad, so erred on the side of inclusiveness in terms of clinical context. Included studies involved participants with a wide range of disabling health conditions, receiving rehabilitation in inpatient through to community settings, from countries in North America, Europe, and Australasia. Only one study involved participants from Asia (specifically, Hong Kong). The scope of the review could have potentially been further broadened to also include healthcare interventions for people with disability that had been acquired in childhood (such as intellectual disability or congenital disorders), however this would likely have just resulted in new problems regarding what to set as boundaries for inclusion and exclusion of studies.

Convincing, high quality evidence on the effectiveness of goal setting interventions was lacking, so answers to the review questions remain incomplete or uncertain. Furthermore, the individual meta-analyses we conducted for the primary outcomes of interest were each based on few studies, with the largest meta-analysis involving nine studies. This meant that while we were able 
to reach some conclusions regarding our broad research question (e.g. Is any type of goal setting better than no goal setting?), we were unable to undertake subgroup analysis to examine the contribution of specific aspects of goal setting processes to the overall effects of goal setting on clinical outcome or to explore reasons for heterogeneity among studies reporting on similar types of outcomes. The following is a list of the types of questions that we were unable to address in this review, having insufficient studies on which to conduct subgroup analyses.

- Do higher levels of patient involvement in goal setting or goal planning result in better clinical outcomes?

- Do higher levels of family/carer involvement in goal setting or goal planning result in better clinical outcomes?

- Do goals that focus on activity or participation domains, as defined by the ICF (WHO 2001a) result in better clinical outcomes in comparison to goals that focus on objectives at the level of body structure and function?

- Do difficult, ambitious, or challenging goals result in better clinical outcomes in comparison to easily achievable goals?

- Does the use of written or oral feedback to patients regarding progress towards goals result in better clinical outcomes?

Furthermore, there are very few studies that consider the cost of goal setting. We identified only one study which included a complete economic evaluation of the cost and outcomes of a structured approach to goal setting in comparison to usual care (Ostelo 2003). Goal setting often requires the input of additional time from health professionals, and the cost of this can multiply when teams of health professionals are involved in goal setting meetings, so studies that investigate whether the added benefits of goal setting (if these do indeed exist) merit the additional cost accrued are well worth undertaking.

\section{Quality of the evidence}

'Risk of bias' ratings (see Figure 1) show the variability across all studies. The criterion for which studies scored worst was the blinding of participants and personnel. This was not surprising given the nature of the interventions under investigation, which most often required active involvement of patients and their treating health professionals in order to be implemented. A small number of studies addressed (or partly addressed) the blinding of participants and personnel by automating the delivery of goal setting via information technology or by having a third party set goals with the patients and not informing the treating health professionals of these goals. However these approaches limit the generalisability of findings to only certain types of clinical contexts. In future trials, lack of blinding of participants and personnel will necessarily remain a limitation of studies in this area of clinical practice.

The next two criteria for which studies scored worst were the blinding of outcome assessment and incomplete collection of data, with 27 of the 39 included studies being at high risk of bias on one or both of these criteria. Unlike blinding of participants and personnel there is no reasonable methodological explanation for not blinding outcome assessors to group allocation. This is an issue that should be addressed in all future RCTs on rehabilitation goal setting. Incomplete data collection resulting from attrition of participants presents more of a challenge for rehabilitation studies, particularly when data are to be collected over a long period of time, and particularly for certain clinical populations with higher rates of itinerant lifestyles or mortality. However, researchers should consider and include strategies to maximise retention of participants in RCTs once recruited, even if only for outcome assessment, and should include intention-to-treat analyses within their study design.

For the main findings reported in this review, we downgraded the quality of evidence due to either overall unclear or high risk of bias, unexplained heterogeneity, wide confidence intervals, or small sample sizes. This means that overall we are uncertain about the outcome estimates reported, and further research is very likely to have an important impact on our confidence in the estimates of effect, including being likely to change these estimates.

\section{Potential biases in the review process}

The methods and searches for this review were rigorous and should be relatively low in bias. We made only minor amendments to our protocol (Levack 2012; see Differences between protocol and review) which should have had little impact on potential bias. The most significant of these amendments was the decision, after completing data extraction but prior to data analysis, to combine measures of self-reported emotional status with measures of healthrelated quality of life. Our justification for this was that insufficient studies reported data using measures of health-related quality of life and that the two concepts were deemed to be sufficiently similar for the results of a meta-analysis to be clinically meaningful. However, as this was a post-hoc decision, the risk of bias arising from this aspect of the review process should be considered slightly higher than if these decisions had been made during the protocol stage. One further consideration is that authors of this review were also authors of two of the included studies (McPherson 2009; Taylor 2012). Adherence to our published protocol however minimised risk of bias arising from this, and as both were pilot studies they have had little influence on the overall conclusions of this review.

\section{Agreements and disagreements with other studies or reviews}

To our knowledge there are three prior systematic reviews of goal setting in rehabilitation: two specifically investigating goal setting in stroke rehabilitation (Rosewilliam 2011; Sugavanam 2013) and one investigating goal setting in all rehabilitation literature (Levack 
2006a). Based on appraisal of observational studies and qualitative research, the two reviews of goal setting within the context of stroke rehabilitation concluded that active patient participation in goal setting appeared to be something that patients value and that structured methods of goal setting seem to increase patients' perceptions of their level of involvement in clinical decision-making (i.e. enhancing a sense of self-determination) (Rosewilliam 2011; Sugavanam 2013). The third review concluded that some experimental studies had provided limited evidence that goal setting might increase patient adherence to treatment regimens (Levack 2006a). Overall however, these three systematic reviews concluded that there was insufficient experimental research of adequate quality to allow any firm conclusions to be drawn regarding what effect, if any, specific goal setting practices have on health outcomes following rehabilitation.

Our current review differs from these past reviews in four main ways. It has involved: 1) a published protocol with an a priori plan for analysis (Levack 2012); 2) a far more comprehensive search of the literature including the screening of over 9000 titles and the inclusion of non-English and grey literature, research theses, and conference presentations; 3) categorisation of studies on the basis of comparison types, for which a particularly important distinction has been made between studies with no goal setting as a control group and studies with usual care goal setting as a control group; and 4 ) pooling of the main outcomes where possible with metaanalyses.

Our review concurs with past reviews regarding the limited quality and quantity of experimental studies on goal setting in rehabilitation, although our latest review has resulted in the identification of substantially more RCTs. For instance, Levack 2006a identified 13 RCTs while in this latest review we have identified 33 RCTs and six additional quasi-RCTs. Some of this increase in the number of trials is due to increased interest since 2006 in goal setting in rehabilitation as a research topic, but it is also the result of a more comprehensive search strategy, which also identified more relevant studies published prior to 2006 .

This review, while still limited by the quality of evidence and diversity of studies underpinning it, provides a more robust and transparent evaluation of the evidence than past reviews. We have found some (very low quality) evidence of the value of any type of goal setting, in comparison to no goal setting, in terms of a potentially positive impact on health-related quality of life or patientreported emotional status and in terms of higher patient self-efficacy. We are more equivocal regarding the impact of goal setting versus no goal setting, or structured approaches to goal setting versus usual care, on patient engagement in rehabilitation than in our previous review (Levack 2006a). Evidence regarding the individual contribution of specific components of the goal setting process (e.g. levels of patient involvement, levels of goal difficulty) remains inconclusive.

\section{A U THORS'CONCLUSIONS}

\section{Implications for practice}

Based on the GRADE assessment, there is very low quality evidence that including any type of goal setting in rehabilitation practice for adults with acquired disability is better than no goal setting in terms of patient-reported health-related quality of life or emotional status and self-efficacy. There is also very low quality evidence that more structured approaches to goal setting result in higher patient self-efficacy and satisfaction with service delivery in comparison to usual care where goal setting follows a less formal structure. The evidence is inconclusive, however, regarding whether or not goal setting results in improvements in social participation, levels of activity, amelioration of impairments of body structure or body function, or a higher level of patient engagement in the rehabilitation process.

Although it is not clear which components of current goal setting practice produce these effects, structured approaches to goal setting in these studies tended to be characterised by higher levels of patient participation in goal selection, greater emphasis on personcentredness, a greater focus on personally-meaningful outcomes that patients want to achieve, and attention to behaviour change strategies intended to positively influence goal pursuit. We could speculate that the emphasis on person-centredness and personally-meaningful goals has favoured outcomes related to subjective rating of quality of life, feeling of personal control (self-efficacy), and high satisfaction with service delivery, but has not favoured outcomes related to physical performance of activities or objective measures of social participation. It is possible that different approaches to goal setting other than those examined in the studies included in this review could potentially produce different effects that are more beneficial in terms of these objective measures of improved health outcomes. As just one example, a goal setting approach based on Locke and Latham's goal theory (Locke 2002) would place greater emphasis on selecting goals that maximise attention and effort during therapeutic activities rather than on linking goals to personally-meaningful outcomes, and therefore could potentially produce different results in terms of physical outcomes following rehabilitation. We note that while research on this approach to goal setting is often cited in rehabilitation literature (Siegert 2014a) it has seldom been studied in clinical rehabilitation trials, as demonstrated by the findings from this review. Further research is required however, to test the hypothesised effects of different approaches to goal setting for different people in different clinical contexts.

One important caveat to this review is that goal setting can serve multiple functions in rehabilitation; improving patient outcomes on standardised outcome measures is only one such function. Other reasons might include improving team cohesion, measuring individualised rehabilitation outcomes, or enhancing accountability to a funder of health services (Levack 2006b; Levack 2006c). 
Health professionals also have a moral obligation to involve patients, and at times their families, in decision-making about the objectives of therapy, and thus may undertake person-centred or family-centred goal setting to meet this obligation. Whether or not goal setting provides the best forum for fulfilling these moral obligations is, however, a philosophical issue, and one which is not going to be answered through the implementation of quantitative methods. For further discussion on ethics and goal setting, see Levack 2014b.

\section{Implications for research}

All future research should view goal setting as a complex health intervention, and so should follow established guidelines for experimental research on complex interventions (e.g. Craig 2008). This includes the articulation of a well-substantiated theory about how goal setting might achieve its hypothesised effects (there currently exist a number of such theories) and a close alignment between a chosen theory and the methods used to implement and test goal setting in a clinical trial. Methods for future studies need to include a comprehensive description of the approaches to goal setting provided to both the experimental and control groups. This should include (but not be limited to) any training or preparation of the patient and their family for involvement in goal setting, the complete process of goal selection, what restrictions are placed around the content and format of rehabilitation goals, and information on how goals are then used to influence patient behaviour, professional behaviour, or clinical practice. Without such descriptions, comparison between clinical trials will continue to be limited.

The use of established tools such as intervention mapping (Bartholomew 1998; Kok 2004) and process evaluation (Moore 2015) may assist with the development and documentation of goal setting approaches in training manuals for the health professionals who are taking part in the research, to facilitate higher treatment fidelity, to permit replication of studies, and to ultimately assist with the translation of research to practice, should an approach to goal setting prove effective.

Further research is required to strengthen our confidence in the direction and size of effects associated with goal setting in rehabilitation and to specify the individual contribution of components of goal setting practice to improve rehabilitation outcomes where these might exist. In particular, research studies should be designed to examine the effects of higher levels versus lower levels of patient and/or family involvement in goal setting; highly difficult or highly challenging versus easily achievable goals or goals that progress in small steps; written and/or oral feedback to patients about progress towards goal versus no feedback; and the effect of different types of goal targets (e.g. at the level of life goals, participation, activity, or body structure and function) on health outcomes. The cost and time required to deliver different approaches to goal setting should also be considered in future research. Furthermore, no experimental research has yet been conducted on the effects of goal setting on health professional behaviour (such as motivation, teamwork practices, and intensity of service delivery) or the risk of adverse events arising from different approaches to goal setting. These too could be useful foci for future research.

It seems likely that goal setting practice will have different effects for different people in different clinical contexts. As such, a larger pool of goal setting studies will make it increasingly possible to undertake subgroup analyses to examine the specific effects of goal setting in particular rehabilitation environments. However, future research in rehabilitation could also begin to examine patients' cultural, psychological, and personality characteristics to see if certain types of goal setting are more or less effective for different types of people.

In addition, all future research on goal setting in rehabilitation should strive to address common problems with study design that result in higher risk of bias, e.g. concealment of group allocation, blinding of outcome assessments, minimisation of attrition, and attention to intention-to-treat analysis. As blinding of therapists and patients to group allocation is frequently not possible in goal setting studies, development of strategies to enhance fidelity to intervention protocol is important (Poltawski 2014). For further discussion of challenges and recommendations associated with the implementation of clinical trials on goal setting in rehabilitation, see Levack 2014c.

\section{ACKNOWLEDGEMENTS}

We thank the staff and editors of the Cochrane Consumers and Communication Review Group, especially Sophie Hill, Megan Prictor, Rebecca Ryan, and Sue Cole, for their prompt and helpful advice and assistance. We would also like to thank John Kis-Rigo, Trials Search Co-ordinator, Cochrane Consumers and Communication Group for compiling the MEDLINE search strategy. We also greatly appreciated the time put into this review by the peer reviewers: Kate Laver, Claire Glenton, Paul Tobin, Derick Wade, and Nancy Santesso, and by Vicki Rowland, who proofread our final review draft. Finally, we would like to most sincerely thank all the researchers who contribute their time to answering our questions regarding their studies and providing additional data from these studies when it was requested. 


\section{R E F E R E N C E S}

\section{References to studies included in this review}

\section{Arnetz 2004 \{published data only\}}

Arnetz JE, Almin I, Bergstrom K, Franzen Y, Nilsson H. Active patient involvement in the establishment of physical therapy goals: effects on treatment outcome and quality of care. Advances in Physiotherapy 2004;6(2):50-69.

Asenlof 2005 \{published data only\}

Åsenlöf P, Denison E, Lindberg P. Idiographic outcome analysis of the clinical significance of two interventions for patients with musculoskeletal pain. Behavior Research and Therapy 2006;44:947-65.

* Åsenlöf P, Denison E, Lindberg P. Individually tailored treatment targeting activity, motor behavior, and cognition reduces pain-related disability: a randomized controlled trial in patients with musculoskeletal pain. Journal of Pain 2005;6(9):588-603.

Åsenlöf P, Denison E, Lindberg P. Long-term follow-up of tailored behavioural treatment and exercise based physical therapy in persistent musculoskeletal pain: a randomized controlled trial in primary care. European Journal of Pain 2009;13:1080-8.

Bassett 1999 \{published data only\}

Bassett SF, Petrie KJ. The effect of treatment goals on patient compliance with physiotherapy exercise programmes. Physiotherapy 1999;85(3):130-7.

Bell 2003 \{published data only\} Bell M, Lysaker P, Bryson G. A behavioral intervention to improve work performance in schizophrenia: work behavior inventory feedback. Journal of Vocational Rehabilitation 2003;18(1):43-50.

Blair 1991 \{published data only\}

* Blair CE. Effect of Mutual Goal Setting and Behavior Modification Techniques on Self-Care Behaviors of Nursing Home Residents [PhD thesis]. Austin, USA: University of Texas, 1991.

Blair CE. Combining behavior management and mutual goal setting to reduce physical dependency in nursing home residents. Nursing Research 1995;44:160-5.

Blair 1996 \{published data only\}

Blair CE, Lewis R, Vieweg V, Tucker R. Group and singlesubject evaluation of a programme to promote self-care in elderly nursing home residents. Journal of Advanced Nursing 1996;24:1207-13.

Cheng 2012 \{published data only\} Cheng LSW. The Effects of Mutual Goal Setting on the Outcomes of Care of the Patients in the Community [PhD thesis]. Hong Kong: Hong Kong Polytechnic University, 2012.

Conrad 2000 \{published data only\}

Conrad BC, Glanville NT, Raine-Travers KD. Adherence to a very low fat diet for cardiac rehabilitation patients. Canadian Journal of Dietetic Practice and Research 2000;61: $193-5$.
Coote 2012 \{published data only\}

Coote HM, MacLeod AK. A self-help, positive goalfocused intervention to increase well-being in people with depression. Clinical Psychology and Psychotherapy 2012;19 (4):305-15.

Coppack 2012 \{published data only\}

Coppack RJ, Kristensen J, Karageorghis CI. Use of a goal setting intervention to increase adherence to low back pain rehabilitation: a randomized controlled trial. Clinical Rehabilitation 2012;26(11):1032-42.

Cross 1971 \{published data only\}

Cross JE, Parsons CR. Nurse-teaching and goal-directed nurse-teaching to motivate change in food selection behavior of hospitalized patients. Nursing Research 1971; 20:454-8.

Culley 2010 \{published data only\}

Culley C, Evans JJ. SMS text messaging as a means of increasing recall of therapy goals in brain injury rehabilitation: a single-blind within-subjects trial. Neuropsychological Rehabilitation 2010;20:103-19.

Duncan 2003 \{published data only\}

${ }^{*}$ Duncan K, Pozehl B. Effects of an exercise adherence intervention on outcomes in patients with heart failure. Rehabilitation Nursing 2003;28:117-22.

Duncan KA, Pozehl B. Staying on course: the effects of an adherence facilitation intervention on home exercise participation. Progress in Cardiovascular Nursing 2002;17: 59-65.

Evans 2002 \{published data only\} Evans L, Hardy L. Injury rehabilitation: a goal-setting intervention study. Research Quarterly for Exercise and Sport 2002;73:310-9.

Fredenburgh 1993 \{published data only\} Fredenburgh L. The Effect of Mutual Goal Setting on Stress Reduction in the Community Mental Health Client [Master's thesis]. New York, USA: D’Youville College, 1993.

Gagné 2003 \{published data only\} Gagné DE, Hoppes S. The effects of collaborative goalfocused occupational therapy on self-care skills: A pilot study. American Journal of Occupational Therapy 2003;57: 215-9.

Hart 1978 \{published data only\}

Hart RR. Therapeutic effectiveness of setting and monitoring goals. Journal of Consulting and Clinical Psychology 1978;46:1242-5.

Hart 2002 \{published and unpublished data\} Hart T, Hawkey K, Whyte J. Use of a portable voice organizer to remember therapy goals in traumatic brain injury rehabilitation: a within-subjects trial. Journal of Head Trauma Rehabilitation 2002;17:556-70.

Harwood 2012 \{published data only\} Harwood M, Weatherall M, Talemaitoga A, Barber PA, Gommans J, Taylor W, et al. Taking charge after stroke: 
promoting self-directed rehabilitation to improve quality of life - a randomized controlled trial. Clinical Rehabilitation 2012;26:493-501.

Holliday 2007 \{published data only\} Holliday RC, Cano S, Freeman JA, Playford ED. Should patients participate in clinical decision making? An optimised balance block design controlled study of goal setting in a rehabilitation unit. Journal of Neurology, Neurosurgery and Psychiatry 2007;78:576-80.

Howell 1986 \{published data only\}

Howell C. A controlled trial of goal setting for longterm community psychiatric patients. British Journal of Occupational Therapy 1986;49:264-8.

\section{Iacovino 1997 \{published data only\}}

Iacovino V. A Randomized Comparison Between a GoalSetting and a Videotape and Discussion Intervention to Improve Return to Work and Quality of Life among Cardiac Patients [PhD thesis]. Ottawa, Canada: University of Ottawa, 1997.

\section{James 1993 \{published data only\}}

James LD, Thorn BE, Williams DA. Goal specification in cognitive-behavioral therapy for chronic headache pain. Behavior Therapy 1993; Vol. 24:305-20.

Jonsdottir 2012 \{published data only\} Jonsdottir J, Gervasoni E, Meotti M, Cattaneo D, Montesano A. Efficacy of ICF-based tools in goal setting and the rehabilitation process of people with neurological disorders. Multiple Sclerosis 2012;18(5):S27-8.

* Jonsdottir J, Gervasoni E, Meotti M, Cattaneo D, Montesano A. Efficacy of ICF-based tools in goal setting and the rehabilitation process of people with neurological disorders [Poster]. 17th Annual Conference of Rehabilitation in Multiple Sclerosis; 2012 June; Hamburg 2012.

LaFerriere 1978 \{published data only\}

LaFerriere L, Calsyn R. Goal attainment scaling: an effective treatment technique in short-term therapy. American Journal of Community Psychology 1978;6:271-82.

Mann 1987 \{published data only\}

Mann KV, Sullivan PL. Effect of task-centered instructional programs on hypertensives' ability to achieve and maintain reduced dietary sodium intake. Patient Education and Counseling 1987;10:53-72.

McPherson 2009 \{published and unpublished data\} Kayes N, McPherson KM, Siegert R, McNaughton H, Harwood M. Goal-setting in rehabilitation: a feasibility study of two new approaches in traumatic brain injury. Disability and Rehabilitation 2007;29:1640.

* McPherson KM, Kayes N, Weatherall M. A pilot study of self-regulation informed goal setting in people with traumatic brain injury. Clinical Rehabilitation 2009;23: 296-309.

Miller 2012 \{published data only\}

Miller CK, Headings A, Peyrot M, Nagaraja H. Goal difficulty and goal commitment affect adoption of a lower glycemic index diet in adults with type 2 diabetes. Patient Education and Counseling 2012;86:84-90.

O'Brien 2013 \{published data only\}

O'Brien D, Bassett S, McNair P. The effect of action and coping plans on exercise adherence in people with lower limb osteoarthritis: feasibility study. New Zealand Journal of Physiotherapy 2013;41(2):49-57.

Oestergaard 2012 \{published data only\}

Oestergaard LG, Maribo T, Bünger CE, Christensen FB. The Canadian Occupational Performance Measure's semistructured interview: its applicability to lumbar spinal fusion patients. A prospective randomized clinical study. European Spine Journal 2012;21(1):115-21.

Ostelo 2003 \{published data only\}

Ostelo RWJG, Goossens MEJB, de Vet HCW, van den Brandt PA. Economic evaluation of a behavioral-graded activity program compared to physical therapy for patients following lumbar disc surgery. Spine 2004;29:615-22. Ostelo RWJG, Koke AJA, Beurskens AJHM, de Vet HCW, Kerckhoffs MR, Vlaeyen JW, et al. Behavioralgraded activity compared with usual care after first-time disk surgery: considerations of the design of a randomized clinical trial. Journal of Manipulative and Physiological Therapeutics 2000;23:312-9.

* Ostelo RWJG, de Vet HCW, Vlaeyen JW, Kerckhoffs MR, Berfelo MW, Wolters PMJC, et al. Behavioral graded activity following first-time lumbar disc surgery. Spine 2003;28:1757-65.

Parsons 2012 \{published data only\}

Parsons J, Rouse P, Robinson EM, Sheridan N, Connolly MJ. Goal setting as a feature of homecare services for older people: does it make a difference?. Age and Ageing 2012;41: 24-9.

Richardson 2007 \{published data only\}

Richardson CR, Mehari KS, McIntyre LG, Janney AW, Fortlage LA, Sen A, et al. A randomized trial comparing structured and lifestyle goals in an internetmediated walking program for people with type 2 diabetes. International Journal of Behavioral Nutrition and Physical Activity 2007;4:59.

Scott 2004 \{published data only\} Ranta M. The Effect of Mutual Goal Setting on the SelfEfficacy to Manage Heart Failure in Adults [Master's thesis]. Allendale, MI, USA: Grand Valley State University, 2000. * Scott LD, Setter-Kline K, Britton AS. The effects of nursing interventions to enhance mental health and quality of life among individuals with heart failure. Applied Nursing Research 2004;17:248-56.

Setter-Kline K, Scott LD, Britton AS. The use of supportiveeducative and mutual goal-setting strategies to improve self-management for patients with heart failure. Home Healthcare Nurse 2007;25(8):502-10.

Watson SV. The Effect of Mutual Goal Setting on Perceived Understanding of the Diagnosis of Heart Failure in Adults [Master's thesis]. Allendale MI, USA: Grand Valley State University, 2001. 
Sewell 2005 \{published data only\}

Sewell L, Singh SJ, Williams JEA, Collier R, Morgan

MDL. Can individualized rehabilitation improve functional independence in elderly patients with COPD?. Chest 2005; 128:1194-200.

Stanhope 2013 \{published and unpublished data\}

Stanhope V, Ingoglia C, Schmelter B, Marcus SC. Impact of person-centered planning and collaborative documentation on treatment adherence. Psychiatric Services 2013;64(1): $76-9$.

Taylor 2012 \{published data only\} Taylor WJ, Brown M, Levack W, McPherson KM, Reed K, Dean SG, et al. A pilot cluster randomised controlled trial of structured goal-setting following stroke. Clinical Rehabilitation 2012;26:327-38.

Webb 1994 \{published data only\}

Webb PM, Glueckauf RL. The effects of direct involvement in goal setting on rehabilitation outcome for persons with traumatic brain injuries. Rehabilitation Psychology 1994;39: $179-88$.

Woltmann 2011 \{published data only\}

Woltmann EM, Wilkniss SM, Teachout A, McHugo GJ, Drake RE. Trial of an Electronic Decision Support System to facilitate shared decision making in community mental health. Psychiatric Services 2011; Vol. 62, issue 1:54-60.

\section{References to studies excluded from this review}

\section{Adachi 1989 \{published data only\}}

Adachi Y. The effect of behavioral treatment of obesity and correlates of weight loss in treatment and at 2-year followup. Japanese Journal of Behavior Therapy 1989;15:36-55.

Adair 2013 \{published data only\}

Adair R, Wholey DR, Christianson J, White KM, Britt $\mathrm{H}$, Suhna L. Improving chronic disease care by adding laypersons to the primary care team: a parallel randomized trial. Annals of Internal Medicine 2013;159(3):176-84.

Alfonso 2011 \{published data only\}

Alfonso JP, Caracuel A, Delgado-Pastor LC, Verdejo-Garcia A. Combined goal management training and mindfulness meditation improve executive functions and decisionmaking performance in abstinent polysubstance abusers. Drug and Alcohol Dependence 2011;117:78-81.

Alicea-Planas 2013 \{published data only\}

Alicea-Planas J. Improving self-efficacy for Latinos through goal setting at a community health center. Hispanic Health Care International 2013;11(3):119-30.

Bailey 1988 \{published data only\}

Bailey DB, Simeonsson RJ. Investigation of use of goal attainment scaling to evaluate individual progress of clients with severe and profound mental retardation. Mental Retardation 1988;26:289-95.

Blackberry 2013 \{published data only\} Blackberry ID, Furler JS, Best JD, Chondros P, Vale M, Walker C, et al. Effectiveness of general practice based, practice nurse led telephone coaching on glycaemic control of type 2 diabetes: the Patient Engagement and Coaching for Health $(\mathrm{PEACH})$ pragmatic cluster randomised controlled trial. BMJ 2013;347:f5272.

Bonde 2005 \{published data only\}

Bonde JP, Rasmussen MS, Hjollund H, Svendsen SW, Kolstad HA, Jensen LD, et al. Occupational disorders and return to work: a randomized controlled study. Journal of Rehabilitation Medicine 2005;37:230-5.

Chan 2012 \{published data only\} Chan CK, Cameron LD. Promoting physical activity with goal-oriented mental imagery: a randomized controlled trial. Journal of Behavioral Medicine 2012;35:347-63.

Christiansen 2010 \{published data only\} Christiansen S, Oettingen G, Dahme B, Klinger R. A short goal-pursuit intervention to improve physical capacity: a randomized clinical trial in chronic back pain patients. Pain 2010;149:444-52.

Clare 2013 \{published data only\} Clare L, Bayer A, Burns A, Corbett A, Jones R, Knapp M, et al. Goal-oriented cognitive rehabilitation in early-stage dementia: study protocol for a multi-centre single-blind randomised controlled trial (GREAT). Trials 2013;14:152.

Conrin 1985 \{published data only\}

Conrin JP. A study of methods for implementing a goalsetting system for increasing direct patient contact by psychologists in a state hospital. Dissertation Abstracts International 1985;45:3103.

Curtin 1997 \{published data only\}

Curtin L. Goal setting and feedback in the reduction of heavy drinking in females. Dissertation Abstracts International: Section B: The Sciences and Engineering 1997; 57:4701.

Curtin 2001 \{published data only\} Curtin L, Stephens RS, Bonenberger JL. Goal setting and feedback in the reduction of heavy drinking in female college students. Journal of College Student Psychotherapy 2001;15:17-37.

Drebing 2005 \{published data only\} Drebing CE, Van Ormer EA, Krebs C, Rosenheck R, Rounsaville B, Herz L, et al. The impact of enhanced incentives on vocational rehabilitation outcomes for dually diagnosed veterans. Journal of Applied Behavior Analysis 2005;38:359-72.

Drebing CE, Van Ormer EA, Mueller L, Hebert M, Penk WE, Petry NM, et al. Adding contingency management intervention to vocational rehabilitation: outcomes for dually diagnosed veterans. Journal of Rehabilitation Research and Development 2007;44:851-65.

Duarte 2012 \{published data only\}

Duarte R, Araujo D, Freire L, Folgado H, Fernandes O, Davids K. Intra- and inter-group coordination patterns reveal collective behaviors of football players near the scoring zone. Human Movement Science 2012;31:1639-51.

Estabrooks 2005 \{published data only\} Estabrooks PA, Nelson CC, Xu S, King D, Bayliss EA, Gaglio B, et al. The frequency and behavioral outcomes of 
goal choices in the self-management of diabetes. Diabetes Educator 2005;31:391-400.

Evans-Hudnall 2012 \{published data only\} Evans-Hudnall GL, Stanley MA, Clark AN, Bush AL, Resnicow K, Liu Y, et al. Improving secondary stroke selfcare among underserved ethnic minority individuals: a randomized clinical trial of a pilot intervention. Journal of Behavioral Medicine 2014;37(2):196-204.

Faett 2012 \{published data only\} Faett BL. Remote Delivery of a Standardized Educational Protocol for Self-Management of Chronic Swelling of the Lower Limbs in Individuals with Limited Mobility [PhD thesis]. Ann Arbor: University of Pittsburgh, 2012.

Gorton 2009 \{published data only\}

Gorton JG, Paxton EC, England CY, Coulman KD, Andrews RC. Dietary goal setting in the Early ACTivity In Diabetes (Early ACTID) Study [Conference Presentation]. Diabetic Medicine 2009;March Supplement:165.

Graven 2012 \{published data only\}

Graven C, Brock K, Hill K, Ames D, Cotton S, Joubert L. From rehabilitation to recovery: protocol for a randomised controlled trial evaluating a goal based intervention to reduce depression and facilitate participation post-stroke. BMC Neurology 2011;11:73.

Graven C, Brock K, Hill K, Cotton S, Joubert L. Does a focus on participation and personal goal achievement have an impact on depression in the first year after stroke? Neurorehabilitation and Neural Repair 2012;26(6):755.

Greene 2000 \{published data only\}

Greene JA, Power M, Cane C. Partnership in recovery through goal setting in community rehabilitation following stroke. Consensus Conference on Stroke Treatment and Service Delivery 2000:50.

Hansen 2011 \{published data only\} Hansen LJ, Siersma V, Hornnes HJ, Beck-Nielsen H, De Fine ON. 13-year post-trial follow-up of structured personal care for patients with type 2 diabetes in general practice [Conference Presentation]. Diabetologia 2011;54:S93.

Huang 2010 \{published data only\}

Huang Y, Lee W, Chen S, Yu Y, Chen H. Self-management with goal setting in type 2 diabetes mellitus patients [Chinese]. Journal of Nursing and Healthcare Research 2010; 6:107-16.

Huisman 2010 \{published data only\}

Huisman S, Maes S, De Gucht VJ, Chatrou M, Haak HR. Low goal ownership predicts drop-out from a weight intervention study in overweight patients with type 2 diabetes. International Journal of Behavioral Medicine 2010; 17:176-81.

Huisman S, de Gucht VJ, Maes S, Schroevers M, Chatrou M, Haak HR. Self-regulation and weight reduction in patients with type 2 diabetes: A pilot intervention study. Patient Education and Counselling 2009;75:84-90.

Jeffery 2003 \{published data only\} Jeffery RW, Wing RR, Sherwood NE, Tate DF. Physical activity and weight loss: does prescribing higher physical activity goals improve outcome?. American Journal of Clinical Nutrition 2003;78(4):684-9.

Johnson 2009 \{published data only\}

Johnson ST, Bell GJ, McCargar LJ, Welsh RS, Bell RC. Improved cardiovascular health following a progressive walking and dietary intervention for type 2 diabetes. Diabetes, Obesity and Metabolism 2009;11:836-43.

Katz 2011 \{published data only\}

Katz N, Keren N. Effectiveness of occupational goal intervention for clients with schizophrenia. American Journal of Occupational Therapy 2011;65:287-96.

Kelley 2004 \{published data only\}

Kelley K, Abraham C. RCT of a theory-based intervention promoting healthy eating and physical activity amongst out-patients older than 65 years. Social Science and Medicine 2004;59:787-97.

Kerr 2012 \{published data only\}

Kerr J, Rosenberg DE, Nathan A, Millstein RA, Carlson JA, Crist K, et al. Applying the ecological model of behavior change to a physical activity trial in retirement communities: description of the study protocol. Contemporary Clinical Trials 2012;33:1180-8.

\section{Kerse 2008 \{published data only\}}

Kerse N, Falloon K, Moyes SA, Hayman KJ, Dowell T, Kolt GS, et al. DeLLITE depression in late life: an intervention trial of exercise. Design and recruitment of a randomised controlled trial. BMC Geriatrics 2008;8:12.

Kerse N, Peri K, Robinson E, Wilkinson T, von Randow M, Kiata L, et al. Does a functional activity programme improve function, quality of life, and falls for residents in long term care? Cluster randomised controlled trial. BMJ 2008;337:a1445.

Lenze 2012 \{published data only\}

Lenze EJ, Host HH, Hildebrand MW, Morrow-Howell

N, Carpenter B, Freedland KE, et al. Enhanced

medical rehabilitation increases therapy intensity and engagement and improves functional outcomes in postacute rehabilitation of older adults: a randomized-controlled trial. Journal of the American Medical Directors Association 2012; 13:708-12.

Levine 2007 \{published data only\}

Levine B, Stuss DT, Winocur G, Binns MA, Fahy L, Mandic M, et al. Cognitive rehabilitation in the elderly: effects on strategic behavior in relation to goal management. Journal of the International Neuropsychological Society 2007; 13: $143-52$

Levine 2011 \{published data only\}

Levine B, Schweizer TA, O’Connor C, Turner G, Gillingham S, Stuss DT, et al. Rehabilitation of executive functioning in patients with frontal lobe brain damage with goal management training. Frontiers in Human Neuroscience 2011;5:1-9.

Liang 1984 \{published data only\}

Liang MH, Partridge AJ, Larson MG, Gall V, Taylor

J, Berkman C, et al. Evaluation of comprehensive rehabilitation services for elderly homebound patients with 
arthritis and orthopedic disability. Arthritis and Rheumatism 1984;27:258-66.

Linton 1984 \{published data only\}

Linton SJ, Gotestam KG. A controlled study of the effects of applied relaxation and applied relaxation plus operant procedures in the regulation of chronic pain. British Journal of Clinical Psychology 1984;23:291-9.

Lozano 2010 \{published data only\} Lozano BE, Stephens RS. Comparison of participatively set and assigned goals in the reduction of alcohol use. Psychology of Addictive Behaviors 2010;24:581-91.

Mate-Kole 1999 \{published data only\} Mate-Kole CC, Danquah SA, Twum M, Danquah AO. Outcomes of a nonaversive behavior intervention in intellectually impaired individuals using goal attainment scaling. Nursing Research 1999;48:220-5.

Mitka 2013 \{published data only\}

Mitka M. More patients get good diabetes control, but only a minority meet all goals. JAMA 2013;309:1335-6.

Motl 2012 \{published data only\}

Motl RW, McAuley E, Dlugonski D. Reactivity in baseline accelerometer data from a physical activity behavioral intervention. Health Psychology 2012;31:172-5.

Naik 2011 \{published data only\} Naik AD, Palmer N, Petersen NJ, Street RL Jr, Rao R, Suarez-Almazor M, et al. Comparative effectiveness of goal setting in diabetes mellitus group clinics: randomized clinical trial. Archives of Internal Medicine 2011;171:453-9.

Novakovic-Agopian 2011 \{published data only\} Novakovic-Agopian T, Chen AJW, Rome S, Abrams G, Castelli H, Rossi A, et al. Rehabilitation of executive functioning with training in attention regulation applied to individually defined goals: a pilot study bridging theory, assessment, and treatment. Journal of Head Trauma Rehabilitation 2011;26(5):325-38.

O'Connor 2006 \{published data only\} O'Connor C, Turner GT, Katerji S, Schweizer TA, Black $\mathrm{S}$, Stuss D, et al. A randomized controlled trial of goal management training in adults with neurological damage. 34th International Neuropsychological Society Annual Meeting: From Plasticity to Rehabilitation 2006. 2006: 124.

O'Connor 2008 \{published data only\}

O'Connor J, Seeto C, Saini B, Bosnic-Anticevich S, Krass I, Armour $\mathrm{C}$, et al. Healthcare professional versus patient goal setting in intermittent allergic rhinitis. Patient Education and Counseling 2008;70(1):111-17.

Pandit 2010 \{published data only\}

Pandit A, Bailey S, Davis T, Curtis L, Asada Y, Jain S, et al. The Missouri health literacy and diabetes initiative (MHLDI): helping patients action plan and achieve goals (Conference Presentation). Journal of General Internal Medicine 2010;25:S410.
Pankow 2000 \{published data only\}

Pankow LJ. Evaluating a Multi-Disciplinary Rehabilitation Program for Older Adults with Visual Impairment [PhD thesis]. Athens, GA, USA: University of Georgia, 2000.

Petry 2006 \{published data only\} Petry NM, Alessi SM, Carroll KM, Hanson T, MacKinnon $\mathrm{S}$, Rounsaville B, et al. Contingency management treatments: reinforcing abstinence versus adherence with goal-related activities. Journal of Consulting and Clinical Psychology 2006;74:592-601.

Rodgers 2013 \{published data only\} Rodgers WM, Selzler A-M, Haennel RG, Holm S, Wong EY, Stickland MK. An experimental assessment of the influence of exercise versus social implementation intentions on physical activity during and following pulmonary rehabilitation. Journal of Behavioral Medicine 2013;37(3): 480-90.

Rokke 1999 \{published data only\}

Rokke PD, Tomhave JA, Jocic Z. The role of client choice and target selection in self-management therapy for depression in older adults. Psychology and Aging 1999;14: 155-69.

Smith 2011 \{published data only\}

Smith L, Nguyen T, Seeto C, Saini B, Brown L. The role of non-clinicians in a goal setting model for the management of allergic rhinitis in community pharmacy settings. Patient Education and Counseling 2011;85:e26-32.

Sperduto 1986 \{published data only\} Sperduto WA, Thompson HS, O'Brien RM. The effect of target behavior monitoring on weight loss and completion rate in a behavior modification program for weight reduction. Addictive Behaviors 1986;11:337-40.

Spikman 2010 \{published data only\} Spikman JM, Boelen DHE, Lamberts KF, Brouwer WH, Fasotti L. Effects of a multifaceted treatment program for executive dysfunction after acquired brain injury on indications of executive functioning in daily life. Journal of the International Neuropsychological Society 2010;16:118-29.

Stenstrom 1994 \{published data only\} Stenstrom $\mathrm{CH}$. Home exercise in rheumatoid arthritis functional class II: goal setting versus pain attention. Journal of Rheumatology 1994;21(4):627-34.

St John 1973 \{published data only\} St John D, Soulary E. Introduction of goal-oriented record keeping on an inpatient drug treatment setting. Journal of Psychiatric Nursing and Mental Health Services 1973;11: 20-7.

Stubberud 2013 \{published data only\} Stubberud J, Langenbahn D, Levine B, Stanghelle J, Schanke A-K. Goal management training of executive functions in patients with spina bifida: a randomized controlled trial. Journal of the International Neuropsychological Society 2013; 19:672-85.

Stuifbergen 2003 \{published data only\} Stuifbergen AK, Becker H, Timmerman GM, Kullberg V. The use of individualized goal setting to facilitate behavior 
change in women with multiple sclerosis. Journal of

Neuroscience Nursing 2003;35(2):94-9.

\section{Stuifbergen 2010 \{published data only\}}

Stuifbergen AK, Blozis SA, Becker H, Phillips L,

Timmerman G, Kullberg V, et al. A randomized controlled trial of a wellness intervention for women with fibromyalgia syndrome. Clinical Rehabilitation 2010;24:305-18.

van Hooren 2007 \{published data only\}

van Hooren SAH, Valentijn SAM, Bosma $\mathrm{H}$, Ponds RWHM, van Boxtel MPJ, Levine B, et al. Effect of a structured course involving goal management training in older adults: a randomised controlled trial. Patient Education and Counseling 2007;65:205-13.

Weg 2009 \{published data only\}

Weg J. The Effects of Mutual Goal Setting on Patient Satisfaction among Adult Patients at a Rural Community hospital [Master's thesis]. Brookings SD, USA: South Dakota State University, 2009.

Wilson 2010 \{published data only\}

Wilson SR, Strub P, Buist AS, Knowles SB, Lavori PW, Lapidus J, et al. Shared treatment decision making improves adherence and outcomes in poorly controlled asthma. American Journal of Respiratory and Critical Care Medicine 2010; Vol. 6:566-77.

Wood 2012 \{published data only\}

Wood A, Scott F, Baylan S, Evans J. Rehabilitation of executive function deficits following acquired brain injury: a randomised controlled trial of goal management training and implementation intentions for the improvement of prospective memory [Conference Presentation]. Brain Injury 2012;26(4-5):557.

Wressle 2002 \{published data only\}

Wressle E, Eeg-Olofsson A-M, Marcusson J, Henriksson C. Improved client participation in the rehabilitation process using a client-centred goal formulation structure. Journal of Rehabilitation Medicine 2002;34:5-11.

Zegman 1983 \{published data only\}

Zegman M, Baker B. The influence of proximal vs. distal goals on adherence to prescribed calories. Addictive Behaviors 1983;8:319-22.

\section{References to ongoing studies}

\section{ACTRN12609000433202 \{published data only\}}

ACTRN12609000433202. Goals and self regulation skills in brain injury rehabilitation: A randomised clinical trial. www.anzctr.org.au/Trial/Registration/TrialReview.aspx?id= 83969.

Arends 2013 \{published data only\}

Arends RY, Bode C, Taal E, Van De Laar MA. A goal management intervention for polyarthritis patients: rationale and design of a randomized controlled trial. $B M C$ Musculoskeletal Disorders 2013;14:239.

Bertens 2013 \{published data only\}

Bertens D, Fasotti L, Boelen DHE, Kessels RPC. A randomized controlled trial on errorless learning in goal management training: study rationale and protocol. $B M C$ Neurology 2013;13:64.

Dawson 2013 \{published data only\}

Dawson DR, Anderson ND, Binns MA, Bottari C, Damianakis T, Hunt A, et al. Managing executive dysfunction following acquired brain injury and stroke using an ecologically valid rehabilitation approach: a study protocol for a randomized, controlled trial. Trials 2013;14: 306.

Gracey 2012 \{published data only\}

Gracey F, Wilson BA, Manly T, Bateman A, Fish J, Malley $\mathrm{D}$, et al. The effectiveness of brief goal management training (GMT) and sms text alerts on psychosocial functioning following brain injury: the assisted intention monitoring (AIM) trial?. Brain Impairment 2012;13(1):180-1.

Mansfield 2013 \{published data only\} Mansfield A, Wong JS, Bayley M, Biasin L, Brooks D, Brunton $\mathrm{K}$, et al. Using wireless technology in clinical practice: does feedback of daily walking activity improve walking outcomes of individuals receiving rehabilitation post-stroke? Study protocol for a randomized controlled trial. BMC Neurology 2013;13:93.

Novakovic-Agopian 2012 \{published data only\} Novakovic-Agopian T, Chen A, Abrams G, Rossi A, Binder $\mathrm{D}$, Muir J, et al. Goal-oriented attention regulation training in veterans with chronic TBI [Conference Presentation]. Neurology 2012;78(Meeting Abstracts 1):P01.186.

Reiser 2012 \{published data only\}

Reiser A, Ruddel H, Jurgensen R. So-does it work? A closer look at the effectiveness of an online aftercare program. Psychosomatic Medicine 2012;74(3):A47.

\section{Additional references}

\section{Asenlöf 2009}

Åsenlöf P, Silijebäck K. Goal priority questionnaire is moderately reproducible in people with persistent musculoskeletal pain. Physical Therapy 2009;89:1226-34.

Austin 1996

Austin JT, Vancouver JB. Goal constructs in psychology: structure, process and content. Psychological Bulletin 1996; 120(3):338-75.

Barnes 2000

Barnes MP, Ward AB. Textbook of Rehabilitation Medicine. Oxford: Oxford University Press, 2000.

\section{Barnett 1999}

Barnett D. The rehabilitation nurse as educator. In: Smith M editor(s). Rehabilitation in Adult Nursing Practice. Edinburgh: Churchill Livingstone, 1999:53-76.

\section{Bartholomew 1998}

Bartholomew LK, Parcel GS, Kok G. Intervention mapping A process for developing theory and evidence-based health education programs. Health Education and Behavior 1998; 25(5):545-63. 
Black 2010

Black JS, Brock KA, Kennedy G, Mackenzie M. Is achievement of short-term goals a valid measure of patient progress in inpatient neurological rehabilitation?. Clinical Rehabilitation 2010;24:373-9.

Boekaerts 2000

Boekaerts M, Niemivirta M. Self-regulated learning: finding a balance between learning goals and ego-protective goals. In: Boekaerts M, Pintrich PR, Zeidner M editor(s). Handbook of Self-regulation. San Diego: Academic Press, 2000:417-51.

\section{Bovend'Eerdt 2009}

Bovend'Eerdt TJH, Botell RE, Wade DT. Writing SMART rehabilitation goals and achieving goal attainment scaling: a practical guide. Clinical Rehabilitation 2009;23:352-61.

\section{Brennan 2009}

Brennan S, McKenzie J, Whitty P, Buchan H, Green S. Continuous quality improvement: effects on professional practice and healthcare outcomes. Cochrane Database of Systematic Reviews 2009, Issue 4. [DOI: 10.1002/ 14651858.CD003319.pub2]

Burton 2010

Burton D, Pickering M, Weinberg R, Yukelson D, Weigand, $D$. The competitive goal effectiveness paradox revisited: examining the goal practices of prospective Olympic athletes. Journal of Applied Sports Psychology 2010;22:72-86.

\section{Clarke 2006}

Clarke SP, Oades LG, Crowe TP, Deane FP. Collaborative Goal Technology: theory and practice. Psychiatric Rehabilitation 2006;30:129-36.

\section{Craig 2008}

Craig P, Dieppe P, Macintyre S, Michie S, Nazareth I, Petticrew M. Developing and evaluating complex interventions: The new Medical Research Council guidance. BMJ 2008;337:979-83.

\section{Custers 2010}

Custers R, Aarts H. The unconscious will: how the pursuit of goals operates outside of conscious awareness. Science 2010;329:47-50.

Cytrynbaum 1979

Cytrynbaum S, Ginath Y, Birdwell J, Brandt L. Goal attainment scaling: a critical review. Evaluation Quarterly 1979;3:5-40.

\section{Duncan 2005}

Duncan PW, Zorowitz RD, Bates B, Choi JY, Glasberg JJ, Graham GD, et al. AHA/ASA-endorsed practice guidelines: Management of Adult Stroke Rehabilitation Care: A Clinical Practice Guideline. Stroke 2005;36:e100-43.

\section{Evans 2001}

Evans PJ, Zinkin P, Harpham T, Chaudury G. Evaluation of medical rehabilitation in community based rehabilitation. Social Science and Medicine 2001;53:333-48.

\section{Gauggel 2001}

Gauggel S, Leinberger R, Richardt M. Goal setting and reaction time performance in brain-damaged patients.
Journal of Clinical and Experimental Neuropsychology 2001; 23(3):351-61.

Glasgow 2000

Glasgow RE, Toobert DJ. Brief, computer-assisted diabetes dietary self-management counseling: effects on behavior, physiologic outcomes, and quality of life. Medical Care 2000;38(11):1062-73.

Hall 2001

Hall HK, Kerr AW. Goal setting in sport and physical activity: tracing empirical developments and establishing conceptual direction. In: Roberts GC editor(s). Advances in Motivation in Sport and Exercise. Champaign, US: Human Kinetic Publishers, Inc, 2001.

\section{Higgins 2003}

Higgins JPT, Thompson SG, Deeks JJ, Altman DG. Measuring inconsistency in meta-analysis. BMJ 2003;327: $557-60$.

\section{Higgins 2011}

Higgins JPT, Green S (editors). Cochrane Handbook for Systematic Reviews of Interventions Version 5.1.0 [updated March 2011]. The Cochrane Collaboration, 2011. Available from www.cochrane-handbook.org.

\section{Horvat 2014}

Horvat, L, Horey, D, Romios P, Kis-Rigo J. Cultural competence education for health professionals. Cochrane Database of Systematic Reviews 2014, Issue 5. [DOI: 10.1002/14651858.CD009405.pub2]

\section{King 1981}

King IM. A Theory for Nursing: Systems, Concepts, Process. New York: Wiley, 1981.

\section{Kiresuk 1968}

Kiresuk T, Sherman R. Goal attainment scaling: a general method for evaluating community health programs. Community Mental Health Journal 1968;4:443-53.

\section{Kok 2004}

Kok G, Schaalma H, Ruiter RA, Van Empelen P, Brug J. Intervention mapping: Protocol for applying health psychology theory to prevention programmes. Journal of Health Psychology 2004;9(1):85-98.

Latham 2007

Latham GP, Locke EA. New developments in and directions for goal-setting research. European Psychologist 2007;12(4): 290-300.

\section{Levack 2006a}

Levack WMM, Taylor K, Siegert RJ, Dean SG, McPherson $\mathrm{KM}$, Weatherall M. Is goal planning in rehabilitation effective? A systematic review. Clinical Rehabilitation 2006; 20:739-55.

\section{Levack 2006b}

Levack WMM, Dean SG, McPherson KM, Siegert RJ. How clinicians talk about the application of goal planning to rehabilitation for people with brain injury - variable interpretations of value and purpose. Brain Injury 2006;20: 1439-49. 


\section{Levack 2006c}

Levack WMM, Dean SG, Siegert RJ, McPherson KM. Purposes and mechanisms of goal planning in rehabilitation: the need for a critical distinction. Disability and Rehabilitation 2006;28:741-9.

\section{Levack 2009}

Levack WMM, Siegert RJ, Dean SG, McPherson KM. Goal planning for adults with acquired brain injury: how clinicians talk about involving family. Brain Injury 2009; 23:192-202.

\section{Levack 2014a}

Levack WMM, Siegert RS. Challenges in theory, practice, and evidence. In: Siegert RJ, Levack WMM editor(s). Rehabilitation Goal Setting: Theory, Practice, and Evidence. London: Taylor \& Francis Group, 2014:3-20.

\section{Levack 2014b}

Levack WMM, Siegert RJ, Pickering N. Ethics and goal setting. In: Siegert RJ, Levack WMM editor(s). Rehabilitation Goal Setting: Theory, Practice and Evidence. London: Taylor \& Francis Group, 2014:67-87.

\section{Levack 2014c}

Levack WMM, Dean SG, McPherson KM, Siegert RJ. Evidence-based goal setting: cultivating the science of rehabilitation. In: Siegert RJ, Levack WMM editor(s). Rehabilitation Goal Setting: Theory, Practice and Evidence. Taylor \& Francis Group, 2014:21-44.

\section{Levine 2000}

Levine B, Robertson IH, Clare L, Carter G, Hong J, Wilson BA, et al. Rehabilitation of executive functioning: an experimental-clinical validation of goal management training. Journal of the International Neuropsychological Society 2000;6:299-312.

\section{Locke 2002}

Locke EA, Latham GP. Building a practically useful theory of goal setting and task motivation: a 35-year odyssey. American Psychologist 2002;57(9):705-17.

Malec 1999

Malec JF. Goal attainment scaling in rehabilitation. Neuropsychological Rehabilitation 1999;9:253-75.

\section{Marsland 2010}

Marsland E, Bowman J. An interactive education session and follow-up support as a strategy to improve clinicians' goal-writing skills: a randomized controlled trial. Journal of Evaluation in Clinical Practice 2010;16:3-13.

\section{Mastos 2007}

Mastos M, Miller K, Eliasson AC, Imms C. Goal-directed training: linking theories of treatment to clinical practice for improved functional activities in daily life. Clinical Rehabilitation 2007;21:47-55.

\section{McLellan 1997}

McLellan DL. Introduction to rehabilitation. In: Wilson BA, McLellan DL editor(s). Rehabilitation Studies Handbook. Cambridge: Cambridge University Press, 1997: $1-19$.

\section{McMillan 1999}

McMillan TM, Sparkes C. Goal planning and neurorehabilitation: the Wolfson Neurorehabilitation Centre approach. Neuropsychological Rehabilitation 1999;9: 241-51.

\section{McPherson 2014}

McPherson KM, Kayes NM, Kersten P. MEANING as a smarter approach to goals in rehabilitation. In: Siegert RJ, Levack WMM editor(s). Rehabilitation Goal Setting: Theory, Practice, and Evidence. London: Taylor \& Francis Group, 2014:105-119.

Melville 2002

Melville LL, Baltic TA, Bettcher TW, Nelson DL. Patients' perspectives on the self-identified goals assessment. American Journal of Occupational Therapy 2002;56:650-9.

\section{Monaghan 2005}

Monaghan J, Channell K, McDowell D, Sharma AK. Improving patient and carer communication, multidisciplinary team working and goal-setting in stroke rehabilitation. Clinical Rehabilitation 2005;19:194-9.

Moore 2015

Moore GF, Audrey S, Barker M, Bond L, Bonell C, Hardeman W, et al. Process evaluation of complex interventions: Medical Research Council guidance. BMJ 2015;350:h1258.

Moskowitz 2009

Moskowitz GB, Grant H. The Psychology of Goals. New York, NY: The Guilford Press, 2009.

Pendleton 2005

Pendleton HM, Schultz-Krohn W. Pedrettis Occupational Therapy Practice Skills for Physical Dysfunction. 6th Edition. St Louis, Missouri: Mosby Elsevier, 2005.

\section{Phipps 2007}

Phipps S, Richardson P. Occupational therapy outcomes for clients with traumatic brain injury and stroke using the Canadian Occupational Performance Measure. American Journal of Occupational Therapy 2007;61(3):328-34.

\section{Pintrich 2000}

Pintrich, PR. The role of goal orientation in self-regulated learning. In: Boekaerts M, Pintrich PR, Zeidner M editor (s). Handbook of Self-Regulation. San Diego: Academic Press, 2000:451-502.

\section{Playford 2009}

Playford ED, Siegert RJ, Levack W, Freeman J. Areas of consensus and disagreement about goal-setting in rehabilitation: a conference report. Clinical Rehabilitation 2009;23:334-44.

\section{Poltawski 2014}

Poltawski L, Norris M, Dean S. Intervention fidelity: developing an experience-based model for rehabilitation research. Journal of Rehabilitation Medicine 2014;46: 609-15.

\section{Powell 2002}

Powell J, Heslin J, Greenwood R. Community based rehabilitation after severe traumatic brain injury: a 
randomised controlled trial. Journal of Neurology,

Neurosurgery and Psychiatry 2002;72(2):193-202.

\section{Randall 2000}

Randall KE, McEwen IR. Writing patient-centered functional goals. Physical Therapy 2000;80:1197-203.

\section{RCP 2003}

Royal College of Physicians and British Society of Rehabilitation Medicine. Rehabilitation Following Acquired Brain Injury: National Clinical Guidelines. London: RCP, BSRM, 2003.

\section{RCP 2004}

Royal College of Physicians of London. National Clinical Guidelines for Stroke. 2nd Edition. London: Royal College of Physicians of London, 2004.

\section{RevMan 2014}

The Nordic Cochrane Centre, The Cochrane Collaboration. Review Manager (RevMan). 5.3. Copenhagen: The Nordic Cochrane Centre, The Cochrane Collaboration, 2014.

\section{Rosewilliam 2011}

Rosewilliam S, Roskell CA, Pandyan AD. A systematic review and synthesis of the quantitative and qualitative evidence behind patient-centred goal setting in stroke rehabilitation. Clinical Rehabilitation 2011;25(6):501-14.

\section{Rothstein 2003}

Rothstein JM, Echternach JL, Riddle DL. The hypothesisoriented algorithm for clinicians II (HOAC II): a guide for patient management. Physical Therapy 2003;83:445-70.

\section{Ryan 2011}

Ryan R, Hill S, Prictor M, McKenzie J. Study Quality Guide. www.latrobe.edu.au/chcp/cochrane/resources.html (accessed 16 Dec 2011).

\section{Schut 1994}

Schut HA, Stam HJ. Goals in rehabilitation teamwork. Disability and Rehabilitation 1994;16:223-6.

\section{Scobbie 2009}

Scobbie L, Wyke S, Dixon D. Identifying and applying psychological theory to setting and achieving rehabilitation goals. Clinical Rehabilitation 2009;23:321-33.

\section{Siegert 2004}

Siegert RJ, McPherson KM, Taylor W. Toward a cognitiveaffective model of goal-setting in rehabilitation: is selfregulation theory a key step?. Disability and Rehabilitation 2004;26:1175-83.

\section{Siegert 2014a}

Siegert RJ, O'Connell C, Levack WMM. Psychology, goals and rehabilitation - providing a theoretical foundation. In: Siegert RJ, Levack WMM editor(s). Rehabilitation Goal Setting: Theory, Practice, and Evidence. London: Taylor \& Francis Group, 2014:45-66.

\section{Siegert 2014b}

Siegert RS, Levack WMM, editors. Rehabilitation Goal Setting: Theory, Practice, and Evidence. London: Taylor \& Francis Group, 2014.

\section{Steenbeek 2007}

Steenbeek D, Ketelaar M, Galama K, Gorter JW. Goal attainment scaling in paediatric rehabilitation: a critical review. Developmental Medicine and Child Neurology 2007; 49:550-6.

Sugavanam 2013

Sugavanam T, Mead G, Bulley C, Donaghy M, Van Wijck F. The effects and experiences of goal setting in stroke rehabilitation - a systematic review. Disability and Rehabilitation 2013;35(5):177-90.

\section{Sullivan 2006}

Sullivan MJ, Adams H, Rhodenizer T, Stanish WD. A psychosocial risk factor-targeted intervention for the prevention of chronic pain and disability following whiplash injury. Physical Therapy 2006;86:8-18.

\section{Tennant 2007}

Tennant A. Goal attainment scaling: current methodological challenges. Disability and Rehabilitation 2007;29(20-21): 1583-8.

\section{Trombly 1999}

Trombly CA, Wu CY. Effect of rehabilitation tasks on organization of movement after stroke. American Journal of Occupational Therapy 1999;53(4):333-44.

Trombly 2002

Trombly CA, Radomski MV, Trexel C, Burnet-Smith SE. Occupational therapy and achievement of self-identified goals by adults with acquired brain injury: phase II. American Journal of Occupational Therapy 2002;56(5): 489-98.

\section{Turner-Stokes 2009}

Turner-Stokes L. Goal attainment scaling (GAS) in rehabilitation: a practical guide. Clinical Rehabilitation 2009;23:362-70.

\section{Turner-Stokes 2010}

Turner-Stokes L, Williams H. Goal attainment scaling: a direct comparison of alternative rating methods. Clinical Rehabilitation 2010;24:66-73.

\section{Visser-Meily 2006}

Visser-Meily A, Post M, Gorter JW, Berlekom SBV, Bos VD, Lindeman E. Rehabilitation of stroke patients needs a family-centred approach. Disability and Rehabilitation 2006;28:1557-61.

\section{Wade 1998}

Wade DT. Evidence relating to goal planning in rehabilitation. Clinical Rehabilitation 1998;12(4):273-5.

\section{Wade 1999a}

Wade DT. Goal planning in stroke rehabilitation: how?. Topics in Stroke Rehabilitation 1999;6:16-36.

\section{Wade 2009}

Wade DT. Goal setting in rehabilitation: an overview of what, why and how. Clinical Rehabilitation 2009;23:291-5.

\section{WHO 1992}

World Health Organization. International Statistical Classification of Diseases and Related Health Problems. Geneva: World Health Organization, 1992. 


\section{WHO 2001a}

World Health Organization. International Classification of Functioning, Disability and Health. Geneva: World Health Organization, 2001.

\section{WHO 2001b}

World Health Organization. The UN Standard Rules on the Equalization of Opportunities for Persons with Disabilities. II. Main Report. Geneva: World Health Organization, 2001.

\section{Willer 1976}

Willer B, Miller GH. Client involvement in goal setting and its relationship to therapeutic outcome. Journal of Clinical Psychology 1976;32:687-90.

\section{Wilson 2006}

Wilson KM, Hardy L, Harwood CG. Investigating the relationship between achievement goals and process goals in rugby union players. Journal of Applied Sports Psychology 2006; 18:297-311.

\section{Wilson 2008}

Wilson BA. Neuropsychological rehabilitation. Annual Review of Clinical Psychology 2008;4:141-62.

\section{Wressle 2003}

Wressle E, Lindstrand J, Neher M, Marcusson J, Henriksson C. The Canadian Occupational Performance Measure as an outcome measure and team tool in a day treatment programme. Disability and Rehabilitation 2003;25(10): 497-506.

\section{References to other published versions of this review}

\section{Levack 2012}

Levack WMM, Siegert RJ, Dean SG, McPherson K, HaySmith EJC, Weatherall MM. Goal setting and activities to enhance goal pursuit for adults with acquired disabilities participating in rehabilitation. Cochrane Database of Systematic Reviews 2012, Issue 4. [DOI: 10.1002/ 14651858.CD009727]

* Indicates the major publication for the study 


\title{
CHARACTERISTICS OF STUDIES
}

\section{Characteristics of included studies [ordered by study ID]}

\author{
Arnetz 2004
}

Methods

Quasi-RCT

Setting: A department of rheumatology at a university hospital, Sweden

Funding: Supported by the Örebro University Hospital, Sweden

Recruitment (patients): 'All patients admitted to the rheumatology rehabilitation unit between September of 1996 and September 1997, a total of 82 individuals, were asked to participate in the study' (p.52)

Recruitment (healthcare providers): All physical therapists (total two) employed as staff members in the rheumatology rehabilitation unit were involved in the study

Inclusion criteria (patients): All patients admitted to the rheumatology rehabilitation unit

Exclusion criteria (patients): Not further criteria reported

Consideration of people with comorbidities: Not reported

Participants

Patients: 77 participants (27.3\% male); 27\% under or equal to 45 years, $73 \%$ over 45 . Mean age and SD not reported. Ethnicity not reported

Principle health problems: Musculoskeletal disorders (27\% ankylosing spondylitis; 67\% rheumatoid arthritis; $5 \%$ psoriatic arthritis; $1 \%$ other condition). $51 \%$ treated as inpatients; $49 \%$ outpatient. $74 \%$ previously treated at the unit; $26 \%$ new referrals

Treatment currently receiving: Not reported

Description of healthcare providers: Two physiotherapists, one assigned to each group. Demographic data on the treating therapists were not reported involved in establishing their goals for physical therapy would: 1) achieve better physical treatment outcomes, such as range of motion, strength, balance, overall fitness, walking and functional ability; and 2) give higher ratings to the quality of physical therapy care Intervention: ( $\mathrm{n}=39$ ) Physical therapy care plus goal planning. Patients completed a patient goal checklist on their first meeting with the physical therapist, in which they selected from a list of pre-established goal options. The therapist independently completed the same checklist for each patient. The two checklists were compared at a 'goal forum', with collaborative discussion resulting in agreement on treatment goals in three areas: pain, physical ability, and functional ability

Control: $(\mathrm{n}=38)$ Patients received physical therapy according to the traditional model. 'In our definition of traditional therapy, the patient describes and explains both situation and symptoms. The extent to which the patient is then involved in treatment decisions is very much dependent upon the individual physical therapist and/or the individual patient.' (p.51)

Delivery: Data on delivery of physical therapy (e.g. number of sessions, duration of sessions, frequency of session, content of sessions) was not reported, nor what other treatment was being provided in the rheumatology rehabilitation unit

Fidelity: Not reported

Consumer involvement outside of the intervention: Physical therapists and patients, not otherwise involved in the study, were consulted with on design of the goal setting method

Goal setting and strategies to enhance goal pursuit for adults with acquired disability participating in rehabilitation (Review) 


\begin{tabular}{|c|c|}
\hline Outcomes & $\begin{array}{l}\text { Timing of outcomes: On completion of treatment (timeframe or number of treatments } \\
\text { not specified) } \\
\text { General treatment outcome (10-point Likert scale) } \\
\text { Goal achievement (agreement between 'target' and 'actual' outcomes on a 5-point scale) } \\
\text { Quality of care, self-reported (24-item questionnaire) }\end{array}$ \\
\hline Goal setting characteristics & $\begin{array}{l}\text { Comparison of interest: Collaborative goal setting versus usual care with no standardised } \\
\text { or required approach to goal setting } \\
\text { Patient involvement in goal setting: Goals collaboratively set by the patient and healthcare } \\
\text { professional } \\
\text { Family involvement in goal setting: None reported } \\
\text { Name of goal setting approach: No specifically named approach used } \\
\text { Development of a plan for goal pursuit: Not reported } \\
\text { Written copy of goals provided to patients: Not reported } \\
\text { Individual versus group-based goal discussion: Individual } \\
\text { Level of goal difficulty: Not reported } \\
\text { Goal areas of focus: Selected from a checklist related to impairments of body structure, } \\
\text { body function, and activity limitations } \\
\text { Evaluation of patient goal commitment: Not reported } \\
\text { Goal reminders used: Not reported } \\
\text { Monitoring of progress towards goals: Not reported }\end{array}$ \\
\hline
\end{tabular}

Notes

Power calculation: 'Based on a minimum of 30 respondents per group, the power to detect a $10 \%$ difference in the overall quality scales between groups was estimated at 0 . $95^{\prime}$ (p. 54)

\section{Risk of bias}

\begin{tabular}{|c|c|c|}
\hline Bias & Authors' judgement & Support for judgement \\
\hline $\begin{array}{l}\text { Random sequence generation (selection } \\
\text { bias) }\end{array}$ & High risk & $\begin{array}{l}\text { 'Each of the two physical therapists was } \\
\text { solely responsible for each respective group. } \\
\text { ' (p. 52) '... since only two physical thera- } \\
\text { pists were involved in the study, alternate } \\
\text { patients were assigned to PT1 and then } \\
\text { PT2 upon admission' (author communica- } \\
\text { tion) }\end{array}$ \\
\hline Allocation concealment (selection bias) & High risk & See above \\
\hline $\begin{array}{l}\text { Blinding of participants and personnel } \\
\text { (performance bias) } \\
\text { All outcomes }\end{array}$ & High risk & $\begin{array}{l}\text { '... there was a possibility of bias on the } \\
\text { part of both patients and therapists, since } \\
\text { neither was blinded to the purpose of the } \\
\text { study' (p.59). The intervention required ac- } \\
\text { tive involvement of the patient and health- } \\
\text { care professionals, so blinding not possible }\end{array}$ \\
\hline
\end{tabular}




\begin{tabular}{|c|c|c|}
\hline $\begin{array}{l}\text { Blinding of outcome assessment (detection } \\
\text { bias) } \\
\text { All outcomes }\end{array}$ & High risk & $\begin{array}{l}\text { All outcomes evaluated by patients or ther- } \\
\text { apists involved in delivery of the interven- } \\
\text { tions }\end{array}$ \\
\hline
\end{tabular}

Incomplete outcome data (attrition bias) Low risk All outcomes

Selective reporting (reporting bias)

Unclear risk
Questionnaires received from 75 of the 77 participants. '... all patient goals that had been set were maintained and measured at the conclusion of the treatment period' (author communication)

Not all outcome data are available for analysis. Data on goal achievement is incomplete as percentages are reported without information on the absolute values making up those percentages. Outcomes included a 24-item questionnaire, from which values for only three items are reported as they were found to produce a statistically significant difference between groups

Other bias

High risk

RCT

Setting: Three physical therapy clinics in the primary healthcare sector, Sweden

Funding: Unclear, but it is stated that the study was supported by Swedish Research Council and Swedish Council for Research on Technology Assessment in Healthcare

Recruitment (patients): Patients recruited by three administrative assistants from the participating physical therapy clinics. Assessed for eligibility: 229; of these 10 excluded, 97 declined to participate

Recruitment (healthcare providers): Not stated

Inclusion criteria (patients): Aged between 18 to 65 years, with persistent musculoskeletal pain for more than four weeks, consulting with physiotherapists in primary healthcare settings between Feb 2003 and Feb 2004, literate in Swedish

Exclusion criteria (patients): recent traumas (e.g. whip lash-associated disorders), rheumatic, neurologic, or malignant diseases, ongoing medical or psychological treatment for depression, or having received treatment by a physical therapist during the previous six months

Consideration of people with comorbidities: Excluded by recruitment criteria

Participants

Patients: 122 participants enrolled. 97 participants received the study intervention (22. $7 \%$ male). Mean age 41.6 (intervention group); 43.4 years (control group) (SD 12.4 experimental group; 10.9 control group). $92.6 \%$ Swedish; $7.4 \%$ other ethnicity 
Principle health problems: Persistent pain conditions - 25.8\% low back pain; 3.1\% neck pain; $10.3 \%$ shoulder, arm or hand pain; $2.1 \%$ hip, knee, or foot pain; $5.2 \%$ other single pain site; $53.5 \%$ more than two pain sites

Treatment currently receiving: No treatment in addition to physical therapy intervention as part of the study

Description of healthcare providers: Eight physical therapists, allocation to either the intervention or control group treatment protocol, 2 to 32 years clinical experience

Interventions

Study aim: To test the hypothesis that an individually tailored behavioural medicine intervention (based on goal setting and strategies to enhance goal pursuit) would be more effective than exercise therapy in terms of reducing pain-related disability in a population of people receiving a physical therapy for musculoskeletal pain in a primary healthcare setting

Intervention: ( $\mathrm{n}=57)$ Individually-tailored, goal-oriented behavioural medicine sessions, plus two booster sessions after one and three months. Therapy involved: 1) establishment of a prioritised list of patient goals using the Patient Goal Priority Questionnaire; 2) selfmonitoring of target activities and psychosocial factors related activity performance; 3 ) individual functional behaviour analysis - to identify the 'important, controllable, causal function relationships' (Åsenlöf et al, 2005, p. 592) underpinning the target behaviours, resulting in further specification of the treatment goals and basic skills exercises; 4) exercise, activities, and cognitive skills training to meet specified goals; 5) application of skills acquisition, in the physical therapy clinic then in home environment; 6) generalisation (following attainment of one goal, the next of the prioritised list was targeted) ; 7) maintenance and relapse prevention - identification and management of high-risk situations

Control: $(n=65)$ Individualised, standard physical therapy exercise for chronic pain, excluding any behavioural goal-related intervention (i.e. any functional behavioural analyses, cognitive skills training, applied activities training, maintenance and relapse prevention strategies)

Delivery: Eight to ten supervised physical therapy sessions over a 2 to 3 month period Fidelity: Several strategies used to enhance and monitor treatment fidelity including: patient reports of treatment content, therapists' documentation of treatment content for each session, auditing of individual working sheets and exercise sheets, monitoring of number and duration of treatment sessions

Consumer involvement outside of the intervention: Not stated

Outcomes

Timing of data collection: Baseline, end of treatment (after 8 to 10 sessions), and three months after treatment completion

Pain Disability Index

Average, maximal, and mildest pain intensity during the past two weeks

Perceived pain control

Swedish version of the Self-Efficacy Scale

Swedish version of the Tampa Scale of Kinesiophobia

Five physical performance tests: (sit-ups, push-ups, back-ups, functional lifting ability, number of step climbed in 35 seconds)

Global rating of improvement, self-reported

Patient satisfaction with daily living

Patient satisfaction with treatment

Patient confidence in self-management of future risk situations

Goal setting and strategies to enhance goal pursuit for adults with acquired disability participating in rehabilitation (Review) 
Asenlof 2005 (Continued)

Patient self-reporting of application of learned skills

Goal setting characteristics

Comparison of interest: Collaborative goal setting plus strategies to enhance goal pursuit versus usual care with no standardised or required approach to goal setting

Patient involvement in goal setting: Goals collaboratively set by the patient and healthcare professional

Family involvement in goal setting: None reported

Name of goal setting approach: Based on use of the Patient Goal Priority Questionnaire

Development of a plan for goal pursuit: Yes

Written copy of goals provided to patients: Yes

Individual versus group-based goal discussion: Individual

Level of goal difficulty: Not specified by approach

Goal areas of focus: Not specified by approach

Evaluation of patient goal commitment: Not reported

Goal reminders used: Regular discussion of goals and solution for barriers to goal achievement plus use of goal 'homework assignment' as a regular part of therapy

Monitoring of progress towards goals: Discussion of goal progress as a regular part of therapy

Notes

Power calculation: Conducted a priori, based on an estimate 'that a sample size of 140 participants would be desirable to avoid Type II errors. The number of participants was based on estimates of 0.8 power to detect a medium effect size at $\mathrm{p}=0.5^{\prime}$ ( $\AA$ senlöf et al, 2005, p.596). Which outcome this power calculation was directed towards was unclear however, although the primary outcome for the study is stated as being the Pain Disability Index (PDI). 122 participants were recruited and randomised at the beginning of the study, with 97 receiving the intervention as prescribed

\section{Risk of bias}

\section{Bias}

bias)

Allocation concealment (selection bias)
Authors' judgement

Low risk

Low risk

\section{Support for judgement}

Randomisation conducted by way of a 'random digit table’ (Åsenlöf et al, 2005, p.592)

'The randomization sequences were concealed to the research personnel who recruited, measured, and treated patients. Two researchers, not involved in these procedures, generated the randomization sequences and stored them in a place not accessible for other research personnel. When a patient was recruited at any of the three centres and baseline measures were completed and registered, the recruiting personnel phoned the researcher with access to the randomizations sequences and got the allocation for this particular patient according to the randomization list' (author communication) 
Blinding of participants and personnel High risk (performance bias)

Blinding of outcome assessment (detection High risk bias)

All outcomes

Incomplete outcome data (attrition bias)

High risk

All outcomes
All outcomes

The intervention required active involvement of the patient and healthcare professionals, so blinding not possible

Most outcomes evaluated on the basis of patient self-report (not blinded to the intervention received). The therapists who performed the physical performance outcome assessments were also not blinded to group allocation (author communication)

Intention-to-treat analysis followed, but dropout rates were nonetheless very high: $20.5 \%(25 / 122)$ attrition by completion of treatment; $33.6 \%(41 / 122)$ attrition by the three-month follow up; $46.7 \%$ (57/ 122) by the two-year follow up. Of the participants remaining at three months: 'Occasional missing items in the separate questionnaires were substituted with the mean of each individual's total item score. Questionnaires unanswered as a whole were handled with intention-to-treat' analysis. All analyses including self-report measures were conducted in 2 ways: (1) intentionto-treat analyses including all randomized participants with eligible baseline measures (Fig 1) (the longitudinal imputation method of last value carried forward was used for this purpose), and (2) analyses including those who completed treatment only. Because both ways of analyses revealed the same overall effects, the latter are reported in the results section' (Assenlöf et al, 2005, p.596). A similar approach was taken for the two-year follow-up data analysis, plus a third approach involving having 'missing values replaced with the worst 10th percentile of scores within each condition’ (Åsenlöf et al, 2009, p.1085)

Selective reporting (reporting bias) Unclear risk
Unable to find a protocol for this study published prior to it being conducted, so unable to compare the outcomes reported with those planned to be measured at the outset 
Asenlof 2005 (Continued)

Other bias

Low risk
Treatment fidelity methods used in the study limited risk of bias arising from crossgroup contamination. No other sources of bias were apparent

Bassett 1999

Methods

Participants
RCT

Setting: Private and publicly-funded physiotherapy clinics, New Zealand

Funding: Maurice and Phyllis Paykel Trust $\&$ the New Zealand Society of Physiotherapists Scholarship Trust

Recruitment (patients): Recruited by the participating physiotherapists

Recruitment (healthcare providers): Not reported

Inclusion criteria (patients): Starting new course of physiotherapy for limb injury, which required exercise to practise at home

Exclusion criteria (patients): No further criteria reported

Consideration of people with comorbidities: Not reported

Patients: 74 participants enrolled. 66 participants completed their course of physiotherapy ( $48.5 \%$ male). Mean age 41 (SD 16). Range 13 to 72 . Ethnicity not stated

Principle health problems: Musculoskeletal disorders (40.9\% upper limb injuries; 59. $1 \%$ lower limb injuries)

Treatment currently receiving: Physiotherapy

Description of healthcare providers: 17 physiotherapists (15 in private sector; two in public sector)

Interventions

Study aim: To test the hypothesis that physiotherapy patients who participate in collaborative goal setting will have a higher level of compliance with their home exercise than those who have physiotherapist-mandated goal and those who have no formally set goals Intervention (Collaborative goal setting): $(n=25)$ Participant-physiotherapist collaborative goal setting

Long-term goals established during the initial treatment session, which were broken down into more immediate, achievable short-term goals. Goals altered at subsequent meeting to meet the changing needs of the participants' conditions. Both the participants and the physiotherapists were involved in setting the goals for those in this condition. Goals were based on daily functional activities the participants wished to achieve, and what the physiotherapist thought was realistic

Intervention (mandated goal setting): ( $\mathrm{n}=24)$ Physiotherapist-mandated goal setting. The same treatment was received as for the intervention group, but with only the physiotherapist selecting the goals for treatment, with rewording of these goals into a language that the patient understood

Control: $(\mathrm{n}=25)$ Physiotherapy treatment with no goals set

Delivery: No restrictions reported on the duration or number of treatment sessions Fidelity: Providers given oral and written instruction in the purpose of the study, plus a written booklet on the study methods, the two types of goal setting, use of exercise diaries, and use of the measuring instruments. No evaluation of intervention fidelity was reported however

Consumer involvement outside of the intervention: None reported 
Outcomes

Goal setting characteristics
Timing of outcomes: On enrolment in study and on completion of treatment. Mean number of treatment sessions: 12.79 (SD 8.33). Duration of treatment not reported Completion of home exercises, self-reported

Rate of symptom relief (measured by number of treatments required)

Degree of symptom relief, self-reported

Percentage improvement in range of movement

Percentage improvement in muscle strength

$\begin{array}{ll} & \text { versus no goal setting } \\ \text { Patient involvement in goal setting: The study compared goal set in collaboration with } \\ \text { patients versus those set by a healthcare professional for patients (versus no goal setting) } \\ \text { Family involvement in goal setting: None reported } \\ \text { Name of goal setting approach: No specifically named approach used } \\ \text { Development of a plan for goal pursuit: Not reported } \\ \text { Written copy of goals provided to patients: Patients were given exercise diaries and goal } \\ \text { sheets for the self-reporting of progress } \\ \text { Individual versus group-based goal discussion: Individual } \\ \begin{array}{l}\text { Level of goal difficulty: Achievable, realistic goals were emphasised } \\ \text { Goal areas of focus: Activities of daily living } \\ \text { Evaluation of patient goal commitment: Not reported } \\ \text { Goal reminders used: Therapy involved regular discussion of goals with patients } \\ \text { Monitoring of progress towards goals: Goal progress was monitored in therapy and } \\ \text { exercises were altered as patients progressed }\end{array} \\ \begin{array}{l}\text { Power calculation: None reported } \\ \hline \text { Notes }\end{array}\end{array}$

Risk of bias

Bias Authors' judgement

Random sequence generation (selection Low risk bias)

Allocation concealment (selection bias) Low risk

Blinding of participants and personnel High risk (performance bias)

All outcomes

\section{Support for judgement}

'...participants were randomly allocated to one of the three treatment goal-setting conditions' (p.132). The random sequence was computer generated (author communication)

Allocation... 'was concealed as the [random sequence] list was kept on a computer and only consulted when a new participant was recruited. The physiotherapists had no idea which group participants would be allocated to before recruitment'

The intervention required active involvement of the patient and healthcare professionals, so blinding not possible 
Blinding of outcome assessment (detection High risk bias)

All outcomes

Incomplete outcome data (attrition bias) Unclear risk All outcomes
The healthcare providers and researcher, not blinded to group allocation, were involved in data collection

Of the 77 participants who originally enrolled in the study, eight $(10.4 \%)$ were withdrawn from the analysis because they did not complete their courses of physiotherapy. Of these eight, two were from the collaborative goal setting group, two were from the mandated goal setting group, and four were from the control group. 'All the participants who withdrew did so because they did not complete their course of physiotherapy and could not be tracked down' (author communication)

Unable to find a protocol for this study published prior to it being conducted, so unable to compare the outcomes reported with those planned to be measured at the outset

As each treating physiotherapist potentially provided all three types of intervention (collaborative goal setting, physiotherapist mandated goal setting, and no goal setting) the potential for cross-group contamination is high, particularly around the level of patient involvement in goal selection 
Funding: Department of Veterans Affairs Rehabilitation Research and Development Office

Recruitment (patients): Veterans were invited to participate in the study between January 1995 and March 1998

The method of recruitment was not reported

Recruitment (healthcare providers): Not reported

Inclusion criteria (patients): DSM-III-R diagnosis of schizophrenia or schizoaffective disorder, clinically stable, no housing changes, no psychiatric medication alterations or hospitalizations within 30 days prior to enrolment

Exclusions criteria (patients): neurological disease, developmental disability, traumatic brain injury. Substance abuse was explicitly stated to not be a reason for exclusio Consideration of people with comorbidities: Not reported

Participants

Interventions
Patients: 74 participants enrolled. 63 participants completed the study as determined by having completed sufficient work to have two biweekly evaluations of their work performance (100\% male). Mean age 44.4 (experimental group); 43.6 (control group) (SD 8.5 experimental group; 17.7 control group). 61.9\% Caucasian; 30.2\% AfricanAmerican; 7.9\% Hispanic

Principle health problems: DSM-III-R diagnoses of schizophrenia or schizoaffective disorder

Treatment currently receiving: A 26-week paid job placement working alongside fulltime employees at a Medical Center at an entry level position, with training, supervision and job coaching

Description of healthcare providers: Employees and supervisors at the work site trained the participants. A job coach was available at the participants' request. The professional characteristics of the people running the group performance review and goal setting session was not reported

Study aim: To test three hypotheses: 1) that a goal directed behavioural intervention (based on the WBI) would increase hours worked and weeks worked in people with schizophrenia participating in a work placement trial, 2) that a goal directed behavioural intervention would increase quality of life on interpersonal and intrapsychic dimensions, and 3) that these improvements in quality of life would be correlated with number of hours and weeks of work participation

Intervention: ( $\mathrm{n}=$ not reported, number completing intervention $=30$ ) Support work placement in an entry level unskilled position, with weekly feedback on work performance as measured by the WBI in a 60-minute small group meeting with peers (other study participants), followed by individualised goal setting for the next two-week period, focusing on goals that would improve WBI scores. Group discussions included problem solving around barriers to goal achievement. Graphical representations of WBI ratings were used to communicate this information. Participants documented their own goal on a time sheet which was kept at the workplace for recording daily work hours. When a goal was met, participants would set a new goal

Control: ( $\mathrm{n}=$ not reported, number completing intervention $=33$ ) Support work placement in an entry level unskilled position, without group WBI feedback and goal discussions

Delivery: Paid work placement over a 26-week period 
Fidelity: Evaluation of intervention fidelity not reported

Consumer involvement outside of the intervention: Not reported

Outcomes

Timing of outcomes: At baseline and on completion of the 26-week vocational placement programme

WBI - comprised of five subscales: 1. work habits, 2. work quality, 3. personal presentation, 4. co-operativeness, and 5. social skills, and a total score

Total hours and total weeks worked over the 26-week period of enrolment in the study Intrapsychic foundation and Interpersonal function subscales of the Quality of Life Scale

Goal setting characteristics

Comparison of interest: Collaborative goal setting plus strategies to enhance goal pursuit versus no goal setting

Patient involvement in goal setting: Goals collaboratively set by the patient and healthcare professional

Family involvement in goal setting: None reported

Name of goal setting approach: No specifically named approach used

Development of a plan for goal pursuit: Therapy included regular discussion of strategies to enhance goal achievement

Written copy of goals provided to patients: Clients self-recorded goals on their time sheet for recording daily work hours

Individual versus group-based goal discussion: Goal-based goal discussion with peers and a healthcare professional

Level of goal difficulty: Not reported

Goal areas of focus: All goals related to work performance

Evaluation of patient goal commitment: Not reported

Goal reminders used: Goals discussed in regular weekly group meetings

Monitoring of progress towards goals: Feedback on progress towards goals included data on work performance presented in a graph format for participants

Notes Power calculation: Not reported

Changes in study protocol: The original study protocol also involved randomising people to paid versus unpaid work, but it was reported that as 'most participants randomized to the unpaid condition declined to participate or did not sustain work activity very long' (p.45) these groups were excluded from analysis

Risk of bias

Bias

Random sequence generation (selection Low risk bias)
Authors' judgement

Support for judgement

'Randomization was done using a computerized number generator...' (author communication). Prior to randomisation the participants were stratified by two variables: a) whether or not they had past work experience; and b) whether or not they had prominent negative symptoms of schizophrenia or schizoaffective disorder (i. e. a score of 18 or greater on the Positive and Negative Syndrome Scale) 
Bell 2003

\begin{tabular}{|c|c|c|}
\hline Allocation concealment (selection bias) & Low risk & $\begin{array}{l}\text { Group allocation was managed by } \\
\text { 'creat [ing] sequential sealed envelopes with } \\
\text { subject ID sequentially on the outside and } \\
\text { randomization on the inside' (author com- } \\
\text { munication) }\end{array}$ \\
\hline
\end{tabular}

Blinding of participants and personnel High risk (performance bias)

All outcomes

Blinding of outcome assessment (detection High risk bias)

All outcomes
The intervention required active involvement of the patient and healthcare professionals, so blinding not possible

WBI Scores could not be blinded because feedback on these scores was conducted as part of the group-based intervention. Quality of Life Scale scores were singleblinded 'that is the subject's knew their condition but the rater did not' (author communication), however this outcome was self-reported by participants who were not blinded to group allocation

Incomplete outcome data (attrition bias) High risk All outcomes

'The imputation method of bringing last observation forward was employed for those participants whose last WBI came before the end of the 26-week active intervention phase' (p.47). However 11 of 74 participants (15\%) dropped out before the end of three weeks of work

Selective reporting (reporting bias) Unclear risk
Unable to find a protocol for this study published prior to it being conducted, so unable to compare the outcomes reported with those planned to be measured at the outset 
Setting: Three intermediate care nursing homes in a central Texas metropolitan area, USA

Funding: Not reported. Completed as a PhD thesis

Recruitment (patients): Participants were residents at the nursing home recruited into the study by the nursing directors in each home and the lead researcher

Recruitment (healthcare providers): Participating nursing homes recruited by ringing the 15 largest homes in the Texas metropolitan area to invite them to join the study, with all willing organisations being recruited. The day-shift staff members in each enrolled nursing home were then automatically recruited into the study

Inclusion criteria (patients): Residents of the enrolled nursing homes who required nursing staff to perform all morning self-care tasks on entry to study, having periodically done the tasks in the past without assistance, and who were thought to be physically capable of doing the tasks regularly by the nursing and medical staff; 'cognitively intact' based on general medical and nursing assessment

Exclusion criteria (patients): No further criteria reported

Consideration of people with comorbidities: Not reported 56 to 100 years. $82.0 \%$ white, Total $89=73$, White $(82.0 \%), 13$ Black $(14.6 \%), 3$ Hispanic (3.4\%). 79 participants completed the study

Principle health problems: Not reported

Treatment currently receiving: No treatment reported other than usual nursing home care support

Description of healthcare providers: Not reported

Study aim: To test the hypotheses that: 1) residents receiving mutual goal setting plus operant behaviour management (OBM) would perform significantly more self-care tasks than those receiving mutual goal setting only or those receiving usual care with no goal setting or OBM, and 2) residents receiving mutual goal setting without OBM would perform significantly more self-care tasks than those receiving usual care with no goal setting or OBM

Intervention (goal setting+OBM): $(\mathrm{n}=40)$ Goal setting plus OBM. All day-shift staff members were given two weeks of staff training on goal setting and OBM (one hour of training each day for two weeks; ten hours training total), with training covering: determinants of dependency, behavioural management of dependent behaviours, principles of OBM, strategies including prompting, shaping, positive reinforcement, resident involvement in treatment planning, development of treatment plans, preparation of goal attainment follow-up guides, and assessment of goal attainment. Following this training, a two-week period of baseline assessment was completed by the staff, at the end of which patients were asked to identify three areas of self-care behaviour that they wished to target. After this, staff members met with participating patients to set goals for this behavioural change. This was followed by a six-week period where the staff helped the patients improved their self-care behaviour, then a 16-week follow-up phase where staff helped patients maintain gains in their self-care behaviour. In addition, OBM strategies were used, which involved specific instructions for staff to say words of encouragement, and to give praise, smiles, affectionate touching, and engage in short conversations on topics of interest to patients when they made efforts towards desired behaviours Intervention (goal setting): $(\mathrm{n}=19)$ Mutual goal setting alone. As for the Intervention 
(goal setting+OMB) group, but staff were training for less time (one hour on two days per week for two weeks; four hours training total), and only given training in goal setting and goal evaluation. Residents were involved in goal setting and goal discussions after the two-week baseline assessment period, but OBM strategies were not used

Control: $(\mathrm{n}=30)$ As for the intervention groups but staff received less training (one hour per week for two weeks; two hours training total), with training only provided on followup goal attainment assessment, and no encouragement was given to staff to set goals with patients or to engage in behaviour change practices. After the two-week baseline assessment period, residents were still asked to identify three areas of self-care behaviours that they wished to target. After the two-week baseline assessment period, usual care was delivered to residents for 22 weeks without discussion of goals and without OBM Delivery: The assessments and interventions were delivered over a 24-week period Fidelity: A cluster-RCT approach was use to prevent cross-group contamination. This allowed the same training to be provided to all staff in each nursing home. However, 'no formal procedures were carried out to determine whether the staff incorporated the new information into their day-to-day work.' (p. 151)

Consumer involvement outside of the intervention: Not reported

Outcomes

Timing of outcomes: GAS data collected after the six weeks of skills requisition, and at eight and 16 weeks Rosenburg Self-esteem Scale data were collected

GAS - based on three individualised activities of daily living picked by patients and staff from a list (e.g. shaving, bathing, dressing, combing hair, feeding self, brushing teeth). GAS scores were taken from the care staffs' daily GAS records and not collected by an independent third party

Rosenburg Self-esteem Scale

Goal setting characteristics

Comparison of interest: Collaborative goal setting plus operant behaviour conditioning versus collaborative goal setting without operant behaviour conditioning versus usual care with no goal setting

Patient involvement in goal setting: Patients involved in goal selection, but these were selected from a list of six predetermined areas of self-care behaviour

Family involvement in goal setting: None reported

Name of goal setting approach: Goal Attainment Scaling

Development of a plan for goal pursuit: Goals informed development of a nursing care plan

Written copy of goals provided to patients: Not reported

Individual versus group-based goal discussion: Individual

Level of goal difficulty: The setting of achievable, realistic goals were emphasised in Blair 2001

Goal areas of focus: Activities of daily living

Evaluation of patient goal commitment: Not reported

Goal reminders used: Not reported

Monitoring of progress towards goals: Not reported

Notes

Power calculation: "A power analysis, using alpha of .05, power .80, and medium effect size .25 suggested the need for a sample size of 156 residents, 52 in each home" (p.161). However, only 89 residents were enrolled in the study and 79 were included in the data analysis

No adjustment reported for intraclass correlations arising from cluster randomisation 
Blair 1991 (Continued)

(randomisation at level of nursing home; analysis at level of participant)

\section{Risk of bias}

\begin{tabular}{|c|c|c|}
\hline Bias & Authors' judgement & Support for judgement \\
\hline $\begin{array}{l}\text { Random sequence generation (selection } \\
\text { bias) }\end{array}$ & Unclear risk & $\begin{array}{l}\text { 'Homes were randomly assigned to a re- } \\
\text { search condition' (Blair, 1995, p.161). In- } \\
\text { sufficient information provided about ran- } \\
\text { dom sequence generation }\end{array}$ \\
\hline Allocation concealment (selection bias) & Unclear risk & No information provided \\
\hline $\begin{array}{l}\text { Blinding of participants and personnel } \\
\text { (performance bias) } \\
\text { All outcomes }\end{array}$ & High risk & $\begin{array}{l}\text { The intervention required active involve- } \\
\text { ment of the patient and healthcare profes- } \\
\text { sionals, so blinding not possible }\end{array}$ \\
\hline $\begin{array}{l}\text { Blinding of outcome assessment (detection } \\
\text { bias) } \\
\text { All outcomes }\end{array}$ & High risk & $\begin{array}{l}\text { Outcome assessors not blinded to group al- } \\
\text { location }\end{array}$ \\
\hline $\begin{array}{l}\text { Incomplete outcome data (attrition bias) } \\
\text { All outcomes }\end{array}$ & Unclear risk & $\begin{array}{l}\text { Eleven per cent }(10 / 89) \text { of the original sam- } \\
\text { ple dropped out during the study before fol- } \\
\text { low-up assessment. Intention-to-treat anal- } \\
\text { ysis not reported }\end{array}$ \\
\hline Selective reporting (reporting bias) & Unclear risk & $\begin{array}{l}\text { Unable to find a protocol for this study } \\
\text { published prior to it being conducted, so } \\
\text { unable to compare the outcomes reported } \\
\text { with those planned to be measured at the } \\
\text { outset }\end{array}$ \\
\hline Other bias & Low risk & $\begin{array}{l}\text { Cross-group contamination managed by } \\
\text { cluster-RCT design. No evidence of other } \\
\text { major sources of bias }\end{array}$ \\
\hline
\end{tabular}


Setting: One privately owned intermediate-care nursing home in a metropolitan area of a Southern state in USA

Funding: Not reported

Recruitment (patients): Not reported

Recruitment (healthcare providers): Not reported

Inclusion criteria (patients): Residents of a nursing care home who were reliant on nursing staff to perform morning activities of daily living, judged by nurse and medical staff to be capable of carrying out their own activities of daily living, who were cognitively intact on basis of DSM criteria and Mini Mental State Exam scores > 23, and who were deemed able to participate in care planning and goal setting

Exclusion criteria (patients): No further criteria reported

Consideration of people with comorbidities: Not reported

Participants
Patients: 15 participants (26.7\% male). Age 78 (SD 10). Range 64 to 96 years. Ethnicity not stated

Principle health problems: Not reported

Treatment currently receiving: No treatment reported other than usual nursing home care support

Description of healthcare providers: Day-shift registered nurses and license practical nurses. All female. All with 2 to 4 years' experience. No staff attrition during the study

Interventions

Study aim: To compare the effectiveness of three nursing interventions: 1) OBM plus mutual goal setting, 2) mutual goal setting, 3) usual nursing care in terms of fostering the morning self-care behaviours of shaving, bathing, dressing, combing hair, feeding self, brushing teeth among nursing home residents

Intervention (goal setting+OBM): $(\mathrm{n}=5)$ All day-shift staff members were given two weeks of staff training on goal setting and OBM (one hour of training each day for two weeks; ten hours training total), with training covering: determinants of dependency, behavioural management of dependent behaviours, principles of OBM, strategies including prompting, shaping, positive reinforcement, resident involvement in treatment planning, development of treatment plans, preparation of goal attainment follow-up guides, and assessment of goal attainment. Following this training, a two-week period of baseline assessment was completed by the staff, at the end of which patients were asked to identify three areas of self-care behaviour that they wished to target. After this, staff members met with participating patients to set goals for this behavioural change. This was followed by a six-week period where the staff helped the patients improved their self-care behaviour, then a 16-week follow-up phase where staff helped patients maintain gains in their self-care behaviour. In addition, OBM strategies were used, which involved specific instructions for staff to say words of encouragement, and to give praise, smiles, affectionate touching, and engage in short conversations on topics of interest to patients when they made efforts towards desired behaviours

Intervention (goal setting): $(\mathrm{n}=5)$ Mutual goal setting alone. As for the Intervention (goal setting+OMB) group, but staff were training for less time (one hour on two days per week for two weeks; four hours training total), and only given training in goal setting and goal evaluation. Residents were involved in goal setting and goal discussions after the two-week baseline assessment period, but OBM strategies were not used

Control: $(n=5)$ As for the intervention groups but staff received less training (one hour per week for two weeks; two hours training total), with training only provided on follow- 
up goal attainment assessment, and no encouragement was given to staff to set goals with patients or to engage in behaviour change practices. After the two-week baseline assessment period, residents were still asked to identify three areas of self-care behaviours that they wished to target. After the two-week baseline assessment period, usual care was delivered to residents for 22 weeks without discussion of goals and without OBM

Delivery: The assessments and interventions were delivered over a 24-week period Fidelity: Application of training to practice was not formally evaluated. However all nursing staff were randomly assigned to just one of the three intervention conditions, which may have reduced cross-group contamination in this regard Consumer involvement outside of the intervention: Not reported

Outcomes

Timing of outcomes: Data collected after the six weeks of skills requisition, and at eight and 16 weeks

GAS - based on three individualised activities of daily living picked by patients and staff from a list (e.g. shaving, bathing, dressing, combing hair, feeding self, brushing teeth). GAS scores were taken from the care staffs' daily GAS records and not collected by an independent third party

Goal setting characteristics

Comparison of interest: Collaborative goal setting plus operant behaviour conditioning versus collaborative goal setting without operant behaviour conditioning versus usual care with no goal setting

Patient involvement in goal setting: Patients involved in goal selection, but these were selected from a list of six predetermined areas of self-care behaviour

Family involvement in goal setting: None reported

Name of goal setting approach: GAS

Development of a plan for goal pursuit: Goals informed development of a nursing care plan

Written copy of goals provided to patients: Not reported

Individual versus group-based goal discussion: Individual

Level of goal difficulty: Not reported

Goal areas of focus: Activities of daily living

Evaluation of patient goal commitment: Not reported

Goal reminders used: Not reported

Monitoring of progress towards goals: Not reported

Power calculation: Not reported

\section{Risk of bias}

\begin{tabular}{|c|c|c|}
\hline Bias & Authors' judgement & Support for judgement \\
\hline $\begin{array}{l}\text { Random sequence generation (selection } \\
\text { bias) }\end{array}$ & Unclear risk & $\begin{array}{l}\text { 'Residents and staff were randomly as- } \\
\text { signed to the three conditions' (p.1208) } \\
\text {. Insufficient information provided about } \\
\text { random sequence generation }\end{array}$ \\
\hline
\end{tabular}


Blair 1996 (Continued)

\begin{tabular}{|c|c|c|}
\hline $\begin{array}{l}\text { Blinding of participants and personnel } \\
\text { (performance bias) } \\
\text { All outcomes }\end{array}$ & High risk & $\begin{array}{l}\text { The intervention required active involve- } \\
\text { ment of the patient and healthcare profes- } \\
\text { sionals, so blinding not possible }\end{array}$ \\
\hline
\end{tabular}

\begin{tabular}{|c|c|c|}
\hline $\begin{array}{l}\text { Blinding of outcome assessment (detection } \\
\text { bias) } \\
\text { All outcomes }\end{array}$ & High risk & $\begin{array}{l}\text { Outcome assessors not blinded to group al- } \\
\text { location }\end{array}$ \\
\hline $\begin{array}{l}\text { Incomplete outcome data (attrition bias) } \\
\text { All outcomes }\end{array}$ & Low risk & $\begin{array}{l}\text { Outcome data collected on all participants } \\
\text { entering the study }\end{array}$ \\
\hline Selective reporting (reporting bias) & Unclear risk & $\begin{array}{l}\text { Unable to find a protocol for this study } \\
\text { published prior to it being conducted, so } \\
\text { unable to compare the outcomes reported } \\
\text { with those planned to be measured at the } \\
\text { outset }\end{array}$ \\
\hline Other bias & Unclear risk & $\begin{array}{l}\text { Potential exists for cross-group contamina- } \\
\text { tion }\end{array}$ \\
\hline
\end{tabular}

Cheng 2012

Methods

Goal setting and strategies to enhance goal pursuit for adults with acquired disability participating in rehabilitation (Review)

Copyright (c) 2015 The Cochrane Collaboration. Published by John Wiley \& Sons, Ltd.
Cluster-RCT. Participants clustered by community nurse service

Setting: Thirteen community nurse services based at six different hospitals, covering a wide region of Hong Kong

Funding: Not reported. Completed as a PhD thesis

Recruitment (patients): Recruited by a liaison nurse in each centre

Recruitment (healthcare providers): Department heads from the 13 community nurse services recruited the nurses for the study. Inclusion criteria: at least three years nursing experience, specialist training in community nursing, and expected to work at the service for the duration of the study

Training/support: Training was provided to all nurses participants in the study, and involved both classroom learning and community-based case examples. Knowledge and application of the training was formally evaluated through paper-based and home-based assignments. All nurses worked under the supervision of a senior ranked nurse (e.g. an Advanced Practice Nurse, Manager or Nursing Officer)

Inclusion criteria (patients): Participants needed to have had a chronic illness and be newly referred to community nursing; aged 18 or over; cognitively intact (able to express complaints, and discomfort)

Exclusion criteria (patients): living and receiving care in an institution; diagnosis of acute confusion, acute psychiatric illness, dementia, terminal illness such as end stage renal failure; referral for a one-off procedure such as blood-taking

Consideration of people with comorbidities: More the $70 \%$ of the enrolled patients had more than one active medical problem. Mean number of medical diagnoses was 2.4 (SD 1.3) 


\section{Cheng 2012 (Continued)}

Participants

Interventions
Patients: 96 participants (37.5\% male). Mean age 73 year (SD 11); 100\% Chinese Principle health problems: hypertension (46.9\%); diabetes mellitus (22.9\%); heart failure (20.8\%); cancer (17.7\%); osteoarthritis (11.5\%); chronic lung disease (10.4\%)

Treatment currently receiving: Community nursing

Description of healthcare providers: Thirteen community nurses (registered nurses and enrolled nurses, enrolled in a ratio of $4: 1$ )

Study aim: To test the hypotheses that patients with chronic health conditions receiving community nursing structured around mutual goal setting would have higher rates of goal achievement, better functional outcomes, better perceived health status, higher selfefficacy, and lower health service utilisation in comparison to a control group of patient receiving community nursing without mutual goal setting

Intervention: ( $\mathrm{n}=53$ ) Routine community nursing plus seven sessions of mutual goal setting over eight weeks. Goals were collaboratively developed with each patient, starting with a pre-determined list of common goals, with the option of adding new goals if required. GAS scales were developed for all goals, with an expert panel of nurses involved in the development of any new scales. A goal setting record was documented and signed by both the nurse and patient, and reviewed in follow up meetings

Control: ( $\mathrm{n}=43$ ) Routine community nursing without mutual goal setting. Care goals were documented in the Community Based Nursing Service information system, but were not established through a process of mutual goal setting with each patient (i.e. these goals were set by nurses, not discussed with patients, and just used to document an expected outcome arising as part of the care plan)

Delivery: Mutual goal setting was delivered over eight-week. The duration of delivery of the routine nursing care was not reported

Fidelity: All nurse participants worked under the supervision of a senior ranked nurse. Delivery of the intervention was monitored by observation and audit of the study records Consumer involvement outside of the intervention: None reported

Outcomes

Timing of outcomes: Four, 12, and 24 weeks after baseline data collection

Goal achievement (percentage of goals achieved)

Chronic Disease Self-efficacy Scale

Disability Index of the Health Assessment Questionnaire

Perceived health status, measured using the World Organization of National Colleges, Academics, and Academic Associations of General Practices/Family Physicians Charts

Satisfaction Scale in Community Nursing

Number of emergency department visits

Days of hospitalisation

Mortality

Hospital readmissions

Goal setting characteristics
Comparison of interest: Collaborative goal setting plus strategies to enhance goal pursuit versus usual care with no structured or required approach to goal setting

Patient involvement in goal setting: Goals collaboratively set by the patient and healthcare professional

Family involvement in goal setting: None reported

Name of goal setting approach: GAS within the context of King's theory of goal attainment (King 1981)

Development of a plan for goal pursuit: A plan for goal pursuit was collaboratively 
Cheng 2012 (Continued)

developed by both the nurse and patient

Written copy of goals provided to patients: Not reported

Level of goal difficulty: The setting of realistic goals was emphasised

Goal areas of focus: Mixed, including activity limitations, participation restrictions, body

structure and function, health knowledge, health behaviour, psychological state

Notes

Risk of bias

\begin{tabular}{l|l|l}
\hline Bias & Authors' judgement & Support for judgement \\
\hline $\begin{array}{l}\text { Random sequence generation (selection } \\
\text { bias) }\end{array}$ & Low risk & $\begin{array}{l}\text { The randomisation sequence was generated } \\
\text { 'using computerized software (System Ran- } \\
\text { domizer)' (p. } 87 \text { ) }\end{array}$ \\
\hline Allocation concealment (selection bias) & High risk & $\begin{array}{l}\text { Concealment of group allocation during } \\
\text { patient recruitment was not possible due to } \\
\text { the use of cluster randomisation. Recruit- } \\
\text { ment of patients was undertaken by a liai- } \\
\text { son nurse in each centre who would have } \\
\text { known whether the centre was in the exper- } \\
\text { imental or control group, therefore which } \\
\text { group the patients were going into }\end{array}$ \\
\hline
\end{tabular}

Blinding of participants and personnel High risk (performance bias)

The intervention required active involveAll outcomes ment of the patient and healthcare professionals, so blinding not possible

Blinding of outcome assessment (detection Unclear risk bias)

'The raters had no knowledge as to which study group the patients belonged' (p. 141) . However, a number of the outcomes were self-reported by participants who were not blinded to group allocation

Incomplete outcome data (attrition bias) High risk All outcomes

Outcome data were not collected for $28 \%$ (27/96) of the sample population. For $18 \%$ of the study population this was due to death or hospitalisation before the 24-week assessment point. 'Findings of this study were analyzed by only those who completed the study at the 24-week follow-up' (p. 157)

Selective reporting (reporting bias) Unclear risk

Unable to find a protocol for this study published prior to it being conducted, so unable to compare the outcomes reported with those planned to be measured at the outset

Goal setting and strategies to enhance goal pursuit for adults with acquired disability participating in rehabilitation (Review) 
Cheng 2012 (Continued)

Other bias L Low risk

Risk of cross-group contamination low due to use of cluster-RCT methods. No evidence of other sources of bias

Conrad 2000

Methods

RCT

Setting: A cardiac rehabilitation service in Halifax, Nova Scotia

Funding: Not reported

Recruitment (patients): Patients recruited from a voluntary cardiac rehabilitation programme

Recruitment (healthcare providers): Not reported

Inclusion criteria (patients): Established coronary artery disease

Exclusion criteria (patients): A baseline diet (based on 4-day assessment) containing less than $20 \%$ fat energy

Consideration of people with comorbidities: Not reported

Participants

Patients: 7 participants. No demographic data reported

Principle health problems: Coronary artery disease including stable angina, pervious myocardial infarction, or coronary artery bypass

Treatment currently receiving: Cardiac rehabilitation

Description of healthcare providers: The dietician providing the meal plans was one of the researchers

Interventions

Study aim: To compare the effectiveness of a goal of incrementally decreasing fat intake to a targeted maximum of $10 \%$ of total energy versus the effectiveness of a goal to immediately decreased fat intake to $10 \%$ of total energy

All participants and their significant others attended the same cardiac rehabilitation group (eight weeks of health education), followed by a four-day non-consecutive food record, and participated in the setting of individual meal plans with a dietician

Intervention: $(\mathrm{n}=4)$ Incremental reduction in fat intake. Meal plans targeted incremental reduction: $26-30 \%$ fat energy for the first month, $20 \%$ fat energy for the second month, $10 \%$ fat energy for the fourth month

Control: $(n=3)$ Immediate reduction in fat intake. Meal plans targeted immediate reduction in fat intake to $10 \%$ of total energy, with these plans being reinforced two and four months later

Delivery: Meal plans and reinforcement of plans delivered over a four-month period

Fidelity: Not reported, but all interventions were delivered by one dietician

Consumer involvement outside of the intervention: Not reported

Outcomes

Timing of outcomes: Within one week following each meeting with the dietician (i.e. during months $0,2,4)$ and at 7 months

Percentage of energy from dietary fat, carbohydrate, protein based on unannounced 24hour diet recall administered by a trained research assistant over phone Milligrams of cholesterol

Body weight

Goal setting and strategies to enhance goal pursuit for adults with acquired disability participating in rehabilitation (Review) 
Conrad 2000 (Continued)

Goal setting characteristics

Comparison of interest: Setting of an end goal for immediate achievement versus setting of incremental steps towards end goal achievement

Patient involvement in goal setting: None. Goals prescribed according to group allocation Family involvement in goal setting: None

Name of goal setting approach: No specifically named approach used

Development of a plan for goal pursuit: Education and individual meal plans provided to patients and family members targeting strategies for goal achievement

Written copy of goals provided to patients: Meal plans include documentation of therapy goals

Individual versus group-based goal discussion: Individual

Level of goal difficulty: The study involved comparison of two types of goal difficulty

Goal areas of focus: Dietary behaviour

Evaluation of patient goal commitment: Not reported

Goal reminders used: Meal plans were reinforced two and four months after the plan was established

Monitoring of progress towards goals: Not reported

Power calculation: Not reported

Risk of bias

Bias

Random sequence generation (selection Low risk bias)
Authors' judgement

Support for judgement

A randomized, controlled repeated measure design was used...' (p. 194) The random sequence was generated by placing the names of participants in a container and, in the presence of a co-worker, having a dietitian randomly choosing from the container for participants to be in either the control or experimental group (author communication)

Allocation concealment (selection bias) Unclear risk

The dietitian drawing the names of the participants for each group was the same person who was to deliver the intervention, but this was conducted in the presence of a third-party, reducing risk of selection bias (author communication)

The intervention required active involvement of the patient and healthcare professionals, so blinding not possible

(performance bias)

All outcomes

Blinding of outcome assessment (detection High risk bias)

All outcomes
Outcomes related to dietary intake were entirely based on patient self-report, who were not blinded to group allocation 
Conrad 2000 (Continued)

\begin{tabular}{l|l|l}
\hline $\begin{array}{l}\text { Incomplete outcome data (attrition bias) } \\
\text { All outcomes }\end{array}$ & Low risk & No reported attrition of participants \\
\hline Selective reporting (reporting bias) & Unclear risk & $\begin{array}{l}\text { Unable to find a protocol for this study } \\
\text { published prior to it being conducted, so } \\
\text { unable to compare the outcomes reported } \\
\text { with those planned to be measured at the } \\
\text { outset }\end{array}$ \\
\hline Other bias & Low risk & No evidence of other sources of bias \\
\hline
\end{tabular}

Coote 2012

$\begin{array}{ll}\text { Rethods } & \text { RCT } \\ \text { Setting: Community, UK } \\ \text { Funding: Not reported } \\ \text { Recruitment (patients): Patients were recruited, by mail, from via a registered charity } \\ \text { (Depression UK) } \\ \text { Recruitment (healthcare providers): The intervention was delivered by mail, with no } \\ \text { healthcare providers being involved and minimal contact with the researchers } \\ \text { Training/support: n/a } \\ \text { Inclusion criteria (patients): gave consent for the researcher to notify their general prac- } \\ \text { titioner of their involvement in the study; scored } 16 \text { or above on the Centre for Epi- } \\ \text { demiological Studies-Depression scale } \\ \text { Exclusion criteria (patients): None reported } \\ \text { Consideration of people with comorbidities: Not reported }\end{array}$

Participants

Patients: 64 participants enrolled in the study, 55 completed the study intervention (29. $1 \%$ male). Mean age 53.3 (experimental group); 51.8 years (control group) (SD 14.8 experimental group; 11.9 control group). $92.7 \%$ White; $7.3 \%$ Other

Principle health problems: Depression

Treatment currently receiving: $63.6 \%$ were on antidepressants; $20 \%$ were currently in therapy

Description of healthcare providers: $\mathrm{n} / \mathrm{a}$

Study aim: To test the hypothesis that a self-directed GAP for people with depression would result in significant improvements in general well-being and reduction in depressive symptoms

Intervention: $(\mathrm{n}=30)$ The GAP programme. Participants were sent the GAP manual to work through individually over 5 weeks. They received a brief telephone call from the researcher at the end of the second week to monitor adherence and progress and provide minimal support with identifying appropriate goals. General counselling was avoided as part of the study intervention. The GAP manual consisted of three parts: 1) the concept of well-being, goals and plans; including tasks to think of self-concordant goals and how to achieve them, using the worksheets provided within the manual, 2) review of goal progress and benefits noted; identifying obstacles to achieving goals and solutions for overcoming them, 3) final week overview of the previous information; including how to maintain progress. The manual focused on life goals, and minimised reference to 
Coote 2012 (Continued)

\begin{tabular}{|c|c|}
\hline & $\begin{array}{l}\text { symptoms of depression } \\
\text { Control: }(\mathrm{n}=34) \text { Wait list control } \\
\text { Delivery: By post, over a 5-week period } \\
\text { Fidelity: Not reported } \\
\text { Consumer involvement outside of the intervention: Not reported }\end{array}$ \\
\hline Outcomes & $\begin{array}{l}\text { Timing of outcomes: } 5 \text { weeks after delivery of GAP manual } \\
\text { Positive and Negative Affect Scale } \\
\text { Satisfaction with Life Scale } \\
\text { Centre for Epidemiological Studies-Depression Scale }\end{array}$ \\
\hline Goal setting characteristics & $\begin{array}{l}\text { Comparison of interest: Goal setting plus strategies to enhance goal pursuit versus no } \\
\text { goal setting } \\
\text { Patient involvement in goal setting: Largely patient directed goal selection and planning } \\
\text { Family involvement in goal setting: None reported } \\
\text { Name of goal setting approach: GAP programme } \\
\text { Development of a plan for goal pursuit: The GAP manual involved a section directing } \\
\text { the participants to develop a plan for goal achievement } \\
\text { Written copy of goals provided to patients: All goal documents were retained by the } \\
\text { patients } \\
\text { Level of goal difficulty: Not reported } \\
\text { Goal areas of focus: Not reported } \\
\text { Evaluation of patient goal commitment: Not reported } \\
\text { Goal reminders used: One brief telephone call from the researchers at the end of the } \\
\text { second week of the intervention period was used to monitor adherence and progress with } \\
\text { the GAP programme } \\
\text { Monitoring of progress towards goals: The GAP manual included instruction for self- } \\
\text { monitoring of progress towards goals }\end{array}$ \\
\hline Notes & \\
\hline & \\
\hline
\end{tabular}

Risk of bias

Bias

Random sequence generation (selection High risk bias)

Allocation concealment (selection bias) High risk

Blinding of participants and personnel High risk (performance bias)

All outcomes

\section{Support for judgement}

Participants were alternately allocated to treatment or control groups after enrolment in the study (author communication)

Participants were alternately allocated to treatment or control groups after enrolment in the study (author communication)

The intervention required active involvement of the patient and healthcare professionals, so blinding not possible 
Coote 2012 (Continued)

\begin{tabular}{|c|c|c|}
\hline $\begin{array}{l}\text { Blinding of outcome assessment (detection } \\
\text { bias) } \\
\text { All outcomes }\end{array}$ & High risk & $\begin{array}{l}\text { All outcome measures self-reported by pa- } \\
\text { tients who would have known the details } \\
\text { of the intervention that they received, be- } \\
\text { cause the intervention required their active } \\
\text { involvement }\end{array}$ \\
\hline
\end{tabular}

Incomplete outcome data (attrition bias) Unclear risk

All outcomes

$14 \%$ drop-out of participants before data collection. Intention-to-treat analysis not employed

Selective reporting (reporting bias) Unclear risk

Unable to find a protocol for this study published prior to it being conducted, so unable to compare the outcomes reported with those planned to be measured at the outset

\begin{tabular}{l|l} 
Other bias & Low risk $\quad$ No evidence of other sources of bias
\end{tabular}

\section{Coppack 2012}

Methods

RCT

Setting: Inpatient unit for the UK Defence Medical Rehabilitation Centre (DMRC), Surrey, UK

Funding: 'This research received no specific grant from any funding agency in the public, commercial, or not-for-profit sectors' (p. 1041)

Recruitment (patients): Consecutive admissions to the DMRC inpatient unit

Recruitment (healthcare providers): Three specialist exercise therapists with a mean of six years' experience were recruited to provide the exercise programmes. The exercise therapists were randomly allocated to each of the trial groups; one to each group Training/support: Not reported

Inclusion criteria (patients): Patients admitted to the early spines treatment group at the DMRC for chronic low back pain

Exclusion criteria (patients): None reported

Consideration of people with comorbidities: Not reported

Participants

Patients: 48 participants (93.8\% male). Average age 33 years (SD 8). Ethnicity not reported

Principle health problems: Chronic low back pain (mean duration 2.6 years; SD 0.3)

Treatment currently receiving: Residential rehabilitation over 3 weeks, 5 days per week, 15-day total intervention

Description of healthcare providers: 'Three specialist exercise therapists with a mean of six years' experience supervised treatment sessions' (p.1034)

Interventions

Study aim: To test the hypothesis that structured exercise plus goal setting for people with chronic low back pain would resulting in significantly higher self-efficacy, treatment efficacy, adherence to treatment, and treatment outcomes when compared to structured exercise without goal setting

Intervention: $(\mathrm{n}=16)$. Therapist-directed exercise with collaborative goal setting. The

Goal setting and strategies to enhance goal pursuit for adults with acquired disability participating in rehabilitation (Review) 


\section{Coppack 2012 (Continued)}

standardised exercise therapy consisted of ten 30-minute exercise sessions per day for 15 days, with individual and group-based submaximal, incremental exercise, which included spinal mobility, muscle strength, stretching, co-ordination, and low-intensity cardiovascular conditioning. Prior to beginning the exercise programme, participants negotiated therapy goals with the researcher. Goals were scored for perceived importance and for performance levels at base line. These scores were used to calculate the treatment goal priorities. Goals were reviewed and revised on Day 6 and Day 11 of the programme. All exercise sessions were supervised by a specialist exercise therapist

Control 1: $(\mathrm{n}=16)$. Therapist-directed exercise without goal setting. An identical exercise programme was delivered for the same duration and intensity, with supervision by a specialist exercise therapist, but without any goal setting

Control 2: $(\mathrm{n}=16)$. Non-therapist-directed exercise without goal setting. An identical exercise programme was delivered for the same duration and intensity, but without direction provided by an exercise therapist and without any goal setting. A specialist exercise therapist was present for the exercise sessions to ensure safety, but provided no verbal encouragement or discussion of exercise regime

Delivery: All therapists delivered over 15 days, across three weeks

Fidelity: Not reported

Consumer involvement outside of the intervention: None reported

Outcomes

Timing of outcomes: Collected at the end of the 15-day exercise programme Sports Injury Rehabilitation Adherence Scale Sports Injury Rehabilitation Beliefs Survey

Biering-Sørensen test

Goal setting characteristics

Comparison of interest: Goal setting with or without strategies to enhance goal pursuit versus no goal setting

Patient involvement in goal setting: Patients were involved in the selection and prioritisation of goals

Family involvement in goal setting: None reported

Name of goal setting approach: The goal setting intervention was based on Personal Construct Theory, but not specifically named approach to goal setting was used

Development of a plan for goal pursuit: Goal achievement was explicitly linked to the prescribed exercise programme

Written copy of goals provided to patients: Not reported

Level of goal difficulty: Not reported

Goal areas of focus: Mixed

Evaluation of patient goal commitment: Not reported

Goal reminders used: Goal discussed with researchers on Day 6 and Day 11 of the 15day programme

Monitoring of progress towards goals: Progress towards goals discussed with researchers on Day 6 and Day 11 of the 15-day programme

Notes

Risk of bias

Bias

Authors' judgement

Support for judgement

Goal setting and strategies to enhance goal pursuit for adults with acquired disability participating in rehabilitation (Review)

Copyright @ 2015 The Cochrane Collaboration. Published by John Wiley \& Sons, Ltd. 


\section{Coppack 2012 (Continued)}

\begin{tabular}{|c|c|c|}
\hline $\begin{array}{l}\text { Random sequence generation (selection } \\
\text { bias) }\end{array}$ & Unclear risk & $\begin{array}{l}\text { Insufficient information provided to deter- } \\
\text { mine whether adequate sequence genera- } \\
\text { tion }\end{array}$ \\
\hline Allocation concealment (selection bias) & Unclear risk & No information provided \\
\hline $\begin{array}{l}\text { Blinding of participants and personnel } \\
\text { (performance bias) } \\
\text { All outcomes }\end{array}$ & Unclear risk & $\begin{array}{l}\text { 'To avoid possible contamination, subjects } \\
\text { were advised that the purpose of the study } \\
\text { was to examine the effects of injury on pa- } \\
\text { tients' responses to residential rehabilita- } \\
\text { tion. Subjects were unaware of the experi- } \\
\text { mental and control conditions employed in } \\
\text { the study.' (p. 1034) However, it would not } \\
\text { have been possible to blind the healthcare } \\
\text { professionals who were involved in deliver- } \\
\text { ing the intervention to group allocation }\end{array}$ \\
\hline
\end{tabular}

Blinding of outcome assessment (detection Low risk bias)

All outcomes

'Two independent therapists, who were blind to the subjects' group assignment, rated the participants' (p.1034)

Incomplete outcome data (attrition bias) Low risk

No reported attrition of participants

All outcomes

Selective reporting (reporting bias) Unclear risk

Unable to find a protocol for this study published prior to it being conducted, so unable to compare the outcomes reported with those planned to be measured at the outset 
Exclusion criteria (patients): medical contraindication for the addition of cottage cheese, fresh fruit or orange juice to the diet; inability to speak or write in English; diagnosis of emotional or psychological disturbance; blind or deaf; under 21 years; uncomfortable or in pain; or being unable to mark a menu selection card independently

Consideration of people with comorbidities: Not reported hospitalisation will result in a positive health behaviour change, and that 2) patients who are directed toward meeting short-term and immediate goals established in the health teaching/learning situation will show a greater health behaviour change than will patients who are not directed toward a goal in the same learning situation

Intervention (Dietary education): $(\mathrm{n}=15)$ Nurse delivered education on the importance of protein and vitamin $\mathrm{C}$ for healing

Intervention (Dietary education plus goal setting): $(\mathrm{n}=15)$ Dietary education with goal setting. Nurse delivered education as above, plus three short-term prescribed goals: 1) to choose one cup of orange juice per day, 2) to choose cottage cheese twice each day, and 3) to choose fresh fruit twice each day. Goals were discussed verbally, and not written down

Control: $(n=15)$ Usual care, with regular nursing staff instructed not to provide dietary advice during the period of the study

Delivery: Timing and duration of the study not reported

Fidelity: Not reported

Consumer involvement outside of the intervention: No foods were selected/times food offered). Data three days after teaching were compared to baseline frequency of desirable food selection based on average across two days prior to dietary education. Food selection scores were averaged across two days at baseline. Data collected from the participants' menu selection cards, scored by the researchers input

Patient involvement in goal setting: Goals prescribed by healthcare professionals Family involvement in goal setting: None 


\begin{tabular}{ll} 
& Name of goal setting approach: No specifically named approach used \\
Development of a plan for goal pursuit: Plan prescribed by healthcare professionals \\
Written copy of goals provided to patients: Not reported \\
Individual versus group-based goal discussion: Individual \\
Level of goal difficulty: Goal difficulty was not considered as part of the study \\
Goal areas of focus: Dietary behaviour \\
Evaluation of patient goal commitment: Not reported \\
Goal reminders used: None \\
Monitoring of progress towards goals: None \\
\hline Notes & Power calculation: Not reported
\end{tabular}

\section{Risk of bias}

\begin{tabular}{|c|c|c|}
\hline Bias & Authors' judgement & Support for judgement \\
\hline $\begin{array}{l}\text { Random sequence generation (selection } \\
\text { bias) }\end{array}$ & Unclear risk & $\begin{array}{l}\text { 'The selected patients were randomly as- } \\
\text { signed to one of the following three groups. } \\
. . \text { (p. } 455) \text {. Insufficient information pro- } \\
\text { vided to determine whether adequate se- } \\
\text { quence generation }\end{array}$ \\
\hline Allocation concealment (selection bias) & Unclear risk & No information provided \\
\hline $\begin{array}{l}\text { Blinding of participants and personnel } \\
\text { (performance bias) } \\
\text { All outcomes }\end{array}$ & High risk & $\begin{array}{l}\text { The healthcare professional providing the } \\
\text { intervention would not have been blinded } \\
\text { to group allocation. The participants may } \\
\text { or may not have been blinded to the na- } \\
\text { ture of the intervention, but were required } \\
\text { to be active involved in the delivery of the } \\
\text { intervention }\end{array}$ \\
\hline $\begin{array}{l}\text { Blinding of outcome assessment (detection } \\
\text { bias) } \\
\text { All outcomes }\end{array}$ & Unclear risk & $\begin{array}{l}\text { It is not reported whether the participants } \\
\text { knew that their menu selections would be } \\
\text { used as data in the study }\end{array}$ \\
\hline $\begin{array}{l}\text { Incomplete outcome data (attrition bias) } \\
\text { All outcomes }\end{array}$ & Low risk & No reported attrition of participants \\
\hline Selective reporting (reporting bias) & Unclear risk & $\begin{array}{l}\text { Unadjusted SDs for the main outcomes are } \\
\text { not available. Authors reported some statis- } \\
\text { tically significant findings, but appeared to } \\
\text { have adjusted their analysis (e.g. combin- } \\
\text { ing groups; reporting change scores when } \\
\text { end scores were not significant) in order to } \\
\text { find these }\end{array}$ \\
\hline Other bias & Low risk & No evidence of other sources of bias \\
\hline
\end{tabular}

Goal setting and strategies to enhance goal pursuit for adults with acquired disability participating in rehabilitation (Review) 


\begin{tabular}{ll} 
Methods & RCT \\
Setting: Two post-acute brain injury rehabilitation centres, Scotland \\
Funding: NHS Education for Scotland and the Sacklet Foundation \\
Recruitment (patients): Possible participants identified by the clinical service managers \\
in each centre and sent a letter of invitation \\
Recruitment (healthcare providers): Not reported \\
Inclusion criteria (patients): documented acquired brain injury; memory impairment \\
(based on neuropsychological assessment or clinical judgement); actively participating \\
in rehabilitation; at least three months post-injury; owned a mobile phone; had at least \\
six therapy goals set as part of their rehabilitation programme \\
Exclusion criteria (patients): severe receptive or expressive language difficulties; unable \\
to reliably access text messages on their phone; significant difficulties with aggression; \\
consistent failure to engage in the rehabilitation programmes; under the Adults with \\
Incapacity (Scotland) Act; or not able to provide informed consent \\
Consideration of people with comorbidities: Not reported \\
\hline Participants & $\begin{array}{l}\text { Pthnicity not reported } 11 \text { participants (72.7\% male). Mean age } 36 \text { (SD 14). Range } 18 \text { to } 60 \text { years. } \\
\text { Principle health problems: Acquired brain injury (severe to very severe brain injury based } \\
\text { on coma duration or length of posttraumatic amnesia) } \\
\text { Treatment currently receiving: Inpatient or outpatient rehabilitation for brain injury } \\
\text { Description of healthcare providers: Not stated }\end{array}$ \\
\hline
\end{tabular}
phones of patients with brain injury would result in significantly better recall of their rehabilitation goals in comparison to not providing text message reminders about their goals

Intervention: ( $\mathrm{n}=33$ goals) Text message reminders of rehabilitation goals. If participants had more than six goals, then six were selected at random. Each goal was expressed as a single sentence that the participant could understand. Participants were then involved in setting 'cue words' for each of the six goals - that would help cue recall. Seven days later baseline recall of the six rehabilitation goals was assessed. Three goals were then selected at random to the text message reminder condition. Text messages for these goals were then sent via an online text messaging service three times per day for 14 days Control: ( $\mathrm{n}=33$ goals) As above, but no text messages on these goal sent

Delivery: Goal setting, then baseline assessment for one week, followed by text messages being sent over a two-week period

Fidelity: Not reported

Consumer involvement outside of the intervention: Not reported

Outcomes

Goal setting characteristics
Timing of outcomes: Seven and 14 days after baseline assessment of goal recall Recall of goals score on a four-point scale. Two types of recall examined: 1 . free recall (based on open-ended question re. the participant's goals) and cued recall (seeing if the participant can remember their goals based on their negotiated 'cue words')

Comparison of interest: Enhanced approach to improving goal recall versus usual care Patient involvement in goal setting: Not reported

Family involvement in goal setting: None reported

Name of goal setting approach: No specifically named approach used 
Development of a plan for goal pursuit: Not the subject of the research

Written copy of goals provided to patients: Usual practice around documentation of goals not reported

Individual versus group-based goal discussion: Individual

Level of goal difficulty: Not reported

Goal areas of focus: Not reported

Evaluation of patient goal commitment: Not reported

Goal reminders used: The study involved comparison of text messaging reminders of goals versus no additional goal reminders

Monitoring of progress towards goals: Not the subject of the research

Notes Power calculation: Not reported

\section{Risk of bias}

\begin{tabular}{l|l} 
Bias & Authors' judgement
\end{tabular}

Random sequence generation (selection Low risk bias)
'Six indistinguishable envelopes containing the numbers one to six were shuffled and the participant was asked to select three envelopes. The three selected goals were then entered onto an online text messaging service called textanywhere.net' (p. 109)

See above

\begin{tabular}{ll} 
Allocation concealment (selection bias) & Low risk \\
\hline $\begin{array}{l}\text { Blinding of participants and personnel } \\
\text { (performance bias) }\end{array}$ & High risk
\end{tabular}

All outcomes

bias)

All outcomes

Blinding of outcome assessment (detection Unclear risk

All outcomes

Selective reporting (reporting bias) Unclear risk

Unable to find a protocol for this study published prior to it being conducted, so unable to compare the outcomes reported with those planned to be measured at the outset 
Culley 2010 (Continued)

\begin{tabular}{l|l} 
Other bias $\quad$ Low risk $\quad$ No evidence of other sources of bias
\end{tabular}

Duncan 2003

Methods

Participants
RCT

Setting: A heart failure clinic in Nebraska, USA

Funding: American Heart Association and University of Negraska Medical Centre

Recruitment (patients): Recruited from current patients in a heart failure clinic

Recruitment (healthcare providers): Not reported

Inclusion criteria (patients): Cardiac ejection fraction < (or =) 40\%; receiving standard pharmacologic therapy (beta-blockers, angiotension-converting enzymes, digoxin, and diuretics); permission to attend from cardiologist; able to attend an exercise class three times per week at the cardiac rehabilitation facility; had not participated in supervised exercise programme within 30 days before the study

Exclusion criteria (patients): No further criteria reported

Consideration of people with comorbidities: Not reported

Patients: 16 participants enrolled. 14 participants complete ( $85.7 \%$ male). Mean age 66 (SD not reported). Ethnicity not reported

Principle health problems: Heart failure (64.3\% with Ischaemic cardiac disease)

Treatment currently receiving: Cardiac rehabilitation

Description of healthcare providers: Not reported

Interventions
Study aim: To test the effectiveness of an intervention (goal setting, graphic feedback, and problem solving) on physiological outcomes, functional outcomes, and quality of life in a sample of patients with heart failure who completed a two-phase exercise programme All participants received a two-phase exercise programme, with Phase 1 being a 12week supervised programme and Phase 2 being a 12-week unsupervised home based programme. Phase 1 was run from a cardiac rehabilitation facility and involved a three times weekly, structured 60 minute exercise programme, plus encouragement to do aerobic exercises two to three times a week at home

Intervention: $(\mathrm{n}=8)$ Exercise with adherence facilitation. During Phase 1 of the exercise programme, the cardiac rehabilitation staff set weekly exercise goals for frequency (number of sessions/weeks) and duration (number of aerobic minutes per session). Bar graphs were created for each patient comparing their weekly exercise participation to their exercise goals. These graphs were shared with the participants in an intervention group at 3-week intervals throughout the 12 weeks of supervised exercise, with positive reinforcement for goal achievement or problem solving discussions if goal has not been met. '... goal were adjusted [i.e. made easier] if warranted by the patient's medical condition' (p. 119). After Phase 1, the cardiac rehabilitation staff set individual patient goals for Phase 2 for exercise frequency and duration, recording these in the patients' diaries. The participants recorded all exercise sessions completed during Phase 2 in the exercise diary. During the 12 weeks of unsupervised exercise, participants mailed their exercise diaries to the nurse every three weeks; and graphs of goal progress were mailed back, plus these participants received follow up phone calls with the cardiac rehabilitation staff to review their progress

Control: $(\mathrm{n}=8)$ Exercise without adherence facilitation. As for the intervention group, but without the cardiac rehabilitation staff sharing or discussing goals or goal progress 
Duncan 2003 (Continued)

with the patients

Delivery: The intervention was delivered over a 24-week period of exercise

Fidelity: Not reported

Consumer involvement outside of the intervention: Not reported

\begin{tabular}{|c|c|}
\hline Outcomes & $\begin{array}{l}\text { Timing of outcomes: At baseline, } 12 \text { weeks and } 24 \text { weeks } \\
\text { VO2 max } \\
\text { Baseline Dyspnea Index } \\
\text { Piper Fatigue Scale } \\
\text { 6-Minute Walk Test } \\
\text { Minnesota Living With Heart Failure } \\
\text { Number of prescribed home exercise sessions completed, self-reported }\end{array}$ \\
\hline Goal setting characteristics & $\begin{array}{l}\text { Comparison of interest: Prescribed goal setting plus strategies to enhance goal pursuit } \\
\text { versus no goal setting } \\
\text { Patient involvement in goal setting: All goals were prescribed by healthcare professionals } \\
\text { for patients } \\
\text { Family involvement in goal setting: None } \\
\text { Name of goal setting approach: No specifically named approach used } \\
\text { Development of a plan for goal pursuit: All goals related to a prescribed exercise pro- } \\
\text { gramme } \\
\text { Written copy of goals provided to patients: Goals documented in the patients exercise } \\
\text { diaries } \\
\text { Individual versus group-based goal discussion: Individual } \\
\text { Level of goal difficulty: Not reported } \\
\text { Goal areas of focus: Exercise frequency and duration } \\
\text { Evaluation of patient goal commitment: Not reported } \\
\text { Goal reminders used: Prompting regarding goals was incorporate in the feedback on } \\
\text { progress to goal achievement } \\
\text { Monitoring of progress towards goals: Weekly progress toward goal achievement was } \\
\text { presented to patients in graphical form }\end{array}$ \\
\hline Notes & Power calculation: Not reported \\
\hline
\end{tabular}

Risk of bias

\begin{tabular}{|c|}
\hline Bias \\
\hline
\end{tabular}

Random sequence generation (selection Low risk bias)

Participants 'were randomly assigned to either the exercise-only group (control) group or the exercise-with-adherence facilitation group (intervention)' (Duncan 2003, p. 118). Assignment was by drawing lots (authors communication)

Allocation concealment (selection bias) Unclear risk

Insufficient information provided

Goal setting and strategies to enhance goal pursuit for adults with acquired disability participating in rehabilitation (Review) 
Duncan 2003 (Continued)

\begin{tabular}{l|l|l}
$\begin{array}{l}\text { Blinding of participants and personnel } \\
\text { (performance bias) } \\
\text { All outcomes }\end{array}$ & $\begin{array}{l}\text { The intervention required active involve- } \\
\text { ment of the patient and healthcare profes- } \\
\text { sionals, so blinding not possible }\end{array}$
\end{tabular}

Blinding of outcome assessment (detection Unclear risk bias)

All outcomes

The researchers collecting the outcome data were not involved in delivery of the interventions and were blinded to group allocation (author communication). However, a number of the outcome measures used were based on self-report by participants who were not blinded to group allocation

Incomplete outcome data (attrition bias) Unclear risk All outcomes

Of the 16 participants initially enrolled: 'Two patients (one in each group) were diagnosed with cancer during the study and were not included in the analysis', i.e. $12.5 \%$ attrition, and no intention-to-treat analysis

Selective reporting (reporting bias) Unclear risk

Multiple outcome measures were used, with no primary outcome nominated, raising the risk of positive differences between the groups appearing by chance

\begin{tabular}{l|l}
\hline Other bias & Unclear risk
\end{tabular}

Multiple outcome measures were used, with no primary outcome nominated, raising the risk of positive differences between the groups appearing by chance 
Participants were initially randomly assigned to one of three group, but also matched by attending physiotherapist, nature of injury, rehabilitation stage, sport, level of participation, and gender. If a new study participant was matched to a participant already assigned to one of the three groups, that participants was allocation to one of the other two groups. When a third match was obtained, that person was assigned to the third group

Setting: Sports injury clinics in Wales, UK

Funding: Not reported

Recruitment (patients): Recruited from attendance at two sports injury clinics

Recruitment (healthcare providers): Not reported

Inclusion criteria (patients): People who had sustained a sports injury precluding participation in normal training and competition for a minimum of five weeks

Exclusion criteria (patients): Participants who could not be matched to two other participants in the study were omitted

Consideration of people with comorbidities: Not reported cruciate ligament injuries in the knee; $15.4 \%$ shoulder injuries; $7.7 \%$ lower leg fractures) Treatment currently receiving: Physiotherapy for sports injuries

Description of healthcare providers: Not reported

Study aim: To examine the effects of a five-week goal setting intervention (using proximal performance and process goals) on rehabilitation adherence, perceptions of self-efficacy and treatment efficacy, and on psychological responses to injury (e.g. self-confidence, loss of motivation and apathy)

Intervention: ( $\mathrm{n}=13$ ) Goal setting. Participants in this group met a sports psychologist every 7-10 days for five weeks (total number of sessions: four or five; duration: 60-105 minutes each session). During these sessions, a goal setting intervention was provided. Goals included proximal process (e.g. 'achieving a specific range of muscular tension in muscle groups targeted in rehabilitation' p. 314) and performance goals (e.g. 'completing a specified number of rehabilitation exercises or activity sessions). Goals were negotiated between the psychologist and participant; recorded on a goal-setting form designed for the study. The treating physiotherapist was not involved in the goal setting and goal monitoring process. Typically 2-5 goals were set. At following meetings, goal achievement was reviewed and recorded, and goals revised. Participants also completed a daily rehabilitation diary - recording personal thoughts on progress, emotional state etc Intervention (SSC): $(\mathrm{n}=13)$ Social support control (SSC). These participants met a sports psychologist every 7-10 days for five weeks (total number of sessions: four or five; duration: 40-60 minutes each session). The sport psychologist only provided social support, emotional support, listening support, shared social reality, task appreciation 'consistent with the type of support provided in the goal setting group' (p.314). Participants also completed a daily diary, recording same information as for the goal setting group

Control: $(\mathrm{n}=13)$ Received 5-10 min telephone call from sports psychologist every 10 days to encourage adherence to the study. Did not complete a daily diary, but did complete a training log 
Delivery: Goal setting and treatment occurred over a five-week period

Fidelity: Not reported

Consumer involvement outside of the intervention: Not reported

Outcomes

Goal setting characteristics
Timing of outcomes: Data collected at the end of the five weeks of intervention Self-reported rehabilitation adherence - daily recording of therapeutic exercises in a daily diary (for the goal setting and SSC groups) or an exercise log (control group) . Adherence was calculated from frequency of performing exercises prescribed by the treating physiotherapist

Overall physiotherapist estimate of patient adherence expressed as a single percentage value

Self-efficacy and treatment efficacy subscales of the Sports Injury Rehabilitation Beliefs Survey

'Dispirited' and 'reorganization' subscales of the Psychological Responses to Sports Injury Inventory

Comparison of interest: Collaborative goal setting plus strategies to enhance goal pursuit versus an attention control versus no additional clinical input

Patient involvement in goal setting: Goals collaboratively set by the patient and healthcare professional

Family involvement in goal setting: None reported

Name of goal setting approach: No specifically named approach used

Development of a plan for goal pursuit: Not specifically part of the goal setting approach used

Written copy of goals provided to patients: All goals recorded on a goal setting form designed for the study, but it is not clear whether this form was held by the patient or sports psychologist involved

Individual versus group-based goal discussion: Individual

Level of goal difficulty: Not reported

Goal areas of focus: Impairments of body structure and function or activity limitations

Evaluation of patient goal commitment: Not reported

Goal reminders used: Goals reviewed at weekly meetings

Monitoring of progress towards goals: Goal progress discussed at weekly meetings

\section{Risk of bias}

\section{Bias}

Random sequence generation (selection High risk bias)

\section{Authors' judgement}

\section{Support for judgement}

'Participants were randomly assigned to one of three groups... [but] were matched across groups according to the attending physiotherapists, nature of the injury, rehabilitation stage, sport, level of participation, and gender. When a match was obtained for a participant already assigned to a group, the new participant was randomly assigned to one of the other two groups. 
Evans 2002 (Continued)

When two matches had already been obtained and assigned to a group, and a third match was identified, that participant was assigned to the remaining group. Participants who could not be matched across the three groups according to the criteria were subsequently omitted from the study $(\mathrm{N}=$ 38)’ (p.312)

Allocation concealment (selection bias) High risk

Allocation not concealed due to matching of participants across study group during the recruitment process

Blinding of participants and personnel Unclear risk (performance bias)

All outcomes

The participants were told that the purpose of the study was to examine the psychological responses and rehabilitation adherence, but were blinded to the experimental manipulation of goal setting variables. The physiotherapists providing the physical intervention were blinded to the group that patients were assigned. The sports psychologist providing the goal setting could not be blinded to group allocation because their active involvement in the delivery of the intervention was required

Blinding of outcome assessment (detection Unclear risk bias)

Most outcomes were based on participant self-report, with participants knowing what intervention they received but not knowing about the experimental manipulation of goal setting variables. Overall physiotherapist estimate of patient adherence scores were based on the reporting of therapists blinded to group allocation

Incomplete outcome data (attrition bias) Low risk

No reported attrition. All participants acAll outcomes counted for in the data analysis

Selective reporting (reporting bias) Unclear risk

Unable to find a protocol for this study published prior to it being conducted, so unable to compare the outcomes reported with those planned to be measured at the outset

Other bias

Low risk

No evidence of other sources of bias 


$\begin{array}{ll}\text { Methods } & \text { RCT } \\ \text { Setting: Private community mental health clinic in New York, USA } \\ \text { Funding: Not reported. Completed as a PhD thesis } \\ \text { Recruitment (patients): Clients from a private mental health clinic. 'A convenience sam- } \\ \text { ple of } 30 \text { subjects was drawn from new and active clients who were receiving individual } \\ \text { counselling from the clinic [Community Mental Health Nurse] CMHN research and } \\ \text { the clinical CMHN research assistant who conducted this study.' (p.51) } \\ \text { Recruitment (healthcare providers): Not reported } \\ \text { Inclusion criteria (patients): Having mental health condition (as classified by the DSM- } \\ \text { III-R) and be attending a mental health clinic for therapy services } \\ \text { Exclusion criteria (patients): No further criteria reported } \\ \text { Consideration of people with comorbidities: Not reported }\end{array}$
with exhibit reduced stress in comparison to community mental health clients who do not participate in mutual goals setting as part of their counselling service

Intervention: ( $n=15)$ Mutual goal setting plus counselling. Attended four 1-hour counselling session with a community mental health nurse, during which mutual goals were established. Goal focused on addressing identified stressors, and involved the participant and nurse agreeing on interventions to achieve the goal. This was followed by a period of regular counselling, involving either 1-hour weekly for four weeks or bi-weekly sessions for two weeks

Control: ( $\mathrm{n}=15)$ Attended four 1-hour counselling sessions with a mental health nurse on a weekly or bi-weekly basis, without discussion of goals

Delivery: Goal setting and counselling were delivered over a 2-4 week period

Fidelity: Not reported

Consumer involvement outside of the intervention: Not reported

Outcomes

Timing of outcomes: On completion of the fourth weekly or bi-weekly session (i.e. after 2-4 weeks)

Derogatis Stress Profile Instrument (DSP)

Goal setting characteristics

Comparison of interest: Collaborative goal setting versus attention control with no goal setting

Patient involvement in goal setting: Goals collaboratively set by the patient and healthcare professional

Family involvement in goal setting: None reported

Name of goal setting approach: Based on Imogene King's theory of goal attainment (King 1981)

Development of a plan for goal pursuit: A plan for goal pursuit was collaboratively set by the patient and healthcare professional 
Fredenburgh 1993 (Continued)

Written copy of goals provided to patients: Not reported

Individual versus group-based goal discussion: Individual

Level of goal difficulty: Not reported

Goal areas of focus: Not reported

Evaluation of patient goal commitment: Not reported

Goal reminders used: None

Monitoring of progress towards goals: None

Notes

Power calculation: Not reported

\section{Risk of bias}

\begin{tabular}{l|l} 
Bias & Authors' \\
\hline $\begin{array}{l}\text { Random sequence generation (selection } \\
\text { bias) }\end{array}$ & Low risk
\end{tabular}

bias)

'A lottery method was used to randomly assign the 30 subjects into the experimental and control groups... Each participant in the sample was assigned a numerical identification number. Corresponding sequential numbers were marked on slips of paper and placed into a bag. The slips were thoroughly mixed in the bag. A research assistant drew a numbered slip, recorded the number on a sheet of paper, and placed the slip of paper back into the bag. By returning the slips back into the bag, each subject had an equal chance of being selected. During the drawing, if the same number was drawn, the second drawing was ignored, and the number was placed back into the bag. The first 15 numbers drawn were assigned to the experimental group, and the remaining 15 to the control group' (p. 5253)

\begin{tabular}{l|l|l} 
Allocation concealment (selection bias) & Low risk & See above \\
\hline $\begin{array}{l}\text { Blinding of participants and personnel } \\
\text { (performance bias) } \\
\text { All outcomes }\end{array}$ & High risk & $\begin{array}{l}\text { The intervention required active involve- } \\
\text { ment of the patient and healthcare profes- } \\
\text { sionals, so blinding not possible }\end{array}$ \\
\hline $\begin{array}{l}\text { Blinding of outcome assessment (detection } \\
\text { bias) } \\
\text { All outcomes }\end{array}$ & High risk & $\begin{array}{l}\text { Data collection was based on self-report by } \\
\text { participants who, by necessity of the inter- } \\
\text { vention, knew which intervention they re- } \\
\text { ceived }\end{array}$ \\
\hline
\end{tabular}

Incomplete outcome data (attrition bias) Low risk All outcomes
No reported attrition. All participants accounted for in the data analysis 
Fredenburgh 1993 (Continued)

\begin{tabular}{|c|c|c|}
\hline Selective reporting (reporting bias) & Unclear risk & $\begin{array}{l}\text { Unable to find a protocol for this study } \\
\text { published prior to it being conducted, so } \\
\text { unable to compare the outcomes reported } \\
\text { with those planned to be measured at the } \\
\text { outset }\end{array}$ \\
\hline
\end{tabular}

Other bias

Unclear risk

The potential for cross-group contamination in this study was unclear. There was one nurse providing both the intervention and control therapies. The possible interactions between participants was unclear

Gagné 2003

Methods

Quasi-RCT

Setting: A rehabilitation hospital in Texas, USA

Funding: Not reported

Recruitment (patients): Recruited from hospital admissions

Recruitment (healthcare providers): Not reported

Inclusion criteria (patients): length of stay in hospital anticipated to be more than two weeks, score on admission of 25 or less in the six area of self-care on the Functional Independence Measure (indicating need for assistance to complete component areas of those tasks)

Exclusion criteria (patients): Mini Mental State Exam score of less than 25

Consideration of people with comorbidities: Not reported

Participants

Patients: 31 participants (12.9\% male). Mean age and SD not reported. Range 56 to 93 years. Ethnicity not reported

Principle health problems: Participants 'were being treated for neurological, cardiopulmonary, and orthopaedic deficits, back injury, and debilitation' (p. 216)

Treatment currently receiving: Inpatient physiotherapy and occupational therapy. Other type of medical, nursing or therapy care may be also been provided but not reported Description of healthcare providers: Registered occupational therapists and occupational therapy assistants Demographic characteristics of providers not reported

Interventions

Study aim: Two research questions were explored: '1) Will therapy that includes daily recording and communication of goals with a therapist improve self-care outcomes to a greater extent than occupational therapy that does not focus on goals in this manner, and 2) Will all self-care areas be affected in a similar way by goal-focused therapy?' (p. 216)

Intervention: ( $\mathrm{n}=15$ ) Occupational therapy plus enhanced goal setting. Participants were given a goal notebook. They engaged collaboratively with their therapists in daily discussions and written documentation of goals. Each goal contained a subject (the participant), and observable action verb, a functional performance, a condition under which the performance was to be met, and the criteria required to complete the performance measure (such as time, accuracy, distance, speed, or quality of movement) Control: ( $n=16)$ Occupational therapy with non-enhanced goal setting. Between 90-135 minutes of occupational therapy per day, including individual and group therapy session 
of up to 45 minutes per session. Participants set goals with their therapists initially, but during daily therapy goals were not emphasised or specifically mentioned or reviewed. Instead, participants were urged to do their best with self-care tasks. Participants were not given goal notebooks. All communication about initial goals was verbal

Delivery: Occupational therapy and goal setting was delivered over a two-week period Fidelity: The principal investigator continuously tracked goal statements to ensure therapists were following protocols. Treatment fidelity was not reported however Consumer involvement outside of the intervention: Not reported

Outcomes

Timing of outcomes: Recorded duration admission, then again after 2 weeks of occupational therapy

Functional Independence Measure (FIM) using just the six self-care sub-domains for upper body dressing, lower body dressing, bathing, eating, grooming, and toileting

Goal setting characteristics

Comparison of interest: Collaborative goal setting plus strategies to enhance goal pursuit versus usual care with no standardised or required approach to goal setting

Patient involvement in goal setting: Goals collaboratively set by the patient and healthcare professional

Family involvement in goal setting: None reported

Name of goal setting approach: No specifically named approach used

Development of a plan for goal pursuit: Not reported

Written copy of goals provided to patients: Patient were provided with a goal notebook in which their goals for therapy were documented

Individual versus group-based goal discussion: Individual

Level of goal difficulty: Not reported

Goal areas of focus: Functional performance

Evaluation of patient goal commitment: Not reported

Goal reminders used: The goal notebooks were used to remind patients and therapists about the goals

Monitoring of progress towards goals: The goal notebooks were used to enhanced discussions between patients and therapists regarding goal progress, but it is not clear whether this included specifically measuring progress towards goals during the course of therapy

\section{Risk of bias}

\begin{tabular}{|c|c|c|}
\hline Bias & Authors' judgement & Support for judgement \\
\hline $\begin{array}{l}\text { Random sequence generation (selection } \\
\text { bias) }\end{array}$ & High risk & $\begin{array}{l}\text { 'After meeting inclusion criteria, assign- } \\
\text { ment to groups was done by convenience, } \\
\text { based on room-assignment on the rehabil- } \\
\text { itation unit' (p.216) }\end{array}$ \\
\hline Allocation concealment (selection bias) & High risk & See above \\
\hline
\end{tabular}


Gagné 2003 (Continued)

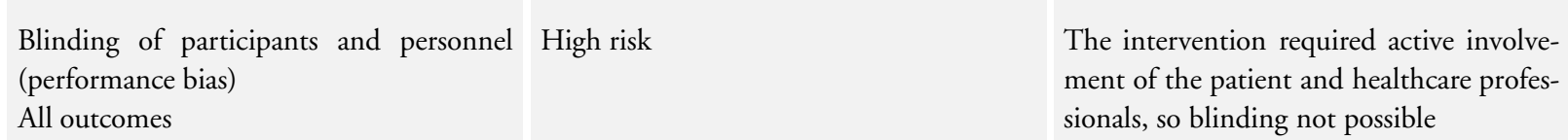

Blinding of outcome assessment (detection Low risk

bias)

All outcomes

'Therapists collecting data were unaware of expectations and projected outcomes as well as the treatment assignment of the participants' (p.217)

Incomplete outcome data (attrition bias) Low risk

No reported attrition. All participants acAll outcomes counted for in the data analysis

Selective reporting (reporting bias) Unclear risk

Unable to find a protocol for this study published prior to it being conducted, so unable to compare the outcomes reported with those planned to be measured at the outset

Other bias

Unclear risk

Unclear potential for cross-group contamination. Unclear whether there was any variability in the therapy received by two study groups, or the extent of this if treatment variability did exist. "Attention was not given to specific treatment protocols; therefore, treatment activity may have varied significantly between therapists and as a result between groups" (p.217-218)

Hart 1978

Methods

RCT

Setting: A community mental health centre in Utah, USA

Funding: Not reported

Recruitment (patients): Not reported

Recruitment (healthcare providers): Not reported

Inclusion criteria (patients): Community-based mental health consumers for whom 1) short-term (three months) individual psychotherapy was deemed clinically appropriate and 2) meaningful and responsible participation in a therapy program was deemed possible

Exclusion criteria (patients): No further criteria reported

Consideration of people with comorbidities: Not reported

Participants

Patients: 32 participants. No descriptive data about the patient population was reported Principle health problems: Mental health disorders

Treatment currently receiving: Individual psychotherapy

Description of healthcare providers: Eight psychotherapists. Demographic characteristics of providers not reported

Goal setting and strategies to enhance goal pursuit for adults with acquired disability participating in rehabilitation (Review) 
Hart 1978 (Continued)

Interventions
Study aim: In the context of psychotherapy for community-based people with mental health condition, '... to evaluate the therapeutic effectiveness of setting goals in behavioral terms while monitoring the subject's progress in attaining these goals' (p.1242). In the context of this study, 'therapeutic effectiveness' was evaluated by goal attainment

Before randomisation: Participants received two sessions of individual therapy involving history-taking. At a third therapy session, the participants worked with a healthcare provider trained in use of GAS to set GAS goals to be achieved by the eighth session. This healthcare provider also interviewed other people 'significant to the patient's problem area' (p. 1243) before setting goals with the participant. Demographic characteristics of the significant others were not reported

Intervention ( $\mathrm{n}=$ not reported): Individual psychotherapy plus goal discussion. Participants received five further weekly psychotherapy sessions (sessions four through to eight) which included discussion of goal progress using a structure feedback technique (the Behavioral Monitoring Progress Report)

Control ( $\mathrm{n}=$ not reported): Individual psychotherapy without goal discussion: Participants received five further weekly psychotherapy sessions (sessions four through to eight) without any structured discussion of goal progress

Delivery: Therapy and goal setting delivered over eight weeks

Fidelity: Not reported

Consumer involvement outside of the intervention: Not reported

Outcomes

Timing of outcomes: Reported at a three-month period, although is not clear whether this was three months from enrolment in the study or from the end of therapy or from some other time point

Change in GAS scores from session three, calculated as T-score. Two GAS scores were provided: one scored by the patients and one by their 'collateral' (a significant person in their life)

Goal setting characteristics
Comparison of interest: Collaborative goal setting plus strategies to enhance goal pursuit versus usual care with no goal setting

Patient involvement in goal setting: Goals collaboratively set by the patient and healthcare professional

Family involvement in goal setting: A 'collateral' person was involved in setting goals and evaluating their achievement, but who this person was, and their relationship to the participants, was not reported

Name of goal setting approach: GAS

Development of a plan for goal pursuit: Behavioural assignments were set each week, which included a weekly goal and method of attainment

Written copy of goals provided to patients: Not reported

Individual versus group-based goal discussion: Individual

Level of goal difficulty: Not reported

Goal areas of focus: Not reported

Evaluation of patient goal commitment: Not reported

Goal reminders used: Weekly discussion of the patients' goals served a goal reminder

Monitoring of progress towards goals: A Behavioural Monitoring Progress Record was completed and discussed weekly by the patient and therapist 
Hart 1978 (Continued)

\section{Risk of bias}

\begin{tabular}{|c|c|c|}
\hline Bias & Authors' judgement & Support for judgement \\
\hline $\begin{array}{l}\text { Random sequence generation (selection } \\
\text { bias) }\end{array}$ & Unclear risk & $\begin{array}{l}\text { 'The patients... were randomly assigned to } \\
\text { the two treatment groups...' (p.1244). In- } \\
\text { sufficient information provided to deter- } \\
\text { mine whether adequate sequence genera- } \\
\text { tion }\end{array}$ \\
\hline Allocation concealment (selection bias) & Unclear risk & No information provided \\
\hline $\begin{array}{l}\text { Blinding of participants and personnel } \\
\text { (performance bias) } \\
\text { All outcomes }\end{array}$ & High risk & $\begin{array}{l}\text { The intervention required active involve- } \\
\text { ment of the patient and healthcare profes- } \\
\text { sionals, so blinding not possible }\end{array}$ \\
\hline $\begin{array}{l}\text { Blinding of outcome assessment (detection } \\
\text { bias) } \\
\text { All outcomes }\end{array}$ & High risk & $\begin{array}{l}\text { The GAS scales used for outcome assess- } \\
\text { ment were created and individualised be- } \\
\text { fore patients were randomly allocated to } \\
\text { the two study groups. The GAS outcome } \\
\text { data were collected 'by one of four mas- } \\
\text { ter's level psychiatric nurses'. It is not re- } \\
\text { ported whether these nurses were blinded } \\
\text { to group allocation. The GAS data was } \\
\text { also based on self-report by patients (not } \\
\text { blinded) and their significant others (not } \\
\text { reported whether blinded or not) }\end{array}$ \\
\hline $\begin{array}{l}\text { Incomplete outcome data (attrition bias) } \\
\text { All outcomes }\end{array}$ & Unclear risk & No information provided \\
\hline Selective reporting (reporting bias) & Unclear risk & $\begin{array}{l}\text { Unable to find a protocol for this study } \\
\text { published prior to it being conducted, so } \\
\text { unable to compare the outcomes reported } \\
\text { with those planned to be measured at the } \\
\text { outset }\end{array}$ \\
\hline Other bias & Unclear risk & $\begin{array}{l}\text { Potential for cross-group contamination } \\
\text { unclear }\end{array}$ \\
\hline
\end{tabular}




\section{Methods}

RCT

Setting: Post-acute rehabilitation service in Pennsylvania, USA

Funding: National Institute on Disability and Rehabilitation Research

Recruitment (patients): Participant recruited from a community re-entry programme and a 'clubhouse' day programme

Recruitment (healthcare providers): Not reported

Inclusion criteria (patients): documented history of memory impairments observed at a functional level; confirmation from the case manager or other involved healthcare providers of significant memory problems; involved in a 'comprehensive' treatment programme for two to five days per week (i.e. needed to be working on more than two goal areas in rehabilitation)

Exclusion criteria (patients): severe receptive or expressive communication problems Consideration of people with comorbidities: Not reported

\section{Participants}

Patients: 10 participants (80\% male). Mean age 31.5 (SD 7.1). Range 19 to 45 years. Ethnicity not reported

Principle health problems: Traumatic brain injury with significant ongoing memory problems. Three months to 18 years post-injury

Description of healthcare providers: Not reported
Interventions
Study aim: To determine whether use of an electronic device (a portable voice organizer) could help people with traumatic brain injury recall their current treatment goals Intervention ( $\mathrm{n}=30$ goals): Portable Voice Organiser reminders of rehabilitation goals. The participants' case manager selected six current therapy goals. Goal included longerterm goal, i.e. 'broad statements expressing the 'big picture' of treatment' (p.560), as well as 'more focused or specific goals' (p.560). Each goal was expressed as a single sentence that the participant could understand. Training was provided to patients on use of the Portable Voice Organiser. Three goals were then selected at random to be verbally recorded on the Portable Voice Organiser, and played back to the participants three consistent times per day for seven days. An alarm system was used to notify when the participant should listen to the Portable Voice Organiser

Control ( $\mathrm{n}=30$ goals): As above, but no recorded messages about these goals were stored on the Portable Voice Organiser

Delivery: Training in use of the Portable Voice Organiser occurred over three sessions or less (as needed) Delivery of the Portable Voice Organiser intervention occurred over a seven-day period

Fidelity: Before the trial began, participants were providing individual training in use of the device. Success of training was tested. Some researcher interaction around the device was scripted. Researchers also monitored (from a distance) the participants use of the Portable Voice Organiser and kept a check on whether or not there were any problems with the equipment over the seven day trail period. One of ten participants had to restart the study period because of difficulty with the equipment

Consumer involvement outside of the intervention: Impetus for the trial came from a series of focus groups with healthcare providers involve with traumatic brain injury rehabilitation, but involvement of consumers in the development of the therapy or study was not reported

Outcomes

Timing of outcomes: Seven days after goals first stored on the Portable Voice Organiser Recall of goals score on a four-point scale. Two types of recall examined: 1 . free recall (based on open-ended question re. the participant's goals) and cued recall (seeing if the 
Hart 2002 (Continued)

participant can remember their goals based on their negotiated 'cue words')

Goal setting characteristics

Comparison of interest: Enhanced approach to improving goal recall versus usual care Patient involvement in goal setting: Not reported

Family involvement in goal setting: None reported

Name of goal setting approach: No specifically named approach used

Development of a plan for goal pursuit: Not the subject of the research

Written copy of goals provided to patients: Usual practice around documentation of goals not reported

Individual versus group-based goal discussion: Individual

Level of goal difficulty: Not reported

Goal areas of focus: Not reported

Evaluation of patient goal commitment: Not reported

Goal reminders used: The study involved comparison of Portable Voice Organizer reminders of goals versus no additional goal reminders

Monitoring of progress towards goals: Not the subject of the research

Notes

Power calculation: Not reported

Risk of bias

\begin{tabular}{lll}
\hline Bias & Authors' judgement & Support for judgement
\end{tabular}

Random sequence generation (selection Low risk bias)

'...the goals were randomly assigned to be recorded (three) or unrecorded (three) by blind selection of identical slips of paper' (p.561)

\begin{tabular}{l|l|l}
\hline Allocation concealment (selection bias) & Low risk & See above \\
\hline $\begin{array}{l}\text { Blinding of participants and personnel } \\
\text { (performance bias) }\end{array}$ & High risk & $\begin{array}{l}\text { The intervention was automated by a } \\
\text { Pll outcomes }\end{array}$ \\
\end{tabular}

Blinding of outcome assessment (detection Unclear risk bias)

All outcomes
'... each participant's recall for the six goals was tested by a project staff member who was blind both to the therapy goals relevant to that patient and to the specific goals that had been recorded' (p.562). However, the participants were not blinded to group allocation, and the outcome measures were based on their self-report

No evidence of attrition

Incomplete outcome data (attrition bias) Low risk All outcomes 
Hart 2002 (Continued)

\begin{tabular}{l|l|l}
\hline Selective reporting (reporting bias) & Unclear risk & $\begin{array}{l}\text { Unable to find a protocol for this study } \\
\text { published prior to it being conducted, so } \\
\text { unable to compare the outcomes reported } \\
\text { with those planned to be measured at the } \\
\text { outset }\end{array}$ \\
\hline Other bias & Low risk & No evidence of other sources of bias \\
\hline
\end{tabular}

Harwood 2012

Methods
RCT

Setting: Community, New Zealand

Funding: Health Research Council of New Zealand and the B Basham Medical Charitable Trust

Recruitment (patients): Recruited from the community, but the majority were first identified during a hospital stay for acute stroke, with a small number of others recruited via community-based organisations such as the New Zealand Stroke Foundation

Recruitment (healthcare providers): Not reported

Inclusion criteria (patients): Inclusion criteria: over 15 years of age; six to 12 weeks after stroke; living outside institutional care; self-identifying as Maori or Pacific ethnicity

Exclusion criteria (patients): No further criteria reported

Consideration of people with comorbidities: Concurrent diagnoses of diabetes (41\%) and high body mass index (mean 32.1; SD 12.7) were reported
Patients: 172 participants (gender not reported). Mean age 61 (SD 14). 54.7\% Maori; 45.3\% Pacific peoples (comprised of 26.7\% Samoan; 7.6\% Cook Island Maori; $7.0 \%$ Tongan; $1.1 \%$ Niuean; $0.6 \%$ Tokelauan; $0.6 \%$ Tahiti/Pitcairn; 0.6\% Fijian; $1.1 \%$ Pacific not otherwise stated)

Principle health problems: Stroke, with $26.2 \%$ having had a previous stroke; activity limitations mild-to-moderate (Barthel Index mean scores 16.7, SD 4.7); 38.6\% dependent on others (modified Rankin Score> 2)

Treatment currently receiving: Any use of rehabilitation service in addition to those provided as part of the study was one of the outcome measures, but the details of these additional services was not reported

Description of healthcare providers: All interventions were provided by research assistants from the same ethnic group as the participant. A minimum of five day's training was provided to these research assistants prior to starting the study, and throughout the duration of the study

Interventions

Study aim: To test the effect of an inspirational DVD versus a 'Take Charge' intervention versus both the DVD and 'Take Charge' intervention versus a usual care control group in terms of improvements in function and quality of life one year after stroke Intervention (Take Charge): ( $\mathrm{n}=46)$ A 80-minute structured, individualised assessment of risk factors for dependence and activities of daily living, delivered by a person of the same ethnicity as the person with stroke, leading the patient and their family to identify areas for personal progress and personal goal setting. A structured format used with the following headings: physical, communication, emotion/mood, information needs, financial, whanau (extended family), secondary prevention. A booklet was used, with 
Harwood 2012 (Continued)

each heading on a separate page. On these were recorded: the date, goals in own words, rehabilitation assessment, specific objectives, time frames, and how to achieve these. Goals were viewed as an ongoing process where new goals could be set. No actual therapy was provided

Intervention (Inspiration DVD): $(\mathrm{n}=48)$ An 80-minute DVD about stroke and stroke recovery using inspirational stories of four Maori and Pacific people and their families, told by them, containing the following dominant messages: potential for good outcomes, overcoming adversity, personal and family roles in contributing to recovery, encouraging meaningful activity, participation after stroke, accessing resources after stroke. The DVD was left with the participants and they were encouraged to view it as often as they wished Intervention (Take Charge + Inspirational DVD): $(\mathrm{n}=39)$ A combination of both the 'Take Charge' session and the inspirational DVD intervention

Control: $(\mathrm{n}=39)$ Received neither the 'Take Charge' intervention nor the inspirational DVD, but did get written material about stroke for people and their families, delivered by a research assistant of the same ethnicity as the person with stroke, during a 30-minute visit

Delivery: All interventions delivered over a single community-based visit

Fidelity: Evaluation of fidelity to the intervention protocols was not reported, but training was provide to the people providing the interventions and structured around specific resources, minimising potential for cross-group contamination

Consumer involvement outside of the intervention: The interventions were development from qualitative research on the experience of Maori and Pacific peoples with stroke

Outcomes

Timing of outcomes: 12 months after randomisation

Short Form 36 - Physical Component Summary Score

Short Form 36 - Mental Component Summary Score

Barthel Index

Frenchay Activities Index

Systolic blood pressure

Dependency (treated as a dichotomous variable, with a modified Rankin score of over 2 indicating 'dependency')

Use of rehabilitation service (treated as a dichotomous variable, 'any' versus 'no' face-toface input from a rehabilitation healthcare provider)

Mortality

Goal setting characteristics

Comparison of interest: Training in self-directed goal setting plus strategies for goal pursuit versus an inspirational DVD versus training in self-directed goal setting plus strategies for goal pursuit and an inspirational DVD versus no additional input

Patient involvement in goal setting: Patient (with family) were trained in setting their own goals (i.e. self-directed rehabilitation)

Family involvement in goal setting: Family directly involved in the goal setting process Name of goal setting approach: No specifically named approach used

Development of a plan for goal pursuit: A booklet was used to help patients collect their own assessment information, document their own goals, set objectives, and plan how to achieve them

Written copy of goals provided to patients: Patients document their own goals Individual versus group-based goal discussion: Family-based goal discussion Level of goal difficulty: Not specified. Left up to the patient and their family to decide Goal areas of focus: Structured around topic in the booklets (e.g. functional abilities, 
Harwood 2012 (Continued)

emotional needs, information needs, financial needs, family needs, secondary prevention) but ultimately left up to the patient and their family to decide

Evaluation of patient goal commitment: Not reported

Goal reminders used: None

Monitoring of progress towards goals: None. Left up to the patient and their family to self-monitor

Notes

Power calculation: The researchers aimed to enrol 240 people so that the study would have $80 \%$ power to detect a difference of four units in the Physical Component Summary Score on the SF-36 with alpha of 5\% and assuming a dropout rate of $15 \%$. This target was not reached, with 172 people being eventually randomised to the four treatment groups

\section{Risk of bias}

\begin{tabular}{lll}
\hline Bias & Authors' judgement & Support for judgement \\
\hline $\begin{array}{l}\text { Random sequence generation (selection } \\
\text { bias) }\end{array}$ & Low risk & $\begin{array}{l}\text { 'Randomization was achieved using a ran- } \\
\text { dom numbers table with stratification by } \\
\text { ethnic group (Maori or Pacific)...' (p.496) }\end{array}$ \\
\hline Allocation concealment (selection bias) & Low risk & 'Randomization was achieved using... \\
opaque sealed envelopes to conceal alloca- \\
tion' (p. 496)
\end{tabular}

Blinding of participants and personnel High risk (performance bias)

All outcomes

Blinding of outcome assessment (detection Unclear risk bias)

All outcomes

Incomplete outcome data (attrition bias) High risk All outcomes
The intervention required active involvement of the patient and research assistant, so blinding not possible

'Outcome assessments were performed by research assistants masked to treatment allocation' (p.496). However, some outcomes, including the primary outcome, were based on self-reported by participants who were not blinded to group allocation

'The analysis was by intention to treat. No attempt was made to impute values for those with missing data as it was strongly suspected that the reason for missing data was related to the values of the outcome variables (i.e. the missing data were not missing at random).' However, 33/172 (19. $2 \%$ ) were lost to follow-up, so only $80.8 \%$ of the initially enrolled participants were included in data set for analysis 
Harwood 2012 (Continued)

\begin{tabular}{l|l|l} 
Selective reporting (reporting bias) & Low risk & $\begin{array}{l}\text { No evidence of selective reporting. Re- } \\
\text { ported outcomes match those proposed in } \\
\text { a protocol published in the Australian New } \\
\text { Zealand Clinical Trials Registry prior to } \\
\text { undertaking the study }\end{array}$ \\
\hline Other bias & Low risk & No evidence of other sources of bias \\
\hline
\end{tabular}

Holliday 2007

Methods

Quasi-RCT. A repetitive block design was used with each block lasting three months, and with all patients admitted to a rehabilitation unit recruited to the study over an 18month period

Setting: An 18-bed hospital-based neurorehabilitation unit in London, UK

Funding: UCLH Clinical Research and Development Committee

Recruitment (patients): Recruited from hospital inpatients over an 18-month period

Recruitment (healthcare providers): Staff members within the neurorehabilitation unit

Inclusion criteria (patients): All patients admitted to the rehabilitation unit over an 18

month period regardless of diagnosis

Exclusion criteria (patients): inability to speak English; severe dysphasia

Consideration of people with comorbidities: Not reported

Participants

Patients: 201 participants. Demographic data not reported, but the authors state that there was no significant difference between the two groups in terms of gender, ethnicity, functional status, or case mix

Principle health problems: Neurological disorders

Treatment currently receiving: Inpatient multidisciplinary rehabilitation

Description of healthcare providers: An established multidisciplinary neurorehabilitation team

Interventions

Study aim: To examine the impact of a protocol to increase patient participation in goal setting on patient autonomy and level of functional abilities

Intervention: $(n=101)$ Increased participation in goal setting. A goal setting workbook was used by patients in this group, plus the participants had greater involvement in goal planning meetings. 'The workbook was in three sections. Participants worked through these with support from family and friends, then, if needed, from their keyworker. The first section asked patients to prioritise activity and participation domains; the second section asked to identify specific tasks within those domains that they wished to work on; the final section involved determining what individuals wanted to achieve within the time frame of the rehabilitation admission. Goal setting meetings provided a formal opportunity for therapists to discuss with patients both the projected outcome and the reasons for this. Patient were then encouraged to set realistic goals' (p.577)

Control: $(n=100)$ Usual practice. Healthcare providers followed a care pathway which incorporated setting goals at the level of body function, activity, and participation roles, using up to four of 26 goal components grouped under five areas: 1) health maintenance, 2) cognitive functioning, 3) personal activities of daily living, 4) participation, and 5) communication. No goal setting workbook was used and patient were not present during the team's goal setting meetings 
Holliday 2007 (Continued)

Delivery: Each approach goal setting was delivered to all patients admitted to the neurorehabilitation unit during a three month period, with only one approach being implemented at a time during alternate three month periods over an 18-month timeframe Fidelity: Evaluation of fidelity not reported

Consumer involvement outside of the intervention: No

Outcomes

Timing of outcomes: Not reported. Presumed to be at the end of inpatient rehabilitation Functional Independence Measure

London Handicap Scale

General Health Questionnaire

Self-reported involvement in goal setting (based on a four-point patient autonomy scale derived from the Patient Participation Scale)

Self-reported goal relevance using a $10 \mathrm{~cm}$ visual analogue scale and five-point categorical scale

Patient overall satisfaction using a $10 \mathrm{~cm}$ visual analogue scale

Goal setting characteristics

Comparison of interest: Collaborative goal setting plus strategies to enhance goal pursuit versus usual care with less patient involvement in goal setting

Patient involvement in goal setting: Goals collaboratively set by the patient and healthcare professional

Family involvement in goal setting: Support from family encouraged but not required

Name of goal setting approach: No specifically named approach used

Development of a plan for goal pursuit: Goals to be achieved by discharge were broken down into short terms goals to be achieved as step toward the discharge goal. Short-term goals were revised as the admission progressed

Written copy of goals provided to patients: Not reported

Individual versus group-based goal discussion: Individual

Level of goal difficulty: Setting 'realistic' goals was emphasised

Goal areas of focus: Activity limitations and participation restrictions

Evaluation of patient goal commitment: Not reported

Goal reminders used: Not reported

Monitoring of progress towards goals: Not reported

Notes

Power calculation: 'We calculated that 100 patients in each arm would enable us to detect a significant difference in functional outcome at the p,0.05 level' (p.577). However, later the authors reported: 'We did not anticipate any impact on function, as measured using disability scales, as the intervention time was similar in both groups' (p.578), making the basis for the sample size unclear

Risk of bias

\begin{tabular}{|c|}
\hline Bias \\
\hline
\end{tabular}

Random sequence generation (selection High risk

Allocation of study groups was by date of bias) admission

Allocation concealment (selection bias) High risk

See above

Goal setting and strategies to enhance goal pursuit for adults with acquired disability participating in rehabilitation (Review) 
Holliday 2007 (Continued)

Blinding of participants and personnel High risk (performance bias)

All outcomes
The intervention required active involvement of the patient and healthcare professionals, so blinding not possible

No blinding of outcome assessment bias)

All outcomes

Incomplete outcome data (attrition bias) Low risk All outcomes
No evidence of attrition, with all participants accounted for in the analysis

Unable to find a protocol for this study published prior to it being conducted, so unable to compare the outcomes reported with those planned to be measured at the outset
All staff in the rehabilitation unit were involved in treating participants in both groups of the study, and there were periods during the study when participants in both groups were present on the ward at the same time, therefore the risk of cross-group contamination was high (author communication)

Howell 1986

Methods

Participants
RCT

Setting: A district service for people with persistent and intransigent psychiatric disorders in London, UK

Funding: Not reported

Recruitment (patients): Randomly chosen from day patients and inpatients attending the district service

Recruitment (healthcare providers): Recruited from staff working in the service

Inclusion criteria (patients): No further criteria reported apart from being a person attending the district service

Exclusion criteria (patients): No further criteria reported

Consideration of people with comorbidities: Not reported

Patients: 27 participants enrolled. 24 participants completed the study ( $58.3 \%$ male). Mean age 44 (SD 14). Range 20 to 69 years. Ethnicity not reported

Principle health problems: Mental health disorders. 79.2\% with psychoses diagnosis; 20. $8 \%$ with other mental health conditions. Mean number of years since first diagnosis 15 . 71 (SD 6.96). Range 4 to 38 years

Treatment currently receiving: Not reported, other than being in a community facility for mental health conditions

Description of healthcare providers: Six occupational therapists, randomly assigned to 


\section{Howell 1986 (Continued)}

the treatment or the control group. Demographic characteristics of the occupational therapists not reported

Interventions
Study aim: To test the hypothesis that people with mental health conditions who receive weekly individualised goal setting and goal planning would achieve a higher level of goal attainment when compared to a control group who received an equivalent amount of time for positive social reinforcement alone

Intervention: ( $\mathrm{n}=$ not reported, number completing intervention $=11$ ) Occupational therapy directed toward achieving collaboratively set goals, based on use of GAS. Sessions involved goal identification, goal documentation, development of plans for goal achievement, and reviewing of goal progress

Control: ( $\mathrm{n}=$ not reported, number completing intervention $=13)$ Occupational therapy consisting of weekly clinical reviews with positive social reinforcement and no formal, structured goal setting

Delivery: Both intervention and control therapy was delivered during weekly 10 -minute occupational therapy sessions over a period of eight weeks

Fidelity: Not evaluated

Consumer involvement outside of the intervention: No
Outcomes

Goal setting characteristics
Timing of outcomes: After 8 weeks of intervention

Griffiths work performance scale

Shepherd Social Behaviour Rating Scale

GAS. 'Theoretical' GAS goals were set by the researcher and experimental group occupational therapists for participants, based on tape recordings of the clinical team's routine discussions. These goals were separate to the 'actual' GAS goals set during the intervention sessions with the patients. The GAS method was extended to a 7-point scale for the purposes of this study, but the details of what this involved are unclear. Goal attainment also dichotomised as 'high' if the GAS score was greater than or equal to 5 or 'low' is less than 5

Comparison of interest: Collaborative goal setting plus strategies to enhance goal pursuit versus attention control with no goal setting

Patient involvement in goal setting: Goals collaboratively set by the patient and healthcare professional

Family involvement in goal setting: None reported

Name of goal setting approach: GAS

Development of a plan for goal pursuit: Developed during weekly meetings between the patient and therapist

Written copy of goals provided to patients: Not reported

Individual versus group-based goal discussion: Individual

Level of goal difficulty: Not reported

Goal areas of focus: Not reported

Evaluation of patient goal commitment: Not reported

Goal reminders used: The intervention included weekly meeting where goals and subgoal plans were discussed

Monitoring of progress towards goals: Not reported 


\section{Howell 1986 (Continued)}

\section{Risk of bias}

\begin{tabular}{|c|c|c|}
\hline Bias & Authors' judgement & Support for judgement \\
\hline $\begin{array}{l}\text { Random sequence generation (selection } \\
\text { bias) }\end{array}$ & Unclear risk & $\begin{array}{l}\text { Participants '...were randomly assigned to } \\
\text { the treatment or control group' (p.265). In- } \\
\text { sufficient information provided to deter- } \\
\text { mine whether adequate sequence genera- } \\
\text { tion }\end{array}$ \\
\hline Allocation concealment (selection bias) & Unclear risk & No information provided \\
\hline $\begin{array}{l}\text { Blinding of participants and personnel } \\
\text { (performance bias) } \\
\text { All outcomes }\end{array}$ & High risk & $\begin{array}{l}\text { The intervention required active involve- } \\
\text { ment of the patient and healthcare profes- } \\
\text { sionals, so blinding not possible }\end{array}$ \\
\hline $\begin{array}{l}\text { Blinding of outcome assessment (detection } \\
\text { bias) } \\
\text { All outcomes }\end{array}$ & High risk & $\begin{array}{l}\text { Individualised 'theoretical' GAS scales were } \\
\text { created and scored for all participants by the } \\
\text { researcher and the occupational therapist of } \\
\text { the experimental groups (both unblinded). } \\
\text { The occupational therapist for the control } \\
\text { group not apparently involved in outcome } \\
\text { assessment }\end{array}$ \\
\hline $\begin{array}{l}\text { Incomplete outcome data (attrition bias) } \\
\text { All outcomes }\end{array}$ & Unclear risk & $\begin{array}{l}\text { No information provided about intention } \\
\text { to treat analysis. Three of } 27(11.1 \%) \text { par- } \\
\text { ticipants did not complete the study and } \\
\text { were excluded from analysis }\end{array}$ \\
\hline Selective reporting (reporting bias) & Unclear risk & $\begin{array}{l}\text { Unable to find a protocol for this study } \\
\text { published prior to it being conducted, so } \\
\text { unable to compare the outcomes reported } \\
\text { with those planned to be measured at the } \\
\text { outset }\end{array}$ \\
\hline Other bias & Unclear risk & $\begin{array}{l}\text { Management of risk for cross-group con- } \\
\text { tamination not adequately reported }\end{array}$ \\
\hline
\end{tabular}


Methods

Interventions
RCT

Setting: A hospital-based cardiac rehabilitation programme in Ontario and Quebec, Canada

Funding: Not reported. Completed as a PhD thesis

Recruitment (patients): Recruited from the cardiac rehabilitation programme run from hospital based rehabilitation centre. Potential participants were first identified by a nursing co-ordinator

Recruitment (healthcare providers): Not reported

Inclusion criteria (patients): 60 years of age or younger; discharged from cardiac bypass surgery two to three weeks prior to entry into the study; having medical clearance for returning to work within six months of discharge from hospital

Exclusion criteria (patients): Being clergy or members of a religious order, homemakers, students, or retirees

Consideration of people with comorbidities: Not reported

Patients: 68 participants randomised to study groups, 54 attended the first session, and 46 participants completed the study (91.3\% male). Mean age 48 (SD 7). Ethnicity not reported

Principle health problems: Recovering from cardiac bypass surgery

Treatment currently receiving: Not reported

Description of healthcare providers: Two providers delivered the intervention: a vocational counsellor and a 'researcher'. The vocational counsellor was also a doctoral student of clinical psychology. The study interventions were provided under conditions of clinical training and close clinical supervision. Demographic characteristics of the 'researcher' were unclear however

Study aim: To test the hypothesis that people recovering from cardiac bypass surgery would be a) more likely to return to work, b) work more, c) have higher life satisfaction, d) have higher job satisfaction, e) have higher satisfaction with health services, f) report greater improvements in self-efficacy, g) report greater improvements in self-determination, h) report greater improvements in commitment to work, and i) have less depression and anxiety if they participate in a group-based goal setting intervention than if they participate in watching and discussing educational videos about the workplace

Intervention: ( $\mathrm{n}=$ not reported, number completing intervention $=22$ ) Group-based goal setting. Participated in four group-based sessions with a work counsellor, in groups of one to four people in size (i.e. some one-on-one therapy was in fact offered). The sessions consisted of: completion of questionnaires about long-terms and two-month goals for return to work, provision of homework related to management of barriers to goal achievement, discussion of homework and other concerns related to goal achievement , and setting weekly goals (specific, challenging and self-relevant) to facilitate return to work. The counsellor helped participants revaluated and modify goals if they were deemed too difficult or too easy, too general, or not self-relevant

Control: ( $\mathrm{n}=$ not reported, number completing intervention $=24$ ) Video-based education and discussion about work management. Instead of goal setting discussions, these participants watched a humorous video concerning change, stress, stress management, and dealing with difficult people at work, followed by discussion of concerns related to the content of the videos facilitated by a vocational counsellor

Delivery: All participants contacted two to three weeks after hospital discharge for cardiac bypass surgery. All interventions offered over four sessions, but the total duration of these 
Iacovino 1997 (Continued)

sessions was not reported

Fidelity: Some variability was report in terms of the number of sessions that participants attended. Mean number of sessions per participants 3.09 (SD 0.92) for the goal setting group; and 3.21 (SD 0.88) for the video-watching group

Consumer involvement outside of the intervention: Not reported

Outcomes

Timing of outcomes: Three to six months after completion of the intervention

Return to work status

Percentage of eligible weeks worked

Self-efficacy

Intention to return to work

Commitment Scale of the Work Values Inventory

Conscientiousness Scale of the NEO-Five Factor Inventory

Client Motivation for Therapy Scale

Satisfaction with Life Scale

Zung Self-Rating Depression Scale

Job satisfaction

Goal setting characteristics

Comparison of interest: Collaborative goal setting plus strategies to enhance goal pursuit versus an attention control with no goal setting

Patient involvement in goal setting: Goals collaboratively set by the patient and healthcare professional

Family involvement in goal setting: None reported

Name of goal setting approach: No specifically named approach used

Development of a plan for goal pursuit: Strategies to achieve goals were discussed at the weekly meetings

Written copy of goals provided to patients: Patients wrote and kept a copy of their own goals

Individual versus group-based goal discussion: Individual or group-based

Level of goal difficulty: Challenging and difficult but attainable goals were emphasised Goal areas of focus: Work-related

Evaluation of patient goal commitment: Behavioural intentions towards to goal achievement were assessed using Likert-type questions

Goal reminders used: Patient were encouraged to reflect on their goals at each weekly meeting

Monitoring of progress towards goals: At each weekly meeting, patients self-rated their progress towards achieving their goals

Notes

Power calculation: Not reported

Risk of bias

\section{Bias}

Random sequence generation (selection Unclear risk bias)

\section{Authors' judgement}

\section{Support for judgement}

Participants '... were randomly assigned to goal-setting or video-tape treatment groups' (p.74). Insufficient information provided to determine whether adequate sequence generation 
Iacovino 1997 (Continued)

\begin{tabular}{|c|c|c|}
\hline Allocation concealment (selection bias) & Unclear risk & No information reported \\
\hline $\begin{array}{l}\text { Blinding of participants and personnel } \\
\text { (performance bias) } \\
\text { All outcomes }\end{array}$ & High risk & $\begin{array}{l}\text { The intervention required active involve- } \\
\text { ment of the patient and healthcare profes- } \\
\text { sionals, so blinding not possible }\end{array}$ \\
\hline $\begin{array}{l}\text { Blinding of outcome assessment (detection } \\
\text { bias) } \\
\text { All outcomes }\end{array}$ & Unclear risk & Insufficient information provided \\
\hline $\begin{array}{l}\text { Incomplete outcome data (attrition bias) } \\
\text { All outcomes }\end{array}$ & High risk & $\begin{array}{l}\text { Intention to treat analysis not reported. Of } \\
\text { the } 68 \text { participants randomly allocated to } \\
\text { study groups, } 22(32.4 \%) \text { did not complete } \\
\text { the study and were not included in the anal- } \\
\text { ysis }\end{array}$ \\
\hline Selective reporting (reporting bias) & Unclear risk & $\begin{array}{l}\text { Multiple outcomes measured. Unable to } \\
\text { find a protocol for this study published } \\
\text { prior to it being conducted, so unable to } \\
\text { compare the outcomes reported with those } \\
\text { planned to be measured at the outset }\end{array}$ \\
\hline Other bias & Low risk & No evidence of other sources of bias \\
\hline
\end{tabular}

\section{James 1993}

Methods

\section{RCT}

Setting: Not reported, but authors from a US university and a medical centre

Funding: Not reported

Recruitment (patients): Via local media and physician referrals

Recruitment (healthcare providers): Not reported

Inclusion criteria (patients): Diagnosis of migraine or tension-type headache as per the Headache Classification Committee of the International Headache Society; at least 18 years of age, without evidence of major psychotic or affective disorder, medical permission to participate

Exclusion criteria (patients): No further criteria reported

Consideration of people with comorbidities: Not reported

Participants

Patients: 48 participants enrolled. 33 participants completed the study (15.2\% male). Mean age 39 (SD 11). 100\% Caucasian

Principle health problems: Migraine or tension-type headache, with a mean duration of pain of 219 months (SD 141 months)

Treatment currently receiving: Cognitive behavioural therapy for chronic headaches Description of healthcare providers: Graduate students in clinical psychology

Interventions

Study aim: To compare of treatment outcome for patients with chronic headache pain who were given explicit goals for use of coping strategies versus instructions to use strategies for as long as possible (open group) 
Intervention (Cognitive behaviour therapy plus explicit goals): ( $=18)$ Cognitive behaviour therapy plus explicit, time-limited goals for use of pain coping strategies. A sixweek cognitive behaviour therapy programme consisting of weekly 90 minutes sessions, addressing nature of chronic headache pain, gate control theory, role of stressors in the pain response, coping versus catastrophising, developing and using appropriate images and self-talk, maintaining and generalising skills to other settings, plus 30 minutes of relaxation strategies, skills, practice. Oral and written instruction was also given regarding goals for use of pain coping strategies, specifically to a) practice coping strategies for 20 minutes per day, (b) take three 10-minute breaks each day to use strategies for coping with daily stressors, and (c) to use strategies for a period of 30 minutes when pain was experienced

Intervention (Cognitive behaviour therapy plus non-specific goals): $(n=19)$ Cognitive behavioural therapy without specific goals for use of pain coping strategies. A six-week cognitive behaviour therapy programme consisting of weekly 90 minutes sessions, as above. However, instead of specific goals for use of coping strategies, this group was given the following: oral and written instructions to (a) practice strategies as much and as often as possible; (b) use strategies as much as possible to cope with daily stressors; and (c) use the session-trained coping behaviours for as long as possible when they experience pain Control: $(n=7)$ Participants received delayed treatment, were given no instructions for coping, and were asked to continue to record headache activity, pain behaviour, and medication intake

Delivery: All treatment delivered over a six-week period

Fidelity: All treatment sessions audiotaped and scored on the Cognitive Therapy Rating Scale by one of the researchers (not otherwise involving in delivering treatment) to check fidelity

Consumer involvement outside of the intervention: Not reported

Outcomes

Goal setting characteristics
Timing of outcomes: Three weeks before and three weeks after the six-week intervention period

Pain intensity over a three-week period

Medication use (percentage of maximum recommended dose)

'Downtime', based on daily self-rating of the time spent in a supine position due to pain, excluding regular sleep time, averaged or a three-week period

'Coping time', based on self-rated time spent in active use of session-trained coping behaviours, calculated as a) average daily time practicing and using strategies to cope with stressors, and b) average daily time spent coping with pain

Pain Behaviour Questionnaire

Self-efficacy for pain

Global Severity Index, based on scores from a Symptom Checklist 90 revised

Sickness Impact Profile

Beck Depression Inventory

State Trait Anxiety Inventory

Cognitive Coping Index of the Cognitive Coping Strategies Inventory

Comparison of interest: Therapy plus prescribed goal setting (goals related to practice of strategies, not outcomes) versus therapy without goal setting versus no additional input Patient involvement in goal setting: None. Goals prescribed according to group allocation Family involvement in goal setting: None reported

Name of goal setting approach: No specifically named approach used 
Development of a plan for goal pursuit: Goals were about practice of coping strategies, so to a degree the plan for goal pursuit was implied within the goals

Written copy of goals provided to patients: Yes

Individual versus group-based goal discussion: Unclear

Level of goal difficulty: Not reported

Goal areas of focus: Implementation of coping strategies for management of pain

Evaluation of patient goal commitment: Not reported

Goal reminders used: Instructions regarding goals were provided at each treatment session

Monitoring of progress towards goals: Not reported

Notes Power calculation: Not reported

\section{Risk of bias}

\begin{tabular}{lll}
\hline Bias & Authors' judgement & Support for judgement
\end{tabular}

Random sequence generation (selection High risk bias)

'Subjects in the second and third recruitments were first blocked by headache diagnosis then randomly assigned within blocks to one of the groups. All subjects in the third recruitment were assigned to either goal or open conditions. Several individuals requested that they be assigned to different groups because of a preexisting relationship with other group members or a conflict with their work schedule' (p.310)

\begin{tabular}{|c|c|c|}
\hline Allocation concealment (selection bias) & High risk & See above \\
\hline $\begin{array}{l}\text { Blinding of participants and personnel } \\
\text { (performance bias) } \\
\text { All outcomes }\end{array}$ & Unclear risk & $\begin{array}{l}\text { The intervention required active involve- } \\
\text { ment of the patient and healthcare profes- } \\
\text { sionals, so blinding not possible. However, } \\
\text { 'Therapists were aware of the differences in } \\
\text { the instructional sets for groups but were } \\
\text { not informed of the experimental hypothe- } \\
\text { ses' (p.310) }\end{array}$ \\
\hline
\end{tabular}

Blinding of outcome assessment (detection High risk Outcome assessment not blinded bias)

All outcomes

Incomplete outcome data (attrition bias) High risk All outcomes

Selective reporting (reporting bias)
High risk
'Fourty-eight subject attended the initial orientation sessions; 13 were lost to followup' (i.e. $27.1 \%$ attrition)

'Two additional subjects were identified as sample outliers and were subsequently dropped from analysis' (p.308). Unable to find a protocol for this study published 
prior to it being conducted, so unable to compare the outcomes reported with those planned to be measured at the outset

Other bias $\quad$ Unclear risk

Therapist adherence to treatment protocols was evaluated with the Cognitive Therapy Rating Scale revealing '...no significant differences between goal and open groups in (a) degree of therapists adherence to general treatment protocol, (b) degree of therapist adherence to the correct instructional set, and (c) level of group difficulty' (p.312) . Multiple outcome measures were used, with no primary outcome nominated, raising the risk of positive differences between the groups appearing by chance

Jonsdottir 2012

Methods

Cluster-RCT. Patients clustered by therapist

Setting: An inpatient rehabilitation unit, Switzerland

Funding: Not reported

Recruitment (patients): Not reported

Recruitment (healthcare providers): Not reported

Inclusion criteria (patients): All neurological patients admitted to the rehabilitation unit with Mini Mental State Exam score of 24 or greater were recruited to the study (author communication)

Exclusion criteria (patients): Not reported

Consideration of people with comorbidities: Not reported

Participants

Patients: 8 participants. Demographic data not reported

Principle health problems: Neurological disorders (75\% multiple sclerosis; $25 \%$ stroke) Treatment currently receiving: Fifteen rehabilitation session over a 3-4 week period, the content of which was not reported

Description of healthcare providers: Not reported

Interventions

Study aim: To evaluate the use of the ICF tools in the REHAB cycle in improving health outcomes for people with multiple sclerosis and stroke

Intervention: $(\mathrm{n}=4)$ Goal-directed therapy. Participants were classified using the appropriate ICF core set. Individual rehabilitation goals were agreed on by the researcher, participant, and therapist. The 'Tool of REHAB Cycle' was used to plan intervention. This group differed from the control group because this group 'was treated with the objective of achieving the set goals'

Control: $(n=4)$ Usual care. Participants were classified using the appropriate ICF core set and had a REHAB cycle plan developed, but were treated by a different group of therapist who were not informed of the goals, and who followed 'usual care rehabilitation', with no restriction or direction place on them regarding goal setting for rehabilitation. 'Systematic goal setting in accordance with the patient was not part of [the control therapists] usual

Goal setting and strategies to enhance goal pursuit for adults with acquired disability participating in rehabilitation (Review) 
Jonsdottir 2012 (Continued)

routine' (author communication)

Delivery: All goal setting and intervention delivered over a 3-4 week period

Fidelity: Not reported

Consumer involvement outside of the intervention: Not reported

\begin{tabular}{ll} 
Outcomes & $\begin{array}{l}\text { Timing of outcomes: After } 15 \text { sessions over a 3-4 weeks period } \\
\text { Goal achievement }\end{array}$ \\
\hline Goal setting characteristics & $\begin{array}{l}\text { Comparison of interest: Collaborative goal setting versus usual care } \\
\text { Patient involvement in goal setting: Goals collaboratively set by the patient and healthcare } \\
\text { professional }\end{array}$ \\
Family involvement in goal setting: Not reported \\
Name of goal setting approach: No specifically named approach used \\
Development of a plan for goal pursuit: Goals used to plan rehabilitation \\
Written copy of goals provided to patients: Not reported \\
Individual versus group-based goal discussion: Individual \\
Level of goal difficulty: Not reported \\
Goal areas of focus: Linked to the ICF Core Sets \\
Evaluation of patient goal commitment: Not reported \\
Goal reminders used: Not reported \\
Monitoring of progress towards goals: Not reported
\end{tabular}

Notes

Power calculation: The research was intended as pilot study, so no power calculation was reported (author communication)

Risk of bias

\begin{tabular}{|c|c|c|}
\hline Bias & Authors' judgement & Support for judgement \\
\hline $\begin{array}{l}\text { Random sequence generation (selection } \\
\text { bias) }\end{array}$ & Unclear risk & $\begin{array}{l}\text { 'The coordinator of rehabilitation ran- } \\
\text { domly assigned the patients (as they ar- } \\
\text { rived) to therapists' (author communica- } \\
\text { tion) }\end{array}$ \\
\hline Allocation concealment (selection bias) & Low risk & $\begin{array}{l}\text { 'The coordinator [when assigning patients } \\
\text { to therapists] was completely blind to } \\
\text { which therapists were treating patients with } \\
\text { ICF goal outcome and which were giving } \\
\text { usual care' (author communication) }\end{array}$ \\
\hline
\end{tabular}

Blinding of participants and personnel High risk (performance bias)

All outcomes
Patients were clustered by therapist. 'Nothing was asked of the control group therapists, and they were unaware of the patients being part of a treatment study' (author communication). However, therapists and patients in the intervention group were required to be actively involved in the intervention protocol, so blinding was not possible 
Jonsdottir 2012 (Continued)

\begin{tabular}{|c|c|c|}
\hline $\begin{array}{l}\text { Blinding of outcome assessment (detection } \\
\text { bias) } \\
\text { All outcomes }\end{array}$ & Low risk & $\begin{array}{l}\text { The therapist who completed the ICF clas- } \\
\text { sification, initial goal setting, and goal eval- } \\
\text { uation with all patients was blinded to } \\
\text { group allocation (author communication) }\end{array}$ \\
\hline
\end{tabular}

Incomplete outcome data (attrition bias) Low risk

All outcomes

No evidence of attrition, with all participants accounted for in the analysis

Selective reporting (reporting bias) Unclear risk

Unable to find a protocol for this study published prior to it being conducted, so unable to compare the outcomes reported with those planned to be measured at the outset

Other bias

Low risk

Cross-group contamination was managed by a cluster-RCT design. 'There were two groups of therapists in two different locations of the rehabilitation center providing treatment, however the characteristics of the two groups was similar in terms of experience with neurological rehabilitation and years in service' (author communication)

\section{LaFerriere 1978}

\begin{tabular}{ll} 
Methods & RCT \\
Setting: An outpatient unit for people with mental health conditions in Michigan, USA \\
Funding: Not reported \\
Recruitment (patients): Recruited from existing clients of the outpatient unit \\
Recruitment (healthcare providers): Recruited from existing staff member of the outpa- \\
tient unit \\
Inclusion criteria (patients): Other than being clients of the mental health outpatient \\
unit in question, inclusion criteria were not report \\
Exclusion criteria (patients): No further criteria reported \\
Consideration of people with comorbidities: Not reported \\
\hline Participants \\
$\begin{array}{l}\text { Patients: } 65 \text { participants (24.6\% male). } 100 \% \text { Caucasian } \\
\text { Principle health problems: Mental health disorders; specific diagnoses not reported } \\
\text { Treatment currently receiving: Typically clients in this setting were seen on an individual } \\
\text { basis for five to six sessions } \\
\text { Description of healthcare providers: Before the study, '11 therapists in the outpatient } \\
\text { unit participated in two sessions of orientation and practice using GAS... Therapists } \\
\text { in the unit typically have undergraduate or master's degrees in counseling or social work } \\
\text { and less than } 5 \text { years counseling experience' (p. 273) }\end{array}$
\end{tabular}

Goal setting and strategies to enhance goal pursuit for adults with acquired disability participating in rehabilitation (Review) 

by means of GAS would when compared to the control group: (a) Be perceived by their therapists as more motivated to change in therapy; (b) Be perceived by their therapists as having changed more as a result of therapy; (c) Rate themselves as more motivated to change; (d) Rate themselves as having changed more as a result of therapy; (e) Exhibit better adjustment as measured by standardized tests of anxiety, depression, and selfesteem' (p.272)

Intervention: $(\mathrm{n}=34)$ Goal setting group. GAS was used in the first two therapy sessions to assist clients and therapists to mutually agree upon goals for therapy. At least three goals were to be established and written in GAS format for each participant. The standard GAS approach was modified to a three-point scale however. Therapists were to refer to the goals throughout the client's therapy

Control: $(n=31)$ Participants in this group did not engage with the goal setting process. Therapists followed usual practice

Delivery: Goal setting and therapy were delivered over a series of sessions. The usual number of sessions was reported to be five to six per patient, but in this study participants were reported as receiving more than six therapy sessions on average

Fidelity: Interventions were monitored, but deviations from the study protocol were managed by dropping the participants from the analysis. In particular, eight goal setting group clients were dropped from the analysis because 'therapists did not administer the prescribed GAS treatment, i.e., written goals by the end of the second therapy session' (p.275)

Consumer involvement outside of the intervention: Not reported

A measure of trait anxiety 'using a random sample of half of the items of the Welsh Anxiety Scale'

A questionnaire measuring (on five-point scales): satisfaction with counselling, self-reported motivation, self-reported change, client's use of goals, client's awareness of specific goals (the number of specific goals recalled by the clients when asked)

Therapist-rated perception of: client motivation for change (3-point scale), client change (3-point scale), therapist use of goals in the therapy process (4-point scale)

Comparison of interest: Collaborative goal setting versus usual care with no structured goal setting

Patient involvement in goal setting: Goals collaboratively set by the patient and healthcare professional

Family involvement in goal setting: None reported

Name of goal setting approach: GAS

Development of a plan for goal pursuit: Not reported

Written copy of goals provided to patients: Not reported

Individual versus group-based goal discussion: Individual

Level of goal difficulty: Not reported

Goal areas of focus: Not reported

Evaluation of patient goal commitment: Not reported

Goal reminders used: Not reported 
LaFerriere 1978 (Continued)

Monitoring of progress towards goals: Not reported

\begin{tabular}{|c|c|c|}
\hline Notes & Power calculation: Not reported & \\
\hline \multicolumn{3}{|l|}{ Risk of bias } \\
\hline Bias & Authors' judgement & Support for judgement \\
\hline $\begin{array}{l}\text { Random sequence generation (selection } \\
\text { bias) }\end{array}$ & Low risk & $\begin{array}{l}\text { 'A random number table was then used to } \\
\text { decide whether the client was to be a GAS } \\
\text { or control client' (p.273) }\end{array}$ \\
\hline Allocation concealment (selection bias) & Low risk & $\begin{array}{l}\text { 'Secretaries assigned clients to groups when } \\
\text { they call to make their initial appointments' } \\
\text { (author communication) }\end{array}$ \\
\hline
\end{tabular}

Blinding of participants and personnel High risk (performance bias)

All outcomes

The intervention required active involvement of the patient and healthcare professionals, so blinding not possible

Blinding of outcome assessment (detection High risk bias)

All outcomes

Outcome data were gathered by researchers who were not involved in setting goals or providing therapy to the participants in the study. It was reported by the authors that 'I believe [outcome assessors] were blinded on the standardized measures but probably saw the differences in how both groups set their goals for the subjective follow up questions' (author communication). Many outcomes were based on self-report of participants not blinded to group allocation

Incomplete outcome data (attrition bias) High risk All outcomes

Of the 65 participants who enrolled in the study, 33 (50.8\%) were dropped from the analysis. 'Eight GAS clients were dropped from the analysis because therapists did not administer the prescribed GAS treatment, i.e. written goals by the end of the second therapy session. An additional 25 clients could not be contacted for the posttest assessment' (p. 275)

Selective reporting (reporting bias) Unclear risk

Unable to find a protocol for this study published prior to it being conducted, so unable to compare the outcomes reported with those planned to be measured at the outset

Goal setting and strategies to enhance goal pursuit for adults with acquired disability participating in rehabilitation (Review) 
LaFerriere 1978 (Continued)

$\begin{array}{ll}\text { Other bias Low risk } & \text { L }\end{array}$

Mann 1987
Risk of cross-group contamination low because participants in the groups 'would not interact typically as clients [and] were seen individually in a community mental health setting in a large metro area' (author communication)

\begin{tabular}{|c|c|}
\hline Methods & $\begin{array}{l}\text { RCT } \\
\text { Setting: A hypertension clinic in a university teaching hospital in Nova Scotia, Canada } \\
\text { Funding: Supported by the Canadian and Nova Scotia Heart Foundations } \\
\text { Recruitment (patients): Recruiting from patients referred by a family physician to the } \\
\text { hypertension clinic } \\
\text { Recruitment (healthcare providers): Not reported } \\
\text { Inclusion criteria (patients): adults with hypertension, managed medically; sitting dias- } \\
\text { tolic blood pressure of } 91 \text { to } 104 \mathrm{mmHg} \text {, on two occasions; taking none, one or more } \\
\text { antihypertensive medications, with those on medication judged to be able to remain on } \\
\text { the same regimen for the study duration; able to read English at grade } 7 \text { to } 9 \text { level; no } \\
\text { complicating medical conditions, or contraindications to participation } \\
\text { Exclusion criteria (patients): No further criteria reported } \\
\text { Consideration of people with comorbidities: Not reported }\end{array}$ \\
\hline Participants & $\begin{array}{l}\text { Patients: } 66 \text { participants ( } 37.5 \% \text { male). Mean age } 48 \text { (SD not reported). Range } 18 \text { to } \\
70 \text { years. Ethnicity not reported } \\
\text { Principle health problems: Hypertension } \\
\text { Treatment currently receiving: Not reported } \\
\text { Description of healthcare providers: Interventions lead by the researchers }\end{array}$ \\
\hline Interventions & $\begin{array}{l}\text { Study aim: To these the hypotheses that '(1) Hypertensive adults who receive a task- } \\
\text { centered instructional program will achieve and maintain significantly greater increases } \\
\text { in knowledge related to reducing dietary sodium, and significantly greater reduction in } \\
\text { dietary sodium intake than those who receive no specific instruction on dietary sodium' } \\
\text { and that'(2) Hypertensive adults who receive a task-centred instructional program plus } \\
\text { task-relevant goal setting and self-monitoring will achieve and maintain significantly } \\
\text { greater increases in knowledge related to reducing dietary sodium, and significantly } \\
\text { greater reduction in dietary sodium intake than those receiving the task-centred instruc- } \\
\text { tional program alone, or those receiving no specific instruction on dietary sodium } \\
\text { Intervention (Instruction): ( } \mathrm{n}=\text { not reported, number completing intervention = 19) } \\
\text { Task-centred instruction. An instructional programme that included: instructional ob- } \\
\text { jectives, behavioural assessment, instructional procedures, and performance assessment, } \\
\text { with a focus on tasks which the individual must accomplish to achieve and maintain } \\
\text { reduced dietary sodium intake, delivered on a one-to-one basis over six weekly sessions. } \\
\text { An instruction booklet was also provided } \\
\text { Intervention (Instruction plus goal setting): (n = not reported, number completing in- } \\
\text { tervention = 19) Task-centred instruction plus task-relevant goal setting and self-moni- } \\
\text { toring. An instructional programme as above, plus the addition of collaborative goal-set- } \\
\text { ting and goal-relevant self-monitoring. Each participant had one goal per week, selecting }\end{array}$ \\
\hline
\end{tabular}

Goal setting and strategies to enhance goal pursuit for adults with acquired disability participating in rehabilitation (Review) 
from a set of categories (all related to changing eating habits, cook habits, or shopping habits) Goals were stated in terms of the behaviours to be changed. No specific sodium goal was set, although all participants in the two intervention groups were advised that 2-3 g of sodium daily was a recommend range. If one goal, one week was achieved, another would be set

Control: $(\mathrm{n}=$ not reported, number completing intervention $=18)$ Six weekly visits as per the other groups, but without provision of the instruction or goal setting. What was provided instead during these visits was not reported

Delivery: All goal setting and therapy delivered over six weeks

Fidelity: Not reported

Consumer involvement outside of the intervention: Not reported

Outcomes

Goal setting characteristics
Timing of outcomes: Immediately following the 6-week therapy programme and three months later

24-hour self-reported dietary sodium intake

24-hour urinary sodium scores

Criterion-referenced achievement test

Systolic BP

Diastolic BP

Comparison of interest: Patient training/education versus patient training/education plus goal setting versus attention control with no goal setting

Patient involvement in goal setting: Patients selected one goal per week from a prescribed list of goal topics related to dietary behaviour

Family involvement in goal setting: None reported

Name of goal setting approach: No specifically named approach used

Development of a plan for goal pursuit: All goals related directly to training in selfmanagement provided to the patients

Written copy of goals provided to patients: Not reported

Individual versus group-based goal discussion: Individual

Level of goal difficulty: Not reported

Goal areas of focus: Dietary behaviour

Evaluation of patient goal commitment: Not reported

Goal reminders used: Goals were reviewed weekly and new goals set if the previous goal had been achieved or were revised if they had not been achieved

Monitoring of progress towards goals: Goal progress was self-monitored by patient using forms for self-monitoring of dietary behaviour

\section{Risk of bias}

\section{Bias}

Random sequence generation (selection Unclear risk bias)
Authors' judgement

Support for judgement

Participants were'randomly assigned to one of three groups' (p.57). Insufficient information provided to determine whether adequate sequence generation 


\begin{tabular}{|c|c|c|}
\hline Allocation concealment (selection bias) & Unclear risk & No information provided. \\
\hline $\begin{array}{l}\text { Blinding of participants and personnel } \\
\text { (performance bias) } \\
\text { All outcomes }\end{array}$ & High risk & $\begin{array}{l}\text { The intervention required active involve- } \\
\text { ment of the patient and healthcare profes- } \\
\text { sionals, so blinding not possible }\end{array}$ \\
\hline $\begin{array}{l}\text { Blinding of outcome assessment (detection } \\
\text { bias) } \\
\text { All outcomes }\end{array}$ & Unclear risk & $\begin{array}{l}\text { Insufficient information provided to de- } \\
\text { termine whether the outcome assessor was } \\
\text { blinded to group allocation or not }\end{array}$ \\
\hline $\begin{array}{l}\text { Incomplete outcome data (attrition bias) } \\
\text { All outcomes }\end{array}$ & High risk & $\begin{array}{l}\text { Of the } 62 \text { participant who enrolled in } \\
\text { the study, } 11(17.7 \%) \text { were dropped from } \\
\text { the analysis. This included 'one person } \\
\text { [who] withdrew voluntarily from each } \\
\text { study group; a fourth [who] had major } \\
\text { surgery... three [who] failed to collect valid } \\
24 \text {-hour urine specimens, and three [who] } \\
\text { required a change in medication during the } \\
\text { study' (p.62) }\end{array}$ \\
\hline Selective reporting (reporting bias) & Unclear risk & $\begin{array}{l}\text { Unable to find a protocol for this study } \\
\text { published prior to it being conducted, so } \\
\text { unable to compare the outcomes reported } \\
\text { with those planned to be measured at the } \\
\text { outset }\end{array}$ \\
\hline Other bias & Low risk & No evidence of other sources of bias \\
\hline
\end{tabular}


Methods

Participants
RCT

Setting: Residential and non-residential neurorehabilitation services, New Zealand

Funding: Health Research Council of New Zealand

Recruitment (patients): People with traumatic brain injury recruited from one of three neurorehabilitation services

Recruitment (healthcare providers): Not reported

Inclusion criteria (patients): moderate to severe traumatic brain injury with disabling consequence (indicated by post-traumatic amnesia of over one hour and moderate disability on the Extended Glasgow Outcome Scale); receiving rehabilitation from a neurorehabilitation service; agreement from their keyworker to deliver the intervention with the support of the researchers

Exclusion criteria (patients): persistent coma (indicated by Glasgow Coma Scale less than 8 on screening); cognitive or communicative deficit so severe as to prevent participation in the study; unstable medical health conditions precluding participation in the rehabilitation

Consideration of people with comorbidities: Not reported

Patients: 34 participants $(79.4 \%$ male). Mean age 29 year for the Intervention group (GMT); 28 years for Intervention group (IOGT); 40 years for the Control group (SD not reported). Range 19 to 68 years

52.9\% 'New Zealander', 8.8\% NZ European, 29.4\% NZ Maori, 8.8\% Pacific Islander Principle health problems: Traumatic brain injury. Range of time since injury: 1-31 years Treatment currently receiving: Community-based long-term management and rehabilitation

Description of healthcare providers: Delivered by healthcare providers trained in the interventions with support of the research team

Interventions

Study aim: A pilot RCT with the primary objective of determining the acceptability of two goal setting approaches designed to improve skills in self-regulation, for use with people with traumatic brain injury

Intervention (GMT): $(\mathrm{n}=12)$ GMT. Individual meetings with a keyworker once a week for six weeks (over an 8-week period to allow for missed appointments). Sessions involved identification and documentation of a goal, identification of steps required to achieve that goals, verbal rehearsal of the steps, monitoring to prevent experiences of goal failure, and practicing of the steps once no errors in performance were noted

Intervention (IOGT): $(\mathrm{n}=10)$ Identity Oriented Goal Training (IOGT). Individual meetings with keyworker once a week for six weeks (over an 8-week period to allow for missed appointments). Sessions involved use of an 'identity map' as a tool for identification and articulation of goals that would help participants connect with and progress towards a meaningful, higher order state

Control: $(n=12)$ Usual care. No interaction with their keyworker in addition to their usual rehabilitation plan, except to complete Goal Attainment Scaling (GAS) for outcome evaluation

Delivery: All goal setting and therapy was delivered over an eight-week period

Fidelity: A detailed protocol for each intervention was developed and used to guide intervention sessions

Qualitative data on barriers to implementation of the intervention were reported. It was noted that GAS, while intended as an outcome measure in this study, 'in fact seem[ed] to act as an intervention on its own with negotiation around goals occurring within the 
McPherson 2009 (Continued)

process of conducting the Goal Attainment Scale' (p. 307)

Consumer involvement outside of the intervention: Not reported

Outcomes

Timing of outcomes: On completion of the eight-week intervention, and at a threemonth follow-up

GAS

Goal setting characteristics

Comparison of interest: Goal Management Training versus Identity Oriented Goal Training versus no additional input

Patient involvement in goal setting: Goals collaboratively set by the patient and healthcare professional

Family involvement in goal setting: None reported

Name of goal setting approach: GMT and Identity Oriented Goal Training

Development of a plan for goal pursuit: Both goal setting approaches involved development of a plan for goal achieved, but involved different methods for doing so

Written copy of goals provided to patients: A written copy of the goals and goal plan was given to all participants (author communication)

Individual versus group-based goal discussion: Individual

Level of goal difficulty: GMT involved strategy for errorless learning. So GMT could potentially have involved challenging goals, but the approach involved ensuring prevention of goal failure. Goal difficult was not commented on in the Identity Oriented Goal Training approach

Goal areas of focus: Not restricted to any particular topics

Evaluation of patient goal commitment: Not reported

Goal reminders used: Weekly meeting were used to discuss goals and strategies for goal pursuit with patients

Monitoring of progress towards goals: Discussed in the weekly meetings

Notes

Power calculation: Not attempted as the study was intended as a pilot

Risk of bias

Bias

Authors' judgement

Support for judgement

Random sequence generation (selection Low risk bias)

'...participants were randomized to one of three intervention groups' (p. 298). The randomisation sequence was computer generated (author communication)

Allocation concealment (selection bias) Low risk

'Block randomization was used to achieve balanced allocation to interventions and a third-party randomization schedule was generated by an independent researcher (MW) not involved in intervention delivery and sent to a second independent researcher who managed allocation' (p.298)

Goal setting and strategies to enhance goal pursuit for adults with acquired disability participating in rehabilitation (Review) 
McPherson 2009 (Continued)

\begin{tabular}{l|l|l}
$\begin{array}{l}\text { Blinding of participants and personnel } \\
\text { (performance bias) } \\
\text { All outcomes }\end{array}$ & High risk & $\begin{array}{l}\text { The intervention required active involve- } \\
\text { ment of the patient and healthcare profes- } \\
\text { sionals, so blinding not possible }\end{array}$ \\
\hline $\begin{array}{l}\text { Blinding of outcome assessment (detection } \\
\text { bias) } \\
\text { All outcomes }\end{array}$ & Unclear risk & $\begin{array}{l}\text { Goal attainment scaling data were gathered } \\
\text { by a research assistant blinded to group al- } \\
\text { location, but with involvement from the } \\
\text { participant and their therapists (who were } \\
\text { not blinded) for the scoring of GAS goals } \\
\text { (author communication) }\end{array}$ \\
\hline
\end{tabular}

Incomplete outcome data (attrition bias) High risk

All outcomes

Of the 34 participants originally enrolled, goal attainment scaling data were available for 25 participants (25.7\% attrition) postintervention and for 22 participants (35. $3 \%$ attrition) at the three month follow-up

Selective reporting (reporting bias) Unclear risk

No protocol was published for this study prior to it being conducted, so unable to compare the outcomes reported with those planned to be measured at the outset

Other bias $\quad$ Unclear risk

As this was a pilot study, the authors looked for, and noted, some occasions where crossgroup contamination was potentially a risk, and used this information for the development of more comprehensive procedures to minimise this risk in a future fully-powered trial (author communication)

Miller 2012

Methods

RCT

Setting: A university research centre, USA

Funding: National Center for Research Resources

Recruitment (patients): 'recruited through classified advertisements, employee newsletters, health fairs, medical practices, neighbourhood health centres, and flyers' (p.85)

Recruitment (healthcare providers): Not reported

Inclusion criteria (patients): diagnosis of type 2 diabetes for more than one year, glycosylated haemoglobin (A1c) value $\geq 7 \%$, not on insulin therapy

Exclusion criteria (patients): Mini Mental Status Exam score of less than 20, prior instruction in glycaemic index

Consideration of people with comorbidities: Not reported

Participants

Patients: 46 participants enrolled. 35 participants completed the intervention $(34.3 \%$ male). Mean age 52.6 (experimental group); 49.6 years (control group) (SD 5.9 experimental group; 6.7 control group). $88.6 \%$ Caucasian; $11.4 \%$ non-Caucasian Principle health problems: Type 2 diabetes

Goal setting and strategies to enhance goal pursuit for adults with acquired disability participating in rehabilitation (Review) 
Miller 2012 (Continued)

Treatment currently receiving: Five weeks of dietary training

Description of healthcare providers: A research dietician. Demographics on the health provider not reported

Interventions
Study aim: To test the hypothesis that a specific difficult goal related to improved dietary behaviour would result in greater improvements in self-efficacy and greater consumption of desirable, lower glycaemic index foods than a specific easier goal in people needing improve dietary regulation for type 2 diabetes

Intervention: ( $\mathrm{n}=$ not reported, number completing intervention: 20) Specific difficult dietary goal. Attended a five-week nutrition intervention provided by one dietician (prior to randomisation), then set goal by a second dietician to consume eight servings per day of lower glycaemic index foods. Plans to target dietary requirements were also developed with this second dietician. Participants were asked to self-monitor their diet and blood glucose at least four days per week during the following eight-week period. Met with the second dietician once more midway through the eight-week period to review the participant's progress. This meeting included discussion of self-reported goal achievement, barriers to goal achievement, and strategies

Control: ( $\mathrm{n}=$ not reported, number completing intervention $=15)$ Specific easier dietary goal. As for the intervention group but set a goal by the second dietician to achieve six servings per day of lower glycaemic index foods

Delivery: The intervention was delivered over an eight-week period

Fidelity: Not reported

Consumer involvement outside of the intervention: Not reported

\footnotetext{
Outcomes

Outcomes

Goal setting characteristics

Timing of data collection: baseline, following the five-week dietary intervention (prior to randomisation), on completion of the goal assignment appointment, and following the eight-week monitoring period

Glycaemic index derived from a self-reported 4-day food record

Number of servings of lower glycaemic index foods consumed derived from a selfreported 4-day food record

Perceived goal difficulty, commitment, and satisfaction

Modifiable Physical Activity Questionnaire

Self-efficacy (for consuming lower glycaemic index foods) with three subscales: glycaemic index efficacy (confidence or choosing and preparing lower glycaemic index foods), goal difficulty (confidence for consuming lower glycaemic index foods), and negative food selection (difficulty in choosing lower glycaemic index foods)

Comparison of interest: Prescribed, specific, difficult goals versus prescribed, specific, easier goals

Patient involvement in goal setting: None. Goals prescribed according to group allocation Family involvement in goal setting: None reported

Name of goal setting approach: Approach based on Locke and Latham's Goal Setting Theory

Development of a plan for goal pursuit: Plans to target dietary requirements were developed for all patients

Written copy of goals provided to patients: Not reported

Individual versus group-based goal discussion: Individual

Level of goal difficulty: The study involved comparing the setting difficult goals with the setting of easier goals
} 
Goal areas of focus: Dietary behaviour

Evaluation of patient goal commitment: 'Goal commitment was assessed with a previously validated questionnaire that included both positively and negatively stated items regarding participants' determination to achieve the goal' (p.86). Goal commitment measured following goal assignment and at the end of the study

Goal reminders used: Midway through the intervention period, the participants met again with a therapist to individually discuss their goals and goal progress

Monitoring of progress towards goals: In addition to the midway progress meeting with a therapist, participants were also asked to self-monitor their diet and blood glucose at least four days per week. Participant were provide training in undertaking this selfmonitoring. They were asked to return their self-monitoring records to the therapist weekly, who reviewed them and provided standardised feedback

\section{Risk of bias}

\begin{tabular}{l|ll}
\hline Bias & Authors' judgement & Support for judgement \\
\hline $\begin{array}{l}\text { Random sequence generation (selection } \\
\text { bias) }\end{array}$ & Low risk & $\begin{array}{l}\text { 'Randomization assignment was deter- } \\
\text { mined using randomization software...' (p. } \\
85)\end{array}$ \\
\hline $\begin{array}{l}\text { Allocation concealment (selection bias) } \\
\text { Low risk }\end{array}$ & Lon & 'Randomization assignment was... placed \\
\hline
\end{tabular}

Blinding of participants and personnel High risk (performance bias)

All outcomes

Blinding of outcome assessment (detection High risk bias)

All outcomes
The intervention required active involvement of the patient and healthcare professionals, so blinding not possible

'The study dietitian [who coordinated the data collection] was blinded to goal assignment' (p.85), however outcomes related to dietary intake were entirely based on patient self-report, who were not blinded to group allocation. Other outcomes (e.g. physical activity and self-efficacy) were also back on patient self-report

Incomplete outcome data (attrition bias) Unclear risk All outcomes
Of the 46 participants who originally enrolled in the study, five dropped out prior to randomisation, and six of the remaining 41 randomised participants (14.6\%) dropped out after randomisation but before final data collection, and were excluded from analysis (author communication) 
Miller 2012 (Continued)

\begin{tabular}{|l|l|l|l}
\hline Selective reporting (reporting bias) & Unclear risk & $\begin{array}{l}\text { Unable to find a protocol for this study } \\
\text { published prior to it being conducted, so } \\
\text { unable to compare the outcomes reported } \\
\text { with those planned to be measured at the } \\
\text { outset }\end{array}$ \\
\hline
\end{tabular}
outset

Other bias

Low risk

No evidence of other sources of bias

O'Brien 2013

Methods

RCT

Setting: A University exercise laboratory, New Zealand

Funding: New Zealand Manipulative Physiotherapists Association

Recruitment (patients): Not reported

Recruitment (healthcare providers): Not reported

Training/support: Not reported

Inclusion criteria (patients): People with hip and/or knee joint osteoarthritis according to the American College of Rheumatology classification criteria (radiographic evidence of osteoarthritic changes, joint pain on most days of the last month, plus three of the following: age 50 years or older; morning joint stiffness longer than 30 minutes; crepitus; bony tenderness; bony enlargement; and no palpable warmth); good command of English language; able to undertake exercise

Exclusion criteria (patients): Already receiving physiotherapy; having a disorder or illness that prevented exercise

Comorbidities: Not reported

Participants

Patients: 27 participants (40.7\% male). Mean age Mean age 63.3 (experimental group); 63.7 years (control group) (SD 10.4 experimental group; 11.3 control group). Ethnicity not reported

Principle health problems: Osteoarthritis (22.2\% hip; $74.1 \%$ knee OA; 3.7\% both). Time since diagnosis of osteoarthritis - 41.0 months experimental group (SD 8.5); 76.

7 months control group (47.7)

Treatment currently receiving: Gym and home-based exercise

Description of healthcare providers: A physiotherapy researcher delivered the goal setting.

A research assistant delivered the exercise classes

Interventions

Study aim: To test the hypothesis that participants with osteoarthritis who received an action and coping plans (based on goal setting) would have higher levels of self-efficacy, higher treatment adherence, and better function following an exercise programme, than those who did not receive an action and coping plan

Intervention: $(\mathrm{n}=10)$ Exercise plus action coping plans. Exercise consisted of three sessions per week of group-based activity for 12 weeks, plus a home-based walking and stretching programme, with close supervision during first four weeks and minimal supervision over last eight weeks. All participants taught to perform the exercises correctly, and encouraged to apply maximal effort to each exercise. Exercises included a resistancebased circuit programme, with resistance increased as ability improved. Home activity was to be undertaken twice weekly, and included a 20 min walk plus stretches for the lower limb. The action coping plan consisted of setting a realistic functional goal that

Goal setting and strategies to enhance goal pursuit for adults with acquired disability participating in rehabilitation (Review) 
O'Brien 2013 (Continued)

the participant wanted to achieve at the end of the 12-week exercise programme, plus completion of an action plan (when, where, how, and with whom they were going to undertake the home-based walking, home-based stretching, and class-based exercise programme) in order to achieve these goals, and completion of a coping plan, identifying obstacles to goal attainment and how to prevent them

Control: $(n=10)$ Exercise without action coping plans or any alternative goal setting. The exercise programme was the same in content, duration and intensity as for the intervention group

Delivery: All exercises delivered over 12 weeks

Fidelity: Not reported

Consumer involvement outside of the intervention: None reported

Outcomes

Timing of outcomes: Collected at the end of the 12-week exercise programme Sport injury Rehabilitation Adherence Scale (SIRAS)

Adherence - attendance of group classes and programme completion

Adherence - home-based; self-report on 5-point Likert scale indicating degree of adherence to exercise recommendations for home-exercise (for walking and stretching programmes)

Timed Up and Go

10 meter Walk Test

Step Test

Six minute Walk Test

ADL subscale of the Lower Limb Task Questionnaire

Goal setting characteristics

Comparison of interest: Goal setting with or without strategies to enhance goal pursuit versus no goal setting

Patient involvement in goal setting: Goal were collaboratively set and prioritised by the patient and therapist

Family involvement in goal setting: None reported

Name of goal setting approach: No specifically named approach used

Development of a plan for goal pursuit: A written plan for goal pursuit was developed in collaboration by the patient and therapist

Written copy of goals provided to patients: A copy of the action and coping plan, with goals, was given to the patients

Level of goal difficulty: An emphasis was placed on setting realistic goals

Goal areas of focus: Goal were set in the area of functional performance

Evaluation of patient goal commitment: Action and coping plans were signed and dated by the participant, but patient commitment to goal achievement was not reported

Goal reminders used: None reported

Monitoring of progress towards goals: None reported

Notes

Risk of bias

Bias

Authors' judgement

Support for judgement

Goal setting and strategies to enhance goal pursuit for adults with acquired disability participating in rehabilitation (Review)

Copyright @ 2015 The Cochrane Collaboration. Published by John Wiley \& Sons, Ltd. 
O’Brien 2013 (Continued)

Random sequence generation (selection Low risk bias)

Allocation concealment (selection bias) Low risk
'Participants were randomly assigned to either the intervention... or control... group with the use of a computer-generated random number table' (p. 49)

'I was blinded to potential participants group allocation until they were enrolled in the study. The number table was generated by my supervisor and given to my receptionist. When I recruited someone to the study they contacted my receptionist. She booked them into the study and added their name to the participants list (which generated their participant number) and then checked the participant number against the random number table. If they were assigned to the intervention group she also booked them in for the action and coping plan session. Therefore I did not know which group they were in until they came to the action and coping and plan setting session' (author correspondence)

Blinding of participants and personnel Unclear risk (performance bias)

All outcomes

Blinding of outcome assessment (detection Low risk bias)

The people delivering the exercises were blinded to group allocation. However, it was not feasible to blind the person involved in delivering the goal setting, and the action and coping planning, nor would it have been possible to blind the participants as they needed to be actively involved in the intervention

Outcome data were collected by research assistants blinded to group allocation

All outcomes

Incomplete outcome data (attrition bias) High risk

All outcomes

Outcome data collection adherence measures were not available for $7.4 \%$ of the study population. Self-efficacy scores were not available for $22.2 \%$ of the study population. Functional performance scores were not available for $29.7 \%$ of the study population. So the drop out rate ranged from less than $10 \%$ to more than $20 \%$ depending on the measure

Selective reporting (reporting bias) Unclear risk

Unable to find a protocol for this study published prior to it being conducted, so unable to compare the outcomes reported 
O’Brien 2013 (Continued)

with those planned to be measured at the outset

\begin{tabular}{lll}
\hline Other bias & Low risk & No evidence of other sources of bias \\
\hline
\end{tabular}

\section{Oestergaard 2012}

Methods

RCT

Setting: Orthopaedic spinal surgery unit within a University clinic, Denmark Funding: Danish Research Foundation of Occupational Therapy

Recruitment (patients): Patients were recruited from admissions to the spinal surgery unit between September 2003 and June 2004

Recruitment (healthcare providers): Not reported

Training/support: Not reported

Inclusion criteria (patients): Lumbar spinal fusion for degenerative disc disease

Exclusion criteria (patients): Less than 18 years; requiring an interpreter during treatment; senile dementia; hospitalised directly from a psychiatric institution; nursing home resident

Comorbidities: Not reported

Participants

Interventions
Patients: 87 participants (34.5\% male). Mean age 55 years (SD not reported; range 55 to 81 years). Ethnicity not reported

Principle health problems: Degenerative disc disease - 65.5\% disc degeneration/spondylosis; $34.5 \%$ with instability/spondylitis

Treatment currently receiving: Interprofessional inpatient rehabilitation following lumbar spinal fusion, including usual occupational therapy, i.e. 'instruction in using aids and appliances for bath and dressing activities and, when necessary, guidance in connection with kitchen activities' (p.116)

Description of healthcare providers: Four occupational therapists with experience in treating patients following lumbar spinal surgery

Study aim: To test the hypothesis that use of the Canadian Occupational Performance Measure (COPM) the guide problem identification, goal setting, and therapy planning in occupational therapy would result in identification of more problems with activities of daily living and better performance in activities of daily living after discharge from hospital for spinal surgery when compared to occupational therapy without use of the COPM

Intervention: ( $\mathrm{n}=40)$ COPM-guided occupational therapy following spinal surgery. The COPM used to identify and prioritise patient problems related to activities of daily living following surgery. Goals were set on the basis of this problem list, then 'the occupational therapist met with the physiotherapist and nursing staff to determine a joint course of action and to include the patient's goals in rehabilitation to occupational therapy, physiotherapy and nursing care' (p. 116)

Control: $(n=47)$ Occupational therapy without the use of COPM to guide problem identification, goal setting and treatment planning. Therapists in this group were still required to document problems with activities of daily living, rehabilitation goals and plans of action, but without use of the COPM

Delivery: All occupational therapy was delivered in the spinal surgery unit. Duration 
Oestergaard 2012 (Continued)

on admission was not reported

Fidelity: The experimental and the control group were treated by different occupational therapists, but adherence to treatment protocol for each group was not reported

Consumer involvement outside of the intervention: Not reported

$\begin{array}{ll}\text { Outcomes } & \text { Timing of outcomes: } 3 \text { months and } 3 \text { years after surgery } \\ \text { Number of problems with activities of daily living } & \text { Self-rated performance and satisfaction with performance on a list of } 18 \text { areas of activities } \\ \text { of daily living selected by the researchers } \\ \text { Dallas Pain Questionnaire } \\ \text { Length of hospitalisation } \\ \text { Duration of sick leave from work }\end{array}$

Notes

Risk of bias

Bias Authors' judgement

Random sequence generation (selection Low risk bias)

\section{Support for judgement}

'... patient were randomly assigned by the use of sealed envelopes...' (p.116)

'... patient were randomly assigned by the use of sealed envelopes...' (p.116)

The intervention required active involvement of the patient and healthcare professionals, so blinding not possible

The primary outcome was number of activity of daily living problem identified, which were generated by the patient and their therapist, both of whom were aware of group allocation. Two of the secondary 
Oestergaard 2012 (Continued)

outcomes were self-reported by the patient

Incomplete outcome data (attrition bias) Unclear risk

All outcomes

Selective reporting (reporting bias)

Unclear risk

Other bias

Unclear risk
'Follow-up rate was $91 \%$ after 3 months $69 \%$ after 3 years...' (p.117), so risk of attribution bias increased over time

Unable to find a protocol for this study published prior to it being conducted, so unable to compare the outcomes reported with those planned to be measured at the outset

'The experimental and the control group were treated by different occupational therapists' (p. 116). Therefore, low risk of crossgroup contamination. However the main outcome measure for the three month follow-up was manufactured for this study, and appeared to map onto the kinds of activities that would likely be generated from the COPM. The outcome measure at three years, the Dallas Pain Questionnaire (DPQ) appear to be added during the course of the study: 'In order to prevent reduced patient compliance, only the questionnaire composed for this study was used in the first three follow-ups. However, recent findings from a study with a similar patient category published in $2006 \mathrm{u}$ indicated that multiple questionnaires do not necessarily reduce patient compliance. Therefore, at the 3-year follow-up we chose to send both the questionnaire composed for this study and the DPQ to the patients for final follow-up' (p. 120) 
Recruitment (patients): Referred by nine neurosurgeons from the participating hospitals Recruitment (healthcare providers): Recruited from physiotherapists within the in participating hospitals

Inclusion criteria (patients): People who still have low back pain six weeks after firsttime disk surgery at only one level; 18-65 years; symptoms (e.g. pain) that restrict their normal daily living or work

Exclusion criteria (patients): Experiencing complications during surgery, as judged by a neurosurgeon based on pre-established criteria (e.g. loss of cerebrospinal fluid, nerve root lesions, or blood loss exceeding $600 \mathrm{~mL}$ ); patients with confirmed and relevant underlying diseases that influence activities of daily living (e.g. stenosis, malignancies); or if one of the treatments is contra-indicated (i.e., because of respiratory symptoms) Consideration of people with comorbidities: Not reported surgeon. Prior to randomisation, usual care for these patients consisted of the hospital physiotherapist providing training in low-back exercises and how to resume functions of normal daily living, and advice to resume normal activities as soon as possible. All patients are seen by the neurosurgeon again after six weeks

Description of healthcare providers: Physiotherapists trained in the intervention. No further demographic data provided with usual care in patients who still have low-back pain six weeks after first-time disk surgery

Intervention: ( $\mathrm{n}=52)$ Behaviour-graded activity based on goal setting. Patients identified two main complaints (specifically, activities that were important to them but could not be avoided). They were asked to perform these activities continuously until able to continue due to pain, after which they were guided to set personal goals for performance of these activities. A plan was set to increase the duration of performance these activity each day, by incremental amounts, from slightly less that the baseline performance level to the goal level within a three-month period. Activity quotas were to be performed exactly; not over-performed nor under-performed. Therapy included eighteen 30-minute sessions, plus daily home practice. All activities or exercises were recorded on a performance chart by the patient, with these charts being regularly discussed with physiotherapists during treatment sessions. Family members received education on the programme also. Activities were modified by the physiotherapist if goal achievement was proving difficult. Therapist engagement with patients was based on operant therapy principles

Control: ( $n=53$ ) Usual care based on the 'biomechanical model'. Therapy was based on pain-levels rather than behavioural goals, with decisions about therapy and exercises intensity being based on pain severity. Physiotherapy techniques including within 'usual care' included: exercises, education, and use of physical modalities. Acupuncture, osteopathic techniques and other alternative therapies were specifically excluded. Therapy consisted of 18 session of 30 mind duration over a three-month period, but the physio- 
Ostelo 2003 (Continued)

therapists for patients in this group were able to stop treatment when a patient no longer had symptoms and the treatment goals were being reached

Delivery: All goal setting and therapy delivered in 18 sessions over a three-month period Fidelity: Treatment sessions in both groups were selected at random, and recorded on audiotapes. These audio-recordings were reviewed by three blinded experts to determine if the treatments had been performed as prescribed

Consumer involvement outside of the intervention: Not reported

Outcomes

Timing of outcomes: Baseline, three months, six months, 12 months

Global Perceived Effect - a self-rated seven-point scale

Roland Disability Questionnaire

Tampa Scape for Kinesiophobia

Pain Catastrophising Scale

Pain Behaviour Scale

Pain intensity of the low back or leg, scored on a visual analogue scale

Severity of main complaint for the two frequently performed activities selected by the patient, with the severity of complaint scored on a visual analogue scale

Short Form-36

Costs, evaluated by cost diaries kept by patients, including additional therapies, drug use, visits to health care providers, out-of-pocket expenses, paid help, plus costs of loss of productivity because of low back pain-related absence from work

Range of flexion and extension of lumbar spine measured with a Cybex Electronic Digital Inclinometer-320

Number of relapses of low back pain and re-operations during the 12-month follow-up Number of withdrawals due to worsening symptoms

Goal setting characteristics

Comparison of interest: Collaborative, activity-oriented goal setting plus strategies to enhance goal pursuit versus usual care with no standardised or required approach to goal setting

Patient involvement in goal setting: Goals collaboratively set by the patient and healthcare professional, but with the therapist 'only as a coach in this goal-setting, because it is important that the goal is the patient's internal goal' (Ostello, 2000, p. 315)

Family involvement in goal setting: Not reported

Name of goal setting approach: No specifically named approach used

Development of a plan for goal pursuit: The goal setting approach involved a prescribed, incrementally step-wise plan to progress towards goal achievement

Written copy of goals provided to patients: Yes

Individual versus group-based goal discussion: Individual

Level of goal difficulty: Not reported

Goal areas of focus: Functional activities

Evaluation of patient goal commitment: Not reported

Goal reminders used: Goals were the focus of the regular therapy sessions

Monitoring of progress towards goals: All exercises and activities related to goals were documented by the patient on a performance chart and used to monitor progress towards goal, featuring in discussions with the physiotherapists at the regular therapy sessions

Notes

Power calculation: The study attempted to enrol 200 patients, with 100 patients per treatment arm. This sample size was deemed sufficient to detect a $20 \%$ difference in recovery rate (Global Perceived Effect) between the behavioural-graded activity program

Goal setting and strategies to enhance goal pursuit for adults with acquired disability participating in rehabilitation (Review)

Copyright @ 2015 The Cochrane Collaboration. Published by John Wiley \& Sons, Ltd. 
Ostelo 2003 (Continued)

and the usual care. 'We think that a $20 \%$ difference is clinically relevant; this difference is statistically significant at $\mathrm{cc}=.05$ with a power (1-beta) of $80 \%$. To obtain this study size we are co-operating with 4 hospitals and 75 physiotherapists' (Ostello 2000, p. 314)

\section{Risk of bias}

\begin{tabular}{|c|c|c|}
\hline Bias & Authors' judgement & Support for judgement \\
\hline $\begin{array}{l}\text { Random sequence generation (selection } \\
\text { bias) }\end{array}$ & Low risk & $\begin{array}{l}\text { Randomisation was '...based on computer- } \\
\text { generated randomization lists' (Ostelo et al. } \\
\text { 2003, p.1758) }\end{array}$ \\
\hline Allocation concealment (selection bias) & Low risk & $\begin{array}{l}\text { Allocation was concealed: 'By using } \\
\text { opaque, sealed, and coded randomization } \\
\text { envelopes' (Ostelo et al. 2003, p.175) }\end{array}$ \\
\hline $\begin{array}{l}\text { Blinding of participants and personnel } \\
\text { (performance bias) } \\
\text { All outcomes }\end{array}$ & High risk & $\begin{array}{l}\text { 'The patients are blinded to a certain extent } \\
\text { because they are unaware of the exact con- } \\
\text { tent of both treatments; these patients may } \\
\text { also be termed naive to the content of the } \\
\text { treatment not received' (p.314). However, } \\
\text { the treating physiotherapists could not be } \\
\text { blinded to group allocation and the inter- } \\
\text { vention itself required active involvement } \\
\text { of the patient and physiotherapists, so full } \\
\text { blinding was not possible }\end{array}$ \\
\hline
\end{tabular}

Blinding of outcome assessment (detection Unclear risk bias)

All outcomes
'... the research assistant who... performed the outcome assessments (M.R.K.) was blinded [to group allocation]' (Ostelo et al. 2003, p.1758). A number of the outcome measures were however based on self-reports by participants who were not blinded to group allocation
Incomplete outcome data (attrition bias) Unclear risk All outcomes
Of the 105 participants enrolled in the study, eight $(7.6 \%)$ withdrew before the post-treatment assessment, a cumulative total of $11(10.5 \%)$ had withdrawn before the six-month follow-up assessment, and a cumulative total of $12(11.4 \%)$ had withdrawn by the 12-month follow-up assessment. Intention-to-treat analysis followed in all cases. More specifically: 'On the post-treatment measurement, eight patients dropped out: one from the UC [usual care] group and seven from BGA [behaviour-graded activity]. The UC patient 
disappeared after two treatments without stating any reason and was therefore assigned the mean values of the UC group. The two BGA patients withdrew from the study due to aggravated symptoms; negative scores therefore substituted their values. One BGA patient showed an exacerbation of symptoms before treatment and another BGA patient suffered from rheumatic symptoms, a disease he had not mentioned before randomisation. These reasons were considered not to be related to the postoperative treatment; therefore, they were assigned mean values. One BGA patient reported to be completely pain-free after two treatment sessions and was no longer motivated to continue the study, and one patient stepped out when resuming his professional occupation full time (without residual signs or symptoms) due to lack of time and motivation. One BGA patient withdrew because of personal circumstances and had actually recovered after five treatment sessions. The values of these three patients were substituted by positive values. After 6 months follow-up, 2 more BGA-patients dropped out: one underwent an operation for an intestinal disorder, and the other patient dropped out without obvious reasons and did not react to several voice mail requests. Both patients were assigned mean values. Another UC patient dropped out because of aggravated symptoms; negative values were used for substitution. After 12 months of follow-up, another BGA patient dropped out due to aggravated symptoms; negative values were used for substitution' (Ostelo, 2003, p.1760-1761)

Selective reporting (reporting bias) Low risk

No evidence of selective reporting. Reported outcomes match those proposed in a protocol published in Ostello et al (2000) prior to undertaking the study

Other bias

Low risk

No evidence of other sources of bias

Goal setting and strategies to enhance goal pursuit for adults with acquired disability participating in rehabilitation (Review) 
Methods

Participants
Cluster-RCT. Participants clustered by the General Practitioner (family physician) they were registered with

Setting: Community, New Zealand

Funding: New Zealand Health Research Council Disability Research Placement Programme Grant and a PhD scholarship

Recruitment (patients): Recruited from new referrals of community-dwelling older adults for homecare

Recruitment (healthcare providers): Not reported

Inclusion criteria (patients): Community-dwelling people 65 years and over were new referrals for homecare support

Exclusion criteria (patients): cognitive impairment impacting on ability to adhere to intervention and give consent (based on Abbreviated Mental Test Score $<7 / 10$ ), or if referred for assessment for residential care admission, and carer support was only for short-term services

Consideration of people with comorbidities: Not reported

Patients: 205 participants (33.7\% male). Mean age 79.08 (intervention group), 76.90 (control group) (SD 6.93 intervention group, 7.61 control group). 77.6\% Caucasian; $22.4 \%$ other ethnic groups. $62.9 \%$ living alone; $37.1 \%$ living with others

Principle health problems: Age-related disability

Treatment currently receiving: Home care support

Description of healthcare providers: Not reported
Study aim: To determine whether provision of restorative home support to older people based on the TARGET approach to goal setting would result in improvements in healthrelated quality of life and ability to undertake activities of daily living when compared with a group receiving standard homecare

Intervention: ( $\mathrm{n}=108$ ) Restorative home support (based on use of the TARGET approach to goal setting)

TARGET stands for 'Towards Achieving Realistic Goal in Elders Tool'. This group had an initial assessment which involved identifying a goal for the homecare episode, and subsequent rehabilitation aims. These were then passed from the assessment agency to the homecare organisation where a support plan was developed. The plan included tasks to be undertaken by the support workers. Assessment staff delivering the TARGET tool and all homecare co-ordinators attended a standardised two and half day training programme before the start of the study

Control: ( $\mathrm{n}=97)$ Standard needs assessment based on professional opinion of the assessor. Participants then referred to a homecare organisation. Assessment staff in this group did not receive the TARGET training

Delivery: All goal setting and home care support delivered over a six-month period Fidelity: Not reported

Consumer involvement outside of the intervention: Not reported
Timing of outcomes: Baseline and 6 months follow-up

Short form-36 - Physical Component

Short form-36 - Mental Component

Number of client reviews undertaken by homecare co-ordinators

Mortality 
Parsons 2012 (Continued)

Goal setting characteristics

Comparison of interest: Collaborative goal setting versus usual care with no standardised or required approach to goal setting

Patient involvement in goal setting: Goals collaboratively set by the patient and healthcare professional

Family involvement in goal setting: None reported

Name of goal setting approach: TARGET

Development of a plan for goal pursuit: Goal used to develop a support care plan, including instructions for tasks to be undertaken

Written copy of goals provided to patients: Not reported

Individual versus group-based goal discussion: Individual

Level of goal difficulty: Not reported

Goal areas of focus: Not reported

Evaluation of patient goal commitment: Not reported

Goal reminders used: Not reported

Monitoring of progress towards goals: Not reported

Notes

Power calculation: Not reported

Risk of bias

\begin{tabular}{l|l|l}
\hline Bias & Authors' judgement & Support for judgement \\
\hline $\begin{array}{l}\text { Random sequence generation (selection } \\
\text { bias) }\end{array}$ & Low risk & $\begin{array}{l}\text { Participants were clustered by general prac- } \\
\text { titioner, and clusters were allocated to the } \\
\text { intervention or control conditions based } \\
\text { on 'the use of a randomly generated nu- } \\
\text { meric list' (p.26). Microsoft Excel was used } \\
\text { to generate the random sequence (author } \\
\text { communication) }\end{array}$ \\
\hline
\end{tabular}

Allocation concealment (selection bias) Low risk

The research staff involved in recruiting participants to the study and allocating them to general practitioners were blinded to the allocation of clusters to intervention or control conditions (author communication)

Blinding of participants and personnel High risk (performance bias)

All outcomes

The intervention required active involvement of the patient and healthcare professionals, so blinding not possible

Blinding of outcome assessment (detection Unclear risk bias)

All outcomes

'Assessments were undertaken by experienced researchers blinded to group allocation.' (p.25). However outcome assessments was based on self-report by participants who were not blinded to group allocation

Goal setting and strategies to enhance goal pursuit for adults with acquired disability participating in rehabilitation (Review) 
Parsons 2012 (Continued)

\begin{tabular}{|l|l|l|l}
\hline $\begin{array}{l}\text { Incomplete outcome data (attrition bias) } \\
\text { All outcomes }\end{array}$ & Low risk & $\begin{array}{l}\text { Of the } 205 \text { participants enrolled in the } \\
\text { study, four died and four withdrew (a total } \\
\text { of } 3.9 \% \text { attrition) prior to the six month } \\
\text { data collection. 'Evaluations were under- } \\
\text { taken on the 'Intention to Treat' principle' } \\
\text { (p.26) }\end{array}$
\end{tabular}

Selective reporting (reporting bias) Low risk

No evidence of selective reporting. Reported outcomes match those proposed in a protocol published in the Australian New Zealand Clinical Trials Registry prior to undertaking the study

\begin{tabular}{|c|c|c|}
\hline Other bias & Low risk & No evidence of other sources of bias \\
\hline
\end{tabular}

Richardson 2007

\begin{tabular}{ll}
\hline Methods & RCT \\
Setting: University of Michigan Hospital, USA \\
Funding: Michigan Diabetes Research and Training Center, the Center for Health Com- \\
munications Research, and the lead author's career development award and Physician \\
Faculty Scholars Program award from the Robert Wood Johnson Foundation \\
Recruitment (patients): Recruited via public advertisements and referrals from physicians \\
Recruitment (healthcare providers): Not reported \\
Inclusion criteria (patients): Inclusion: Type 2 diabetes; 18 years of age, regular email \\
users with access to the Internet using Window 2000 or XP; self-reportedly exercising \\
less than 150 minutes per week of moderate physical activity at baseline; interested in \\
starting a walking programme; medical clearance to start a walking programme from \\
physician; English speaking \\
Exclusion criteria (patients): Having had used a pedometer in the past 30 days, or were \\
pregnant \\
Consideration of people with comorbidities: Not reported \\
\hline
\end{tabular}

Participants

Patients: 35 participants enrolled. 30 participants completed the study (33.3\% male). Mean age 52 (total step group); 53 (bout step group) (SD 12, total step group; 9 bout step group). Age range 38 to 71 years. $76.7 \%$ White, $13.3 \%$ Black, $10 \%$ other ethnic groups

Principle health problems: Type 2 diabetes

Treatment currently receiving: Not reported

Description of healthcare providers: Health professional only involved in set up of the programme. The therapy was automated, delivered by an Internet-based computer programme

Interventions

Study aim: 'The purpose of this study was to compare two different goal setting strategies in a pedometer-based walking program for people with type 2 diabetes; one employing lifestyle goals (LG) for overall steps and the other employing structured goals (SG) that emphasize greater activity intensity' (Background section, para. 5)

Participants in both groups in this study were asked to wear a pedometer every day,

Goal setting and strategies to enhance goal pursuit for adults with acquired disability participating in rehabilitation (Review) 

step-count data at any time. The pedometer connected to the participants' personal computers and uploaded information about their daily, hour-by-hour, step-count data to an online server, which the participants were encouraged to access at least once a week. The website also provided motivational messages and tips about managing diabetes. The website prescribed weekly exercise goals for the participants to achieve, automatically calculating these goals from the step-count data upload by the participant, and providing graphs to display their performance in relation to the goals. The nature of goals set were the subject of the interventions in this study

Intervention (LG): ( $n=19)$ Lifestyle goals. Participants were automatically calculated a new goal each week to increase their total accumulated steps. Participants were encouraged to focus on increasing their amount of time spent during bouts of moderate intensity exercise, but step-count data during bouts of moderate intensity exercise were not a factor that contributed to the weekly goals

Intervention (SG): ( $n=16)$ Structured goals. Participants were automatically calculated a new goal each week to increase their step-count data when undertaking bouts of exercise at moderate intensity and duration (a minimum of ten minutes walking at an intensity of at least 60 steps per minute). All graphs and data display on the website reported stepcount data related to these bouts of moderate intensity exercise, so could report zero steps when participants did not meet the criteria for minimum duration and intensity of exercise

Delivery: The goal setting and step-count data were delivered over a six-week period

Fidelity: Not reported. However the automated nature of the intervention reduced bias usually associated with healthcare professionals delivering therapy during a study

Consumer involvement outside of the intervention: Not reported

Outcomes

Goal setting characteristics
Timing of outcomes: At the end of the six-week study period

Steps taken during bouts of walking that last for at least ten minutes at an intensity of at least 60 steps per minute

Patient satisfaction

Patient adherence

Comparison of interest: Setting of a specific, prescribed goal regarding total exercise regardless of intensity versus setting of a specific, prescribed goal regarding total exercise of a moderate to high intensity only

Patient involvement in goal setting: None. Goals prescribed according to group allocation Family involvement in goal setting: None reported

Name of goal setting approach: No specifically named approach used

Development of a plan for goal pursuit: Patients provided general motivational message via the website into which they uploaded their study data

Written copy of goals provided to patients: Yes

Individual versus group-based goal discussion: Individual

Level of goal difficulty: Rather than goal difficulty, this study compared two different types of goals, i.e. ones focusing on total step-count at any intensity versus ones focusing only on step-count related to exercise at moderately high intensity or greater

Goal areas of focus: Walking, measured by step-count data

Evaluation of patient goal commitment: Not reported

Goal reminders used: Reminders about goals provided every time the participants upload

their weekly data onto the study website 
Richardson 2007 (Continued)

Monitoring of progress towards goals: Feedback on progress towards goal was provide graphically on the study website

Notes Power calculation: Not reported. However the study was reported as a pilot RCT

Risk of bias

\begin{tabular}{|c|c|c|}
\hline Bias & Authors' judgement & Support for judgement \\
\hline $\begin{array}{l}\text { Random sequence generation (selection } \\
\text { bias) }\end{array}$ & Unclear risk & $\begin{array}{l}\text { Participants'...were randomized with equal } \\
\text { probability into one of the two intervention } \\
\text { groups' (Methods section, para.6) }\end{array}$ \\
\hline Allocation concealment (selection bias) & Unclear risk & No information provided \\
\hline $\begin{array}{l}\text { Blinding of participants and personnel } \\
\text { (performance bias) } \\
\text { All outcomes }\end{array}$ & Low risk & $\begin{array}{l}\text { Delivery of the intervention was automated } \\
\text { by the study website, so no healthcare pro- } \\
\text { fessionals were involved }\end{array}$ \\
\hline $\begin{array}{l}\text { Blinding of outcome assessment (detection } \\
\text { bias) } \\
\text { All outcomes }\end{array}$ & Low risk & $\begin{array}{l}\text { All outcome data were automatically up- } \\
\text { loaded from the pedometers used by par- } \\
\text { ticipants in the study }\end{array}$ \\
\hline $\begin{array}{l}\text { Incomplete outcome data (attrition bias) } \\
\text { All outcomes }\end{array}$ & Unclear risk & $\begin{array}{l}\text { Five of the } 35(14.3 \%) \text { participants initial } \\
\text { enrolled were no longer in the study or did } \\
\text { not provide data at the end of the study }\end{array}$ \\
\hline Selective reporting (reporting bias) & Low risk & $\begin{array}{l}\text { No evidence of selective reporting. Re- } \\
\text { ported outcomes match those proposed in } \\
\text { a protocol published in ClinicalTrials.gov, } \\
\text { a service of the US National Institutes of } \\
\text { Health }\end{array}$ \\
\hline Other bias & Low risk & No evidence of other sources of bias \\
\hline
\end{tabular}


Methods
RCT

Setting: Community, Midwest USA

Funding: Supported by American Heart Association - Midwest Affiliate

Recruitment (patients): Participants recruited from two visiting nursing association agencies (i.e. community nursing agencies), who screened daily admissions for the primary diagnosis of heart failure

Recruitment (healthcare providers): Nurses were employees of the visiting nursing agencies

Inclusion criteria (patients): over 18 years of age; receiving home healthcare for primary diagnosis of heart failure; English-speakers

Exclusion criteria (patients): Not further criteria reported

Consideration of people with comorbidities: Not reported
Patients: 88 participants (56\% male). Mean age 75 (SD 12). Range 33 to 90 years. Ethnicity not reported

Duration of heart failure: 32 with $<1$ year, 30 with $1-5$ years, 26 with $>5$ years

Principle health problems: Heart failure $(36.4 \%<1$ year's duration; 34.1\% 1-5 year's duration; $29.5 \%>5$ year's duration)

Treatment currently receiving: Home nursing support

Description of healthcare providers: Nursing from the agencies heart failure teams. Further demographic characteristics not provided versus placebo instruction on patient understanding of heart failure and self-efficacy for managing heart failure in a sample of people receiving home-based nursing care

Intervention (Supportive education): $(\mathrm{n}=28)$ In addition to usual nursing care, this group received teaching in self-care management of heart failure provided at weekly meetings of one hour or less over an eight-week period. Topics included: learning to live with heart disease, assessing patient and caregiver prior learning, reviewing support systems, developing plans for needs related to self-management

Intervention (Mutual goal setting): $(\mathrm{n}=27)$ In addition to usual nursing care, the group received collaborative goal setting with nurses, based on King's nursing theory of goal attainment (King 1981), plus planning the means to achieve goals, provided at weekly meetings of one hour or less over an eight-week period. Goals were collaboratively developed between patient and nurse; prioritised by the patients in order of importance; re-evaluated at a specific time; measurable

Control: $(n=33)$ In addition to usual nursing care, this group received instruction about health promotion, not on topic specific to heart failure, (e.g. skin care, injury prevention, insomnia, dental care, foot care, and food-borne illnesses), provided at weekly meetings of one hour or less over an eight-week period

Delivery: All goal setting and interventions delivered over an eight-week period

Fidelity: Not evaluated. However 'Each nursing approach provider was educated for only one approach and administered only that approach to ensure that each approach was accurate and not influenced by knowledge of another approach' (p.504)

Consumer involvement outside of the intervention: Not reported confidence in understanding management of heart failure

Self-efficacy to Manage Disease in General 
Scott 2004 (Continued)

Mental Health Inventory-5 (a subscale of the Short Form-36) (at 3 and 6 months only)

The cardiac version of the Quality of Life Index (at 3 and 6 months only)

Goal setting characteristics

Comparison of interest: Collaborative goal setting plus strategies to enhance goal pursuit versus patient training/education versus attention control with no goal setting

Patient involvement in goal setting: Goals collaboratively set by the patient and healthcare professional

Family involvement in goal setting: None reported

Name of goal setting approach: Based on Imogene King's (1981) theory of goal attainment (King 1981)

Development of a plan for goal pursuit: The goal setting approached used included identification of strategy for how to achieve the goals

Written copy of goals provided to patients: Not reported

Individual versus group-based goal discussion: Individual

Level of goal difficulty: Not reported

Goal areas of focus: Not reported

Evaluation of patient goal commitment: Not reported

Goal reminders used: Not reported

Monitoring of progress towards goals: Not reported

Notes

Power calculation: In Setter Kline et al (2007): 'An attempt was made to obtain 31 subjects for each group on the basis of power analysis before data collection' (p.504). In Scott et al (2004): 'For studies using three repeated measures (entry into the study, 3 and 6 months), with an alpha .05 , a power of .80 , and a moderate effect size, 30 participants were needed for each intervention group' (p.250). In Ranta (2000): 'The goal was to include 31 subjects in the control and 31 subjects in the intervention group, to achieve a power of $80 \%$. With a power equal to .80 , there is a $20 \%$ risk of committing a Type II error" (p.25). However no information was provided about what outcome measure (or variance) this power calculation was based on, or what was deemed a moderate effect size

Risk of bias

Bias Authors' judgement

Random sequence generation (selection Low risk bias)

\section{Support for judgement}

'As subjects were enrolled in the study, the principal investigator made random assignments to 1 of 3 nursing approaches... ' (Scott et al., 2004, p.504). The sequence for random assignments was established before any participants were recruited by way of a random numbers table (author communication)

Participants were recruited by research assistants who were blind to the randomisation sequence. Once recruited, a participant's details were then pass onto the primary researcher, who then assignment that 
participant to whichever treatment group was next on the random sequence list (author communication)

Blinding of participants and personnel High risk (performance bias)

All outcomes

Blinding of outcome assessment (detection Unclear risk

bias)

All outcomes

All outcomes

High risk

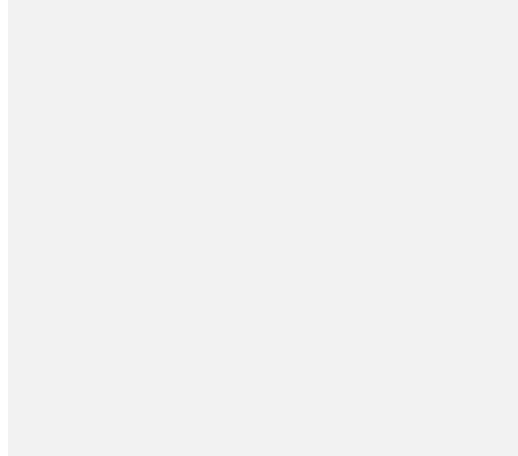

Selective reporting (reporting bias)

Unclear risk
The intervention required active involvement of the patient and healthcare professionals, so blinding not possible

Outcome assessors were blinded to group allocation (author communication). Most outcome measures were however based on self-report by participants who were not blinded to group allocation

Of the 88 participants who enrolled, 22 (25\%) were no longer in the study at 6 months, and $32(36.4 \%)$ were no longer in the study at 12 months. These participants were excluded from the analysis. In the mutual goal setting group, 24 enrolled in the study, 17 remained at 3 months, 15 remained at 6 and 12 months. In the supportive education group, 27 enrolled in the study, and 17 remained at 3,6 , and 12 months. In the control group, 31 enrolled in the study, 27 remained at 3 month, and 24 remained at 6 and 12 months (author communication)

Data collected at for multiple outcomes at multiple point (3, 6, 9, 12 months). The data are no longer available, which makes evaluation of reporting bias difficult. Unable to find a protocol for this study published prior to it being conducted, so unable to compare the outcomes reported with those planned to be measured at the outset

\begin{tabular}{lll} 
Other bias & Low risk & No evidence of other sources of bias \\
\hline
\end{tabular}


Methods
RCT

Setting: A hospital in Leicester, UK

Funding: Trent Regional Research Scheme

Recruitment (patients): Recruited from a hospital-based pulmonary rehabilitation assessment clinic

Recruitment (healthcare providers): Not reported

Inclusion criteria (patients): Adults with stable Chronic Obstructive Pulmonary Disease, with no hospital admission or exacerbations for four weeks preceding the assessment Exclusion criteria (patients): No further criteria reported

Consideration of people with comorbidities: Not reported
Participants
Patients: 180 participants (61.7\% male). Mean age 68 (SD 9). Ethnicity not reported Principle health problems: Chronic obstructive pulmonary disease (Mean FEV1 0.95L, SD 0.4L; Mean FEV1/FVC ratio: 0.51 (SD 0.15); 12.2\% on long-term oxygen therapy; $19.4 \%$ current smokers

Treatment currently receiving: Not additional therapy reported except for the pulmonary rehabilitation provided as part of the study

Description of healthcare providers: Not stated simpler general exercise program without individualised goal setting. A second aim was to identify whether pulmonary rehabilitation improved domestic activity and increased the level of functional independence in the home

All participants received twice weekly, hospital-based pulmonary rehabilitation for seven weeks. Sessions involved one hour of exercise and one hour of education. All participants were also asked to complete daily training walks at home

Intervention: ( $\mathrm{n}=90)$ Individualised goal-directed exercise programme. Prior to beginning the pulmonary rehabilitation classes, participants completed a Canadian Occupational Performance Measure (COPM) assessment. They were then prescribed a set of ten exercises specifically designed to address the daily activity goals identified during the COPM assessment. These exercises were undertaken during the hospital-based and home based exercise sessions. Pulmonary rehabilitation staff regularly reinforced the link between the individualised exercises and the participants goals during the hospital based sessions

Control: $(\mathrm{n}=90)$ As for the intervention group (including completion of a COPM assessment), but instead of setting individualised goals, these participants followed a standardised exercise programme. The same ten exercises were given to all participants to practice in both the hospital and home exercise sessions

Delivery: All exercises were completed over a seven-week period

Fidelity: Not reported

Consumer involvement outside of the intervention: Not reported weeks)

Physical activity measured by an ambulatory activity monitor worn for two consecutive days, for 12 hours each day

Canadian Occupational Performance (COPM) Performance and Satisfaction scores

Incremental shuttle walk test

Chronic Respiratory Questionnaire 
Sewell 2005 (Continued)

Goal setting characteristics
Comparison of interest: Collaborative goal setting plus exercise prescription to enhance goal pursuit versus no goal setting and standardised exercise prescription

Patient involvement in goal setting: Patients guided by therapist to identify 'the five most important daily activities they would like or need to do but found difficult to complete because of their respiratory illness' (p.1195)

Family involvement in goal setting: None reported

Name of goal setting approach: Based on used of the COPM for goal selection

Development of a plan for goal pursuit: Exercises for therapy were prescribed based on the individualised goals set

Written copy of goals provided to patients: Not reported

Individual versus group-based goal discussion: Individual and group-based discussions Level of goal difficulty: Not reported

Goal areas of focus: Activities of daily living

Evaluation of patient goal commitment: Not reported

Goal reminders used: The pulmonary rehabilitation staff 'gave verbal reinforcement to the subjects during the classes that these exercises replicated the activities that they had each identified as goals'

Monitoring of progress towards goals: Not reported as measured by an ambulatory activity monitor. Previous pilot data estimated that a mean increase of 2000 counts (over a cumulative period of $24 \mathrm{~h}$ ) would need to be detected between the groups. It was calculated that 64 patients in each group would need to be recruited to attain a $5 \%$ significance level with $80 \%$ power' (p.1195)

\section{Risk of bias}

\section{Bias}

Authors' judgement

Support for judgement

Random sequence generation (selection Low risk bias)
'Patients were randomly assigned to one of two treatment groups' (p.1195). 'Cards with the treatment allocation were placed in identical sealed envelopes and shuffled by a team member not involved with the study. The envelopes were then shuffled again by a further team member not related to the study. The envelopes were placed in a locked drawer and accessed by the pulmonary rehabilitation team once the participant had provided informed written consent' (author communication)

Allocation concealment (selection bias) Low risk
'Randomization was completed using sequentially numbered, sealed envelopes and were opened by the [pulmonary rehabilitation] staff. The lead investigator was blinded to the subject randomization until 
Sewell 2005 (Continued)

all of the interventions had been allocated and completed' (p.1195)

Blinding of participants and personnel High risk (performance bias)

All outcomes

Blinding of outcome assessment (detection Unclear risk bias)

All outcomes
The intervention required active involvement of the patient and healthcare professionals, so blinding not possible

All staff involved in outcome assessment were blinded to group allocation. Outcome assessments occurred in a different part of the hospital to where the interventions were provided (author communication). Some outcome measures were however based on self-report by participants who were not blinded to group allocation

Incomplete outcome data (attrition bias) High risk All outcomes

o risk

Unable to find a protocol for this study published prior to it being conducted, so unable to compare the outcomes reported with those planned to be measured at the outset

\section{Other bias}

Low risk 
Methods
Cluster-RCT. Participants clustered by mental health service

Setting: Ten community mental health centres, USA

Funding: Janssen, a division of Ortho-McNeil-Janssen Pharmaceuticals, Inc

Recruitment (patients): The study was conducted between May 2009 and March 2010, but the process for patient recruitment was not reported

Recruitment (healthcare providers): The community mental health centres were selected on the basis of their participation in the National Council on Community Behavioral Healthcare's Enhanced Access and Retention Quality Improvement Initiative. Healthcare providers were recruited from these centres

Training/support: Providers in the experimental group received training via videoconferencing in person-centred care planning, plus monthly coaching and monitoring in their delivery of this intervention

Inclusion criteria (patients): 18 years or older; one or more psychiatric hospitalizations or two or more psychiatric emergency room visits in the past year; DSM-IV axis I diagnosis, meeting at least two functional criteria of severe mental illness

Exclusion criteria (patients): No additional exclusion criteria were reported

Comorbidities: Not reported
Participants

Interventions
Patients: 367 participants. $25.6 \%<40$ years; $58.6 \% 40-60$ years; $15.8 \%>60$ years. Gender not reported. Ethnicity not reported

Principle health problems: Mental health disorders - $41.7 \%$ schizophrenia; $24.0 \%$ bipolar disorder; $23.4 \%$ depression; $10.9 \%$ other

Treatment currently receiving: Community mental health services (details of these services not reported)

Description of healthcare providers: 84 providers from the experimental community mental health centres, including frontline staff and supervisors, were recruited and trained for the study. No details were reported on the numbers or type of providers recruited in the control centres

Study aim: To test the hypothesis that person-centred care planning (via collaborative goal setting) would result in better treatment engagement and medication adherence for people with mental health condition than usual practice without goal setting

At the start of the study, mental health services in both the intervention and control groups were involved in the introduction of a new centralised scheduling and management system for clients who failed to attend appointments. All services in both groups were required to have a treatment plan which outlined each client's goals and objectives based on their individually assessed needs

Intervention: ( $\mathrm{n}=177$ ) Person-centred goal setting and care planning, involving identification of participant life goals, translation of these life goals into action steps (developing service plans to integrate life goals), and maintenance of a focus on these life goals during therapeutic sessions. Follow-up appointments included discussion of missed appointments and how to avoid missing them, collaborative discussion and development of assessment, planning, and evaluation documentation to identify and integrate participant life goals with more traditional mental health goals. All documentation was completed during face-to-face sessions with the clients

Control: $(n=190)$ Goals for the clients in the control group were developed without client involvement. The healthcare providers in these services receiving no additional training in person-centred planning and collaborative documentation

Delivery: Duration or frequency of treatment sessions for the intervention and control 
group was not reported

Fidelity: Providers for the experimental group received monthly coaching and monitoring of their delivery of the intervention

Consumer involvement outside of the intervention: None reported

\begin{tabular}{ll} 
Outcomes & $\begin{array}{l}\text { Timing of outcomes: Data collected monthly over an 11-month period } \\
\text { Medication adherence } \\
\text { Attendance at scheduled appointments }\end{array}$ \\
\hline Goal setting characteristics & $\begin{array}{l}\text { Comparison of interest: Goal setting plus strategies to enhance goal pursuit versus no } \\
\text { goal setting } \\
\text { Patient involvement in goal setting: All goals collaboratively set by the clients and their } \\
\text { healthcare workers, based on the clients' life goals }\end{array}$ \\
Family involvement in goal setting: None reported \\
$\begin{array}{l}\text { Name of goal setting approach: No specifically named approach used } \\
\text { Development of a plan for goal pursuit: Collaboratively developed by the clients and } \\
\text { their healthcare workers } \\
\text { Written copy of goals provided to patients: Not reported } \\
\text { Level of goal difficulty: Not reported } \\
\text { Goal areas of focus: Not reported } \\
\text { Evaluation of patient goal commitment: Not reported } \\
\text { Goal reminders used: Reminders regarding goals was provided at each therapy session } \\
\text { Monitoring of progress towards goals: Progress towards goals was collaboratively dis- } \\
\text { cussed at each therapy session }\end{array}$ \\
\hline
\end{tabular}

Notes

Risk of bias

\begin{tabular}{l|l} 
Bias Authors' judgement $\quad$ Support for judgement
\end{tabular}

Random sequence generation (selection Low risk bias)

Allocation concealment (selection bias) Low risk

Blinding of participants and personnel High risk (performance bias)

All outcomes
Five of the ten community mental health services were selected for the structured goal setting intervention by picking their names, at random, out of a container (author communication)

Selection of services names from the container was concealed. Participants were already clients of the mental health services at the time that allocation of services to study groups occurred (author communication)

The intervention required active involvement of the patient and healthcare professionals, so blinding not possible 
Stanhope 2013 (Continued)

\begin{tabular}{|c|c|c|}
\hline $\begin{array}{l}\text { Blinding of outcome assessment (detection } \\
\text { bias) } \\
\text { All outcomes }\end{array}$ & High risk & $\begin{array}{l}\text { Data collection was managed by healthcare } \\
\text { providers, so this also was not blinded }\end{array}$ \\
\hline $\begin{array}{l}\text { Incomplete outcome data (attrition bias) } \\
\text { All outcomes }\end{array}$ & Unclear risk & $\begin{array}{l}\text { Of the } 367 \text { participants enrolled in the } \\
\text { study, outcome data were not available on } \\
109 \text { of them by study end. The loss of par- } \\
\text { ticipant was unequal across the groups: } 19 / \\
117 \text { from the experimental group and } 90 / \\
190 \text { from the control group (RR } 2.92,95 \% \\
\text { CI } 1.88 \text { to } 4.52 \text { ). However, as adherence } \\
\text { was the main outcome of interest in this } \\
\text { study, this difference in attrition rates could } \\
\text { be considered an outcome as opposed to a } \\
\text { methodological limitation of the study }\end{array}$ \\
\hline Selective reporting (reporting bias) & Unclear risk & $\begin{array}{l}\text { Unable to find a protocol for this study } \\
\text { published prior to it being conducted, so } \\
\text { unable to compare the outcomes reported } \\
\text { with those planned to be measured at the } \\
\text { outset }\end{array}$ \\
\hline Other bias & Unclear risk & $\begin{array}{l}\text { The experimental group providers received } \\
\text { additional training, coaching and mentor- } \\
\text { ing (on an ongoing basis) that the control } \\
\text { group providers did not receive }\end{array}$ \\
\hline
\end{tabular}

\section{Taylor 2012}

Methods
Cluster-RCT. Participants clustered by rehabilitation unit

Setting: Four inpatient rehabilitation hospital units, New Zealand

Funding: Health Research Council of New Zealand Feasibility Study Grant

Recruitment (patients): Participants were recruited from four inpatient rehabilitation wards

Recruitment (healthcare providers): Not reported

Inclusion criteria (patients): Admitted to an inpatient rehabilitation service with a primary diagnosis of stroke

Exclusion criteria (patients): Unable to reliably engage in a Canadian Occupational Performance Measure (COPM) assessment or outcome assessments for the following reasons - delirium or dementia (as determined by Mini-Mental State Examination score < 24), dysphasia, significant visual or auditory impairment, non-English speaking Consideration of people with comorbidities: Data on the number of comorbid diseases was collected, using the self-administered 18-item Functional Comorbidity Index

Participants
Patients: 41 participants (63.4\% male). Mean age 58.5 (intervention group); 58.5 years (control group) (SD 15.9 intervention group; 16.6 control group)

Principle health problems: Cerebrovascular disorder (87.8\% first ever stroke; $70.7 \%$ left side stroke). Functional Comorbidity Index: 2.9 (SD 2.1) and 2.8 (SD 1.2) for the 
Taylor 2012 (Continued)

intervention control groups respectively

Treatment currently receiving: Inpatient stroke rehabilitation

Description of healthcare providers: An occupational therapist, trained in the COPM, not otherwise involved in rehabilitation services on the ward completed the COPM assessment and goal setting. All other rehabilitation services were provided by the usual interdisciplinary teams working on the ward involved in the study

Interventions

Study aim: The aim of this study was to test the feasibility of a cluster-RCT design for development of a fully powered study. Cluster randomisation was being investigated as a means of managing problems with cross-group contamination in a standard RCT involving goal setting in an inpatient environment. The aim of a full study would be to test the effectiveness of an enhanced, person-centred approach to goal setting, based on use of the COPM, versus usual inpatient rehabilitation to improvement outcomes for people with stroke

Intervention: ( $\mathrm{n}=18$ ) COPM-based goal setting. Use of COPM to set high-level personcentred goals on top of usual inpatient goal setting and rehabilitation for stroke. This involved: 1) an Occupational Therapist completing a COPM assessment within one week of admission, which was used to elicit specific, concrete, short-term goals and higher order long-term goals, 2) active engagement of the patient in goal setting, and 3) feedback and communication of goals set with the multidisciplinary team (documenting these in the front of the patient's clinical file)

Control: ( $\mathrm{n}=23)$ The control group received identical baseline and follow-up assessments except that the COPM was not administered. 'Participating rehabilitation services used goal-setting as part of their usual care but this process was not typically structured or organized to the same degree as the COPM and was often framed in terms of discipline-specific goals by individual therapists (e.g. a physiotherapist may set a goal of 'sliding board transfer with one assist'; and may or may not be congruent with the participants' goals). The point of difference between this and the intervention condition was the structured elicitation of participants' difficulties and priorities, which was then communicated clearly to the clinical team'(p.330)

Delivery: COPM based goal setting and communication of these goals occurred over the first week of assessment. Inpatient rehabilitation then proceeded as normal

Fidelity: Not evaluated. The lack of monitoring of treatment fidelity was raised as an issue of particular concern in the discussion of the study

Consumer involvement outside of the intervention: Not reported

Outcomes

Timing of outcomes: At baseline, discharge, and 12 weeks follow-up

Schedule for Evaluation of Individual Quality of Life

Short Form-36

Functional Independence Measure

Patient Perception of Rehabilitation

Goal setting characteristics

Comparison of interest: Enhanced patient involvement in collaborative goal setting versus usual care

Patient involvement in goal setting: Goals collaboratively set by the patient and healthcare professional

Family involvement in goal setting: None reported

Name of goal setting approach: Based on used of the COPM for goal selection

Development of a plan for goal pursuit: Not included as part of the study intervention.

Goal setting and strategies to enhance goal pursuit for adults with acquired disability participating in rehabilitation (Review) 
Taylor 2012 (Continued)

Left up to the clinical team implementing the rehabilitation programme

Written copy of goals provided to patients: Not reported

Individual versus group-based goal discussion: Individual

Level of goal difficulty: Not reported

Goal areas of focus: Activity limitation and participation restrictions

Evaluation of patient goal commitment: Not reported

Goal reminders used: Not included as part of the study intervention

Monitoring of progress towards goals: Not reported

Notes

Power calculation: "The sample size for the pilot aimed for 15 participants per cluster and four clusters, a total of 60 participants. The sample size was chosen in order to have two clusters per randomized treatment and the number of participants per cluster was based on the number of degrees of freedom needed within each cluster to have reasonable precision to estimate a variance, where the suggested residual degrees of freedom should be between 10 and 15.9. The study was not designed to have statistical power in order to detect a difference between the two treatments as neither the size of the ICC nor the likely size of the minimum clinically important difference for the SEIQOL-DW were known" (p.329)

\section{Risk of bias}

\begin{tabular}{l|l} 
Bias Authors' judgement & Support for judgement
\end{tabular}

Random sequence generation (selection Low risk bias)

Allocation concealment (selection bias) Low risk

Blinding of participants and personnel High risk (performance bias)

All outcomes

Blinding of outcome assessment (detection Unclear risk

bias)

All outcomes

Incomplete outcome data (attrition bias) Low risk All outcomes
Participants were clustered by rehabilitation unit. Rehabiltation units were randomised to the intervention or control condition using an online randomisation service to generate the random sequence

A researcher blinded to the allocation of the clinical service to study groups was responsible for recruiting and enrolling participants into the study

The intervention required active involvement of the patient and healthcare professionals, so blinding not possible

Outcome assessors were blinded to group allocation. Some outcome measures were however based on self-report by participants who were not blinded to group allocation

Complete data were gathered for $92.7 \%$ of participants, with incomplete data for one participant in the intervention group and two participants in the control group 
Taylor 2012 (Continued)

\begin{tabular}{l|l|l}
\hline Selective reporting (reporting bias) & Low risk & $\begin{array}{l}\text { No evidence of selective reporting. Re- } \\
\text { ported outcomes match those proposed in } \\
\text { a protocol published in the Australian New } \\
\text { Zealand Clinical Trials Registry prior to } \\
\text { undertaking the study }\end{array}$ \\
\hline Other bias & Low risk & No evidence of other sources of bias \\
\hline
\end{tabular}

\section{Webb 1994}

$\begin{array}{ll}\text { Methods } & \text { RCT } \\ \text { Setting: Residential and day centre services for people with brain injury and in Indi- } \\ \text { anapolis, USA } \\ \text { Funding: Indiana University - Purdue University } \\ \text { Recruitment (patients): Long terms residents or day clients of a Brain Injury Unit } \\ \text { Recruitment (healthcare providers): Not reported } \\ \text { Inclusion criteria (patients): over } 16 \text { years; oriented to person, place, time, and memory } \\ \text { for events which occurred before and after the injury - scoring } 80 \text { or above on the } \\ \text { Galveston Orientation and Amnesia Test; intact awareness of disability and having a } \\ \text { desire to change - evaluated using the Change Assessment Questionnaire, with a higher } \\ \text { score on the 'Contemplation' and 'Action' stages of the questionnaire compared to scores } \\ \text { on the 'Precontemplation' stage } \\ \text { Exclusion criteria (patients): No further criteria reported } \\ \text { Consideration of people with comorbidities: Not reported }\end{array}$

Participants

Patients: 16 participants (87.5\% male). Mean age 27 (SD 5). 100\% Caucasian. 81.3\% residential clients, $12.7 \%$ day clients

Other demographic characteristics: 13 were residents, 3 were day clients

Average years since onset of disability: 8.7 (SD 5.5)

Years of education: 12.1 (SD 1.9)

Average days in coma: 88.9 (SD 76.9)

Principle health problems: traumatic brain injury. Average length of coma (days) 88.9

(SD 76.9). Average years since onset of brain injury 8.7 (SD 5.5)

Treatment currently receiving: Residential or day hospital rehabilitation for traumatic brain injury

Description of healthcare providers: Nine therapists were involved (55.5\% with a Master's degree; $44.5 \%$ with a Bachelor's degree), including one psychologist, one recreational therapist, one occupational therapist, two speech therapists, and four other therapists with degrees in psychology-related fields

Interventions

Study aim: '...to test the effect of direct involvement in goal setting on specific rehabilitation outcomes for persons with [traumatic brain injury]. The specific hypotheses of the present study were as follows: 1. Experimental participants (HI) will show greater achievement of goals from pre-testing to post-testing than control participants (LI). 2. Experimental participants (HI) will show greater achievement of goals from pre-testing to the two-month follow-up than control participants (LI).' (p. 180)

Intervention aim:

Intervention: $(\mathrm{n}=8)$ High involvement $(\mathrm{HI})$ in goal setting. Participants met with a ther-

Goal setting and strategies to enhance goal pursuit for adults with acquired disability participating in rehabilitation (Review) 
apist for approximately one hour per week for eight weeks. Therapy consisted firstly of formal orientation and training in goal setting, discussion of the importance of personal goal setting, and opportunities for participants to ask questions about goal setting. Individual participant goal areas were identified based on information from rehabilitation team notes, and written on small wooden blocks, which the participants then physically arranged in order of importance. Therapists then assisted participants to formulate a specific, behavioural goal-based on their first priority goal area. Therapists documented the level of difficulty of this goal, and convert this goal into a Goal Attainment Scaling (GAS) scale for the next eight weeks. Therapy during the eight weekly sessions then incorporated discussion and review of goal progress, using worksheets and a goal diary to facilitate participants monitoring and rating their own progress using the GAS scale Control: $(n=8)$ Low involvement (LI) in goal setting. Participants met with a therapist for approximately one hour per week for eight weeks. Therapy consisted firstly of formal orientation to goal setting, but no discussion of the importance of personal goal setting and not encouragement for participants to ask questions about goal setting. Individual participant goal areas were identified based on information from rehabilitation team notes, and written on paper for the participants to prioritise. Therapists then assisted participants to formulate a specific, behavioural goal-based on their first priority goal area. Therapists documented the level of difficulty of this goal, and convert this goal into a GAS scale for the next eight weeks. Therapy during the eight weekly sessions occurred without the use of goal assessment techniques, worksheets, or goal follow-up diary

Delivery: Intervention and goal discussions occurred over an eight-week period

Fidelity: Not reported

Consumer involvement outside of the intervention: Not reported

Outcomes

Goal setting characteristics
Timing of outcomes: Baseline, one week after treatment, and two months after treatment GAS. Goal attainment was rated by the treating therapist AND an 'independent rater'. 'Therapists developed behaviourally-anchored scale points for each goal in collaboration with the participant. The [treating] therapists also made periodic GAS rating, as did the independent raters. Ratings by independent raters were highly correlated with primary therapists' ratings $(\mathrm{r}=.99, \mathrm{P}<.001)$ and thus, the former's ratings were used in the final analysis of results as the dependent measure' (p. 183)

Comparison of interest: Collaborative goal setting plus strategies to enhance goal pursuit versus usual care with less patient involvement in goal setting and no additional strategies to enhance goal pursuit

Patient involvement in goal setting: Goals collaboratively set by the patient and healthcare professional

Family involvement in goal setting: None reported

Name of goal setting approach: GAS

Development of a plan for goal pursuit: Not reported

Written copy of goals provided to patients: Yes

Individual versus group-based goal discussion: Individual

Level of goal difficulty: Evaluated by the therapist, but not apparently restricted during the goal setting process

Goal areas of focus: Not reported

Evaluation of patient goal commitment: Not reported

Goal reminders used: Goal discussed at the weekly therapy meetings

Monitoring of progress towards goals: Patient self-monitored their perceived goal progress 
and rated their own goal attainment levels on structured worksheets which were discussed in the weekly therapy meetings

\begin{tabular}{ll}
\hline Notes & Power calculation: Not reported \\
\hline
\end{tabular}

\section{Risk of bias}

\begin{tabular}{|c|c|c|}
\hline Bias & Authors' judgement & Support for judgement \\
\hline $\begin{array}{l}\text { Random sequence generation (selection } \\
\text { bias) }\end{array}$ & Unclear risk & $\begin{array}{l}\text { '...participants were randomly assigned...' } \\
\text { (p. 181) Insufficient information provided } \\
\text { to determine whether adequate sequence } \\
\text { generation }\end{array}$ \\
\hline Allocation concealment (selection bias) & Unclear risk & No information provided \\
\hline
\end{tabular}

Blinding of participants and personnel High risk (performance bias)

All outcomes

Blinding of outcome assessment (detection Unclear risk bias)

All outcomes

Incomplete outcome data (attrition bias) High risk All outcomes

Selective reporting (reporting bias) Unclear risk
The intervention required active involvement of the patient and healthcare professionals, so blinding not possible

A therapist, 'who was a staff member unaware of the hypothesis of the study' (p. 183), completed the outcome assessment, but it is not stated whether this person was aware of the content of intervention that the participants in each group received

Of the 16 participants who enrolled in the study, five $(31.3 \%)$ were lost to follow-up and not included in the analysis

Unable to find a protocol for this study published prior to it being conducted, so unable to compare the outcomes reported with those planned to be measured at the outset

Other bias High risk
The therapists who were involved in delivering the intervention were also involved in constructing the individualised scales for GAS rating, with this occurring after randomisation, although the scoring of outcomes on these scale was undertaken by an independent outcome assessment blinded to the study hypothesis 
Methods

Participants
Cluster-RCT. Participants clustered by the case manager they were assigned to

Setting: Three urban community mental health services, USA

Funding: West Family Foundation and the Segal Family Foundation

Recruitment (patients): Not reported

Recruitment (healthcare providers): Not reported

Inclusion criteria (patients): Not reported, but all participating clients had a diagnosis of a mental health disorders and had to be scheduled for a regularly occurring six-month care plan

Exclusion criteria (patients): Not reported

Consideration of people with comorbidities: People with current substance abuse or dependency were included ( $20 \%$ of total population enrolled)

Patients: 80 participants (66.3\% male). Mean age 47 (intervention group); 46 (control group) (SD 9 intervention group; 11 control group). 33.8\% White; 60.0\% African American; 6.2\% Latino

Principle health problems: Mental health disorders. 60.0\% schizophrenia or schizoaffective disorders; $18.8 \%$ bipolar disorder; $15 \%$ major depressive disorder; $1.2 \%$ posttraumatic stress disorder; $2.5 \%$ other mental health disorder

Treatment currently receiving: Community-based mental health support

Description of healthcare providers: 20 case managers (26.3\% male). Mean age 47 (intervention group); 31 (control group) (SD 12 intervention group; 7 control group). 42. 1\% White; 50.6\% African American; 5.3\% Asian

Mean years working in mental health field 12 (intervention group); 5 (control group) (SD 10 intervention group; 4 control group). 52.6\% of case managers had a Master's degree

Interventions
Study aim: This study examined whether use of an electronic decision support system to create a shared decision-making plan, structured around collaborative goal setting, led to greater client and case manager satisfaction with the care planning process than the usual agency procedures for creating a care plan. Also investigated was whether clients are more engaged in the process of care planning, as measured by recall of the goals included in the care plan three days after the planning session

Intervention: $(\mathrm{n}=40)$ Electronic decision support system (for goal setting and treatment planning) involving three-steps: 1) Clients indicated their top priorities and ideas for services at a touchscreen-enabled computer kiosk, 2) this information was sent to the case managers, who then complete a similar process, and 3) the two perspectives were then merged electronically and presented graphically in a shared decision-making session with the two participants. The clients met with their case managers a minimum of three months before their regularly scheduled six-month care plans were due in order to keep this process separate from their usual six-month care planning. These clients eventually completed their six-month care plans as scheduled (as required for billing purposes), but were able to use information from the electronic decision support system to guide this six-month plan

Control: $(n=40)$ Met with case managers at the time that their six-month care plans were due. Care plan discussions were audio-recorded and completed following the case managers usual method. Usual care included 'use of a behavioural health electronic medical record designed to aid with billing and to theoretically help case managers create recovery-oriented care plans. There appeared to be significant heterogeneity in how care plans were completed, as is the case in real-world case management' (p.55) 
Outcomes

Timing of outcomes: Recall of goals was reported two to four days after the care planning meeting. The timing of other outcome data collection was not clear

A case manager satisfaction questionnaire (comprised of six 5-point Likert type scale questions)

A client satisfaction questionnaire (comprised of seven 5-point Likert type scale questions)

Proportion of care plan goals correctly recalled by the client

Goal setting characteristics

Comparison of interest: Collaborative goal setting versus usual care with no standardised or required approach to goal setting

Patient involvement in goal setting: Goals collaboratively set by the patient and healthcare professional

Family involvement in goal setting: None reported

Name of goal setting approach: No specifically named approach used

Development of a plan for goal pursuit: Not reported

Written copy of goals provided to patients: Not reported

Individual versus group-based goal discussion: Individual

Level of goal difficulty: Not reported

Goal areas of focus: Not reported

Evaluation of patient goal commitment: Not reported

Goal reminders used: Not reported

Monitoring of progress towards goals: Not reported

Power calculation: Not reported

Risk of bias

Bias

Random sequence generation (selection Unclear risk bias)

\section{Authors' judgement}

\section{Support for judgement}

'Case managers from three clinics were randomly assigned to the intervention group of treatment as usual. Clients were assigned to the same group to which their case manager was assigned' (p.55). Insufficient information provided to determine whether adequate sequence generation

No information provided

Allocation concealment (selection bias) Unclear risk

The intervention required active involvement of the patient and case managers, so blinding was not possible All outcomes

Blinding of participants and personnel High risk

Blinding of outcome assessment (detection High risk bias)

All outcomes

All data based on self-report by the patients and case managers, so blinding was not possible 


\begin{tabular}{|c|c|c|}
\hline $\begin{array}{l}\text { Incomplete outcome data (attrition bias) } \\
\text { All outcomes }\end{array}$ & Unclear risk & $\begin{array}{l}\text { Data from the case managers was avail- } \\
\text { able for } 78 \text { of the } 80(97.5 \%) \text { intervention } \\
\text { episodes. Sixty-nine }(86 \%) \text { of the } 80 \text { clients } \\
\text { were successfully contacted two to four days } \\
\text { after participation in the case planning ses- } \\
\text { sion, but with more successful contacted } \\
\text { in the control group than the intervention } \\
\text { group }(90 \% \text { versus } 83 \%)\end{array}$ \\
\hline Selective reporting (reporting bias) & Unclear risk & $\begin{array}{l}\text { Unable to find a protocol for this study } \\
\text { published prior to it being conducted, so } \\
\text { unable to compare the outcomes reported } \\
\text { with those planned to be measured at the } \\
\text { outset }\end{array}$ \\
\hline Other bias & Unclear risk & $\begin{array}{l}\text { Participants in both groups usually met } \\
\text { with their case managers every six months } \\
\text { to complete a six-month care plan. The in- } \\
\text { tervention group in this study met with } \\
\text { their case manager an additional time three } \\
\text { months before their next six-month care } \\
\text { plan was due. The results regarding satisfac- } \\
\text { tion with service process could have arisen } \\
\text { from the more regular client-case manager } \\
\text { contact rather than anything specifically to } \\
\text { do with the approach to goal planning that } \\
\text { was used }\end{array}$ \\
\hline
\end{tabular}

COPM = Canadian Occupational Performance Measure; GAP = Goal setting and Planning skills programme; GAS = Goal Attainment Scaling; GMT = Goal Management Training; ICF = International Classification of Functioning, Disability and Health; OBM = operant behaviour management; $\mathrm{SD}=$ standard deviation; WBI = Work Behavioral Inventory

\section{Characteristics of excluded studies [ordered by study ID]}

\begin{tabular}{ll}
\hline Study & Reason for exclusion \\
\hline Adachi 1989 & $\begin{array}{l}\text { Does not involve a population of people with disability acquired in adulthood. Involves a weight loss } \\
\text { intervention for people who are overweight }\end{array}$ \\
\hline Adair 2013 & $\begin{array}{l}\text { The type of goals set did not meet the review definition of a 'rehabilitation goal'. Many instead described } \\
\text { medical interventions to be carried out (e.g. 'Measurement of urinary albumin within } 2 \text { y'; 'Pneumonia } \\
\text { vaccination'; 'ACEI or ARB prescription if LVEF 0.40' (p. 177) }\end{array}$
\end{tabular}

Goal setting and strategies to enhance goal pursuit for adults with acquired disability participating in rehabilitation (Review) 
(Continued)

\begin{tabular}{l|l} 
Alfonso 2011 & $\begin{array}{l}\text { Unable to separate the effects of goal setting or strategies to enhance goal pursuit from another interven- } \\
\text { tion, specifically mindfulness meditation }\end{array}$ \\
\hline Alicea-Planas 2013 & $\begin{array}{l}\text { Insufficient information that the study population had a disability acquired in adulthood. Involves people } \\
\text { in a general community population receiving primary care nursing for conditions including infectious } \\
\text { diseases and gastric reflux }\end{array}$ \\
\hline Bailey 1988 & Not a RCT or quasi-RCT \\
\hline Blackberry 2013 & $\begin{array}{l}\text { Unable to separate the effects of goal setting or strategies to enhance goal pursuit from the broader } \\
\text { intervention of telephone coaching }\end{array}$
\end{tabular}

Bonde 2005

Unable to separate the effects of goal setting or strategies to enhance goal pursuit from other interventions, including counselling and work skills training

Chan 2012

Does not involve a population of people with disability acquired in adulthood. Involves a population of non-disabled people aiming to increase general physical activity

Christiansen 2010

Unable to separate the effects of goal setting or strategies to enhance goal pursuit from other psychological interventions, including cognitive behavioural therapy

Clare 2013

Unable to separate the effects of goal setting or strategies to enhance goal pursuit from the broader intervention of cognitive rehabilitation

Conrin 1985

Unable to separate the effects of goal setting or strategies to enhance goal pursuit from other psychological interventions

Curtin 1997

Does not involve a population of people with disability acquired in adulthood. Involves a population of people classified as 'heavy drinkers' but not necessarily with a diagnosable alcohol addiction

Curtin 2001

Does not involve a population of people with disability acquired in adulthood. Involves a population of people classified as 'heavy drinkers' but not necessarily with a diagnosable alcohol addiction

Drebing 2005

Does not involved a controlled trial investigating a goal setting intervention; the focus instead is on the clinical effects of reward-based performance

Duarte 2012

Not a RCT or quasi-RCT

Estabrooks 2005

Not a RCT or quasi-RCT

Evans-Hudnall 2012

Unable to separate the effects of goal setting or strategies to enhance goal pursuit from the broader intervention of training in self-management for stroke

Faett 2012

Unable to separate the effects of goal setting or strategies to enhance goal pursuit from the broader intervention of training in self-management

Goal setting and strategies to enhance goal pursuit for adults with acquired disability participating in rehabilitation (Review) 
(Continued) Gorton 2009
of the diabetes management intervention

Graven $2012 \quad$ Unable to separate the effects of goal setting or strategies to enhance goal pursuit from the broader intervention involving extensive additional community therapy and support

Greene $2000 \quad$ Unable to separate the effects of goal setting or strategies to enhance goal pursuit from the broader intervention involving early supported discharge for people with stroke (author communication)

Hansen $2011 \quad$ Unable to separate the effects of goal setting or strategies to enhance goal pursuit from the other aspects of the diabetes management intervention

Huang 2010

Insufficient information that the study population had a disability acquired in adulthood. Involves population of people with type 2 diabetes with unclear age of onset

Huisman 2010

Unable to separate out the effects of goal setting or strategies to enhance goal pursuit from other aspects of invention, including peer-group meetings, motivational interviewing, and being given a pedometer

Jeffery 2003

No all participants were people who had a disability acquired in adulthood. Includes non-disabled participants who are overweight but not clinically obese

Johnson 2009

Unable to separate out the effects of goal setting or strategies to enhance goal pursuit from other aspects of the behaviour change invention

Katz 2011

Unable to separate out the effects of goal setting or strategies to enhance goal pursuit as the study compares

GMT with another type of therapy to address problems with executive functioning

Kelley $2004 \quad$ Not a RCT investigating the effects of goal setting

Kerr 2012

Does not specifically involve a population of people with disability acquired in adulthood. Involves nondisabled older adults. Unable to separate the effects of goal setting or strategies to enhance goal pursuit from the broader intervention involving additional physical activity

Kerse 2008

Unable to separate out the effects of goal setting or strategies to enhance goal pursuit from the other aspects of the individualised activities of daily living activity programme

Lenze 2012

This study investigated strategies for changing health professional behaviour rather than investigating the specific effects of goal setting or strategies to enhance goal pursuit

Levine 2007

Does not involve a population of people with disability acquired in adulthood. Involves non-disabled older adults

Levine 2011

Not a RCT or quasi-RCT

Liang 1984

Unable to separate out the effects of goal setting or strategies to enhance goal pursuit from the other aspects of the outreach rehabilitation program

Goal setting and strategies to enhance goal pursuit for adults with acquired disability participating in rehabilitation (Review) 
(Continued)

\begin{tabular}{ll}
\hline Linton 1984 & Not a RCT or quasi-RCT \\
\hline Lozano 2010 & $\begin{array}{l}\text { Does not involve a population of people with disability acquired in adulthood. Involves a population of } \\
\text { people classified as 'heavy drinkers' but not necessarily with a diagnosable alcohol addiction }\end{array}$ \\
\hline Mate-Kole 1999 & $\begin{array}{l}\text { Does not involve a population of people with disability acquired in adulthood, rather people with } \\
\text { congenital conditions are involved }\end{array}$ \\
\hline Mitka 2013 & Not a RCT or quasi-RCT \\
\hline
\end{tabular}

Motl 2012 Not a report from a controlled trial, but a report on a secondary analysis of observational data from just the intervention arm of a controlled trial

Naik 2011

Unable to separate out the effects of goal setting or strategies to enhance goal pursuit from the other aspects of the diabetes management programme

Novakovic-Agopian 2011 Allocation to treatment groups not randomised or pseudo-randomised

O’Connor $2006 \quad$ Not a RCT or quasi-RCT

O'Connor 2008 Insufficient information that the study population had a disability acquired in adulthood. Involves a population of people with intermittent allergic rhinitis with unclear age of onset

Pandit $2010 \quad$ Not a RCT or quasi-RCT

Pankow 2000

Goal attainment was used as an outcome measure, and was not the focus of the intervention under investigation

Petry 2006

Does not investigate the effects of goal setting or strategies to enhance goal pursuit, where the terms 'goal' matches the definition of 'rehabilitation goals' used for the purposes of this review

Rodgers 2013

Does not investigate the effects of goal setting or strategies to enhance goal pursuit, where the terms 'goal' matches the definition of 'rehabilitation goals' used for the purposes of this review

Rokke $1999 \quad$ Not a RCT or quasi-RCT

Smith $2011 \quad$ Insufficient information that the study population had a disability acquired in adulthood. Involves a population of people with allergic rhinitis with unclear age of onset

Sperduto 1986

Does not involve a population of people with disability acquired in adulthood. Includes non-disabled participants who are overweight but not necessarily clinically obese

Spikman 2010

Unable to separate the effects of goal setting or strategies to enhance goal pursuit from the broader intervention of cognitive rehabilitation

St John 1973

Not a RCT or quasi-RCT

Goal setting and strategies to enhance goal pursuit for adults with acquired disability participating in rehabilitation (Review) 
(Continued)

\begin{tabular}{|c|c|}
\hline Stenstrom 1994 & $\begin{array}{l}\text { Compared a goal setting intervention to a pain attention intervention, thus unable to separate the effects } \\
\text { of goal setting or strategies to enhance goal pursuit when interpreting the study results }\end{array}$ \\
\hline Stubberud 2013 & $\begin{array}{l}\text { Does not involve a population of people with disability acquired in adulthood. Involves adults with spina } \\
\text { bifida, a congential neurological condition }\end{array}$ \\
\hline Stuifbergen 2003 & $\begin{array}{l}\text { Unable to separate out the effects of goal setting or strategies to enhance goal pursuit from other aspects } \\
\text { of the behaviour change intervention }\end{array}$ \\
\hline Stuifbergen 2010 & $\begin{array}{l}\text { Unable to separate out the effects of goal setting or strategies to enhance goal pursuit from other aspects } \\
\text { of the behaviour change intervention }\end{array}$ \\
\hline van Hooren 2007 & $\begin{array}{l}\text { Does not involve a population of people with disability acquired in adulthood, rather older people with } \\
\text { subclinical, self-reported memory problems were involved }\end{array}$ \\
\hline Weg 2009 & $\begin{array}{l}\text { Insufficient information that the study population had a disability acquired in adulthood. Involves a } \\
\text { convenience sample of patients from a general medical ward including 'pediatrics, geriatrics, postoperative } \\
\text { general and orthopedic surgical, observation status, acute care status, and long-term care' (p.20) }\end{array}$ \\
\hline Wilson 2010 & $\begin{array}{l}\text { Does not involve a population of people with disability acquired in adulthood. Includes people with } \\
\text { asthma with unclear age of onset }\end{array}$ \\
\hline Wood 2012 & $\begin{array}{l}\text { Did not involve investigation of a goal setting intervention over a period of time longer than a single } \\
\text { clinical session }\end{array}$ \\
\hline Wressle 2002 & Not a RCT or quasi-RCT \\
\hline Zegman 1983 & $\begin{array}{l}\text { Insufficient information that the study population had a disability acquired in adulthood. Includes non- } \\
\text { disabled participants who are overweight but not necessarily clinically obese }\end{array}$ \\
\hline
\end{tabular}

GMT = Goal Management Training; RCT $=r$ andomised controlled trial

Characteristics of ongoing studies [ordered by study ID]

\section{ACTRN12609000433202}

\begin{tabular}{l|l}
\hline Trial name or title & Goals and self regulation skills in brain injury rehabilitation: a Randomised Clinical Trial \\
\hline Methods & RCT \\
\hline Participants & $\begin{array}{l}\text { Adult with traumatic brain injury with a history of post-traumatic amnesia greater than } 1 \text { hour, moderate } \\
\text { disability on the Extended Glasgow Outcome Scale, receiving compensation from a national accident com- } \\
\text { pensation organisation for } 12 \text { weeks (indicating resultant disability), and are } 6 \text { to } 18 \text { months post-injury }\end{array}$
\end{tabular}

Goal setting and strategies to enhance goal pursuit for adults with acquired disability participating in rehabilitation (Review) 


\section{ACTRN12609000433202 (Continued)}

\begin{tabular}{ll}
\hline Interventions & $\begin{array}{l}\text { Intervention 1: A novel intervention aiming to improve goal related activity and self regulation skills (goals- } \\
\text { SR). The intervention uses components of both Identity Oriented Goal Training and Goal Management } \\
\text { Training. Delivered weekly for } 8 \text { weeks during intervention sessions lasting approximately one hour }\end{array}$ \\
& $\begin{array}{l}\text { Intervention 2: Goal Attainment Scaling (GAS) measurement tool, administered weekly over an 8-week } \\
\text { period during intervention sessions lasting approximately one hour } \\
\text { Control: Usual practice (i.e. no additional intervention from the researchers), with participants continuing } \\
\text { with their planned rehabilitation process, details of which will be collected at the end of the study period }\end{array}$ \\
\hline Outcomes & $\begin{array}{l}\text { Primary: Goal related skills as measured by Self-Regulatory Skills Interview; self and carer report on Neu- } \\
\text { robehavioural Functioning Inventory } \\
\text { Secondary: Progress towards achieving meaningful goals in participant's life as measured by the Schedule for } \\
\text { Evaluation of Individual Quality of Life - Direct Weighting; social integration, carer burden; and well being } \\
\text { as assessed by questionnaires }\end{array}$ \\
\hline Starting date & Data collection completed; authors still writing final results up for publication \\
\hline Contact information & Kathryn McPherson: kathryn.mcpherson@aut.ac.nz \\
\hline Notes & \\
\hline
\end{tabular}

\section{Arends 2013}

Trial name or title A goal management intervention for polyarthritis patients

Methods $\quad$ Randomised controlled trial with blocked stratified randomisation per site in random block sizes of two and four to ensure that both intervention and control conditions are equally distributed in each of four participating hospitals

Participants People who are age of 18 years or over, have a diagnosis of polyarthritis, and score of four or higher on the depression subscale of the Hospital Anxiety and Depression Scale

Interventions Intervention: Six group-based meetings with eight to ten participants per group. Meeting focus on goal management strategies, specifically: goal maintenance, goal adjustment, goal disengagement, and goal reengagement

Control: Wait-list control

Outcomes $\quad$ Primary outcome: The depression subscale of the Hospital Anxiety and Depression Scale

Secondary outcomes: The anxiety subscale of the Hospital Anxety and Depression Scale; the positive subscale of the Positive and Negative Affect Schedule; the Purpose In Life Scale; the Impact on Participation and Autonomy questionnaire; a visual analogue scale for pain; a visual analogue scale for fatigue; physical functioning subscale of the Short Form-36; the Tenacious Goal Pursuit scale; the Flexible Goal Adjustment scale; the Goal Adjustment Scale; Goal Management Strategy Vignettes; the versatility subscale of the Coping Flexibility Questionnaire; and the Arthritis Self-efficacy Scale

Starting date Not stated

Contact information Roos Arends: R.Y.Arends@utwente.nl 
Arends 2013 (Continued)

Notes

Bertens 2013

Trial name or title A randomized controlled trial on errorless learning in goal management training

Methods $\quad$ RCT using a computer generated block randomisation procedure without stratification

Participants Adults, 18-70 years, with non-progressive acquired brain injury, at least 3 months after injury, in outpatient rehabilitation, having executive deficits as established by a neuropsychological examination, and living independently at home

Interventions Group 1: Goal management training with errorless learning

Group 2: Goal management training with trial and error learning

Both conditions will be run as eight 1-hour individual sessions, delivered twice a week

Outcomes Primary outcome measure: Activity of daily living task performance

Secondary outcome measures: GAS; executive function tests (Brixton spatial anticipation test, Category fluency test, Go/No-go task subtest Test for Attentional Performance, Letter fluency test, Letter number sequencing, subtest WAIS III, Modified six elements test, Zoo map test, subtest Behavioural Assessment of the Dysexecutive Syndrome); memory (Rivermead behavioural memory test-third edition); attention and concentration (Alertness task, subtest Test for Attentional Performance); estimation IQ (National adult reading test - Dutch version); subjective cognitive functioning (Cognitive failures questionnaire); dysexecutive behaviour (Dysexecutive questionnaire); self-reported executive functioning (Executive function index); observed executive functioning (Executive observation scale); quality of life (Short Form-36)

Starting date

June 2012

Contact information Dirk Bertens: d.bertens@donders.ru.nl

Notes

\section{Dawson 2013}

\begin{tabular}{ll} 
Trial name or title & $\begin{array}{l}\text { Managing executive dysfunction following acquired brain injury and stroke using an ecologically valid reha- } \\
\text { bilitation approach: a study protocol for a randomized, controlled trial }\end{array}$ \\
\hline Methods & RCT using blocks randomisation via a random numbers table \\
\hline Participants & Survivors of acquired brain injury or stroke recruited via community agencies \\
\hline Interventions & $\begin{array}{l}\text { Intervention: The Cognitive Orientation to daily Occupatonal Performance. This intervention involves teach- } \\
\text { ing patients to set goals, plan for goal achievement, implement their plan, and evaluate their goal achieve- } \\
\text { ment (i.e. "Goal, Plan, Do, Check"). This is delivered in treatment sessions with an occupational therapist, } \\
\text { involving up to } 15 \text { hours of contact therapy time } \\
\text { Control: Up to } 15 \text { hours of conventional, publicly-funded community-base occupational therapy }\end{array}$
\end{tabular}

Goal setting and strategies to enhance goal pursuit for adults with acquired disability participating in rehabilitation (Review) 


\section{Dawson 2013 (Continued)}

\begin{tabular}{l|l}
\hline Outcomes & $\begin{array}{l}\text { Primary outcome: Canadian Occupational Performance Measure } \\
\text { Secondary outcomes: Performance Quality Rating Scale; Behavioural Rating Inventory of Executive Function } \\
\text { - Adult; Mayo-Portland Adaptability Inventory Participation Index; Instrumental Acivities of Daily Living } \\
\text { Profile }\end{array}$ \\
\hline Starting date & June 2013 \\
\hline Contact information & Deirdre Dawson: ddawson@research.baycrest.org \\
\hline Notes & \\
\hline
\end{tabular}

\section{Gracey 2012}

Trial name or title The effectiveness of brief goal management training (GMT) and SMS text alerts on psychosocial functioning following brain injury: the Assisted Intention Monitoring (AIM) Trial

Methods Randomised controlled trial, partial cross-over design

Participants Adults with non-progressive brain injury, acquired in adulthood, more than 1 year post-injury, with everyday organisational and memory problems reported by themselves, their carer, or a clinician. Participants need to have and be able to use a mobile phone

Interventions Intervention: Two to three hours of GMT, followed by a 3-week period where participants are sent text messages to their phone reminding them to "STOP!" (Stop, Think, Organise, Plan)

Control: Two hours of general information about brain injury, some 'brain training' games, and neutral text messages

Outcomes Mean proportion of all daily intentions achieved for each intervention phase; Profile of Mood States

Starting date Data collection completed; authors still writing final results up for publication

Contact information Fergus Gracey: fg290@medschl.cam.ac.uk

Notes

\section{Mansfield 2013}

Trial name or title Using wireless technology in clinical practice: does feedback of daily walking activity improve walking outcomes of individuals receiving rehabilitation post-stroke? Study protocol for a randomized controlled trial

\begin{tabular}{ll}
\hline Methods & RCT using a blocked stratified randomisation \\
\hline Participants & Adult with subacute stroke attending inpatient rehabilitation \\
\hline Interventions & $\begin{array}{l}\text { Intervention: Goal setting for physiotherapy goals related to mobility; plus daily feedback on progress towards } \\
\text { goals using accelerometery to evaluate current mobility performance in relation to set goals. All intervention } \\
\text { delivered within the context of a 6-16 week inpatient and outpatient rehabilitation programme for stroke }\end{array}$ \\
\hline
\end{tabular}


Mansfield 2013 (Continued)

Control: Goal setting and 6-16 weeks of inpatient and outpatient rehabilitation as per the Intervention group protocol, but without the daily accelerometer-base feedback on progress towards goals

\begin{tabular}{ll} 
Outcomes & $\begin{array}{l}\text { Primary outcomes: mobility activity - specifically total walking duration, total number of steps taken and } \\
\text { total distance walked, duration of continuous walking, frequency and duration of longer walk bouts }(>5 \\
\text { minutes continuously), frequency and duration of high intensity walking (i.e. walking at a speed of } \geq 85 \% \\
\text { of maximum walking velocity) } \\
\text { Secondary outcomes: Stroke Self-Efficacy Questionnaire; Community Integration Questionaire, goal attain- } \\
\text { ment }\end{array}$ \\
\hline Starting date & June 2013 \\
\hline Contact information & Avril Mansfield: avril.mansfield@uhn.ca \\
\hline Notes &
\end{tabular}

\section{Novakovic-Agopian 2012}

\begin{tabular}{ll}
\hline Trial name or title & Goal-Oriented Attention Regulation Training in Veterans with Chronic TBI \\
\hline Methods & RCT \\
\hline Participants & $\begin{array}{l}\text { Veterans with chronic traumatic brain injury (at least 6 months after injury) and mild-moderate executive } \\
\text { dysfunction }\end{array}$ \\
\hline Interventions & $\begin{array}{l}\text { Intervention: Five weeks of either GOALS training consisting of goal-oriented attentional self-regulation } \\
\text { training designed to target and improve such deficits via training applied to individually-defined goals } \\
\text { Control: Five weeks of brain health education as an active control matched in therapy time and intensity }\end{array}$ \\
\hline Outcomes & $\begin{array}{l}\text { Neuropsychological tests; assessments of functional performance in complex 'real-life' settings; self-reported } \\
\text { measures of emotional regulation and daily functioning }\end{array}$ \\
\hline Starting date & 2012 \\
\hline Contact information & Anthony Chen: anthony_chen@post.harvard.edu \\
\hline Notes & \\
\hline
\end{tabular}

\section{Reiser 2012}

\begin{tabular}{ll}
\hline Trial name or title & Effectiveness of an online aftercare program \\
\hline Methods & RCT \\
\hline
\end{tabular}

Participants

People with psychosomatic disorders receiving inpatient rehabilitation 
Reiser 2012 (Continued)

Interventions
home, allocating two weeks to focus on the achievement of each goal. These goals are then entered into a
web-based portal and after discharge patients are invited (on a bi-weekly basis) to provide feedback on their
goal attainment by logging on to the online portal
Control: No aftercare

Outcomes

Questionnaires assessing reported symptom severity (exact questionnaires not stated)

Starting date Not stated

Contact information Not stated. Attempts to contact these authors by emailing to organisation affiliated with this research have been unsuccessful

Notes 
DATA AND ANALYSES

Comparison 1. Goal setting (with or without strategies to enhance goal pursuit) versus no goal setting

\begin{tabular}{|c|c|c|c|c|}
\hline Outcome or subgroup title & $\begin{array}{l}\text { No. of } \\
\text { studies }\end{array}$ & $\begin{array}{c}\text { No. of } \\
\text { participants }\end{array}$ & Statistical method & Effect size \\
\hline $\begin{array}{l}1 \text { Health related quality of life or } \\
\text { self-reported emotional status }\end{array}$ & 8 & 446 & Std. Mean Difference (IV, Random, 95\% CI) & $0.53[0.17,0.88]$ \\
\hline 2 Activity - ability & 4 & 223 & Std. Mean Difference (IV, Random, 95\% CI) & $0.04[-0.22,0.31]$ \\
\hline 3 Engagement in rehabilitation & 9 & 369 & Std. Mean Difference (IV, Random, 95\% CI) & $0.30[-0.07,0.66]$ \\
\hline 4 Self-efficacy & 3 & 108 & Std. Mean Difference (IV, Fixed, 95\% CI) & $1.07[0.64,1.49]$ \\
\hline
\end{tabular}

Comparison 2. Structured goal setting (with or without strategies to enhance goal pursuit) versus no structured goal setting

\begin{tabular}{|c|c|c|c|c|}
\hline Outcome or subgroup title & $\begin{array}{l}\text { No. of } \\
\text { studies }\end{array}$ & $\begin{array}{c}\text { No. of } \\
\text { participants }\end{array}$ & Statistical method & Effect size \\
\hline $\begin{array}{l}1 \text { Health related quality of life or } \\
\text { self-reported emotional status }\end{array}$ & 5 & 441 & Std. Mean Difference (IV, Random, 95\% CI) & $0.18[-0.19,0.56]$ \\
\hline 2 Activity - ability & 4 & 277 & Std. Mean Difference (IV, Random, 95\% CI) & $0.17[-0.15,0.49]$ \\
\hline 3 Self-efficacy & 2 & 134 & Std. Mean Difference (IV, Fixed, 95\% CI) & $0.37[0.02,0.71]$ \\
\hline 4 Satisfaction with service delivery & 5 & 309 & Std. Mean Difference (IV, Random, 95\% CI) & $0.33[0.10,0.56]$ \\
\hline $\begin{array}{l}5 \text { Adverse events (withdrawal due } \\
\text { to death, re-hospitalisation or } \\
\text { worsening symptoms) }\end{array}$ & 3 & 406 & Peto Odds Ratio (Peto, Fixed, 95\% CI) & $0.64[0.27,1.47]$ \\
\hline
\end{tabular}


Analysis I.I. Comparison I Goal setting (with or without strategies to enhance goal pursuit) versus no goal setting, Outcome I Health related quality of life or self-reported emotional status.

Review: Goal setting and strategies to enhance goal pursuit for adults with acquired disability participating in rehabilitation

Comparison: I Goal setting (with or without strategies to enhance goal pursuit) versus no goal setting

Outcome: I Health related quality of life or self-reported emotional status

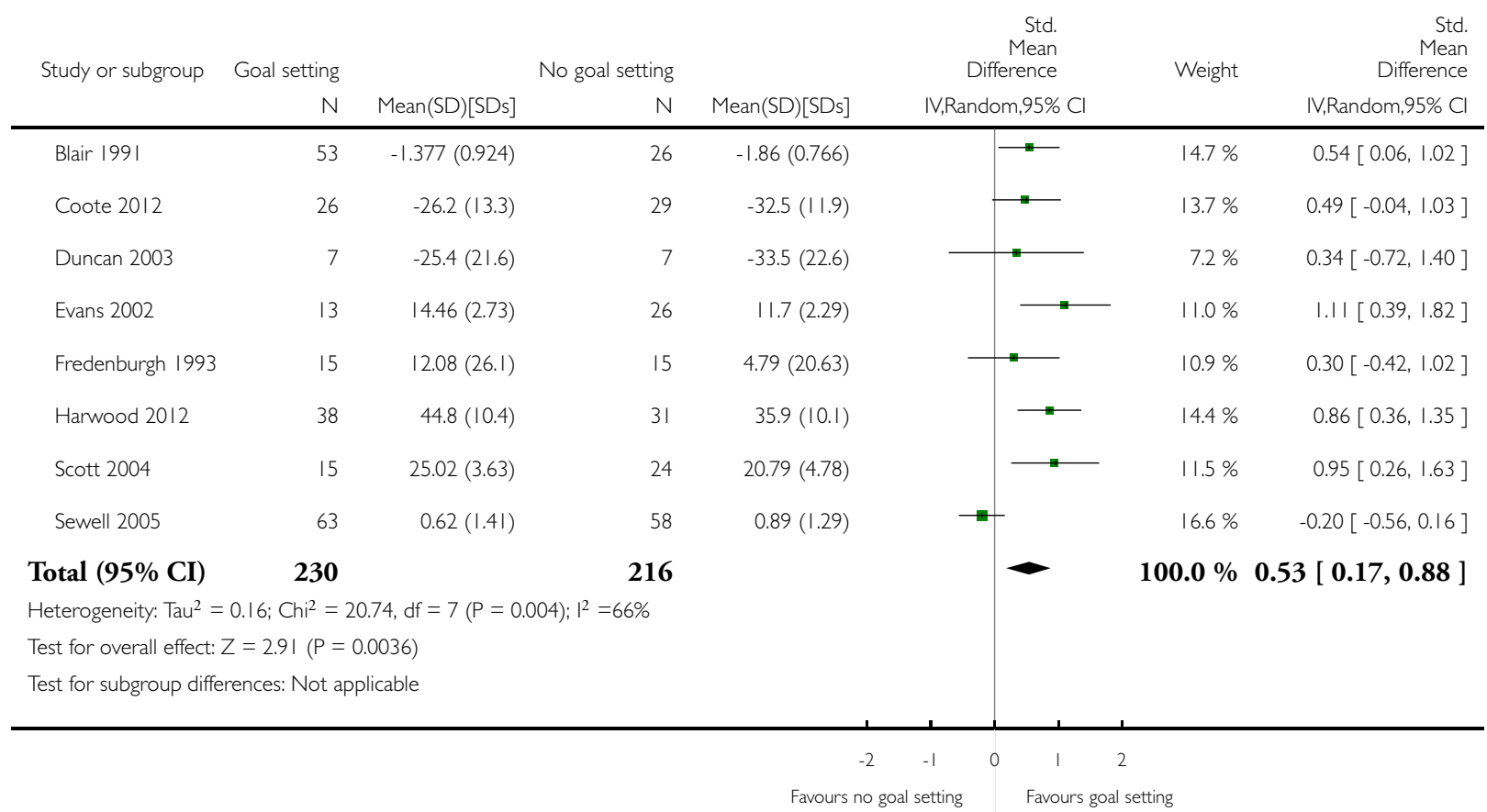


Analysis I.2. Comparison I Goal setting (with or without strategies to enhance goal pursuit) versus no goal setting, Outcome 2 Activity - ability.

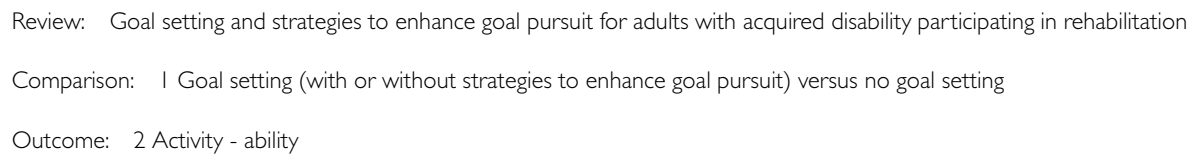

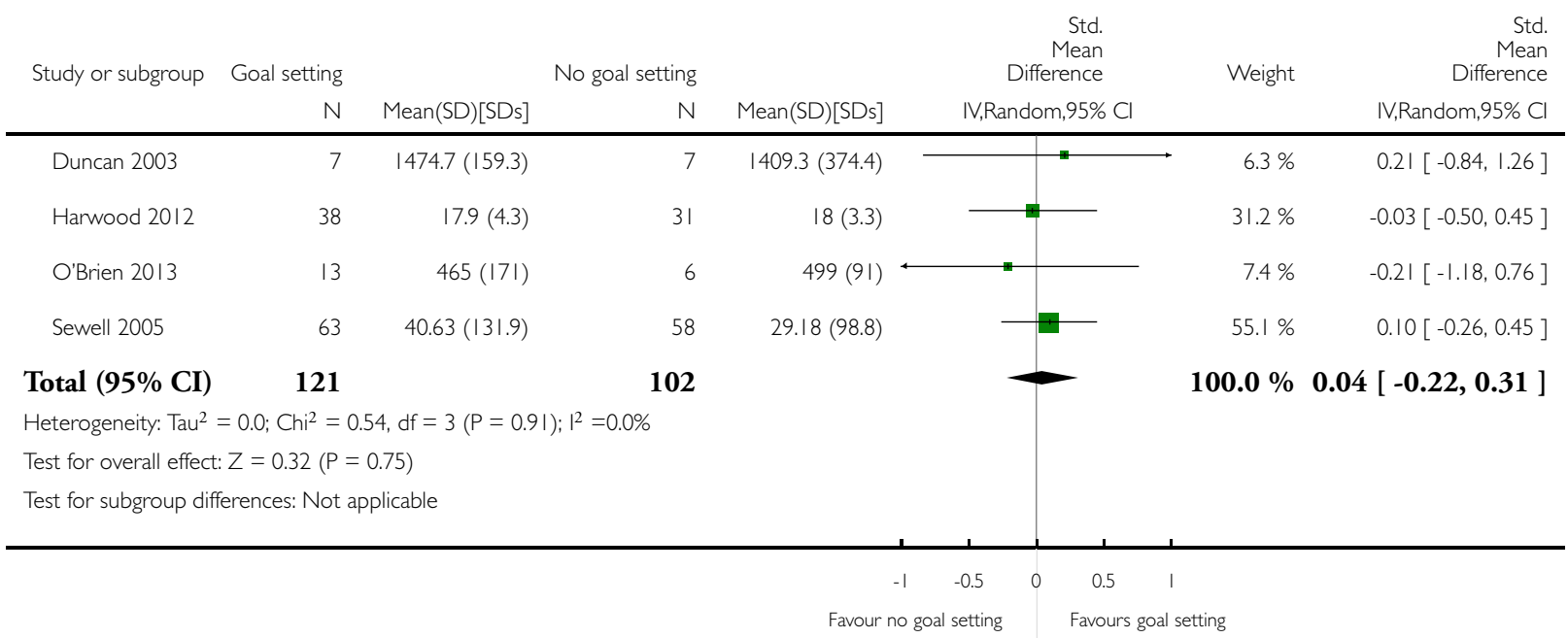


Analysis I.3. Comparison I Goal setting (with or without strategies to enhance goal pursuit) versus no goal setting, Outcome 3 Engagement in rehabilitation.

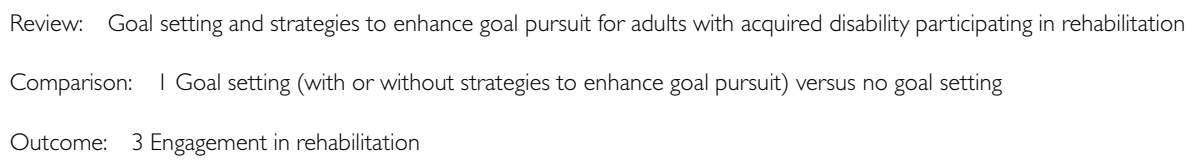

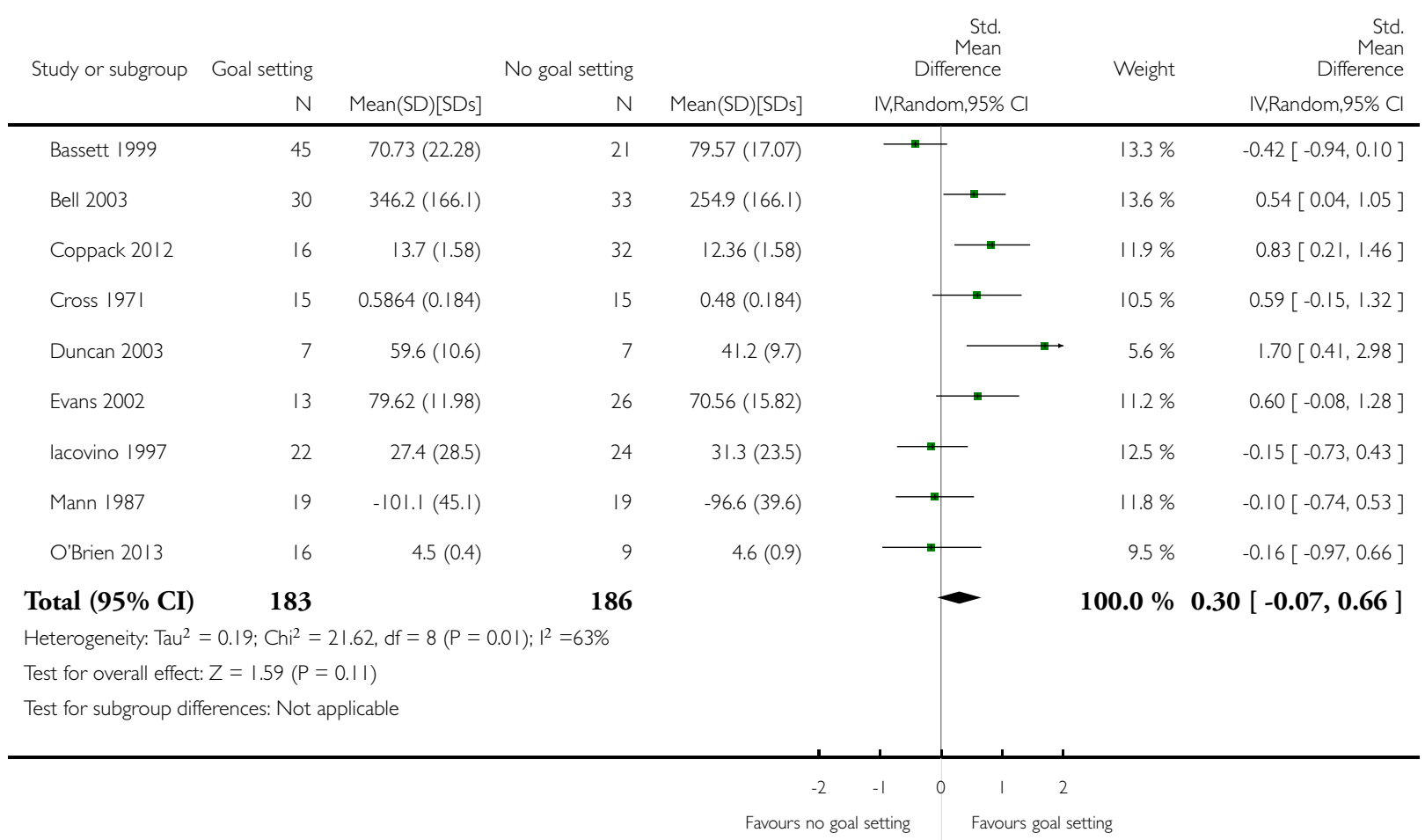


Analysis I.4. Comparison I Goal setting (with or without strategies to enhance goal pursuit) versus no goal setting, Outcome 4 Self-efficacy.

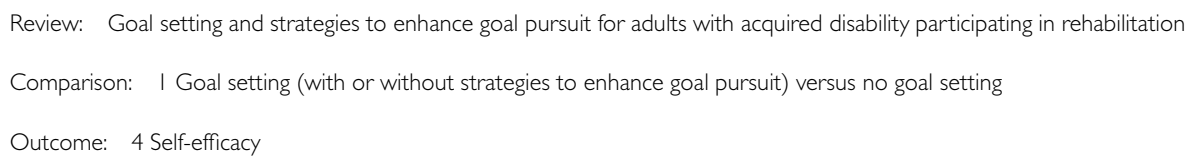

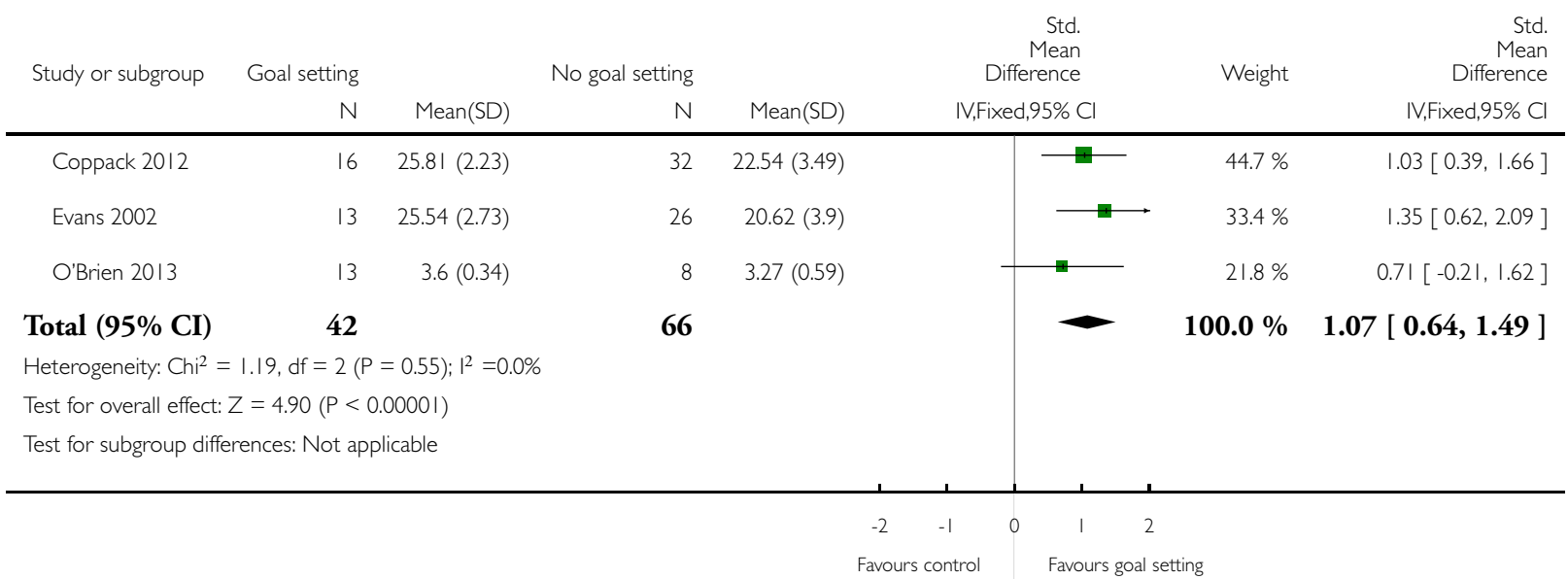


Analysis 2.1. Comparison 2 Structured goal setting (with or without strategies to enhance goal pursuit) versus no structured goal setting, Outcome I Health related quality of life or self-reported emotional status.

Review: Goal setting and strategies to enhance goal pursuit for adults with acquired disability participating in rehabilitation

Comparison: 2 Structured goal setting (with or without strategies to enhance goal pursuit) versus no structured goal setting

Outcome: I Health related quality of life or self-reported emotional status

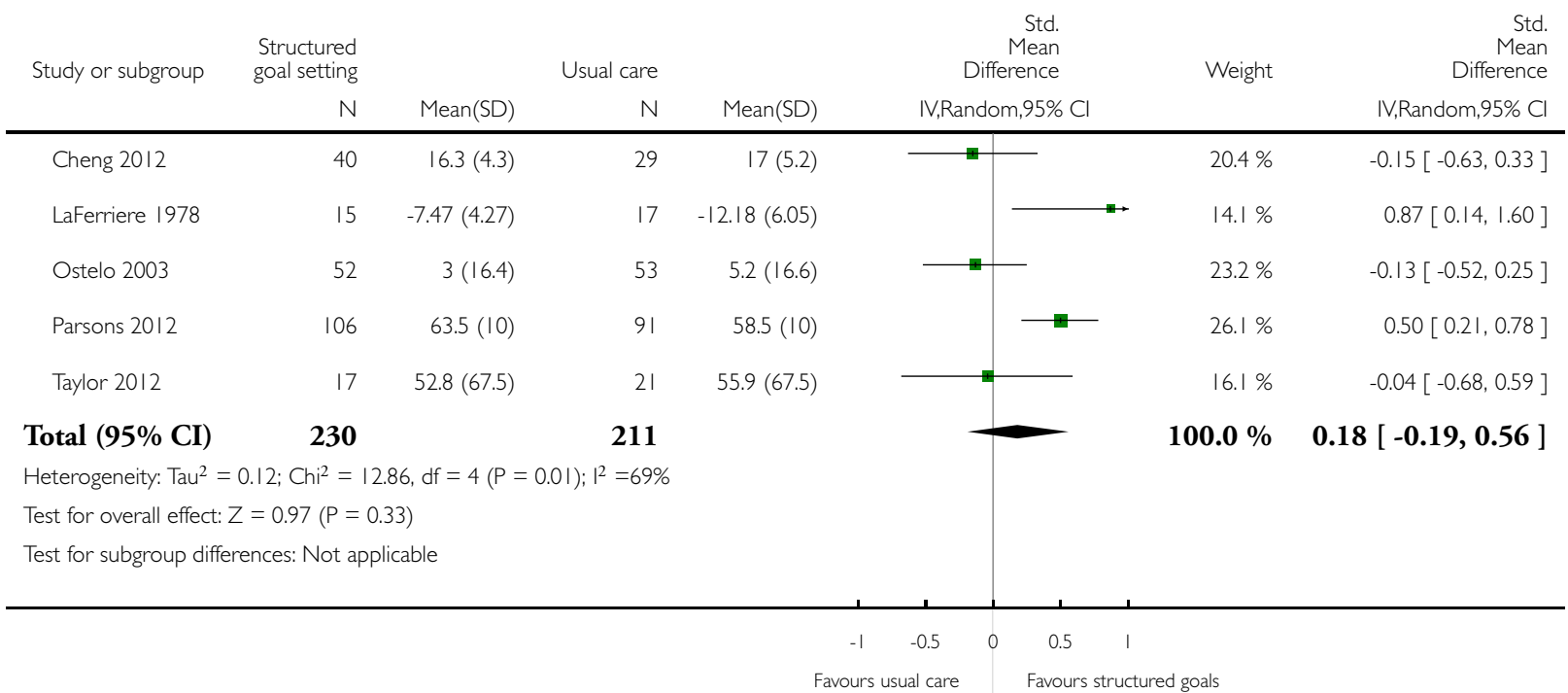


Analysis 2.2. Comparison 2 Structured goal setting (with or without strategies to enhance goal pursuit) versus no structured goal setting, Outcome 2 Activity - ability.

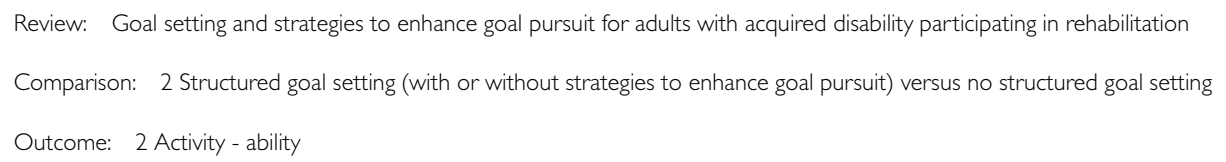

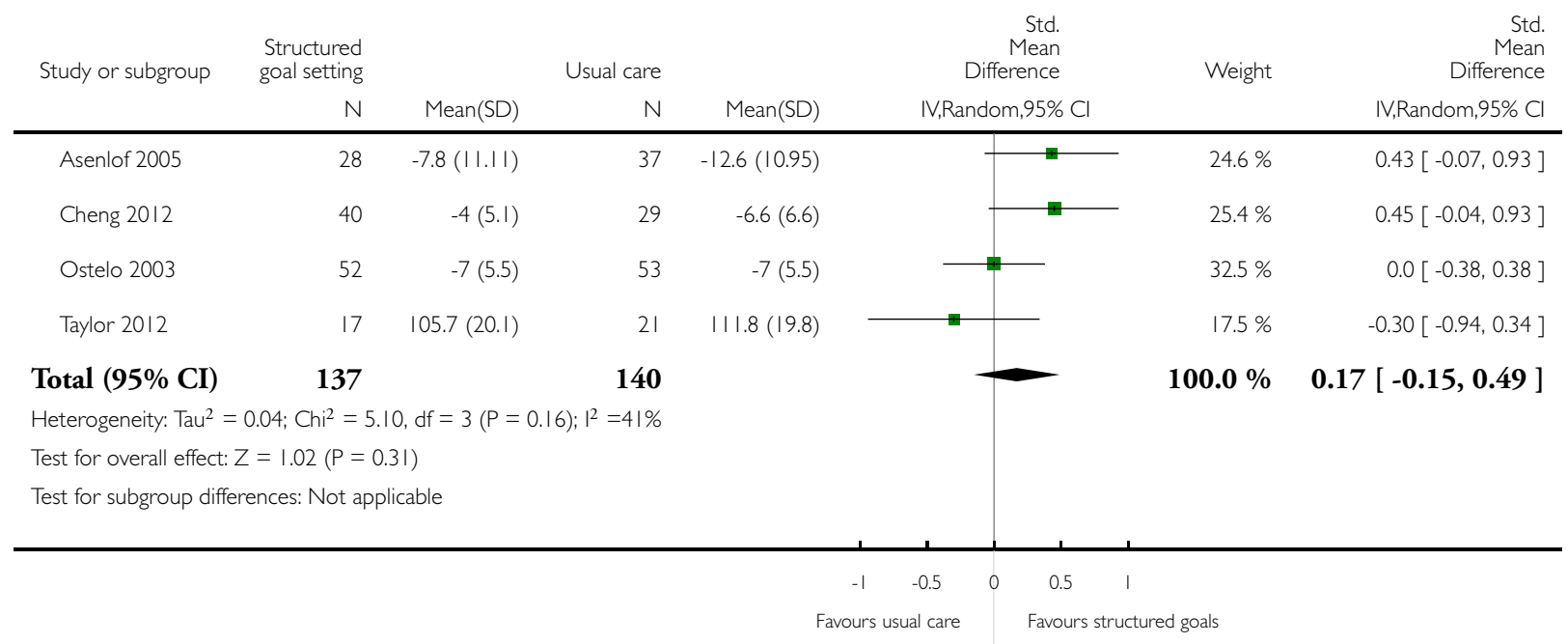


Analysis 2.3. Comparison 2 Structured goal setting (with or without strategies to enhance goal pursuit) versus no structured goal setting, Outcome 3 Self-efficacy.

Review: Goal setting and strategies to enhance goal pursuit for adults with acquired disability participating in rehabilitation

Comparison: 2 Structured goal setting (with or without strategies to enhance goal pursuit) versus no structured goal setting

Outcome: 3 Self-efficacy

\begin{tabular}{|c|c|c|c|c|c|c|c|}
\hline \multirow[t]{2}{*}{ Study or subgroup } & \multirow{2}{*}{$\begin{array}{r}\text { Structured } \\
\text { goal setting } \\
N\end{array}$} & \multicolumn{3}{|c|}{ Usual care } & \multirow{2}{*}{$\begin{array}{r}\text { Std. } \\
\text { Mean } \\
\text { Difference } \\
\text { IV,Fixed,95\% Cl }\end{array}$} & \multirow[t]{2}{*}{ Weight } & \multirow{2}{*}{$\begin{array}{r}\text { Std. } \\
\text { Mean } \\
\text { Difference } \\
\text { IV,Fixed,95\% Cl }\end{array}$} \\
\hline & & Mean(SD) & $N$ & Mean(SD) & & & \\
\hline Asenlof 2005 & 28 & I79.8 (29.63) & 37 & I $68.6(29.81)$ & 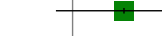 & $48.6 \%$ & $0.37[-0.12,0.87]$ \\
\hline Cheng 2012 & 40 & |5.| (1.8) & 29 & | $4.2(3.2)$ & 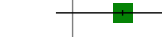 & $51.4 \%$ & $0.36[-0.12,0.84]$ \\
\hline
\end{tabular}

Total (95\% CI)

68

66

$100.0 \% \quad 0.37[0.02,0.71]$

Heterogeneity: $\mathrm{Chi}^{2}=0.00, \mathrm{df}=\mathrm{I}(\mathrm{P}=0.97) ; \mathrm{I}^{2}=0.0 \%$

Test for overall effect: $Z=2.07(P=0.038)$

Test for subgroup differences: Not applicable

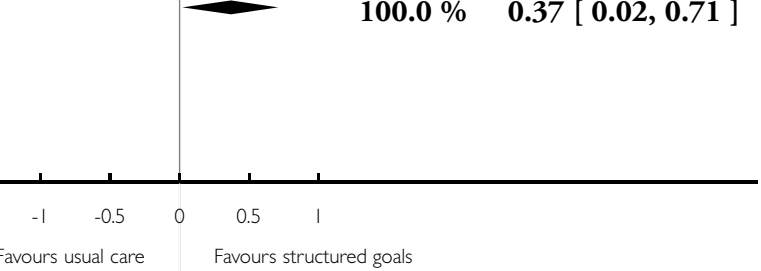


Analysis 2.4. Comparison 2 Structured goal setting (with or without strategies to enhance goal pursuit) versus no structured goal setting, Outcome 4 Satisfaction with service delivery.

Review: Goal setting and strategies to enhance goal pursuit for adults with acquired disability participating in rehabilitation

Comparison: 2 Structured goal setting (with or without strategies to enhance goal pursuit) versus no structured goal setting

Outcome: 4 Satisfaction with service delivery

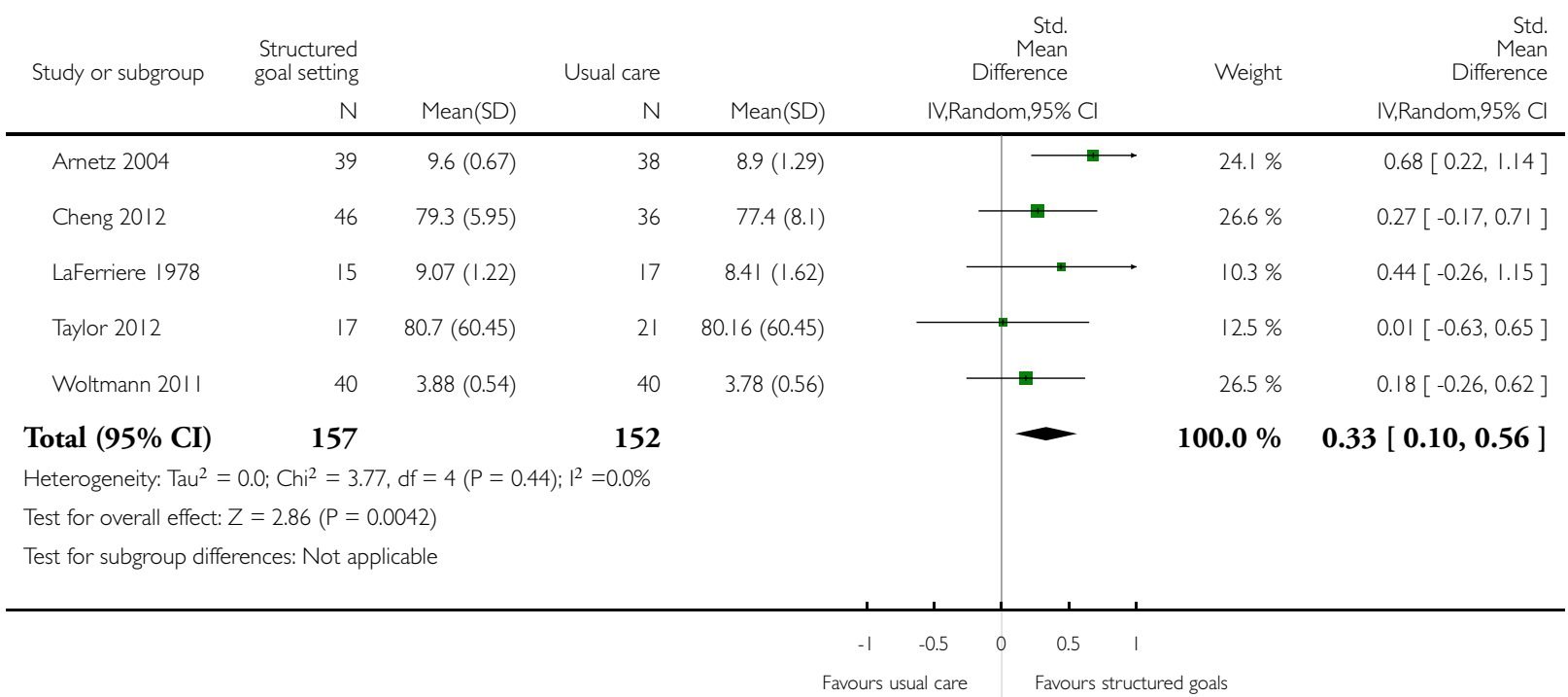


Analysis 2.5. Comparison 2 Structured goal setting (with or without strategies to enhance goal pursuit) versus no structured goal setting, Outcome 5 Adverse events (withdrawal due to death, re-hospitalisation or worsening symptoms).

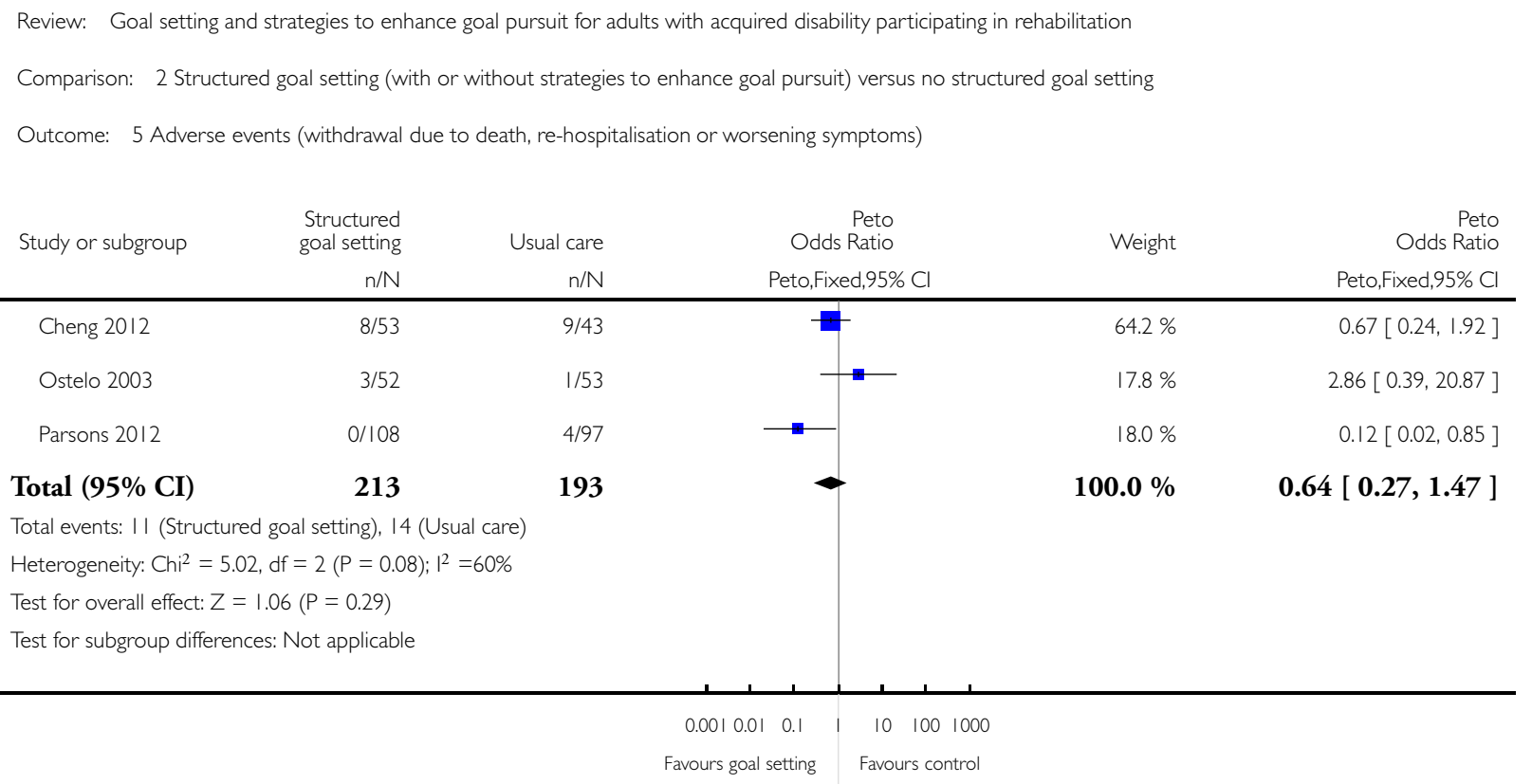

\section{ADDITIONAL TABLES}

Table 1. Included studies reported in multiple publications

\begin{tabular}{ll}
\hline Study & Other papers reporting study \\
\hline Asenlof 2005 & Asenlof 2006; Asenlof 2009 \\
\hline Blair 1991 & Blair 1995 \\
\hline Duncan 2003 & Duncan 2002 \\
\hline Jonsdottir 2012 & Jonsdottir 2012b \\
\hline Ostelo 2003 & Ostelo 2000; Ostelo 2004 \\
\hline Scott 2004 & Ranta 2000; Setter-Kline 2007; Watson 2001 \\
\hline Sewell 2005 & Sewell 2001 \\
\hline
\end{tabular}


Table 2. Management of outcome data for meta-analyses

Comparison 1: Structured goal setting with or without strategies to enhance goal pursuit versus no goal setting

Health-related quality of life and self-reported emotional status

\begin{tabular}{|c|c|c|c|c|c|}
\hline Study & Outcome measure & $\begin{array}{l}\text { Pooled in meta- } \\
\text { analysis? }\end{array}$ & $\begin{array}{l}\text { Se- } \\
\text { lection from multi- } \\
\text { ple measures and/ } \\
\text { or time points }\end{array}$ & $\begin{array}{l}\text { Management of } \\
\text { clustering }\end{array}$ & $\begin{array}{l}\text { Management of } \\
\text { multiple groups }\end{array}$ \\
\hline Bell 2003 & $\begin{array}{l}\text { Intrapsychic foun- } \\
\text { dation and Interper- } \\
\text { sonal function sub- } \\
\text { scales of the Quality } \\
\text { of Life Scale }\end{array}$ & $\begin{array}{l}\text { No, means and SDs } \\
\text { not reported }\end{array}$ & $\mathrm{n} / \mathrm{a}$ & $\mathrm{n} / \mathrm{a}$ & $\mathrm{n} / \mathrm{a}$ \\
\hline Blair 1991 & $\begin{array}{l}\text { Rosenberg Self-Es- } \\
\text { teem Scale }\end{array}$ & Yes & $\begin{array}{l}\mathrm{n} / \mathrm{a} \text { - only reported } \\
\text { on one measure at } \\
\text { one time point }\end{array}$ & $\begin{array}{l}\text { Unable to adjust for } \\
\text { effects of clustering; } \\
\text { influence of study } \\
\text { on findings tested in } \\
\text { sensitivity analysis }\end{array}$ & $\begin{array}{l}\text { Mutual goal setting } \\
\text { groups with } \\
\text { and without oper- } \\
\text { ant behaviour man- } \\
\text { agement were com- } \\
\text { bined into a single } \\
\text { experimental (goal } \\
\text { setting) group }\end{array}$ \\
\hline Coote 2012 & $\begin{array}{l}\text { Centre for Epidemi- } \\
\text { ological Studies-De- } \\
\text { pression Scale }\end{array}$ & Yes & $\begin{array}{l}\text { Lowest of the two } \\
\text { middle ranked ef- } \\
\text { fect estimates for } \\
\text { four possible mea- } \\
\text { sures (other possi- } \\
\text { ble measures: Sat- } \\
\text { isfaction with Life } \\
\text { Scale, Positive Af- } \\
\text { fect Scale, and Neg- } \\
\text { ative Affect Scale) }\end{array}$ & $\begin{array}{l}\text { n/a - not a cluster- } \\
\text { RCT }\end{array}$ & $\begin{array}{l}\text { n/a - only two study } \\
\text { groups }\end{array}$ \\
\hline Duncan 2003 & $\begin{array}{l}\text { Minnesota Living } \\
\text { with Heart Failure }\end{array}$ & Yes & $\begin{array}{l}\text { Only one measure } \\
\text { used but outcomes } \\
\text { from more than one } \\
\text { time point were re- } \\
\text { ported. Data from } \\
\text { the longest period } \\
\text { of time following re- } \\
\text { cruitment into the } \\
\text { study was included } \\
\text { in the meta-analysis }\end{array}$ & $\begin{array}{l}\text { n/a - not a cluster- } \\
\text { RCT }\end{array}$ & $\begin{array}{l}\mathrm{n} / \mathrm{a} \text { - only two study } \\
\text { groups }\end{array}$ \\
\hline Evans 2002 & $\begin{array}{l}\text { 'Reor- } \\
\text { ganisation' subscale } \\
\text { of the Psychological }\end{array}$ & Yes & $\begin{array}{l}\text { Only one measure } \\
\text { used but outcomes } \\
\text { from more than one }\end{array}$ & $\begin{array}{l}\text { n/a - not a cluster- } \\
\text { RCT }\end{array}$ & $\begin{array}{l}\text { The 'attention' con- } \\
\text { trol group (receiv- } \\
\text { ing social support }\end{array}$ \\
\hline
\end{tabular}


Table 2. Management of outcome data for meta-analyses (Continued)

\begin{tabular}{|c|c|c|c|c|c|}
\hline & Responses to Injury & & $\begin{array}{l}\text { time point were re- } \\
\text { ported. Data from } \\
\text { the longest period } \\
\text { of time following re- } \\
\text { cruitment into the } \\
\text { study was included } \\
\text { in the meta-analysis }\end{array}$ & & $\begin{array}{l}\text { but not goal setting } \\
\text { from a sport psy- } \\
\text { chologist) and the } \\
\text { 'no additional in- } \\
\text { put' } \\
\text { control group were } \\
\text { combined into a sin- } \\
\text { gle control group }\end{array}$ \\
\hline Fredenburgh 1993 & $\begin{array}{l}\text { Derogatis Stress } \\
\text { Profile }\end{array}$ & Yes & $\begin{array}{l}\mathrm{n} / \mathrm{a} \text { - only reported } \\
\text { on one measure at } \\
\text { one time point }\end{array}$ & $\begin{array}{l}\text { n/a - not a cluster- } \\
\text { RCT }\end{array}$ & $\begin{array}{l}\text { n/a - only two study } \\
\text { groups }\end{array}$ \\
\hline Harwood 2012 & $\begin{array}{l}\text { Physical } \\
\text { Component Sum- } \\
\text { mary scores from } \\
\text { the 36-Item Short } \\
\text { Form Health Survey }\end{array}$ & Yes & $\begin{array}{l}\text { Selected as this was } \\
\text { the primary out- } \\
\text { come measure used } \\
\text { in the sample size } \\
\text { calculation }\end{array}$ & $\begin{array}{l}\text { n/a - not a cluster- } \\
\text { RCT }\end{array}$ & $\begin{array}{l}\text { Four study groups. } \\
\text { However, we judged } \\
\text { the DVD inspira- } \\
\text { tional video to be } \\
\text { a separate interven- } \\
\text { tion unrelated to } \\
\text { goal setting, so only } \\
\text { included data from } \\
\text { the 'Take Charge' } \\
\text { (goal setting) inter- } \\
\text { vention as experi- } \\
\text { mental group data } \\
\text { and data from the } \\
\text { 'usual care' group as } \\
\text { control group data }\end{array}$ \\
\hline Iacovino 1997 & $\begin{array}{l}\text { Satisfaction with } \\
\text { Life Scale }\end{array}$ & $\begin{array}{l}\text { No, means and SDs } \\
\text { not reported }\end{array}$ & $\mathrm{n} / \mathrm{a}$ & $\mathrm{n} / \mathrm{a}$ & $\mathrm{n} / \mathrm{a}$ \\
\hline Scott 2004 & $\begin{array}{l}\text { Cardiac version of } \\
\text { the Quality of Life } \\
\text { Index }\end{array}$ & Yes & $\begin{array}{l}\text { Lowest effect esti- } \\
\text { mate from two pos- } \\
\text { sible measures } \\
\text { (other possible mea- } \\
\text { sure: Mental Health } \\
\text { Inventory-5) }\end{array}$ & $\begin{array}{l}\text { n/a - not a cluster- } \\
\text { RCT }\end{array}$ & $\begin{array}{l}\text { Three study groups. } \\
\text { However, we judged } \\
\text { the self- } \\
\text { management educa- } \\
\text { tion to be a sepa- } \\
\text { rate intervention, so } \\
\text { excluded data from } \\
\text { this group from our } \\
\text { analysis }\end{array}$ \\
\hline Sewell 2005 & $\begin{array}{l}\text { Dys- } \\
\text { pnea component of } \\
\text { the Chronic Res- } \\
\text { piratory Question- } \\
\text { naire }\end{array}$ & Yes & $\begin{array}{l}\text { Lowest of the two } \\
\text { middle ranked ef- } \\
\text { fect estimates for } \\
\text { four possible mea- } \\
\text { sures (other possible } \\
\text { mea- } \\
\text { sures: the Fatigue, }\end{array}$ & $\begin{array}{l}\text { n/a - not a cluster- } \\
\text { RCT }\end{array}$ & $\begin{array}{l}\mathrm{n} / \mathrm{a} \text { - only two study } \\
\text { groups }\end{array}$ \\
\hline
\end{tabular}

Goal setting and strategies to enhance goal pursuit for adults with acquired disability participating in rehabilitation (Review) 
Table 2. Management of outcome data for meta-analyses (Continued)

Emotion, and Mastery components of the Chronic Respiratory Questionnaire)

Activity - ability

\begin{tabular}{|c|c|c|c|c|c|}
\hline Study & Outcome measure & $\begin{array}{l}\text { Pooled in meta- } \\
\text { analysis? }\end{array}$ & $\begin{array}{l}\text { Se- } \\
\text { lection from multi- } \\
\text { ple measures and/ } \\
\text { or time points }\end{array}$ & $\begin{array}{l}\text { Management of } \\
\text { clustering }\end{array}$ & $\begin{array}{l}\text { Management of } \\
\text { multiple groups }\end{array}$ \\
\hline Duncan 2003 & $\begin{array}{l}\text { Six Minute Walk } \\
\text { Test }\end{array}$ & Yes & $\begin{array}{l}\text { Only one measure } \\
\text { used but outcomes } \\
\text { from more than one } \\
\text { time point were re- } \\
\text { ported. Data from } \\
\text { the longest period } \\
\text { of time following re- } \\
\text { cruitment into the } \\
\text { study was included } \\
\text { in the meta-analysis }\end{array}$ & $\begin{array}{l}\text { n/a - not a cluster- } \\
\text { RCT }\end{array}$ & $\begin{array}{l}\mathrm{n} / \mathrm{a} \text { - only two study } \\
\text { groups }\end{array}$ \\
\hline Harwood 2012 & Barthel Index & Yes & $\begin{array}{l}\text { Lowest effect esti- } \\
\text { mate from two pos- } \\
\text { sible measures } \\
\text { (other possible mea- } \\
\text { sure: Frenchay Ac- } \\
\text { tivities Index) }\end{array}$ & $\begin{array}{l}\mathrm{n} / \mathrm{a}-\text { not a cluster- } \\
\text { RCT }\end{array}$ & $\begin{array}{l}\text { Four study groups. } \\
\text { However, we judged } \\
\text { the DVD inspira- } \\
\text { tional video to be } \\
\text { a separate interven- } \\
\text { tion unrelated to } \\
\text { goal setting, so only } \\
\text { included data from } \\
\text { the 'Take Charge' } \\
\text { (goal setting) inter- } \\
\text { vention as experi- } \\
\text { mental group data } \\
\text { and data from the } \\
\text { 'usual care' group as } \\
\text { control group data }\end{array}$ \\
\hline O'Brien 2013 & $\begin{array}{l}\text { Six Minute Walk } \\
\text { Test }\end{array}$ & Yes & $\begin{array}{l}\text { Median effect esti- } \\
\text { mate out } \\
\text { of five possible mea- } \\
\text { sures (other possi- } \\
\text { ble measures: Timed } \\
\text { Up and Go; Activity } \\
\text { of Daily Living sub- } \\
\text { scale of the Lower } \\
\text { Limb Task Ques- }\end{array}$ & $\begin{array}{l}\text { n/a - not a cluster- } \\
\text { RCT }\end{array}$ & $\begin{array}{l}\mathrm{n} / \mathrm{a} \text { - only two study } \\
\text { groups }\end{array}$ \\
\hline
\end{tabular}


Table 2. Management of outcome data for meta-analyses (Continued)

tionnaire; Step Test;

Ten meter Walk Test

\begin{tabular}{l|l|l|l}
\hline Sewell 2005 & $\begin{array}{l}\text { Continuous ambu- } \\
\text { latory activity mon- } \\
\text { itor counts }\end{array}$ & $\begin{array}{l}\text { Selected as this was n/a - not a cluster- n/a - only two study } \\
\text { the primary out- RCT } \\
\text { come measure used } \\
\text { in the sample size } \\
\text { calculation }\end{array}$ \\
\hline
\end{tabular}

Engagement in rehabilitation

\begin{tabular}{|c|c|c|c|c|c|}
\hline Study & Outcome measure & $\begin{array}{l}\text { Pooled in meta- } \\
\text { analysis? }\end{array}$ & $\begin{array}{l}\text { Se- } \\
\text { lection from multi- } \\
\text { ple measures and/ } \\
\text { or time points }\end{array}$ & $\begin{array}{l}\text { Management of } \\
\text { clustering }\end{array}$ & $\begin{array}{l}\text { Management of } \\
\text { multiple groups }\end{array}$ \\
\hline Bassett 1999 & $\begin{array}{l}\text { Self-re- } \\
\text { ported percentage of } \\
\text { recommended exer- } \\
\text { cises session com- } \\
\text { pleted }\end{array}$ & Yes & $\begin{array}{l}\text { Lowest } \\
\text { effect estimate from } \\
\text { two possible mea- } \\
\text { sures (other possi- } \\
\text { ble measure: self-re- } \\
\text { ported percentage of } \\
\text { recommended repe- } \\
\text { titions of each exer- } \\
\text { cise completed) }\end{array}$ & $\begin{array}{l}\text { n/a - not a cluster- } \\
\text { RCT }\end{array}$ & $\begin{array}{l}\text { Three groups. We } \\
\text { combined } \\
\text { the two groups in- } \\
\text { volving participant- } \\
\text { physiotherapist col- } \\
\text { laborative goal set- } \\
\text { ting and physiother- } \\
\text { apist-mandated goal } \\
\text { setting into a single } \\
\text { experimental (goal } \\
\text { setting) group }\end{array}$ \\
\hline Bell 2003 & $\begin{array}{l}\text { Total num- } \\
\text { ber of hours worked } \\
\text { during a work trial }\end{array}$ & Yes & $\begin{array}{l}\text { Lowest effect esti- } \\
\text { mate from two pos- } \\
\text { sible measures } \\
\text { (other possible mea- } \\
\text { sure: Total num- } \\
\text { ber of weeks worked } \\
\text { during a work trial) }\end{array}$ & $\begin{array}{l}\text { n/a - not a cluster- } \\
\text { RCT }\end{array}$ & $\begin{array}{l}\mathrm{n} / \mathrm{a} \text { - only two study } \\
\text { groups }\end{array}$ \\
\hline Coppack 2012 & $\begin{array}{l}\text { Sports Injury Re- } \\
\text { habilitation Adher- } \\
\text { ence Scale }\end{array}$ & Yes & $\begin{array}{l}\mathrm{n} / \mathrm{a} \text { - only reported } \\
\text { on one measure at } \\
\text { one time point }\end{array}$ & $\begin{array}{l}\text { n/a - not a cluster- } \\
\text { RCT }\end{array}$ & $\begin{array}{l}\text { Three groups. We } \\
\text { com- } \\
\text { bined the groups } \\
\text { involving therapist- } \\
\text { directed exercise } \\
\text { without goal set- } \\
\text { ting and non-ther- } \\
\text { apist-directed exer- } \\
\text { cise without goal } \\
\text { setting into a single } \\
\text { control group }\end{array}$ \\
\hline
\end{tabular}

Goal setting and strategies to enhance goal pursuit for adults with acquired disability participating in rehabilitation (Review) 
Table 2. Management of outcome data for meta-analyses (Continued)

\begin{tabular}{|c|c|c|c|c|c|}
\hline Cross 1971 & $\begin{array}{l}\text { Percentage } \\
\text { adherence to recom- } \\
\text { mended food selec- } \\
\text { tion }\end{array}$ & Yes & $\begin{array}{l}\mathrm{n} / \mathrm{a} \text { - only reported } \\
\text { on one measure }\end{array}$ & $\begin{array}{l}\text { n/a - not a cluster- } \\
\text { RCT }\end{array}$ & $\begin{array}{l}\text { Three groups. How- } \\
\text { ever, we judged the } \\
\text { group receiving no } \\
\text { dietary education as } \\
\text { irrelevant to the re- } \\
\text { view question, so } \\
\text { only included } \\
\text { the groups receiv- } \\
\text { ing dietary educa- } \\
\text { tion with and with- } \\
\text { out goal setting }\end{array}$ \\
\hline Duncan 2003 & $\begin{array}{l}\text { Number of recom- } \\
\text { mended exercise ses- } \\
\text { sions completed }\end{array}$ & Yes & $\begin{array}{l}\text { Only one measure } \\
\text { used but outcomes } \\
\text { from more than one } \\
\text { time point were re- } \\
\text { ported. Data from } \\
\text { the longest period } \\
\text { of time following re- } \\
\text { cruitment into the } \\
\text { study was included } \\
\text { in the meta-analysis }\end{array}$ & $\begin{array}{l}\text { n/a - not a cluster- } \\
\text { RCT }\end{array}$ & $\begin{array}{l}\text { n/a - only two study } \\
\text { groups }\end{array}$ \\
\hline Evans 2002 & $\begin{array}{l}\text { Overall physiother- } \\
\text { apist estimate of ad- } \\
\text { herence }\end{array}$ & Yes & $\begin{array}{l}\text { Lowest } \\
\text { effect estimate from } \\
\text { two possible mea- } \\
\text { sures (other possi- } \\
\text { ble measure: self-re- } \\
\text { ported percentage of } \\
\text { recommended exer- } \\
\text { cises completed) }\end{array}$ & $\begin{array}{l}\text { n/a - not a cluster- } \\
\text { RCT }\end{array}$ & $\begin{array}{l}\text { Three groups. We } \\
\text { combined the 'at- } \\
\text { tention' control } \\
\text { group (receiving so- } \\
\text { cial support but not } \\
\text { goal setting from a } \\
\text { sport psychologist) } \\
\text { and the 'no addi- } \\
\text { tional input' control } \\
\text { group into a single } \\
\text { control group }\end{array}$ \\
\hline Howell 1986 & $\begin{array}{l}\text { 'Motiva- } \\
\text { tion' subscale of the } \\
\text { Griffiths Work Per- } \\
\text { formance Scale }\end{array}$ & $\begin{array}{l}\text { No, SDs not re- } \\
\text { ported }\end{array}$ & $\mathrm{n} / \mathrm{a}$ & $\mathrm{n} / \mathrm{a}$ & $\mathrm{n} / \mathrm{a}$ \\
\hline Iacovino 1997 & $\begin{array}{l}\text { Percentage of eligi- } \\
\text { ble weeks worked } \\
\text { during a work trial }\end{array}$ & Yes & $\begin{array}{l}\text { No data were pro- } \\
\text { vided on the other } \\
\text { measure referred to } \\
\text { in the study: the } \\
\text { Work Values Inven- } \\
\text { tory }\end{array}$ & $\begin{array}{l}\text { n/a - not a cluster- } \\
\text { RCT }\end{array}$ & $\begin{array}{l}\mathrm{n} / \mathrm{a} \text { - only two study } \\
\text { groups }\end{array}$ \\
\hline
\end{tabular}


Table 2. Management of outcome data for meta-analyses (Continued)

\begin{tabular}{|c|c|c|c|c|c|}
\hline Mann 1987 & $\begin{array}{l}24 \text {-hour self- } \\
\text { reported sodium in- } \\
\text { take }\end{array}$ & Yes & $\begin{array}{l}\text { Only one measure } \\
\text { used but outcomes } \\
\text { from more than one } \\
\text { time point were re- } \\
\text { ported. Data from } \\
\text { the longest period } \\
\text { of time following re- } \\
\text { cruitment into the } \\
\text { study was included } \\
\text { in the meta-analysis }\end{array}$ & $\begin{array}{l}\text { n/a - not a cluster- } \\
\text { RCT }\end{array}$ & $\begin{array}{l}\text { Three groups. How- } \\
\text { ever, we judged the } \\
\text { group receiving no } \\
\text { dietary education as } \\
\text { irrelevant to the re- } \\
\text { view question, so } \\
\text { only included } \\
\text { the groups receiv- } \\
\text { ing dietary educa- } \\
\text { tion with and with- } \\
\text { out goal setting }\end{array}$ \\
\hline O’Brien 2013 & $\begin{array}{l}\text { Sports Injury Re- } \\
\text { habilitation Adher- } \\
\text { ence Scale }\end{array}$ & Yes & $\begin{array}{l}\text { Lowest of the two } \\
\text { middle ranked ef- } \\
\text { fect estimates for } \\
\text { four possible mea- } \\
\text { sures (other possi- } \\
\text { ble measures: num- } \\
\text { ber of classes at- } \\
\text { tended; self-re- } \\
\text { ported adherence to } \\
\text { stretching exercises; } \\
\text { self-reported adher- } \\
\text { ence to walking ex- } \\
\text { ercises) }\end{array}$ & $\begin{array}{l}\text { n/a - not a cluster- } \\
\text { RCT }\end{array}$ & $\begin{array}{l}\text { n/a - only two study } \\
\text { groups }\end{array}$ \\
\hline \multicolumn{6}{|l|}{ Self-efficacy } \\
\hline Study & Outcome measure & $\begin{array}{l}\text { Pooled in meta- } \\
\text { analysis? }\end{array}$ & $\begin{array}{l}\text { Se- } \\
\text { lection from multi- } \\
\text { ple measures and/ } \\
\text { or time points }\end{array}$ & $\begin{array}{l}\text { Management of } \\
\text { clustering }\end{array}$ & $\begin{array}{l}\text { Management of } \\
\text { multiple groups }\end{array}$ \\
\hline Coppack 2012 & $\begin{array}{l}\text { Sports Injury Re- } \\
\text { habilitation Beliefs } \\
\text { Survey }\end{array}$ & Yes & $\begin{array}{l}\mathrm{n} / \mathrm{a} \text { - only reported } \\
\text { on one measure at } \\
\text { one time point }\end{array}$ & $\begin{array}{l}\text { n/a - not a cluster- } \\
\text { RCT }\end{array}$ & $\begin{array}{l}\text { Three groups. We } \\
\text { com- } \\
\text { bined the groups } \\
\text { involving therapist- } \\
\text { directed exercise } \\
\text { without goal set- } \\
\text { ting and non-ther- } \\
\text { apist-directed exer- } \\
\text { cise without goal } \\
\text { setting into a single } \\
\text { control group }\end{array}$ \\
\hline Evans 2002 & $\begin{array}{l}\text { Sports Injury Re- } \\
\text { habilitation Beliefs } \\
\text { Survey }\end{array}$ & Yes & $\begin{array}{l}\text { Only one measure } \\
\text { used but outcomes } \\
\text { from more than one }\end{array}$ & $\begin{array}{l}\mathrm{n} / \mathrm{a} \text { - not a cluster- } \\
\mathrm{RCT}\end{array}$ & $\begin{array}{l}\text { Three groups. We } \\
\text { combined the 'at- } \\
\text { tention' control }\end{array}$ \\
\hline
\end{tabular}

Goal setting and strategies to enhance goal pursuit for adults with acquired disability participating in rehabilitation (Review) 
Table 2. Management of outcome data for meta-analyses (Continued)

\begin{tabular}{|c|c|c|c|c|c|}
\hline & & & $\begin{array}{l}\text { time point were re- } \\
\text { ported. Data from } \\
\text { the longest period } \\
\text { of time following re- } \\
\text { cruitment into the } \\
\text { study was included } \\
\text { in the meta-analysis }\end{array}$ & & $\begin{array}{l}\text { group (receiving so- } \\
\text { cial support but not } \\
\text { goal setting from a } \\
\text { sport psychologist) } \\
\text { and the 'no addi- } \\
\text { tional input' control } \\
\text { group into a single } \\
\text { control group }\end{array}$ \\
\hline O'Brien 2013 & Task self-efficacy & Yes & $\begin{array}{l}\text { Median of three ef- } \\
\text { fect estimates (other } \\
\text { possible measures: } \\
\text { maintenance self-ef- } \\
\text { ficacy, recovery self- } \\
\text { efficacy) }\end{array}$ & $\begin{array}{l}\mathrm{n} / \mathrm{a} \text { - not a cluster- } \\
\mathrm{RCT}\end{array}$ & $\begin{array}{l}\mathrm{n} / \mathrm{a} \text { - only two study } \\
\text { groups }\end{array}$ \\
\hline Scott 2004 & $\begin{array}{l}\text { Self-effi- } \\
\text { cacy to Manage Dis- } \\
\text { ease in General }\end{array}$ & $\begin{array}{l}\text { No, SDs not re- } \\
\text { ported }\end{array}$ & $\mathrm{n} / \mathrm{a}$ & $\mathrm{n} / \mathrm{a}$ & $\mathrm{n} / \mathrm{a}$ \\
\hline
\end{tabular}

Comparison 2: Structured approach to goal setting with or without strategiesto enhance goal pursuit versus 'usual care' that may have involved some goal setting but where no structured approach was followed

Health-related quality of life and self-reported emotional status

\begin{tabular}{|c|c|c|c|c|c|}
\hline Study & Outcome measure & $\begin{array}{l}\text { Pooled in meta- } \\
\text { analysis? }\end{array}$ & $\begin{array}{l}\text { Se- } \\
\text { lection from multi- } \\
\text { ple measures and/ } \\
\text { or time points }\end{array}$ & $\begin{array}{l}\text { Management of } \\
\text { clustering }\end{array}$ & $\begin{array}{l}\text { Management of } \\
\text { multiple groups }\end{array}$ \\
\hline Cheng 2012 & $\begin{array}{l}\text { Classification Com- } \\
\text { mittee of the World } \\
\text { Organization of Na- } \\
\text { tional Colleges, } \\
\text { Academies and Aca- } \\
\text { demic Associations } \\
\text { of General Practi- } \\
\text { tioners/Family } \\
\text { Physicians' chart of } \\
\text { perceived health sta- } \\
\text { tus }\end{array}$ & Yes & $\begin{array}{l}\text { Only one measure } \\
\text { used but outcomes } \\
\text { from more than one } \\
\text { time point were re- } \\
\text { ported. Data from } \\
\text { the longest period } \\
\text { of time following re- } \\
\text { cruitment into the } \\
\text { study were included } \\
\text { in the meta-analysis }\end{array}$ & $\begin{array}{l}\text { Unable to adjust for } \\
\text { effects of clustering; } \\
\text { influence of study } \\
\text { on findings tested in } \\
\text { sensitivity analysis }\end{array}$ & $\begin{array}{l}\mathrm{n} / \mathrm{a} \text { - only two study } \\
\text { groups }\end{array}$ \\
\hline Holliday 2007 & $\begin{array}{l}\text { General Health } \\
\text { Questionnaire }\end{array}$ & $\begin{array}{l}\text { No, means and SDs } \\
\text { not reported }\end{array}$ & $\mathrm{n} / \mathrm{a}$ & $\mathrm{n} / \mathrm{a}$ & $\mathrm{n} / \mathrm{a}$ \\
\hline LaFerriere 1978 & $\begin{array}{l}\text { Modified version of } \\
\text { the Welsh Anxiety } \\
\text { Scale }\end{array}$ & Yes & $\begin{array}{l}\text { Lowest of the two } \\
\text { middle ranked ef- } \\
\text { fect estimates for } \\
\text { four possible mea- }\end{array}$ & $\begin{array}{l}\text { n/a - not a cluster- } \\
\text { RCT }\end{array}$ & $\begin{array}{l}\mathrm{n} / \mathrm{a} \text { - only two study } \\
\text { groups }\end{array}$ \\
\hline
\end{tabular}

Goal setting and strategies to enhance goal pursuit for adults with acquired disability participating in rehabilitation (Review) 


\begin{tabular}{|c|c|c|c|c|c|}
\hline & & & $\begin{array}{l}\text { sures (other possible } \\
\text { mea- } \\
\text { sures: Anxiety Scale } \\
\text { of the Today form of } \\
\text { the Multiple Affect } \\
\text { Adjective Checklist; } \\
\text { Depression Scale of } \\
\text { the Today form of } \\
\text { the Multiple Affect } \\
\text { Adjective Checklist; } \\
\text { Rosenburg Self-es- } \\
\text { teem Scale) }\end{array}$ & & \\
\hline Ostelo 2003 & $\begin{array}{l}\text { General Health sub- } \\
\text { scale of the 36-Item } \\
\text { Short Form Health } \\
\text { Survey }\end{array}$ & Yes & $\begin{array}{l}\text { Lowest effect esti- } \\
\text { mate from two pos- } \\
\text { sible measures } \\
\text { (other possible mea- } \\
\text { sure: Social Func- } \\
\text { tioning subscale of } \\
\text { the } 36 \text {-Item Short } \\
\text { Form Health Sur- } \\
\text { vey) } \\
\text { Data were collected } \\
\text { from more than one } \\
\text { time point. Data } \\
\text { from the longest pe- } \\
\text { riod of time follow- } \\
\text { ing recruitment into } \\
\text { the study were in- } \\
\text { cluded in the meta- } \\
\text { analysis }\end{array}$ & $\begin{array}{l}\text { n/a - not a cluster- } \\
\text { RCT }\end{array}$ & $\begin{array}{l}\mathrm{n} / \mathrm{a} \text { - only two study } \\
\text { groups }\end{array}$ \\
\hline Parsons 2012 & $\begin{array}{l}\text { Mental Component } \\
\text { Summary score of } \\
\text { 36-Item Short Form } \\
\text { Health Survey }\end{array}$ & Yes & $\begin{array}{l}\text { Lowest effect esti- } \\
\text { mate from two pos- } \\
\text { sible measures } \\
\text { (other possible mea- } \\
\text { sure: Physical Com- } \\
\text { ponent Summary } \\
\text { score of the 36-Item } \\
\text { Short Form Health } \\
\text { Survey) }\end{array}$ & $\begin{array}{l}\text { Using the reported } \\
\text { data on mean differ- } \\
\text { ences between the } \\
\text { intervention } \\
\text { and control groups } \\
\text { with } 95 \% \text { CIs, we } \\
\text { calculated adjusted } \\
\text { means and SD that } \\
\text { reproduced the } 95 \% \\
\text { CI and P values in } \\
\text { the paper to account } \\
\text { for clustering }\end{array}$ & $\begin{array}{l}\text { n/a - only two study } \\
\text { groups }\end{array}$ \\
\hline Taylor 2012 & $\begin{array}{l}\text { Schedule for Evalu- } \\
\text { ation of Individual } \\
\text { Quality of Life }\end{array}$ & Yes & $\begin{array}{l}\text { Selected as this was } \\
\text { named the primary } \\
\text { outcome measure } \\
\text { (other possible mea- }\end{array}$ & $\begin{array}{l}\text { Using the reported } \\
\text { data on mean differ- } \\
\text { ences between the } \\
\text { intervention }\end{array}$ & $\begin{array}{l}\text { n/a - only two study } \\
\text { groups }\end{array}$ \\
\hline
\end{tabular}


sure: 36-Item Short Form Health Survey)

Data were collected from more than one time point. Data from the longest period of time following recruitment into the study were included in the metaanalysis and control groups with $95 \% \mathrm{CI}$, and the reported separate effects of clustering on variance plus the inter-class correlations for clusters, we calculated adjusted means and SD that reproduced the $95 \% \mathrm{CI}$ and $\mathrm{P}$ values in the paper to account for clustering

\section{Activity - ability}

\begin{tabular}{|c|c|c|c|c|c|}
\hline Study & Outcome measure & $\begin{array}{l}\text { Pooled in meta- } \\
\text { analysis? }\end{array}$ & $\begin{array}{l}\text { Se- } \\
\text { lection from multi- } \\
\text { ple measures and/ } \\
\text { or time points }\end{array}$ & $\begin{array}{l}\text { Management of } \\
\text { clustering }\end{array}$ & $\begin{array}{l}\text { Management of } \\
\text { multiple groups }\end{array}$ \\
\hline Asenlof 2005 & $\begin{array}{l}\text { Pain Disability In- } \\
\text { dex }\end{array}$ & Yes & $\begin{array}{l}\text { Only one measure } \\
\text { used but outcomes } \\
\text { from more than one } \\
\text { time point were re- } \\
\text { ported. Data from } \\
\text { the longest period } \\
\text { of time following re- } \\
\text { cruitment into the } \\
\text { study were included } \\
\text { in the meta-analysis }\end{array}$ & $\begin{array}{l}\text { n/a - not a cluster- } \\
\text { RCT }\end{array}$ & $\begin{array}{l}\mathrm{n} / \mathrm{a} \text { - only two study } \\
\text { groups }\end{array}$ \\
\hline Cheng 2012 & $\begin{array}{l}\text { Disability Index of } \\
\text { the Health Assess- } \\
\text { ment Questionnaire }\end{array}$ & Yes & $\begin{array}{l}\text { Only one measure } \\
\text { used but outcomes } \\
\text { from more than one } \\
\text { time point were re- } \\
\text { ported. Data from } \\
\text { the longest period } \\
\text { of time following re- } \\
\text { cruitment into the } \\
\text { study were included } \\
\text { in the meta-analysis }\end{array}$ & $\begin{array}{l}\text { Unable to adjust for } \\
\text { effects of clustering; } \\
\text { influence of study } \\
\text { on findings tested in } \\
\text { sensitivity analysis }\end{array}$ & $\begin{array}{l}\text { n/a - only two study } \\
\text { groups }\end{array}$ \\
\hline Holliday 2007 & $\begin{array}{l}\text { Functional Inde- } \\
\text { pendence Measure }\end{array}$ & $\begin{array}{l}\text { No, means and SDs } \\
\text { not reported }\end{array}$ & $\mathrm{n} / \mathrm{a}$ & $\mathrm{n} / \mathrm{a}$ & $\mathrm{n} / \mathrm{a}$ \\
\hline
\end{tabular}


Table 2. Management of outcome data for meta-analyses (Continued)

\begin{tabular}{|c|c|c|c|c|c|}
\hline Ostelo 2003 & $\begin{array}{l}\text { Roland Morris Dis- } \\
\text { ability } \\
\text { Questionnaire }\end{array}$ & Yes & $\begin{array}{l}\text { Lowest } \\
\text { effect estimate from } \\
\text { two possible mea- } \\
\text { sures (other possi- } \\
\text { ble measure: self-re- } \\
\text { ported severity of } \\
\text { main activity limi- } \\
\text { tation). Data were } \\
\text { collected from more } \\
\text { than one time point. } \\
\text { Data } \\
\text { from the longest pe- } \\
\text { riod of time follow- } \\
\text { ing recruitment into } \\
\text { the study were in- } \\
\text { cluded in the meta- } \\
\text { analysis }\end{array}$ & $\begin{array}{l}\text { n/a - not a cluster- } \\
\text { RCT }\end{array}$ & $\begin{array}{l}\mathrm{n} / \mathrm{a} \text { - only two study } \\
\text { groups }\end{array}$ \\
\hline Taylor 2012 & $\begin{array}{l}\text { Functional Inde- } \\
\text { pendence Measure }\end{array}$ & Yes & $\begin{array}{l}\text { Only one measure } \\
\text { used but outcomes } \\
\text { from more than one } \\
\text { time point were re- } \\
\text { ported. Data from } \\
\text { the longest period } \\
\text { of time following re- } \\
\text { cruitment into the } \\
\text { study were included } \\
\text { in the meta-analysis }\end{array}$ & $\begin{array}{l}\text { Using the reported } \\
\text { data on mean differ- } \\
\text { ences between the } \\
\text { intervention } \\
\text { and control groups } \\
\text { with } 95 \% \text { CIs, and } \\
\text { the reported sepa- } \\
\text { rate effects of clus- } \\
\text { tering on variance } \\
\text { plus the inter-class } \\
\text { correlations for clus- } \\
\text { ters, we calculated } \\
\text { adjusted means and } \\
\text { SD that reproduced } \\
\text { the } 95 \% \text { CI and P } \\
\text { values in the paper } \\
\text { to account for clus- } \\
\text { tering }\end{array}$ & $\begin{array}{l}\mathrm{n} / \mathrm{a} \text { - only two study } \\
\text { groups }\end{array}$ \\
\hline \multicolumn{6}{|l|}{ Self-efficacy } \\
\hline Study & Outcome measure & $\begin{array}{l}\text { Pooled in meta- } \\
\text { analysis? }\end{array}$ & $\begin{array}{l}\text { Se- } \\
\text { lection from multi- } \\
\text { ple measures and/ } \\
\text { or time points }\end{array}$ & $\begin{array}{l}\text { Management of } \\
\text { clustering }\end{array}$ & $\begin{array}{l}\text { Management of } \\
\text { multiple groups }\end{array}$ \\
\hline Asenlof 2005 & Self-efficacy Scale & Yes & $\begin{array}{l}\text { Only one measure } \\
\text { used but outcomes } \\
\text { from more than one } \\
\text { time point were re- }\end{array}$ & $\begin{array}{l}\text { n/a - not a cluster- } \\
\text { RCT }\end{array}$ & $\begin{array}{l}\mathrm{n} / \mathrm{a} \text { - only two study } \\
\text { groups }\end{array}$ \\
\hline
\end{tabular}

Goal setting and strategies to enhance goal pursuit for adults with acquired disability participating in rehabilitation (Review) 
Table 2. Management of outcome data for meta-analyses (Continued)

\begin{tabular}{|c|c|c|c|c|c|}
\hline & & & $\begin{array}{l}\text { ported. Data from } \\
\text { the longest period } \\
\text { of time following re- } \\
\text { cruitment into the } \\
\text { study were included } \\
\text { in the meta-analysis }\end{array}$ & & \\
\hline Cheng 2012 & $\begin{array}{l}\text { Chronic Disease } \\
\text { Self-efficacy Score }\end{array}$ & Yes & $\begin{array}{l}\text { Only one measure } \\
\text { used but outcomes } \\
\text { from more than one } \\
\text { time point were re- } \\
\text { ported. Data from } \\
\text { the longest period } \\
\text { of time following re- } \\
\text { cruitment into the } \\
\text { study were included } \\
\text { in the meta-analysis }\end{array}$ & $\begin{array}{l}\text { Unable to adjust for } \\
\text { effects of clustering }\end{array}$ & $\begin{array}{l}\mathrm{n} / \mathrm{a} \text { - only two study } \\
\text { groups }\end{array}$ \\
\hline
\end{tabular}

Satisfaction with service delivery

\begin{tabular}{|c|c|c|c|c|c|}
\hline Study & Outcome measure & $\begin{array}{l}\text { Pooled in meta- } \\
\text { analysis? }\end{array}$ & $\begin{array}{l}\text { Se- } \\
\text { lection from multi- } \\
\text { ple measures and/ } \\
\text { or time points }\end{array}$ & $\begin{array}{l}\text { Management of } \\
\text { clustering }\end{array}$ & $\begin{array}{l}\text { Management of } \\
\text { multiple groups }\end{array}$ \\
\hline Arnetz 2004 & $\begin{array}{l}\text { Overall quality of } \\
\text { physical therapy on } \\
\text { a } 0-10 \text { scale }\end{array}$ & Yes & $\begin{array}{l}\mathrm{n} / \mathrm{a} \text { - only reported } \\
\text { on one measure at } \\
\text { one time point }\end{array}$ & $\begin{array}{l}\text { n/a - not a cluster- } \\
\text { RCT }\end{array}$ & $\begin{array}{l}\mathrm{n} / \mathrm{a} \text { - only two study } \\
\text { groups }\end{array}$ \\
\hline Cheng 2012 & $\begin{array}{l}\text { Satisfac- } \\
\text { tion Scale for Com- } \\
\text { munity Nursing }\end{array}$ & Yes & $\begin{array}{l}\mathrm{n} / \mathrm{a} \text { - only reported } \\
\text { on one measure at } \\
\text { one time point }\end{array}$ & $\begin{array}{l}\text { Unable to adjust for } \\
\text { effects of clustering }\end{array}$ & $\begin{array}{l}\mathrm{n} / \mathrm{a} \text { - only two study } \\
\text { groups }\end{array}$ \\
\hline Holliday 2007 & $\begin{array}{l}\text { Overall satisfaction } \\
\text { on a } 10 \mathrm{~cm} \text { visual } \\
\text { analogue scale }\end{array}$ & $\begin{array}{l}\text { No, means and SDs } \\
\text { not reported }\end{array}$ & $\mathrm{n} / \mathrm{a}$ & $\mathrm{n} / \mathrm{a}$ & $\mathrm{n} / \mathrm{a}$ \\
\hline LaFerriere 1978 & $\begin{array}{l}\text { Satisfaction with } \\
\text { counselling }\end{array}$ & Yes & $\begin{array}{l}\mathrm{n} / \mathrm{a} \text { - only reported } \\
\text { on one measure at } \\
\text { one time point }\end{array}$ & $\begin{array}{l}\text { n/a - not a cluster- } \\
\text { RCT }\end{array}$ & $\begin{array}{l}\mathrm{n} / \mathrm{a} \text { - only two study } \\
\text { groups }\end{array}$ \\
\hline Taylor 2012 & $\begin{array}{l}\text { Patient Perception } \\
\text { of Rehabilitation }\end{array}$ & Yes & $\begin{array}{l}\text { Only one measure } \\
\text { used but outcomes } \\
\text { from more than one } \\
\text { time point were re- } \\
\text { ported. Data from } \\
\text { the longest period } \\
\text { of time following re- }\end{array}$ & $\begin{array}{l}\text { Using the reported } \\
\text { data on mean differ- } \\
\text { ences between } \\
\text { the intervention and } \\
\text { control groups with } \\
95 \% \text { CIs, and the } \\
\text { reported separate ef- }\end{array}$ & $\begin{array}{l}\mathrm{n} / \mathrm{a} \text { - only two study } \\
\text { groups }\end{array}$ \\
\hline
\end{tabular}

Goal setting and strategies to enhance goal pursuit for adults with acquired disability participating in rehabilitation (Review) 


\begin{tabular}{|c|c|c|c|c|c|}
\hline & & & $\begin{array}{l}\text { cruitment into the } \\
\text { study were included } \\
\text { in the meta-analysis }\end{array}$ & $\begin{array}{l}\text { fects of clustering on } \\
\text { variance plus the in- } \\
\text { ter-class correlations } \\
\text { for clusters (the lat- } \\
\text { ter accessed by } \\
\text { author communica- } \\
\text { tion), we calculated } \\
\text { adjusted means and } \\
\text { SD that reproduced } \\
\text { the } 95 \% \text { CI and P } \\
\text { values in the paper } \\
\text { to account for clus- } \\
\text { tering }\end{array}$ & \\
\hline Woltmann 2011 & $\begin{array}{l}\text { 5-point client sat- } \\
\text { isfaction question- } \\
\text { naire }\end{array}$ & Yes & $\begin{array}{l}\mathrm{n} / \mathrm{a} \text { - only reported } \\
\text { on one measure at } \\
\text { one time point }\end{array}$ & $\begin{array}{l}\mathrm{n} / \mathrm{a} \text { - not a cluster- } \\
\mathrm{RCT}\end{array}$ & $\begin{array}{l}\mathrm{n} / \mathrm{a} \text { - only two study } \\
\text { groups }\end{array}$ \\
\hline \multicolumn{6}{|c|}{ Adverse events (all-cause) } \\
\hline Study & Outcome measure & $\begin{array}{l}\text { Pooled in meta- } \\
\text { analysis? }\end{array}$ & $\begin{array}{l}\text { Se- } \\
\text { lection from multi- } \\
\text { ple measures and/ } \\
\text { or time points }\end{array}$ & $\begin{array}{l}\text { Management of } \\
\text { clustering }\end{array}$ & $\begin{array}{l}\text { Management of } \\
\text { multiple groups }\end{array}$ \\
\hline Cheng 2012 & $\begin{array}{l}\text { Number of par- } \\
\text { ticipants withdrawn } \\
\text { from the study due } \\
\text { to death or hospital- } \\
\text { isation }\end{array}$ & Yes & $\begin{array}{l}\text { With- } \\
\text { drawal due to death } \\
\text { or re-hospitalisation } \\
\text { combined. Num- } \\
\text { bers taken from last } \\
\text { time point }\end{array}$ & $\begin{array}{l}\text { Unable to adjust for } \\
\text { effects of clustering }\end{array}$ & $\begin{array}{l}\mathrm{n} / \mathrm{a} \text { - only two study } \\
\text { groups }\end{array}$ \\
\hline Ostelo 2003 & $\begin{array}{l}\text { Number of par- } \\
\text { ticipants withdrawn } \\
\text { from the study due } \\
\text { to worsening symp- } \\
\text { toms }\end{array}$ & Yes & $\begin{array}{l}\text { Numbers taken } \\
\text { from last time point }\end{array}$ & $\begin{array}{l}\mathrm{n} / \mathrm{a} \text { - not a cluster- } \\
\mathrm{RCT}\end{array}$ & $\begin{array}{l}\mathrm{n} / \mathrm{a} \text { - only two study } \\
\text { groups }\end{array}$ \\
\hline Parsons 2012 & $\begin{array}{l}\text { Number of par- } \\
\text { ticipants withdrawn } \\
\text { from the study due } \\
\text { to death }\end{array}$ & Yes & $\begin{array}{l}\mathrm{n} / \mathrm{a} \text { - only reported } \\
\text { on one measure at } \\
\text { one time point }\end{array}$ & $\begin{array}{l}\text { Unable to adjust for } \\
\text { effects of clustering }\end{array}$ & $\begin{array}{l}\mathrm{n} / \mathrm{a} \text { - only two study } \\
\text { groups }\end{array}$ \\
\hline
\end{tabular}

$\overline{\mathrm{CI}}=$ confidence interval; $\mathrm{n} / \mathrm{a}=$ not applicable; $\mathrm{RCT}=$ randomised controlled trial; $\mathrm{SD}=$ standard deviation 
Table 3. Outcome data from single studies

Comparison 1: Structured goal setting with or without strategies to enhance goal pursuit versus no goal setting

\section{Continuous data}

\begin{tabular}{|c|c|c|c|c|c|c|c|c|}
\hline \multirow[t]{2}{*}{ Trial } & \multirow{2}{*}{$\begin{array}{l}\text { Outcome } \\
\text { measure }\end{array}$} & \multicolumn{3}{|c|}{ Goal setting } & \multicolumn{3}{|c|}{ No goal setting } & \multirow[t]{2}{*}{ Mean difference $(95 \% \mathrm{CI})$} \\
\hline & & Mean & SD & $\mathbf{n}$ & Mean & SD & $\mathbf{n}$ & \\
\hline Bell 2003 & $\begin{array}{l}\text { Total Work } \\
\text { Behaviour } \\
\text { Inventory }\end{array}$ & 136.5 & 22.2 & 30 & 120.5 & 25.5 & 33 & 16.0 (4.22 to 27.78$)$ \\
\hline Bassett 1999 & $\begin{array}{l}\text { No. } \\
\text { treatment } \\
\text { sessions } \\
\text { required for } \\
\text { symptom re- } \\
\text { lief }\end{array}$ & 12.56 & 6.86 & 45 & 13.29 & 11.01 & 21 & $-0.73(-5.85$ to 4.39$)$ \\
\hline $\begin{array}{l}\text { Duncan } \\
2003\end{array}$ & $\begin{array}{l}\text { Baseline } \\
\text { Dyspnea In- } \\
\text { dex }\end{array}$ & 9.7 & 1.7 & 7 & 8.1 & 1.6 & 7 & $1.60(-0.13$ to 3.33$)$ \\
\hline $\begin{array}{l}\text { Duncan } \\
2003\end{array}$ & $\begin{array}{l}\text { Piper Fa- } \\
\text { tigue Scale }\end{array}$ & -1.8 & 1.7 & 7 & -2.2 & 1.9 & 7 & $0.40(-1.49$ to 2.29$)$ \\
\hline $\begin{array}{l}\text { Harwood } \\
2012\end{array}$ & $\begin{array}{l}\text { Systolic } \\
\text { blood pres- } \\
\text { sure } \\
(\mathrm{mmHg})\end{array}$ & 137.4 & 17.8 & 38 & 140.5 & 18.6 & 31 & $-3.50(-12.15$ to 5.15$)$ \\
\hline Mann 1987 & $\begin{array}{l}\text { Urinary } \\
\text { sodium out- } \\
\text { put (mmol/ } \\
24 \mathrm{~h})\end{array}$ & 157.4 & 52.7 & 19 & 141.4 & 57.9 & 19 & $16.0(-19.20$ to 51.20$)$ \\
\hline Mann 1987 & $\begin{array}{l}\text { Systolic } \\
\text { blood pres- } \\
\text { sure } \\
(\mathrm{mmHg})\end{array}$ & 135 & 17.8 & 19 & 137.6 & 17.2 & 19 & $-2.60(-13.73$ to 8.53$)$ \\
\hline Mann 1987 & $\begin{array}{l}\text { Criterion } \\
\text { referenced } \\
\text { achievement } \\
\text { test score }\end{array}$ & 18.8 & 2.7 & 19 & 17.1 & 3.3 & 19 & $1.70(-0.22$ to 3.62$)$ \\
\hline Sewell 2005 & $\begin{array}{l}\text { COPM sat- } \\
\text { isfaction }\end{array}$ & 2.04 & 1.91 & 63 & 2.27 & 2.03 & 58 & $-0.23(-0.93$ to 0.47$)$ \\
\hline
\end{tabular}


Table 3. Outcome data from single studies (Continued)

\begin{tabular}{|c|c|c|c|c|c|c|}
\hline \multicolumn{7}{|c|}{ Dichotomous data } \\
\hline \multirow[t]{2}{*}{ Trial } & \multirow[t]{2}{*}{ Outcome } & \multicolumn{2}{|c|}{ Goal setting } & \multicolumn{2}{|c|}{ No goal setting } & \multirow[t]{2}{*}{ Risk ratio $(95 \% \mathrm{CI})$} \\
\hline & & Events & Total & Events & Total & \\
\hline $\begin{array}{l}\text { Harwood } \\
2012\end{array}$ & $\begin{array}{l}\text { Depen- } \\
\text { dency, based } \\
\text { on modified } \\
\text { Rankin } \\
\text { Scores }>2\end{array}$ & 11 & 38 & 12 & 31 & $0.75(0.38$ to 1.46$)$ \\
\hline $\begin{array}{l}\text { Harwood } \\
2012\end{array}$ & Death & 4 & 46 & 5 & 39 & $0.68(0.20$ to 2.35$)$ \\
\hline $\begin{array}{l}\text { Howell } \\
1986\end{array}$ & $\begin{array}{l}\text { 'High' theo- } \\
\text { retical GAS } \\
\text { achievement }\end{array}$ & 8 & 13 & 7 & 11 & $0.97(0.52$ to 1.80$)$ \\
\hline $\begin{array}{l}\text { Iacovino } \\
1997\end{array}$ & $\begin{array}{l}\text { Return to } \\
\text { work }\end{array}$ & 15 & 22 & 21 & 24 & 0.78 (0.56 to 1.08$)$ \\
\hline
\end{tabular}

Comparison 2: Structured approach to goal setting with or without strategies to enhance goal pursuit versus 'usual care' that may have involved some goal setting but where no structured approach was followed

\section{Continuous data}

\begin{tabular}{|c|c|c|c|c|c|c|c|c|}
\hline \multirow[t]{2}{*}{ Trial } & \multirow{2}{*}{$\begin{array}{l}\text { Outcome } \\
\text { measure }\end{array}$} & \multicolumn{3}{|c|}{ Structured goal setting } & \multicolumn{3}{|c|}{ 'Usual care' goal setting } & \multirow[t]{2}{*}{ Mean difference $(95 \% \mathrm{CI})$} \\
\hline & & Mean & SD & $\mathbf{n}$ & Mean & SD & $\mathbf{n}$ & \\
\hline $\begin{array}{l}\text { Asenlof } \\
2005\end{array}$ & $\begin{array}{l}\text { Pain - Maxi- } \\
\text { mum }\end{array}$ & -3.6 & 3.17 & 28 & -4.9 & 3.1 & 37 & $1.30(-0.24$ to 2.84$)$ \\
\hline $\begin{array}{l}\text { Asenlof } \\
2005\end{array}$ & $\begin{array}{l}\text { Tampa Scale } \\
\text { of Kinesio- } \\
\text { phobia }\end{array}$ & -27.10 & 6.35 & 28 & -29.60 & 6.69 & 37 & $2.50(-0.69$ to 5.69$)$ \\
\hline Cheng 2012 & $\begin{array}{l}\text { Percent- } \\
\text { age of goals } \\
\text { achieved by } \\
\text { individual } \\
\text { participants }\end{array}$ & 98.0 & 9.3 & 40 & 61.5 & 49.0 & 29 & $36.5(18.43$ to 54.57$)$ \\
\hline $\begin{array}{l}\text { LaFerriere } \\
1978\end{array}$ & $\begin{array}{l}\text { Patient-re- } \\
\text { ported moti- } \\
\text { vation }\end{array}$ & 8.93 & 1.1 & 15 & 7.53 & 1.66 & 17 & $1.40(0.43$ to 2.37$)$ \\
\hline
\end{tabular}


Table 3. Outcome data from single studies (Continued)

\begin{tabular}{|c|c|c|c|c|c|c|c|c|}
\hline $\begin{array}{l}\text { LaFerriere } \\
1978\end{array}$ & $\begin{array}{l}\text { Therapist- } \\
\text { re- } \\
\text { ported moti- } \\
\text { vation of pa- } \\
\text { tients }\end{array}$ & 4.6 & 0.99 & 15 & 4.12 & 1.54 & 17 & $0.48(-0.41$ to 1.37$)$ \\
\hline $\begin{array}{l}\text { LaFerriere } \\
1978\end{array}$ & $\begin{array}{l}\text { Num- } \\
\text { ber of ther- } \\
\text { apy sessions } \\
\text { provided }\end{array}$ & 9.27 & 6.22 & 15 & 6.18 & 4.3 & 17 & $3.09(-0.66$ to 6.84$)$ \\
\hline Ostelo 2003 & $\begin{array}{l}\text { Change in } \\
\text { lumber } \\
\text { spine range } \\
\text { of } \\
\text { movement } \\
\text { (degrees) }\end{array}$ & 18.9 & 21.5 & 52 & 20.1 & 22.7 & 53 & $-1.20(-9.66$ to 7.26$)$ \\
\hline Ostelo 2003 & $\begin{array}{l}\text { Change in } \\
\text { Tampa Scale } \\
\text { of Kinesio- } \\
\text { phobia }\end{array}$ & 2.7 & 6.5 & 52 & 2.6 & 6.2 & 53 & $0.10(-2.33$ to 2.53$)$ \\
\hline Ostelo 2003 & $\begin{array}{l}\text { Total health- } \\
\text { care costs in } \\
\text { the } 12 \\
\text { month fol- } \\
\text { low-up pe- } \\
\text { riod (EUR) }\end{array}$ & 1978 & 1894 & 52 & 1339 & 1873 & 53 & $639(-81.61$ to 1359.61$)$ \\
\hline $\begin{array}{l}\text { Woltmann } \\
2011\end{array}$ & $\begin{array}{l}\text { Percent- } \\
\text { age of goals } \\
\text { recalled }\end{array}$ & 75 & 28 & 33 & 57 & 32 & 36 & $18.00(3.84$ to 32.16$)$ \\
\hline \multicolumn{9}{|c|}{ Dichotomous data } \\
\hline \multirow[t]{2}{*}{ Trial } & Outcome & \multicolumn{3}{|c|}{ Structured goal setting } & \multicolumn{3}{|c|}{ 'Usual care' goal setting } & Risk ratio $(95 \% \mathrm{CI})$ \\
\hline & & Events & Total & & Events & Total & & \\
\hline Arnetz 2004 & $\begin{array}{l}\text { Met or ex- } \\
\text { ceeded a } \\
\text { goal related } \\
\text { to range of } \\
\text { movement }\end{array}$ & 22 & 38 & & 8 & 32 & & $2.32(1.20$ to 4.47$)$ \\
\hline $\begin{array}{l}\text { Asenlof } \\
2005\end{array}$ & $\begin{array}{l}\text { More satis- } \\
\text { fied or much } \\
\text { more satis- }\end{array}$ & 33 & 38 & & 26 & 43 & & $1.27(0.94$ to 1.70$)$ \\
\hline
\end{tabular}


Table 3. Outcome data from single studies (Continued)

\begin{tabular}{|c|c|c|c|c|c|c|}
\hline & $\begin{array}{l}\text { fied with } \\
\text { daily living }\end{array}$ & & & & & \\
\hline $\begin{array}{l}\text { Asenlof } \\
2005\end{array}$ & $\begin{array}{l}\text { Healthcare } \\
\text { use over two } \\
\text { years (any } \\
\text { visits to doc- } \\
\text { tor, physio- } \\
\text { therapists, } \\
\text { or other care } \\
\text { givers due to } \\
\text { pain condi- } \\
\text { tions) }\end{array}$ & 19 & 28 & 19 & 37 & 1.32 (0.88 to 1.98$)$ \\
\hline $\begin{array}{l}\text { McPherson } \\
2009\end{array}$ & $\begin{array}{l}\text { Achieved or } \\
\text { exceeded at } \\
\text { least one set } \\
\text { goal }\end{array}$ & 7 & 13 & 7 & 9 & $0.69(0.38$ to 1.28$)$ \\
\hline
\end{tabular}

Comparison 4: One structured approach to goal setting and/or strategies to enhance goal pursuit versus another structured approach to goal setting and/or strategies to enhance goal pursuit

\section{Continuous data}

Collaborative versus prescribed (therapist mandated) goal setting

\begin{tabular}{|c|c|c|c|c|c|c|c|c|}
\hline \multirow[t]{2}{*}{ Trial } & \multirow{2}{*}{$\begin{array}{l}\text { Outcome } \\
\text { measure }\end{array}$} & \multicolumn{3}{|c|}{ Collaborative goal setting } & \multicolumn{3}{|c|}{ Prescribed goal setting } & \multirow[t]{2}{*}{ Mean difference $(95 \% \mathrm{CI})$} \\
\hline & & Mean & SD & $\mathbf{n}$ & Mean & SD & $\mathbf{n}$ & \\
\hline Bassett 1999 & $\begin{array}{l}\text { No. of treat- } \\
\text { ments } \\
\text { required }\end{array}$ & 13.61 & 8.01 & 23 & 11.46 & 5.4 & 22 & $2.15(-1.83$ to 6.13$)$ \\
\hline Bassett 1999 & $\begin{array}{l}\text { No. of home } \\
\text { exercise ses- } \\
\text { sions com- } \\
\text { plete }\end{array}$ & 75.43 & 20.88 & 23 & 65.82 & 23.65 & 22 & $9.61(-3.45$ to 22.67$)$ \\
\hline
\end{tabular}

Goal setting with versus without operant conditioning

\begin{tabular}{|c|c|c|c|c|c|c|c|c|}
\hline \multirow[t]{2}{*}{ Trial } & \multirow[t]{2}{*}{$\begin{array}{l}\text { Outcome } \\
\text { measure }\end{array}$} & \multicolumn{3}{|c|}{$\begin{array}{l}\text { Goal setting plus operant } \\
\text { conditioning }\end{array}$} & \multicolumn{3}{|c|}{$\begin{array}{l}\text { Goal setting without operant } \\
\text { conditioning }\end{array}$} & Mean difference $(95 \% \mathrm{CI})$ \\
\hline & & Mean & SD & $\mathbf{n}$ & Mean & SD & $\mathbf{n}$ & \\
\hline
\end{tabular}


Table 3. Outcome data from single studies (Continued)

\begin{tabular}{|c|c|c|c|c|c|c|c|c|}
\hline Blair 1991 & $\begin{array}{l}\text { Rosen- } \\
\text { berg Self-Es- } \\
\text { teem Scale }\end{array}$ & 1.185 & 1.111 & 27 & 1.577 & 0.703 & 26 & $-0.39(-0.89$ to 0.11$)$ \\
\hline \multicolumn{9}{|c|}{ End-only goals versus end goals with short-term steps } \\
\hline \multirow[t]{2}{*}{ Trial } & \multirow[t]{2}{*}{$\begin{array}{l}\text { Outcome } \\
\text { measure }\end{array}$} & \multicolumn{3}{|c|}{ End-only goal } & \multicolumn{3}{|c|}{$\begin{array}{l}\text { End goal with short-term } \\
\text { steps }\end{array}$} & Mean difference $(95 \% \mathrm{CI})$ \\
\hline & & Mean & SD & $\mathbf{n}$ & Mean & SD & $\mathbf{n}$ & \\
\hline $\begin{array}{l}\text { Conrad } \\
2000\end{array}$ & $\begin{array}{l}\text { Self- } \\
\text { reported di- } \\
\text { etary fat } \\
\text { con- } \\
\text { sumption as } \\
\text { a percentage } \\
\text { of total en- } \\
\text { ergy con- } \\
\text { sumption }\end{array}$ & 34 & 6 & 4 & 27 & 15.6 & 3 & $7.00(-11.61$ to 25.61$)$ \\
\hline
\end{tabular}

Specific versus a non-specific goal

\begin{tabular}{l|lll|llllll}
\hline Trial & $\begin{array}{l}\text { Outcome } \\
\text { measure }\end{array}$ & Specific goals & & Non-specific goals & & Mean difference (95\% CI) \\
\hline & Mean & SD & n & Mean & SD & n & \\
\hline James 1993 & $\begin{array}{l}\text { Pain } \\
\text { behaviour } \\
\text { nonverbal } \\
\text { complaint }\end{array}$ & 1.28 & 1.34 & 13 & 4.3 & 5 & 13 & $-3.02(-5.83$ to -0.21$)$ \\
\hline
\end{tabular}

Difficult versus easier goals

\begin{tabular}{|c|c|c|c|c|c|c|c|c|}
\hline \multirow[t]{2}{*}{ Trial } & \multirow{2}{*}{$\begin{array}{l}\text { Outcome } \\
\text { measure }\end{array}$} & \multicolumn{3}{|c|}{ Difficult goal (8 servings) } & \multicolumn{3}{|c|}{ Easy goal (6 servings) } & \multirow[t]{2}{*}{ Mean difference $(95 \% \mathrm{CI})$} \\
\hline & & Mean & SD & $\mathbf{n}$ & Mean & SD & $\mathbf{n}$ & \\
\hline Miller 2012 & $\begin{array}{l}\text { Total num- } \\
\text { ber of serv- } \\
\text { ings lower } \\
\text { glycaemic } \\
\text { index foods } \\
\text { per day }\end{array}$ & 8.4 & 0.83 & 20 & 8.42 & 0.96 & 15 & $-0.02(-0.63$ to 0.59$)$ \\
\hline Miller 2012 & $\begin{array}{l}\text { Goal com- } \\
\text { mitment }\end{array}$ & 4.09 & 0.12 & 20 & 4.5 & 0.14 & 15 & $-0.41(-0.50$ to -0.32$)$ \\
\hline
\end{tabular}


Table 3. Outcome data from single studies (Continued)

\begin{tabular}{|c|c|c|c|c|c|c|c|c|}
\hline Miller 2012 & $\begin{array}{l}\text { Satis- } \\
\text { faction with } \\
\text { goal achieve- } \\
\text { ment }\end{array}$ & 6.8 & 0.36 & 20 & 6.47 & 0.41 & 15 & $0.33(0.07$ to 0.59$)$ \\
\hline Miller 2012 & $\begin{array}{l}\text { Self-efficacy } \\
\text { - total score }\end{array}$ & 9.3 & 0.28 & 20 & 9.33 & 0.32 & 15 & $-0.03(-0.23$ to 0.17$)$ \\
\hline
\end{tabular}

High intensity exercise goals versus non-specific intensity exercise goals

\begin{tabular}{|c|c|c|c|c|c|c|c|c|}
\hline \multirow[t]{2}{*}{ Trial } & \multirow[t]{2}{*}{$\begin{array}{l}\text { Outcome } \\
\text { measure }\end{array}$} & \multicolumn{3}{|c|}{$\begin{array}{l}\text { High intensity exercise } \\
\text { goal }\end{array}$} & \multicolumn{3}{|c|}{$\begin{array}{l}\text { Non-specific exercise inten- } \\
\text { sity goal }\end{array}$} & \multirow[t]{2}{*}{ Mean difference $(95 \% \mathrm{CI})$} \\
\hline & & Mean & SD & $\mathbf{n}$ & Mean & SD & $\mathbf{n}$ & \\
\hline $\begin{array}{l}\text { Richardson } \\
2007\end{array}$ & $\begin{array}{l}\text { Total } \\
\text { steps during } \\
\text { any walking }\end{array}$ & 6868 & 3751 & 13 & 6279 & 3306 & 17 & $589(-1985.37$ to 3163.37$)$ \\
\hline $\begin{array}{l}\text { Richardson } \\
2007\end{array}$ & $\begin{array}{l}\text { Steps counts } \\
\text { dur- } \\
\text { ing bouts of } \\
\text { high inten- } \\
\text { sity walking }\end{array}$ & 2616 & 2706 & 13 & 2070 & 2814 & 17 & $546(-1442.24$ to 2534.24$)$ \\
\hline $\begin{array}{l}\text { Richardson } \\
2007\end{array}$ & $\begin{array}{l}\text { Duration } \\
\text { pedometers } \\
\text { worn per } \\
\text { day (hours) }\end{array}$ & 14.5 & 2.49 & 13 & 16.5 & 2.49 & 17 & $-2.00(-3.80$ to -0.20$)$ \\
\hline \multicolumn{9}{|c|}{ Dichotomous data } \\
\hline \multicolumn{9}{|c|}{ Goal Management Training versus Identity Oriented Goal Mapping } \\
\hline \multirow[t]{2}{*}{ Trial } & Outcome & \multicolumn{3}{|c|}{$\begin{array}{l}\text { Goal Management Train- } \\
\text { ing }\end{array}$} & \multicolumn{3}{|c|}{$\begin{array}{l}\text { Identity Orientated Goal } \\
\text { Mapping }\end{array}$} & Risk ratio $(95 \% \mathrm{CI})$ \\
\hline & & Events & Total & & Events & Total & & \\
\hline $\begin{array}{l}\text { McPherson } \\
2009\end{array}$ & $\begin{array}{l}\text { Achieved or } \\
\text { exceeded at } \\
\text { least one set } \\
\text { goal }\end{array}$ & 3 & 8 & & 4 & 5 & & $0.47(0.17$ to 1.27$)$ \\
\hline
\end{tabular}

High intensity exercise goals versus non-specific intensity exercise goals

\begin{tabular}{l|l|l}
\hline Trial Outcome & $\begin{array}{l}\text { High intensity exercise } \\
\text { goal }\end{array}$ & $\begin{array}{l}\text { Non-specific exercise inten- Risk ratio (95\% CI) } \\
\text { sity goal }\end{array}$
\end{tabular}


Table 3. Outcome data from single studies (Continued)

\begin{tabular}{|c|c|c|c|c|c|c|}
\hline & & Events & Total & Events & Total & \\
\hline $\begin{array}{l}\text { Richardson } \\
2007\end{array}$ & $\begin{array}{l}\text { Would defi- } \\
\text { nitely } \\
\text { recommend } \\
\text { the pro- } \\
\text { gramme to a } \\
\text { friend }\end{array}$ & 8 & 13 & 17 & 17 & $0.62(0.41$ to 0.96$)$ \\
\hline $\begin{array}{l}\text { Richardson } \\
2007\end{array}$ & $\begin{array}{l}\text { Programme } \\
\text { considered } \\
\text { very useful }\end{array}$ & 4 & 13 & 12 & 17 & $0.44(0.18$ to 1.04$)$ \\
\hline
\end{tabular}

$\overline{\mathrm{COPM}}$ = Canadian Occupational Performance Measure; GAS = Goal Attainment Scaling

\section{A P P E N D I C ES}

\section{Appendix I. CENTRAL search strategy}

\#1 MeSH descriptor Rehabilitation explode all trees

\#2 (rehab* or recover*):ti,ab,kw,so

\#3 MeSH descriptor Rehabilitation Centers explode all trees

\#4 MeSH descriptor Physical Therapy Modalities explode all trees

\#5 convalescence:kw

\#6 (physiotherap* or physical-therap* or occupational-therap* or neurorehabilitation or nurs*):ti,ab,kw

$\# 7$ *therap*:ti,kw,so

\#8 (\#1 OR \#2 OR \#3 OR \#4 OR \#5 OR \#6 OR \#7)

\#9 (goal near/5 (set* or plan* or agree* or negotiat* or discuss* or propos* or prescrib* or develop* or formulat* or elaborat* or establish*

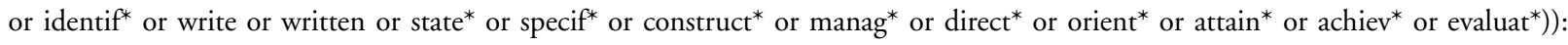
ti,ab,kw

\#10 (target* near/1 behavio*):ti,ab,kw

\#11 (set* near/2 target):ti,ab,kw

\#12 (\#9 OR \#10 OR \#11)

\#13 (\#8 AND \#12)

\#14 (goal near/1 (set* or plan* or attain* or directed or orient* or cent* ${ }^{*}$ red)):ti,ab,kw

\#15 (\#13 OR \#14) 


\section{Appendix 2. MEDLINE (Ovid SP) search strategy}

1. exp rehabilitation/

2. rehabilitation.mp.

3. (rehab* or recover*).tw.

4. rh.fs.

5. exp rehabilitation centers/

6. recovery of function/

7. exp physical therapy modalities/

8. (physiotherap* or physical therap* or occupational therap* or neurorehabilitation or nurs*).tw.

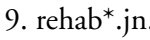

10. or/1-9

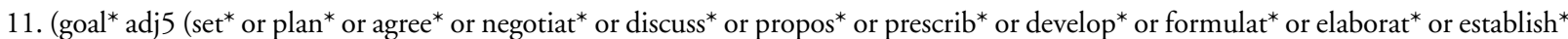
or identif* or write or written or state* or specif* or construct* or manag* or direct* or orient* or attain* or achiev* or evaluat*)).tw.

12. (target* adj1 behavio*).tw.

13. $\left(\right.$ set* $^{*}$ adj2 target $\left.^{*}\right)$.tw.

14. or/11-13

15. 10 and 14

16. (goal adj1 (set* or plan* or attain* or directed or oriented or cent?red)).tw.

17. goals/

18. 16 or 17

19. 15 or 18

20. randomized controlled trial.pt.

21. controlled clinical trial.pt.

22. randomized.ab.

23. placebo.ab.

24. drug therapy.fs.

25 . randomly.ab.

26. trial.ab.

27. groups.ab.

28. or/20-27

29. exp animals/ not humans.sh.

30. 28 not 29

31.19 and 30

\section{Appendix 3. EMBASE (OvidSP) search strategy}

1. exp rehabilitation/

2. rehabilitation.hw.

3. (rehab* or recover*).ti,ab,kw.

4. rh.fs.

5. convalescence/

6. exp physiotherapy/

7. (physiotherap* or physical therap* or occupational therap* or neurorehabilitation or nurs*).ti,ab,kw.

8. rehab*.jn.

9. or/ $1-8$

10. (goal* adj5 (set* or plan* or agree* or negotiat* or discuss* or propos* or prescrib* or develop* or formulat* or elaborat* or establish*

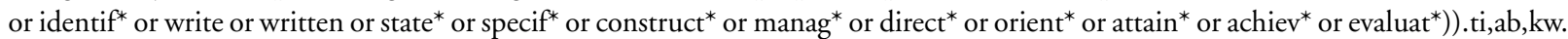

11. (target* adj1 behavio*).ti,ab,kw.

12. (set* $\left.\operatorname{adj} 2 \operatorname{target}{ }^{*}\right) \cdot \mathrm{ti}, \mathrm{ab}, \mathrm{kw}$.

13. or $/ 10-12$

14.9 and 13

15. (goal adj1 (set* or plan* or attain* or directed or orient* or cent?red)).ti,ab,kw.

Goal setting and strategies to enhance goal pursuit for adults with acquired disability participating in rehabilitation (Review)

Copyright $\odot 2015$ The Cochrane Collaboration. Published by John Wiley \& Sons, Ltd. 
16. 14 or 15

17. randomized controlled trial/

18. controlled clinical trial/

19. single blind procedure/ or double blind procedure/

20. crossover procedure/

21. random*.tw.

22. placebo*.tw. $^{*}$

23. ((singl* or doubl*) adj (blind* or mask*)).tw.

24. (crossover or cross over or factorial* or latin square).tw.

25. (assign* or allocat* or volunteer*).tw.

26. or/17-25

27. nonhuman/ not (human/ and nonhuman/)

28. 26 not 27

29. 16 and 28

\section{Appendix 4. PsycINFO (OvidSP) search strategy}

1. exp rehabilitation/

2. rehabilitation.hw.

3. (rehab* or recover $\left.^{*}\right)$.ti,ab,id.

4. exp rehabilitation centers/

5. "recovery (disorders)"/

6. (physiotherap* or physical therap* or occupational therap* or neurorehabilitation or nurs*).ti,ab,id.

7. rehab*.jn.

8. or/ $1-7$

9. (goal* adj5 (set* or plan* or agree* or negotiat* or discuss* or propos* or prescrib* or develop* or formulat* or elaborat* or establish* or identif* or write or written or state* or specif* or construct* or manag* or direct* or orient* or attain* or achiev* or evaluat*)).ti,ab,id.

10. (target* adj1 behavio*).ti,ab,id.

11. (set* adj$^{2}$ target*).ti,ab,id.

12. or $/ 9-11$

13. 8 and 12

14. (goal adj1 (set* or plan* or attain* or directed or orient* or cent?red)).ti,ab,id.

15. goal setting/

16. goal orientation/

17. or/14-16

18. 13 or 17

19. random*.ti,ab,hw,id.

20. trial*.ti,ab,hw,id.

21. controlled study.ti,ab,id.

22. placebo*.ti,ab,hw,id.

23. ((singl* ${ }^{*}$ or doubl* or trebl* or tripl*) and (blind* or mask $\left.\left.{ }^{*}\right)\right)$.ti,ab,hw,id.

24. (cross over or crossover or factorial* or latin square).ti,ab,hw,id.

25. (assign* or allocat* or volunteer*).ti,ab,hw,id.

26. treatment effectiveness evaluation/

27. mental health program evaluation/

28. exp experimental design/

29. " 2000 ”. md.

30. or/19-29

31. 18 and 30

Goal setting and strategies to enhance goal pursuit for adults with acquired disability participating in rehabilitation (Review) 


\section{Appendix 5. CINAHL (EBSCOhost) search strategy}

S1 MH Rehabilitation+

S2 TX rehabilitation

S3 TI (rehab* or recover*) or AB (rehab* or recover*)

S4 MH Recovery

S5 MH Rehabilitation Centers+

S6 TI (physiotherap* or physical therap* or occupational therap* or neurorehabilitation or nurs*) or AB (physiotherap* or physical therap* or occupational therap* or neurorehabilitation or nurs*)

S7 TI target* N2 (behavio* or set*) or AB target* N2 (behavio* or set*)

S8 TI goal* N4 (set* or plan* or agree* or negotiat* or discuss* or propos* or prescrib* or develop* or formulat* or elaborat* or establish*

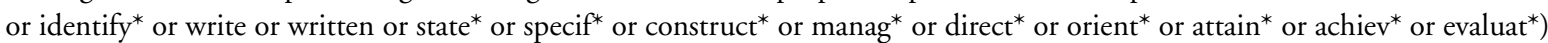

S9 AB goal* N4 (set* or plan* or agree* or negotiat* or discuss* or propos* or prescrib* or develop* ${ }^{*}$ or formulat* or elaborat* ${ }^{*}$ or establish* or identify* or write or written or state* or specif* or construct* or manag* or direct* or orient* or attain* or achiev* or evaluat*)

$\mathrm{S} 10 \mathrm{~S} 1$ or $\mathrm{S} 2$ or $\mathrm{S} 3$ or $\mathrm{S} 4$ or $\mathrm{S} 5$ or $\mathrm{S} 6$

S11 S7 or S8 or S9

S12 S10 and S11

S13 TI goal N1 (set* or plan* or attain* or directed or oriented or cent*red)

S14 AB goal N1 (set* or plan* or attain* or directed or oriented or cent*red)

S15 MH goals and objectives

S16 MH goal-setting

S17 MH goal attainment

$S 18 S 13$ or $S 14$ or $S 15$ or $S 16$ or $S 17$

S19 S18 or S12

S20 PT randomized controlled trial

S21 PT Clinical Trial

S22 MH Clinical Trials+

S23 MH Random

S24 MH Placebos

S25 MH Quantitative Studies

S26 AB (random* or trial or placebo*) or TI (random* or trial or placebo*)

S27 AB (singl* or doubl* or tripl* or trebl*) and AB (blind* or mask*)

S28 TI (singl* or doubl* or tripl* or trebl*) and TI (blind* or mask*)

S29 S20 or S21 or S22 or S23 or S24 or S25 or S26 or S27 or S28

S30 S29 and S19

S31 S30 Limiters - Exclude MEDLINE records

\section{Appendix 6. AMED (OvidSP) search strategy}

1. exp Rehabilitation/

2. rehabilitation.mp.

3. (rehab* or recover*).tw.

4. exp Rehabilitation centers/

5. exp physical therapy modalities/

6. (physiotherap* or physical therap* or occupational therap* or neurorehabilitation or nurs*).tw.

7. rehab*.jn.

8. or/ $1-7$

9. (goal* adj5 (set* or plan* or agree* or negotiat* or discuss* or propos* or prescrib* or develop* ${ }^{*}$ or formulat* or elaborat* or establish*

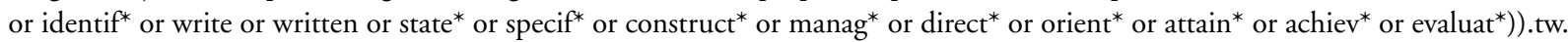

10. (target* adj1 behavio*).tw.

11. (set* adj2 target*).tw.

12. or/9-11

Goal setting and strategies to enhance goal pursuit for adults with acquired disability participating in rehabilitation (Review)

Copyright @ 2015 The Cochrane Collaboration. Published by John Wiley \& Sons, Ltd. 
13. 8 and 12

14. (goal adj1 (set* or plan* or attain* or directed or oriented or cent?red)).tw.

15. goals/

16. 14 or 15

17. 13 or 16

18. randomized controlled trial.pt.

19. controlled clinical trial.pt.

20. (random* or trial or placebo* or single blind* or double blind* or crossover or cross over or factorial or latin square or assign* ${ }^{*}$ or allocat* or volunteer*).mp.

21. or/ $18-20$

22. 17 and 21

\section{Appendix 7. Proquest Dissertations and Theses database search strategy}

(Command Line Search)

all((goal* N/5 (set* OR plan* OR agree* OR negotiat* OR discuss* OR propos* OR prescrib* OR develop* OR formulat* OR elaborat* OR establish* OR identif* OR write OR written OR state* OR specif* OR construct* OR manag* OR direct* OR orient* OR attain* OR achiev* OR evaluat*)) AND (rehab* OR recover* OR neurorehab* OR therap* physiotherap* OR nurs*) AND (random* OR trial OR “controlled study" OR placebo* OR assign* OR allocat* OR “double blind*” OR "single blind"” OR crossover OR "cross over" OR factorial OR "latin square"))

\section{Appendix 8. Searches of clinical trial registries}

WHO Clinical Trial Search Portal (www.who.int/trialsearch)

(Command Line Search)

"goal setting" OR "goal planning" OR "goal orient*"

Australian New Zealand Clinical Trials Registry (http://www.anzctr.org.au/)

Three searches using the following terms: "goal setting", "goal planning", "goal orient*"

Current Controlled Trials (www.controlled-trials.com)

Three searches using the following terms: "goal setting", "goal planning", "goal orient*"

\section{CONTRIBUTIONSOFAUTHORS}

All authors contributed to the conceptualisation of the review. WL prepared the first draft of the protocol, with input from all other authors during the further development of this protocol. WL undertook all library database searches and all communication with study authors, developed and maintained the databases which were used to extract and manage study data, and prepared the first draft of the completed review and all revision of the review following peer review. WL and RS conducted all screening of potential titles and abstracts, the independent critical appraisal of studies, and all data extraction. WL entered the data into Review Manager 5, which was checked for accuracy by RS. MW advised on biostatistical issues and undertook the advanced biostatistical analyses, such as adjustment of CIs to account for clusters-RCT designs and the individual subject meta-analysis for the Comparison 3 studies, which could not be conducted within Review Manager 5. JHS provided additional methodological advice on the Cochrane review process, including the interpretation of research methods and pooling for outcome data for the purpose of a Cochrane review. All authors provided content expertise and contributed to the final version of the review for publication. 


\section{DECLARATIONSOF INTEREST}

SD's position at the University of Exeter Medical School is supported by the National Institute for Health Research (NIHR) Collaboration for Leadership in Applied Health Research and Care South West Peninsula at the Royal Devon and Exeter NHS Foundation Trust. The views expressed are those of the author(s) and not necessarily those of the National Health Service (NHS), the NIHR or the Department of Health.

RS and WL are co-editors of Siegert $2014 \mathrm{~b}$ for which they may receive royalties.

WL has in the past received some financial gifts from health professional organisations in the UK for presenting on the topic of goal setting in rehabilitation (Association of Chartered Physiotherapists Interested in Neurology (2013); University College London (2015)).

KM's funded research (The Health Research Council 2005-2011) includes a focus on goal setting. AUT University has received payment from the Accident Compensation Corporation (New Zealand) (2010-2015) for consultancy work advising the Corporation on goals and goal setting.

WL, JHS, and MW are employed as academics by the University of Otago. RS is employed as an academic by AUT University. KM is employed as Chief Executive by the Health Research Council of New Zealand.

\section{SOURCES OF SUPPORT}

\section{Internal sources}

- Rehabilitation Teaching and Research Unit, Department of Medicine, University of Otago (Wellington), New Zealand.

WL, JHS, and MW all hold posts in the Department of Medicine, funded by the University of Otago

- National Institute of Health Research, UK.

SD's position at the University of Exeter Medical School is supported by the National Institute for Health Research (NIHR)

Collaboration for Leadership in Applied Health Research and Care South West Peninsula at the Royal Devon and Exeter NHS

Foundation Trust. The views expressed are those of the author(s) and not necessarily those of the NHS, the NIHR or the Department of Health.

- Laura Fergusson Trust (Auckland) and AUT University, New Zealand.

KM's Chair in Rehabilitation is part funded by the Laura Fergusson Trust (Auckland) and part funded by AUT University RS holds a post in the School of Public Health and Psychosocial Studies and School of Rehabilitation and Occupation Studies, funded by AUT University

\section{External sources}

- No sources of support supplied

\section{DIFFERENCES BETWEEN PROTOCOLANDREVIEW}

Categorisation and analysis of studies by comparison type: In our protocol we stated that our objective was to examine three types of comparisons in clinical trials on rehabilitation goal setting and strategies to enhance goal pursuit.

1. Goal setting interventions versus no goal setting.

2. Interventions to enhance goal pursuit versus no interventions to enhance goal pursuit.

3. One approach to goal setting and/or strategies to enhance goal pursuit versus another approach to goal setting and/or strategies to enhance goal pursuit.

However, on examination of the papers and theses that emerged from our literature search, we identified a fourth group of studies where one structured approach to goal setting and/or strategies to enhance goal pursuit had been compared to 'usual practice' that may include some goals being set, but not necessarily for all patients and not following any structured or required approach to goal setting

Goal setting and strategies to enhance goal pursuit for adults with acquired disability participating in rehabilitation (Review) 
(i.e. where healthcare professionals were permitted to set whatever goals they thought most appropriate, or not to set goals, without any commitment to a specific goal setting strategy). Therefore our revised comparisons, as stated in the Objectives, were as follows.

1. A structured approach to goal setting with or without strategies to enhance goal pursuit versus no goal setting.

2. A structured approach to goal setting with or without strategies to enhance goal pursuit versus 'usual care' that may involve some goal setting but where no structured approach was followed.

3. Interventions to enhance goal pursuit versus no interventions to enhance goal pursuit.

4. One structured approach to goal setting and/or strategies to enhance goal pursuit versus another structured approach to goal setting and/or strategies to enhance goal pursuit.

Databases included in the search strategy: We originally included searches of Sociological Abstracts and The Cochrane Consumers and Communication Review Group Specialised Register in our Electronic searches, but access to Sociological Abstracts was not available and The Cochrane Consumers and Communication Review Group Specialised Register was no longer in use, so these searches were omitted from the review.

Unit of analysis issues related to repeat observations: In the protocol we stated that we would deal with unit of analysis issues by following recommendations in the Cochrane Handbook for Systematic Review of Interventions (Higgins 2011). In the review we clarified that for studies with repeat observations (collecting data using the same measures on participants at a number of different time points) this meant that we selected the longest follow-up data from each study for meta-analysis of effect sizes.

Unit of analysis issues related to cluster-RCTs: For three cluster-RCT in this review, it was not possible to follow any of the initially planned strategies for adjusting results to account for the effects of clustering. Insufficient data were reported in these studies to account for clustering, the authors of the studies were unable to be contacted or unable to provide the type of raw data required to estimate variance arising from clustering, and no relevant estimates of variance could be identified in any other published studies using the same measures in similar contexts. We therefore chose to include data from these cluster-RCTs in the review without adjusting for clustering, but to explicitly report when we did this, and to test the effect of including these studies by also examining and reporting the results of the analyses with these studies removed.

Analysis of data: Following data extraction, but prior to data analysis, we made the post-hoc decision to combine measures of selfreported emotional status with measures of health-related quality of life. We did this because insufficient studies reported measures of health-related quality of life, and because the two concepts were deemed to be sufficiently similar for the results in a meta-analysis to be clinically meaningful. 